Portland State University

PDXScholar

Spring 5-1-2014

\title{
'This Would Be Much Funner in Person': A Qualitative Grounded Theory Analysis of Cybergrooming
}

Maurice Jason Gauz

Portland State University

Follow this and additional works at: https://pdxscholar.library.pdx.edu/open_access_etds

Part of the Discourse and Text Linguistics Commons, and the Social Control, Law, Crime, and Deviance Commons

Let us know how access to this document benefits you.

\section{Recommended Citation}

Gauz, Maurice Jason, "'This Would Be Much Funner in Person': A Qualitative Grounded Theory Analysis of Cybergrooming" (2014). Dissertations and Theses. Paper 1802.

https://doi.org/10.15760/etd.1802

This Thesis is brought to you for free and open access. It has been accepted for inclusion in Dissertations and Theses by an authorized administrator of PDXScholar. Please contact us if we can make this document more accessible: pdxscholar@pdx.edu. 
'This Would Be Much Funner in Person'

A Qualitative Grounded Theory Analysis of Cybergrooming

by

Maurice Jason Gauz

A thesis submitted in partial fulfillment of the requirements for the degree of

Master of Science

in

Communication

Thesis Committee:

Jeffrey Robinson, Chair

Priya Kapoor

Randy Blazak

Portland State University 2014 
(C) 2014 Maurice Jason Gauz 


\begin{abstract}
The rather novel phenomenon of cybergrooming, or the solicitation of minors for sex via the Internet, has yet to be fully explored. This is a problem because such predatory behavior can lead to psychological and/or physical abuse of minors. The present study seeks to fill this knowledge by performing a qualitative, grounded theory analysis of naturally-occurring cybergrooming discourse. Data were drawn from the website of the online watchdog group, Perverted Justice. The first 20 lines of talk transmitted by the adults in these chat conversations were sampled from 100 transcripts published by Perverted Justice.

Multi-step coding, facilitated by the qualitative analysis software Atlas.ti, revealed 11 themes of social action that discursively emerged in at least $25 \%$ of said transcripts: (1) conversational openings; (2) initial solicitation of age, sex and/or location; (3) specific questions regarding 'child's' life; (4) follow-up topicalization of 'child's' location; (5) seeking visual images of 'child;' (6) complimenting 'child's' appearance; (7) soliciting topic for discussion; (8) explicitly sexual statements; (9) soliciting 'child's' age preference for sex/romance; (10) arranging further contact; and (11) disclosing personal activities. These themes are then explored in their own context, in relation to each other, and as elements of the broad behavioral framework of cybergrooming.
\end{abstract}


Table of Contents

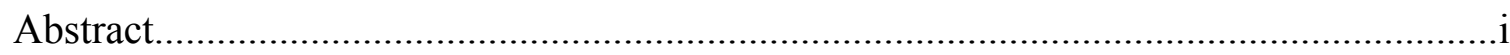

Chapter 1

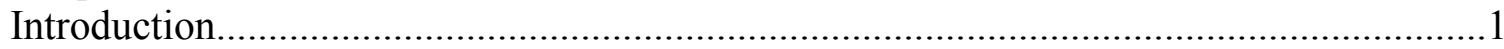

Chapter 2

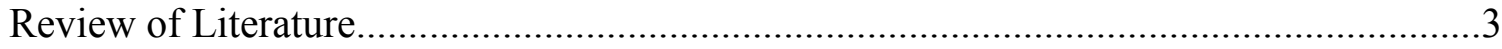

Chapter 3

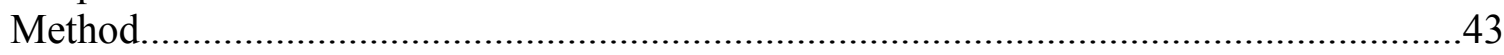

Chapter 4

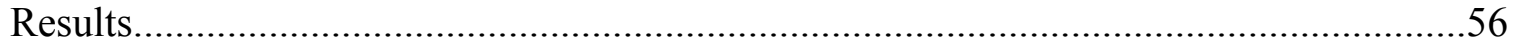

Chapter 5

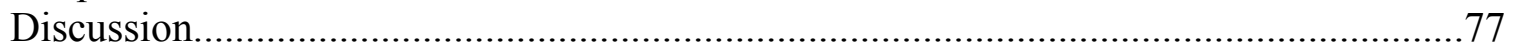

Chapter 6

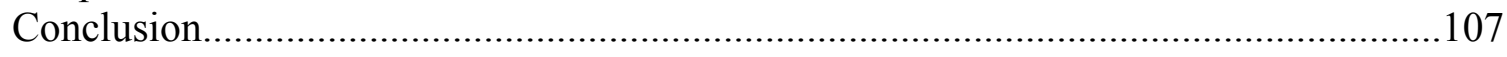

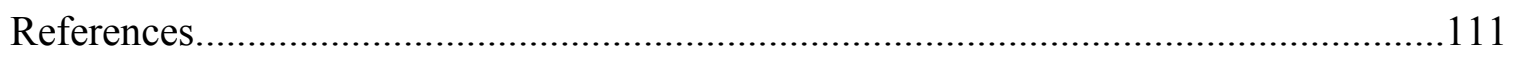

Appendix

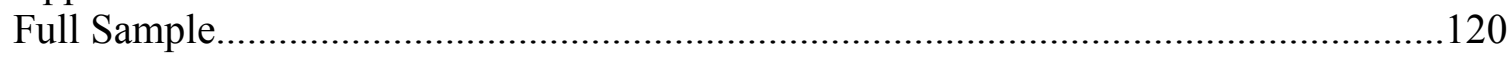




\section{Chapter 1: Introduction}

The following study is an in-depth qualitative analysis of online chat transcripts during which an adult is attempting to solicit a minor for sex, otherwise known as cybergrooming. ${ }^{1}$ This type of research is needed because young people are regularly propositioned in this manner (Berson, 2003; Marcum, 2007), and related work has shown that early overexposure to sexual ideas and discussion — even without physical abusecan be psychologically harmful to youth (Longo \& Groth, 1983). Both the topic area and approach I adopted for this project are relatively novel ideas; very little research has been conducted into the phenomenon of child predation via the Internet. The few studies that have emerged tend to take a broad angle, applying traditional facets of sex crime research to related online behavior. This deductive methodology has left a gap in the literature as no known work has inductively analyzed online sexual predation against minors from a grounded approach that prioritizes utterance-level analysis of naturally-occurring synchronous chat of this type.

The present study begins to fill this gap by focusing in on naturally-occurring cybergrooming communication, prioritizing members' meanings, and scrutinizing specific utterances within their localized contexts in order to explore how they achieve social actions and what themes discursively emerge. I was specifically interested in how the adults in these interactions conversationally initiated cybergrooming, and thus my research process focused on the adults' statements within the opening sequences of confirmed cybergrooming attempts. I investigated a large sample of data of this type,

\footnotetext{
${ }^{1}$ Cybergrooming can be defined as "establishing a trust-based relationship between minors and usually adults using [computer-mediated communication] to systematically solicit and exploit the minors for sexual purposes" (Wachs, Wolf \& Pan, 2012, p. 628). See 'Cybergrooming' subsection for detailed explication.
} 

social actions performed with widely varied patterns of sequencing and organization, which are then presented as thematic elements of cybergrooming behavior. ${ }^{3}$ The subsequent discussion explicates these themes as individual but intertwined facets of this phenomenon and explores how the findings further existing knowledge reported in prior studies, which offer many useful—but often incomplete or misleadingly rigid frameworks - for this discursive process. The present study thus provides a useful guide to identifying potentially predatory online behavior as it manifests, which is a necessary and valuable preventative tool for education, law enforcement, and public safety.

\footnotetext{
2 for description of this organization, its tactics, and the resulting data set, see Chapter III: Methods. ${ }^{3}$ See Chapter IV: Results.
} 


\section{Chapter 2: Review of Literature}

The following literature review is a comprehensive synthesis of prior research conducted on cybergrooming as well as related topics and salient communication theory, exposing where knowledge is missing and proving the need for inductive analysis of this phenomenon. I begin by explicating how the dynamics and communicative features that characterize the Internet can facilitate or otherwise affect child sexual predation. The next subsection addresses the tradition of (offline) sexual luring and grooming literature, with a focus on communicative concepts. Following this is a detailed review of emerging cybergrooming literature, which reveals a dearth of truly inductive approaches. The final section outlines the Conversation Analytic framework which conceptually informed my analysis. The chapter concludes with the research questions that emerged from the literature and subsequently guided the planning and execution of this study.

\section{The Internet and Sexual Predation}

Technological advancements and new media almost always affect the communication and behavior of their users, and this is the case also for sexual deviants (Quinn \& Forsyth, 2005). While the Internet allows for a certain level of anonymity, it often also encourages individuals to discard their privacy by publicly displaying personally identifiable information (Berson, 2003; Wolak, Finkelhor, Mitchell, \& Ybarra, 2008). Both of these features are salient to the topic of online sexual predation. Therefore, this section reviews, in detail, common sexually deviant uses of the medium, characteristics of the Internet and chat rooms that facilitate sexual predation, and finally, statistics associated with youth Internet/chat room use and who may be most at-risk for victimization. 
Online deviance. Quinn and Forsyth (2005) state that the Internet has been found to act as a substitute for sexual behaviors normally deemed abnormal and "represents an important means of sexual expression for an increasing number of individuals that is not immediately accessible to societal constraints" (p. 197). Such activities can include prostitution, sex trafficking, child pornography, and a host of other activities associated with a deviant script. The Internet not only facilitates access to information about these behaviors but also allows people to seek out and communicate with, via chat rooms, forums, and online communities, others who have similar desires (Holt, Blevins, \& Burkert, 2010; Quayle \& Taylor, 2003; Quinn \& Forsyth, 2005; Webster et al., 2010). One other such behavior-sexual communication with, and exploitation of - minors, is the focus of the present study.

Marcum (2007) delineated four ways in which adults who are sexually interested in minors utilize the Web: child pornography exchange, communicating with other pedophiles, locating victims, and inappropriately engaging young people in sexualized conversations. This final practice is most relevant to the present study, and the author further points out that some alleged abusers do not attempt to meet their victim(s) for physical sex and achieve gratification from the knowledge that they are actively engaging youth in sexually charged discourse. These individuals maintain an online-only relationship with the child, often for months at a time (Wolak, Finkelhor, \& Mitchell, 2004), and claim this prevents actual harm to the child. However, most Western nations now consider online-only sexual communication between adults and minors (often termed online grooming or cybergrooming) a crime of sexual predation (Jewkes, 2010; Shannon, 2008). Additionally, Marcum (2007) points out that while "the Internet can 
facilitate the communication of sexual desires through words, the heated conversations often lead to requests for face-to-face meetings that result in sexual activity" (p. 103). Therefore, the remainder of this subsection will be dedicated to reviewing Internet and chat room features that make them ideal sites for targeting children for sexual exploitation, followed by relevant statistics on youth Internet/chat room communication and their vulnerability to victimization.

Internet features that facilitate predation. Despite the many benefits the Internet has to offer society, its dark side - particularly its capacity to facilitate harm to children - is no secret. Davidson and Gottschalk (2011) directly address how the rapid normalization of worldwide networked communication has also aided those who prey upon minors:

The Internet allows...instant access to potential child victims worldwide, disguised identities for approaching children, even to the point of presenting as a member of teen groups. Furthermore, the Internet allows potential offenders ready access to chat areas and social networking sites reserved for teenagers and children, to discover how to approach and who to target as potential victims. The Internet provides a means to identify and track down home contact information, and the Internet enables adults to build long-term virtual relationships with potential victims, prior to attempting to engage the child in physical contact. ( $\mathrm{p}$. 25)

Gottschalk (2011) similarly outlined several characteristics of the Internet that make it an ideal site for grooming and soliciting minors. Most pertinent to the current study are those that are specifically communicative in nature. The first is disconnected personal 
communication - an effect of Internet communication that results in personal

discussions not being viewed as such— which results in lower inhibitions toward sexualized chat. This is also known as individuation (Berson, 2003), disentanglement from the body (Jewkes, 2010), or the online disinhibition effect (Whittle, HamiltonGiachritsis, Beech, \& Collings, 2013). Whittle et al. (2013) detail how certain features of the Web are key to this concept, such as perceived anonymity, asynchronous communication, and dissociative imagination, the cognitive separation of online fantasy from offline consequences. The online disinhibition effect can be understood as working in two opposing but sometimes simultaneous directions: benign disinhibition, or willingness to share personal emotions and details; and toxic disinhibition, the propensity to escalate conflict and angry/threatening sentiments. Both aspects of this bidirectionality focus on actions that people may perform online that they would not face-to-face. This idea ties in to Walther's (1996) hyperpersonal communication theory, which posits increased likelihood to self-disclose more and with greater detail online than in person. The aforementioned features of the Internet, as well as greater perceived message control, have been suggested as the roots of this phenomenon. According to Davis (2012), a majority of adolescents interviewed said they felt it was easier to be open about themselves and their emotions online than offline. This becomes salient when considering the trust-building and grooming strategies utilized by adults seeking youth in chat rooms, wherein the end goal is to be perceived as a trusted friend rather than a stranger ${ }^{4}$.

Gottschalk's (2011) list of relevant Internet features then moves on to discuss the mediation, universality, and time moderation of $\mathrm{CMC}$, or the ways in which online

\footnotetext{
${ }^{4}$ See 'Cybergrooming' subsection.
} 
communication connects potentially disparate parties anywhere at any time. The Internet can also serve as a distribution channel for products and information; this is important because groomers can send gifts and materials to a child that the child either desires (i.e. gifts) or does not desire (i.e. pornography). The Web allows for the formation of the electronic double and its subsequent manipulation, meaning that the persona inhabited by an individual online is fluid, often inaccurate or incomplete, and easy to alter. It is important to note that the electronic double effect is also bidirectional: "Also, the man may perceive the child and create an electronic double of the child in his head, which can be far away from reality" (p. 449). This is relevant to the present context because studies have shown that the Proteus effect, the idea that fluid online identitiesand the behavioral changes they theoretically cause - can measurably impact online communication. For example, young people who use sexier avatars or present their online identities as sexualized or provocative are more likely to experience sexual conversations online or be solicited by those they met in this space (Whittle et al., 2013).

The aforementioned features are useful as a backdrop for beginning to understand online predation, but they lack specificity in terms of the site at which this phenomenon actually takes place. As the present study is specifically concerned with analyzing chat transcripts between child groomers and individuals they believe to be underage, a brief outline of chat room features that make these spaces ideal for predation follows.

Chat room features. Berson (2003) called chat rooms "the new playgrounds for youth and the pedophiles stalking them" (p. 8). According to Malesky (2007), 81\% of interviewed online groomers admitted they visit chat rooms geared toward minors to identify and contact potential victims. This figure dwarfs the nearly half who admitted 
CYBERGROOMING

viewing children's online profiles to gather information and less than $10 \%$ who utilized public posts like those on blogs. Similarly, Briggs, Simon, and Simonsen (2010) found that all online groomers within their sample utilized chat rooms in this manner. Marcum (2007) noted how assumed anonymity might lead youth to seek chat rooms and participate in personal or explicit conversation: "Children engaging in sexual discussion feel more mature during these conversations, but also feel safe by believing their true identity is unknown. However, during conversation, children begin to trust their adult online companions and reveal an extensive amount of information" (p. 102). This perceived anonymity works both ways, because adults may also hide behind this guise (Briggs et al., 2010; Malesky, 2007; Olson, Daggs, Ellevold, \& Rogers, 2007; Whittle et al., 2013).

In general, chat rooms are common sites for sexual solicitation because they facilitate direct, instantaneous communication, and many chat rooms - particularly ones frequented by adolescents - are known to regularly house sexually explicit or otherwise obscene language and ideas (Subrahmanyam, Smahel, \& Greenfield, 2006; Wolak et al., 2008). Wolak et al. went on to note that youth who regularly chat are more likely to have many of the personality traits that make them ideal candidates for sexual exploitation (e.g. loneliness, poor relationships with parents, lacking in social skills, etc.).

Furthermore, a youth mentioning sex or appearing willing to discuss the topic is one of the top cues potential abusers utilize to identify willing victims (Malesky, 2007). However, this topical area does not have to be discussed directly with the adult; since chat conversations are public unless specifically set as private, sexualized conversations between teenage peers can also be cues as to which will most likely be open to sexual 
advances (Malesky, 2007; Webster et al., 2010). Chat room communication also lacks the presence of interpersonal aspects of conversation that are salient face-to-face and important measures of true intentions; this can lead to reduction of "stranger danger" fears as long online conversations rife with self-disclosure can lead young people to view their chat partner more as a peer than as a stranger with unknown objectives (Berson, 2003).

Chat rooms can also go beyond simple topical conversations and move toward acting out fantasies via cybersex or role-play that some perceive as a substitute for, or predicator to, offline acts against children (Quayle \& Taylor, 2003; Quinn \& Forsyth, 2004). The multidimensionality of the Internet is also an important factor. Briggs et al. (2010) discovered that over two-thirds of online sex offenders sent nude photos of themselves to the child during chat. Additionally, pornographic material may be transmitted in order to desensitize the victim, gauge their limits, or visually introduce sexual desires. For example, Webster et al. (2010) reported that the nature of the pornography sent by the perpetrator often coincides with subsequent sexual requests of a similar nature to that portrayed in the obscene photos or videos. The anonymity, ubiquity, and tendency for disinhibited communication that characterize chat rooms, as well as the fact that they are routinely frequented by youth, make them ideal locales for predatory online behavior - and also rich sources of naturally-occurring predation that can be analyzed. As such, the features and dynamics of the Internet, and chat rooms specifically, will underlie the present study.

Youth online. While usage and demographic numbers vary between studies, it is widely accepted that a large majority of people under the age of 18 regularly use the 
Internet, chat rooms, and social networking sites, all of which allow them to communicate with unlimited numbers of others as well as divulge personal information (Davidson \& Gottschalk, 2011; Davis, 2012; Gottschalk, 2011; Jewkes, 2010; Marcum, 2007; Whittle et al., 2013; Wolak et al., 2008). Adolescents take advantage of this wideopen communicative arena. In fact, Subrahmanyam and Greenfield (2008) found that $25 \%$ of teen Internet users have formed casual friendships with those they met online, and $14 \%$ reported forming close friendships and/or romantic relationships. Whittle et al. (2013) further stated that, for youth, Internet use rises with age, as does the variety of places from which it is accessed (away from home becoming more prominent as children age). As the present study focuses on chat room communication, it is significant that almost all youth who are online participate in chat (Davidson \& Gottschalk, 2011; Gottschalk, 2011). Furthermore, several studies (Marcum, 2007; Mitchell, Wolak, \& Finkelhor, 2005; Quinn \& Forsyth, 2005; Shannon, 2008) have found that $20-25 \%$ of youth reported receiving sexual advances or being exposed to unsolicited sexual material while online, and a majority of these victims were girls. Finally, according to Wolak et al. (2008), 99\% of victims of sex crimes initiated within cyberspace were between the ages of 13 and 17; thus since Perverted Justice decoys looking to expose online predators pose as teens in this age bracket, their conversational data serves as a unique window into naturally-occurring cybergrooming discourse.

Research has also shed light upon which youths are most likely to be victimized online. While girls are more likely than boys to experience online victimization, a perhaps more useful finding is that young people who engage in more risky behavior are more vulnerable, as well. Specific to risky online behavior, Wolak et al. (2008) found that 
older adolescents (15-17 years old) were more likely than their younger peers to disclose private information and converse with strangers online. In fact, these authors noted that as the number of risky behaviors (such as seeking pornography, talking to strangers, having sexually explicit chat, etc.) rises, so does the chance of interpersonal abuse online. Subrahmanyam \& Greenfield's (2008) survey revealed that $40 \%$ of young people on social networks have been contacted by a stranger online, and $41 \%$ of those who formed relationships went on to meet the stranger in an offline setting. Talking with unknown persons seems to be the agreed-upon "riskiest" action, and combining this with other forms of dangerous online behavior makes one much more likely to be victimized (Wolak et al., 2008). Young people, even pre-adolescents, have been shown to be very willing to have sexual conversations online. Over half (53\%) of 8-11 year olds admitted chatting about sex (Marcum, 2007). This is important because, as Malesky (2007) found, the most common factor in victim selection according to convicted online predators was the child mentioning sex. The aforementioned online disinhibition effect (Whittle et al., 2013) can make young people more likely to experiment by participating in sexually explicit chat and/or exposing themselves online. This, in turn, can cue potential abusers lurking for victims.

These Internet and chat-related features and phenomena are what make the process of grooming a child online similar in some ways, and yet different in others, from traditional notions of child predation. This relationship is only beginning to be explored. Below, a review of traditional (offline) grooming theory as compared to related practices observed in cyberspace reveals some uncertainty as to what extent the existing framework fits the new medium. 
Luring and Grooming Theory

According to Olson et al. (2007), child sexual abuse can be defined as an abuse of power by the adult with the intention of achieving sexual gratification with a child.

Finkelhor (1997) set three conditions under which the sexual abuse of a child takes place:

(1) "when a large age or maturational difference exists between the partners"; (2) "when

the partner is in a position of authority over or in a care-taking relationship with the child"; and (3) "when the acts are carried out against the child by using violence or trickery" (p. 101). Finkelhor delineated four preconditions necessary for child sexual abuse to occur. The first is motivation, which must include the ability to be sexually aroused by the child and may be amplified by unmet emotional and sexual needs. Second, the perpetrator must be able to disregard moral overtures against sexual relationships with children and overcome fears of potential legal consequences. Third, access to the child must be gained; and fourth, the offender ${ }^{5}$ must overcome the resistance of the child either by aggression or manipulation (or a combination thereof). While preconditions such as motivation cannot be established short of interviewing child sex abusers to elicit their internal thought processes in their own words, these other factors may be salient within my study's conversational data set. For example, these abusers' propensity to attempt a realignment of the victim's moral overtures against adult-child relationships (McGhee et al., 2011; Olson et al., 2007) could be seen as an attempt to overcome both his own and the victim's moral inhibitions. Before delving into luring theory and grooming literature

\footnotetext{
${ }^{5}$ Note on terminology: Henceforth 'sex offender' shall solely be used to denote individuals who have been convicted of sexual crimes of any nature, as opposed to 'child sex abuser' and the like, which will signify one who has sexually abused a child, specifically. Conversely, terms such as 'groomer' shall connote an individual who performs specific communicative/behavioral actions that conceptually mark them as such, sans the aforementioned legal nuances. Finally, 'perpetrator' will refer to one who performs actions conceptually salient to the 'wrongness' or deviance of sexually abusing a child (communicatively or physically).
} 
in detail, the following subsection will briefly present what is known about child sex abusers and their victims as individuals.

Perpetrator and victim characteristics. The majority of academic work concerning sex offenses against children has focused on characteristics of offenders and their victims. An overwhelming majority (90\%) of sex offenders who target children are men (Finkelhor, 1997). Though child sex abusers are a heterogeneous group, most studies find that the average male offender tends to be in his 30 's or 40 's. Otherwise, few patterns have been found in terms of offenders' education level, employment type, marital status, etc. (Deslauriers-Varin \& Beauregard, 2010; Elliott, Browne \& Kilcoyne, 1995; Lang \& Frenzel, 1988). As the data for the present study do not consistently provide such information about the adult involved in chat, it will be more useful to concentrate on what types of youth are most vulnerable, as Perverted Justice decoys are trained to take on this role (pervertedjustice.com).

Reported victim demographics vary between studies, but some personality characteristics seem to make certain youth more targetable. Elliott et al. (1995) found that $58 \%$ of convicted child sex abusers targeted exclusively girls, compared to just $14 \%$ for boys and $28 \%$ for both sexes. More predictive than victim demographics are their social and emotional characteristics. Researchers overwhelmingly agree that individuals who target children for sexual assault seek out victims who have low confidence, low selfesteem, passive disposition, emotional dependence, and/or are open to sexual experimentation (Elliott et al., 1995; Lang \& Frenzel, 1988; Robertiello \& Terry, 2007; Walsh \& Wolak, 2005). Children with single parents, low levels of parental 
involvement/supervision, or poor parent-child relationships are also more at-risk for victimization (Olson et al., 2007; Robertiello \& Terry, 2007).

Finally, according to Deslauriers-Varin and Beauregard (2010), likelihood of victimization (for any interpersonal crime) can be modified by victims' actions and lifestyles that may lead them toward or away from danger: "Lifestyle behaviors and characteristics, over and above proxies of lifestyle such as demographics and victim characteristics, are determinant in crime-commission and target selection" (p. 319). This lends credence to the idea that sexual predators choose specific locations such as parks (offline) and chat rooms (online) with a high number of potential victims and lower probability of close monitoring by guardians. Now that the context surrounding perpetrators and victims has been outlined, it will be useful to examine a few models that have been created in attempts to typify the process of sexually abusing children, as well as studies that have tested emergent theories and/or added to knowledge on this topic.

The luring process. The present study was informed by prior research concerning luring in its sexual context. However, similar processes can be used, for example, by con artists, gangs, and cults, to recruit victims/members (Olson et al., 2007). Luring, for the purposes of this paper, can be defined as the process by which an individual coerces, entraps, and/or manipulates another individual for the purpose of initiating/maintaining a sexual relationship (Campbell, 2009; Lang \& Frenzel, 1988; McGhee et al., 2011; Olson et al., 2007). This type of luring can involve any combination of aggressive force, threats, and subtle manipulation, and can often be achieved without any force at all. In fact, early studies investigating the sexual abuse of children found that aggressive force and/or threats were used by the perpetrator anywhere from $20 \%$ to more than half of the time 
(Lang \& Frenzel, 1988). This suggests the prevalence and effectiveness of more subtle, non-violent luring strategies.

Lang and Frenzel (1988) and Elliott et al. (1995) were among the first to utilize qualitative interviewing of sex offenders who targeted children. Although much of this literature makes it clear that the majority of this type of crime is perpetrated by someone the victim previously knew, the present study sought to highlight, where possible, the primary strategies used by strangers (because this study will focus on adults who look to lure children in chat rooms, where participants rarely know each other offline before conversing). Prior to the advent of the Internet, however, the locality of victim recruitment was a very salient aspect of luring. Deslauriers-Varin and Beauregard (2010) explain victim recruitment via routine activities theory; through this lens, a perpetrator selects a victim based upon three converging conditions: motivation, suitable target, and absence of a guardian. The latter two are relevant here to the overall process of initiating contact and subsequent luring. Elliott et al. (1995) found that 35\% of offenders previously unknown to the child searched locations which children frequent, such as schools, shopping centers, playgrounds, etc. It is notable that most offenses do not happen in these public locations, but rather in the home of the child or perpetrator. These interviews found that the most common strategies child sex abusers admitted to was attempting to gain access to the family home and/or have private time with the child (e.g. offering to babysit or teaching/coaching a skill or sport). Other common ploys included bribery, special trips, and the use of affectionate and empathic language (Campbell, 2009; Elliott et al., 1995; Lang \& Frenzel, 1988). These approaches all either lead up to an initial offense or are utilized to maintain the relationship for subsequent abuse, and most 
importantly, attempt to isolate the child from his/her guardians. In terms of privacy and isolation, the anonymous and unmonitored features of the Internet and chat rooms have removed, at least communicatively, the need for physical isolation in early stages of grooming ${ }^{6}$. This again highlights the need for research into how online communication has changed luring strategies and how they can be detected in their new form(s).

Once the child is isolated, strategies for initiating sexual contact can take many forms. However, the fact that a large majority (84\%) of child sex abusers said they subsequently re-used strategies that had previously been successful, suggesting that luring behavior becomes a discernible pattern (Elliott et al., 1995). Both Elliott et al. and Lang and Frenzel (1988) discovered that the most common tactic for initiating sexual contact was the adult "accidentally touching" the child, then gauging his/her reaction. According to Elliott et al. (1995), a majority (61\%) of offenders said that if the child reacted negatively they would stop the behavior and then try to subtly convince the victim that nothing was wrong before attempting physical contact again (as compared to $39 \%$ who said they were prepared to use force): "Therefore, the majority of offenders coerced children by carefully testing the child's reaction to sex, by bringing up sexual matters or having sexual materials around, and by subtly increasing sexual touching" (p. 585). Other common tactics included misrepresenting moral standards by suggesting, for example, that this type of play was normal or that it would make them more desirable partners in the future; framing the sex act(s) as education; and showing empathy, love, and affection (real or feigned) to an emotionally vulnerable child. A final tactic that must be noted here is how the adult convinces the child to keep the abuse secret, which can run

\footnotetext{
${ }^{6}$ See subsection on Internet features for more a more detailed evaluation of the Internet as a tool for luring.
} 
the gamut from passive manipulation to overt threats. Common examples include bribery and/or the communication of the act as a "special secret," non-physical threats to the victim's family dynamics, and threats of violence toward the victim or other family members (Elliott et al., 1995; Lang \& Frenzel, 1988). This facet of the pattern not only adds another layer of manipulation but also indicates that a high number of perpetrators knew what they were doing was wrong and thus were attempting to protect their own social and legal interests. These are all behaviors that rely heavily on successful communication strategies, which may or may not be evident in computer-mediated communication (CMC). In addition, before the advent of the Internet, these studies had to rely upon interviews rather than naturally-occurring data. My study sought to fill this gap by developing a revised framework based on what groomers are actually "doing" communicatively while chatting online with youth.

Luring communication theory. Olson et al. (2007) formulated luring communication theory (LCT), one of the most comprehensive explications of the behavioral and communicative processes behind the sexual abuse of minors. The authors state that the act of pursuing/soliciting a child for sexual purposes begins with the causal condition of gaining access to that child. Gaining access has three properties: the individual characteristics of the perpetrator and the victim, as well as strategic placement. Individual characteristics of both parties have been reviewed in detail above. The authors also point out that abusers are adept at identifying vulnerable youth. Finally, strategic placement refers to the perpetrator specifically targeting locations from which to access potential young partners (Olson et al., 2007). However, as mentioned above, the Internet has removed the initial access barrier by allowing instant communication to anyone, 
anywhere. In a sense, the strategic placement aspect of gaining access is nullified or has evolved to encapsulate virtual space. Therefore, it may be useful to think of strategic placement as it applies to the present study as chat room selection. Though this technology does provide a sort of buffer zone between perpetrator and victim in terms of physical contact, it potentially further highlights the communicative aspects of luring in the absence of physical coercive acts like the accidental touch and, in some cases, threats of physical harm. Thus, especially in CMC, luring leans heavily on communicative strategies and the dynamics of synchronous chat interaction.

Once access has been gained, the Olson et al. (2007) framework moves on to what the authors dubbed the cycle of entrapment: "We propose that the child sexual abuser has the uncanny ability to reconstruct the child's sense of self, modify the child's notion of right and wrong, and reduce feelings of agency" (p. 240). The core tenet of LCT is this deceptive trust development, defined as: "The perpetrator's ability to cultivate relationships with potential victims and possibly their families that are intended to benefit the perpetrator's own sexual interests" (p. 240). Through this process—which involves action strategies like paying extra attention to the child, bribes and gifts, and showing high levels of empathy — a sense of trust is falsely construed. In other words, the perpetrator's goal is to establish a bond such that the child sees him as a trusted and compassionate friend and/or authority figure. Three constructs: grooming, isolation, and approach, are the primary associated acts. As grooming itself is most relevant to the present study, the following subsection will define and explore this process. It should be noted again, however, that most of this theory was developed based upon self-report and interview data as there was no way to observe this behavior as it naturally occurs. As 
such, they acted as sensitizing concepts for my study's inductive approach, which was designed to counter this issue and revealed some (but not all) of these processes.

Grooming. Sexual grooming is the communicative aspect of luring theory, and thus a relevant framework with which to examine online sexual predation. While the Internet has certainly widened the theoretical scope of grooming, such emerging frameworks are grounded in classic, offline luring theory. According to Berson (2003), relationships between adults and children feature "an imbalance in power in which friendship and intimacy are leveraged for sexual interaction" (p. 11). In general, child grooming is understood to be a process of manipulation involving trust-building with, and desensitization of, the target child. The idea is that children are not adequately prepared to recognize such deceptive communication strategies; therefore the goal is to gain the victim's trust through the eliciting and sharing of personal - often sexualdetails (Berson, 2003; Craven, Brown, \& Gilchrist, 2006; Davidson \& Gottschalk, 2011;McGhee et al., 2011; Shannon, 2008; Whittle et al., 2013).

Exact definitions of sexual grooming vary within the literature. Within the LCT framework (Olson et al., 2007), grooming is defined as: "the subtle communication strategies that child sexual abusers use to prepare their potential victims to accept the sexual contact” (p. 241). These authors further break down grooming into two separate but related properties. The first, communicative desensitization, refers to the process of normalizing sexual subject matter and contact in the mind of the victim. This can be done physically or psychologically (i.e. 'sex education' or exposure to pornography). The second property is called reframing. This involves implicit sexual cues rather than direct verbal approach/request with the intent of preparing the victim for sexual contact. For 
example, a perpetrator might reframe the sex act as a game or suggest that it is a normal, healthy activity despite society's abhorrence of such behavior.

A thorough literature review by Craven et al. (2006) led to the following definition of sexual grooming: "A process by which a person prepares a child, significant adults and the environment for the abuse of this child" (p. 297). Three types of grooming have been outlined within this context: Self-grooming, through which adults attempt to justify or deny the immorality/illegality of their actions; grooming the environment and significant others, meaning gaining a physical presence and/or trusting relationship with nearby places and authority figures; and grooming the child, involving desensitization to sexual advances and more trust-building strategies (Berson, 2003; Craven et al., 2006; McGhee et al., 2011; Whittle et al., 2013). Grooming the child can be further broken down into physical and psychological grooming, where the latter is used to gradually and manipulatively sexualize the relationship. According to Craven et al. (2006), the perpetrator may utilize such strategies as: offering his/her revised sexual education (usually hinting that sex with an adult is acceptable behavior, despite societal norms), building trust through sharing and eliciting of personal details prior to pushing boundaries, desensitizing the child, offering gifts, and using threats. Such behaviors attempt not only to convince the child to accept sexual advances, but also to reduce the risk that the child will report inappropriate behavior.

According to the LCT framework, another important, co-occurring process is isolation of the child both physically and mentally (Olson et al., 2007). Physical isolation in the offline context is self-explanatory and is supported by the aforementioned finding that child molesters often do this by offering to teach, coach, babysit, etc. (Elliott et al., 
1995; Lang \& Frenzel, 1988). Mental isolation refers to separating the child

psychologically and emotionally from everyone but the perpetrator, whose aim here is to "create or enlarge the mental space between the targeted victim and the victim's support network so that the perpetrator can then step into that space" (Olson et al., 2007, p. 243). Grooming and isolation lead then to the final process in the LCT cycle, approach. The approach stage is the point at which the perpetrator verbally or physically advances sexually. Here, the verbal aspect is seen as a precursor to a physical approach, though again this dynamic is likely altered in CMC.

Olson et al. (2007) also noted that deceptive trust development is often used exclusively on the child (because the parent is not present/aware) in CMC scenarios, which are the focus of this study. According to Olson et al., the purposes (trust-building, desensitization, and power and control over the child) of this type of communication remain consistent whether used in an offline or online setting, even though specific grooming strategies may differ for the latter (McGhee et al., 2011; Whittle et al., 2013). Consistently missing from these studies is analysis of actual utterances made during the grooming process, impossible until the advent of the Internet. As such, the following section will review extant literature that specifically investigates the grooming of children for sex online_-otherwise known as cybergrooming. This provides more sensitizing concepts to compare with naturally-occurring conversational data and highlights the fact that much of this research still relies on looking for data that match claims made in the literature, rather than inductively pursuing new theory. 


\section{Cybergrooming}

Cybergrooming is a process at once similar to, and different from, offline luring and grooming. One significant difference is that cybergroomers often do not conform to typical understandings of pedophiles ${ }^{7}$ (Briggs et al., 2011; Walsh \& Wolak, 2005; Whittle et al., 2013; Wolak et al., 2008). According to Briggs et al. (2011), those who target adolescents online differ from pedophiles in that their victim selection is based on sexual maturity and teens' natural curiosity about, and inexperience with, sexual acts. Individuals with this attraction to adolescents have been called ephebophiles (teen boys) or hebephiles (both sexes) (Briggs et al., 2011; Wolak et al., 2008). Although media often promulgate the stereotypical "dirty old man" persona for online groomers, research has shown that this group of individuals is much more heterogeneous (Jewkes, 2010). Mitchell et al. (2005) found that adults arrested for cybergrooming adolescents - whether or not their attempts resulted in a physical sex act — tended to be older, better-off socioeconomically, and less deviant and violent overall than the traditional mould would indicate. While many of the typical strategies for sexual grooming still apply online, emergent research has attempted to elucidate an online-specific typology, with mixed results.

Intentions and goals. Research has identified two goals potential child sex abusers may hope to accomplish via cybergrooming. While many do indeed attempt to set up an offline meeting subsequent to online interaction, some are content to limit the fantasy to the cyber sphere (Briggs et al., 2011; Marcum, 2007; Shannon, 2008). This latter type of cybergrooming allows some offenders to justify their actions with the

\footnotetext{
${ }^{7}$ Pedophiles are adults who sexually target prepubescent children who have not reached sexual maturity.
} 
notion that no physical harm is done to the child: "Their sexual desires are satisfied from the knowledge that they are discussing sexual acts with a young child, but believe they do not require further pursuance of any physical act" (Marcum, 2007, p. 103). Similarly, Briggs et al. (2011) classified online groomers into two behavioral groups: contact-driven and fantasy-driven. Contact-driven individuals were found to be motivated by the prospect of meeting the child offline for a physical extenuation of their online sexual relationship, while fantasy-driven groomers relied on cybersex and exhibitionism for gratification, limiting the interaction to the Internet. Both are considered potentially harmful to victims (Longo \& Groth, 1983). The present study was not particularly concerned with cybergroomers' final intentions but rather how they negotiate CMC to systematically groom victims. To this end, the cybergrooming process is equally pertinent regardless of intentions.

Cybergrooming practices. O'Connell's (2004) outline of chat room grooming practices named cybergrooming as a subset of cyber-sexploitation, the practice of adults chatting with children in sexually-charged language and/or participating in cybersex ${ }^{8}$. The key difference here is that conceptually, cyber-sexploitation is the involvement of a minor in an online fantasy act (i.e., cybersex), whereas the act of cybergrooming involves the subtle manipulation/trust-building outlined previously in the sexual grooming literature and is often associated with intent to physically abuse the victim. The subsequent typology outlines several progressive stages often (but not always) followed by cybergroomers. Beginning with the friendship-forming stage (O'Connell, 2004), the

\footnotetext{
${ }^{8}$ Cybersex can be defined as "a form of fantasy sex, which involves interactive chat room sessions where the participants describe sexual acts and sometimes disrobe and masturbate" (Briggs et al., 2011, p. 80).
} 
adult attempts to form a bond with the child in the interest of gaining his/her trust; it is in this stage that the groomer will often move the conversation from a public chat room to a private, one-on-one chat. The relationship-forming stage is an extension of the friendship-forming stage, during which the adult typically engages in the grooming strategy of trust-building, including gathering personal details and creating "an illusion of being the child's best friend" (p. 6) by continuing to ask (not necessarily sexual) questions and appearing to genuinely care. O'Connell was careful to note that not all online groomers follow this typology, and its stages often repeat or overlap. Following relationship-forming is the risk-assessment stage, marked by a shift toward questioning the child's trust and ability to keep a secret.

O'Connell (2004) noted that this stage very often acts as a bridge to more sexually explicit conversation; thus the sexual stage often begins with questions about what the child has/has not experienced sexually. The sexual stage is a crucial step in the process of cybergrooming as it is here where prior trust-building attempts are especially important: "The 'you can talk to me about anything' is a relatively [staple] part of the conversations of those adults who intend to maintain a longer term relationship and for whom the child's apparent trust and love is a vital part of their fantasy life" (p. 7). Here, the power aspect of child grooming (Berson, 2003; Finkelhor, 1997; Olson et al., 2007) becomes especially salient as the adult is much more likely to be able to effectively navigate this type of subject matter than is the child. O'Connell (2004) goes on to explain the typical progression of cybergrooming via three types of fantasy enactment, which lie on a spectrum that increases in intensity. The first, fantasy enactment based on perception of mutuality, is a common tactic in which the groomer will utilize the trust and perceived 
mutual understanding to directly invite or indirectly coerce the child into participating in cybersex. Alternatively, some cybergroomers are much more overt about their desires and more direct in requests for cybersex acts, but still offset this by maintaining the sense of friendship that was already established. This is known as fantasy enactment, or overt coercion counterbalanced by intimacy. Finally, some cybergroomers get right to the point, participating in cyber-rape fantasy, which is marked by "overt coercion, control, and aggression" (O’Connell, 2004, p. 9). Again it must be noted that groomers can utilize these stages and types of approaches intermittently, simultaneously, and with varying speed in progression. The present study sought to mitigate some of the uncertainty surrounding this novel phenomenon; some of these stages and behaviors were be apparent in the data, some were not, and new ones emerged.

McGhee et al. (2011) also extended the grooming model to fit online predation. Although their study's intent was to develop a computer algorithm to detect grooming (resulting in generally low accuracy), they modified Olson's (2007) model, dividing cybergrooming into four useful subcategories: (1) personal information; (2) relationship details; (3) activities; and (4) compliments. Personal information can include details about physical locations, contact information, birthdays, photographs, and any other pertinent personal info (such as the location of the victim's computer within the house). Relationship details and activities can range from everyday practices to sexualized questions of varying detail. Finally, compliments generally involve rapport-building by the groomer uttering positive/flattering appraisals of the child's responses to detail and activity questions, and are "intended to make the victim view the predator in a positive 
light" (p. 105). Aside from compliments, these all involve asking the child

progressively more personal questions, generally leading up to those of a sexual nature.

Whittle et al. (2013) refer to this as rapport building, during which the adult generates commonality with the minor by appearing to talk like them and/or questioning them on their interests, beliefs, personal details, etc. Also notable is that whether or not this stage of grooming leads to physical sexual contact, it does function as a desensitizer for the child ('communicative desensitization'), gradually making him/her more receptive to sexualized language: "Successful grooming leaves the victim unaware that any process is under way" (McGhee et al, 2011, p. 105). Communicative desensitization online can involve repeated use of vulgar or sexualized language/images (including transmitting pornography to the child) with the intent of getting them accustomed to sexual topics (McGhee et al., 2011; Olson et al., 2007). Another important aspect of grooming communication includes the reframing of sex acts and the adult's interest in the child as a positive so as to force the child to question society's norms (and view the perpetrator as a moral guide). Also, isolation - which on the Internet involves making sure the victim is chatting alone and emotionally isolating him/her to the point that a deep deceptive trust bond is formed — is a key facet of this process (McGhee et al., 2011; Olson et al., 2007). One commonality here that is worthy of investigation is the role of questions, which seem to be important tools that the predator can use in a wide variety of ways, such as establishing trust/rapport, gaining personal information, and advancing the conversation to a sexual level while the victim remains unaware of the grooming. However, no such utterance-level analysis has been performed on this type of data; as such my study works 
toward filling this gap by looking at groomers' utterances to determine what they accomplish and how their actions discursively manifest as cybergrooming behavior.

Buchanan (2012) specifically examined Perverted Justice transcripts between adults and children (the same data set from which my sample will be drawn). She utilized relational dialectics theory to identify three practices that online solicitors of minors use in discourse. One practice was called discrediting of the distal, in short referring to the process of calling society's mores_-specifically those marking adult-child relationships as immoral — into question. This study also identified ambiguous talk as a common pattern, through which solicitors avoided the appearance of inappropriate behavior by indirectly introducing sexualized talk. Buchanan then discussed the strategy of discursively making the child feel like an adult; in this way the sexualization of that individual (child) is seemingly more like that of an adult. Finally, this author explored how the groomers co-opted the discourse in an attempt to reframe themselves as the teacher and the child as the student. An example would be exposing the child to pornography but framing it as an educational experience during which he teaches in a sexualized manner and questions the child on sexual details (either previous experience or "how would you..." type questions). Again, such behaviors as asking questions of the child seem to be a significant feature of the phenomenon, one that goes beyond desiring the actual answer and serves deeper communicative purposes.

Indeed, questioning the child emerged throughout much of the literature as a frequent and salient strategy employed by cybergroomers, particularly in the early stages of chat. However, this phenomenon has yet to be isolated for research. For example, Marcum's (2007) examination of three case studies utilizing Perverted Justice transcripts 
revealed that all three involved a repeated questioning of the 'child' as to their sexual experience and interests. Using one case as an example, she concludes: "He seemed to derive pleasure from dominating the conversation with his victim by questioning her about her sexual past and insisting he was aware of sexual misconduct from her past" (p. 111). Additionally, in a review of grooming-related crimes in Sweden, sexual questions were the most common type of communication detected (Shannon, 2008). Studies such as these are useful as sensitizers to some behaviors (i.e. questioning, complementing, etc.) that may emerge from the data. However, most take a more broad approach that speaks to overall intentions and end-goals of groomers, rather than inductively focusing on specific utterances and their roles in cybergrooming discourse. As such, the present study attempts to fill this gap in the literature by investigating groomers' utterances and their function(s) as social actions within chat conversation. In order to work toward an understanding of the nature of cybergrooming discourse, I utilized some conceptual tenets of the Conversation Analysis (CA) framework. Therefore, the following section outlines salient concepts within this approach and includes a focus on how specific utterances may achieve social actions.

\section{A Conversation Analytic Framework}

Understanding the nature of communication as a social behavior that is intentionally designed and implemented to accomplish some form of social action is a necessary first step to investigating online discourse. Specifically, it is important to examine how people communicate their desires and intentions via $\mathrm{CMC}$, as well as how they attempt to achieve certain social actions through text alone. This is especially true in the context of the present study, as many cybergroomers are conceivably attempting to 
achieve something more than a simple transfer of information. The question then becomes: How can we tell what these individuals may be trying to communicate by examining their naturally-occurring $\mathrm{CMC}$ discourse? How cybergroomers construct their discourse to achieve specific social actions, such as grooming or luring their victims to engage in sexual behavior with them offline, is crucial to understanding how to protect young people who communicate with others in online environments. As such, CA offers a conceptual framework relevant to understanding and investigating the social actions achieved via communication in a variety of contexts. Indeed, Segerdahl (1998) acknowledged the potential for CA to be a technique for mapping the characteristic features of conversation in a particular context for the purpose of learning about that context, as well as using the knowledge gained from such endeavors for tackling a variety of practical problems.

A CA approach is appropriate to the present study because I analyzed talk that, in most ways, conforms to the stipulations of the science. Namely, the chat transcripts are naturally-occurring samples of talk-in-interaction (though traditional CA mandates audio/visual analysis, the synchronous nature of chat room discourse is an everincreasing medium for conversation). CA also mandates that talk be naturally-occurring (as opposed to institutional), and also that talk is transcribed in detail (Robinson, 2013; Sacks, Schegloff, \& Jefferson, 1974; Sidnell, 2010). Chat dialogue conforms to both of these rules, as well. Thus, I utilized some of the principles of CA, outlined below, to discover what adults who have sexually-charged conversations with young people online are trying to achieve, or "do," via their communicative actions. 
The following subsection provides a brief overview of CA. It begins with a discussion of its foundational assumptions as well as the communicative functions and social actions achieved by everyday discourse. Next, because the literature suggested that questions are particularly salient in this context, I look at the role(s) and function(s) of interrogatives, what they accomplish in talk, and what types of answers they prefer. Additionally, a consideration how people accomplish social action through online communication, noting important similarities and differences between computermediated contexts versus face-to-face, will also be salient. This includes explication of how the stipulations of the turn-taking model and preference organization may differ in online versus face-to-face contexts. Finally, I apply these elements of CA to sexual predator-prey interactions online to examine how sexual predators may achieve specific social actions through conversation.

Conversation analysis. Sidnell (2010) defined CA as "an approach within the social sciences that aims to describe, analyze and understand talk as a basic and constitutive feature of human social life" (p. 1). Recipient design, the primary underlying foundation of $\mathrm{CA}$, refers to the tendency to construct talk "in ways which display an orientation and sensitivity to the particular other(s) who are the co-participants" (Sacks et al., 1974, p. 727). In other words, sentences and utterances are constructed, disseminated, and understood as forms of social action in particular contexts that are sensitive to those contexts (Goodwin \& Heritage, 1990). This principle speaks to the importance of contextualizing specific bits of talk because variations in setting, the nature of coparticipants, etc. may contribute to noticeable and measurable variations in subsequent discourse. Robinson (2013) sums up the goals of CA research with three basic questions 
this approach attempts to address: “(1) How do speakers make sense or make meaning when they talk and, similarly, how do listeners know what speakers mean when they talk?; (2) How does an utterance's meaning affect subsequent talk?; and (3) How does an utterance's meaning affect speakers' relationships with each other?” (p. 96).

Furthermore, based on this foundation, CA rests on several assumptions about talk that subsequently dictate how talk is structured: First, talk emerges from goals; second, talk is regularly used in everyday life; and, thirdly, talk reflects individuals' unconscious adoption of social rules (Holt, 2003). In order to achieve said goals, and negotiate them within naturally-occurring conversation, speakers must adhere to, and continually formulate, norms that are sensitive to the context of the speakers' relationship and the conversation in which they are engaged. This negotiation of norms, and consequently any analysis seeking to explicate them, must consider members' meanings - what words, utterances, and conversational cues signify contextually to the speakers themselves - during every step of research (Holt, 2003; Robinson, 2013). Successful conversation, then, features mutual orientation (Goodwin \& Heritage, 1990), or intersubjectivity, defined by Sidnell (2010) as, "joint or shared understanding between persons" (p. 12). These assumptions preclude various organizations, or sets of practices, for constructing turns of talk, producing sequences of actions, initiating particular actions like repair, etc. ${ }^{9}$ Most salient to the current study are inherent preferences present within talk and the normative structure of preferred and dispreferred responses to particular types of talk. Also relevant is the underlying idea that talk is both designed to achieve an

\footnotetext{
${ }^{9}$ For a full overview of CA see Sidnell (2010).
} 
action and can offer a clue as to the contextual relationship between speakers and how they make meaning from discourse.

Communication as social action. As stated above, the primary reasoning behind my utilization of the CA framework is that communication is overwhelmingly designed not only to transfer information between parties, but to achieve some sort of social action. The emphasis here is on how people use communication to "do" something, or to communicatively accomplish a goal, and how this process is a function of the context in which the conversation is taking place (Goodwin \& Heritage, 1990; Goody, 1978a; Goody, 1978b; Robinson, 2013; Sacks et al., 1974; Sidnell, 2010). Or, as Goody (1978b) posits: "In performing a given speech act a person intends not only to communicate a referential meaning but also to actively influence his hearer in some way" (p. 18). Goodwin and Heritage (1990) further noted that said forms of action are concurrently context-shaped, or informed by prior conversational actions, and context renewing, meaning that each action, in turn, shapes the subsequent context of the dialogue.

This brings members' meanings into the forefront of a CA approach, as communication can only achieve action if both members take what is said to mean the same thing. This also means that communication can imply a certain level of relationship between the two parties. For example, according to Robinson (2013), a question such as 'How are you feeling?' can carry with it an undertone of assumed relationship as the questioning party knows enough about the other's health to inquire in a very specific way. In turn, the recipient of the question replies based upon a contextual evaluation of the questioner's prior knowledge. As such, "Analysis must move beyond the isolated sentence to encompass the sequences within which the individual actions occur and 
where they are linked to each other" (Goodwin \& Heritage, 1990, p. 289). This is especially true for the present study, as the participants presumably have just met and have little to no contextual knowledge before their dialogue begins. According to Robinson (2013), conversationalists naturally lean toward agreement and cohesion with the other party: "Action tends to be designed so as to reduce relational damage and promote relational bonding" (p. 105). In other words, speakers and hearers prefer certain types of responses, specifically ones which facilitate cohesion and a closer relationship with each other (Curl \& Drew, 2008; Goodwin \& Heritage, 1990; Pomerantz, 1984). Thus, a CA framework must consider how parties in discourse are normatively driven to respond in a preferred manner or account for not doing so.

Preference. According to Sidnell (2010), the idea of preference within conversation involves the concept of recipient design, or how a speaker's talk is constructed so as to make it relevant to the intended recipient. This, in turn, not only normatively forces the listener to respond, but also governs what types of responses are expected in order to promote social solidarity. The appropriate response is referred to as conditionally relevant. The adjacency-pair concept is particularly important to this discussion as it implies that any action introduced by a speaker, or first pair part, requires the next speaker to produce a reciprocal action, or second pair part. Therefore, the presence or absence of a conditionally-relevant second pair part can consequently alter the speaker's future utterances. Simply put, people construct and initiate actions with their words, which subsequently influence the communicative actions of others with whom they are conversing (Goodwin \& Heritage, 1990; Schegloff, 1968; Sidnell, 2010). 
The driving force of this process is preference organization, a component of

CA that is particularly salient to my investigation of how sexual predators may construct their turns to achieve specific goals. Because the basic premise of CA is that social interaction is built with a bias toward social solidarity and against social conflict, the organization of communication must be built to maximize politeness, friendliness, and affiliation (Curl \& Drew, 2008; Goodwin \& Heritage, 1990; Pomerantz, 1984; Sidnell, 2010; Steensig \& Drew, 2008). Thus, preference organization inherently dictates a set of normative responses to communicative actions that engender more affirmative relations. Various examples of pair types have been distinguished by CA in terms of the interactional sequences they initiate as well as the social actions they accomplish. Some of these sequences include: question-answer, greeting, request/grant, pursuit of response and invitation/acceptance/rejection (Curl \& Drew, 2008; Sacks et al., 1974; Schegloff, 1984; Sidnell, 2010; Tracy \& Robles, 2009). In terms of action and understanding, the adjacency-pair organization serves as a norm of conversation that guides interactional sequences and is used by speakers to hold their co-participants accountable during these sequences. Preference organization — specifically in the context of action-based preferences (e.g. asking questions, invitations, etc.) — mandates that first pair parts prefer specific and appropriate second pair parts. This phenomenon becomes extremely salient when considering question-answer sequences, which the literature suggests may be prevalent in the data. Thus, the following subsection briefly reviews literature on questions from a conversation analytic point of view.

Questioning as social action. Questions, as commonly understood grammatically, may not wholly encompass the broad range of utterances that can 
normatively demand a preferred, conditionally-relevant response. Therefore, the term 'interrogatives' better encompasses the action these types of utterances perform, whether or not they are grammatically or intonationally formulated as a question (Schegloff, 1984). According to Steensig and Drew (2008), grammatically, questions in the English language tend to take one of a few forms, such as a reversal of the subject-verb declarative format, often with the addition of an interrogative ('You are here.' vs. 'Are you here?'). They can also take the form of a declarative statement ending in a yes/no inquiry (i.e. 'You are here, aren't you?'). Conversely, open-ended questions are built as incomplete ideas, where the expected answer completes said idea and the clause/sentence holding it (i.e. 'Where are you?') (Goody, 1978b). As stated above, interrogatives do not have to take such obvious syntactic forms because phonetics (i.e. rising intonation) and pragmatics (having to do with the context and shared affiliation of the current conversation) can signify interrogative intention.

Apart from requesting information or the confirmation of such, questions can also serve diverse purposes such as inviting, making a request, heralding a new direction for conversation, indirectly making a complaint, or communicating a sense of power (Steensig \& Drew, 2008; Tracy \& Robles, 2009). Finally, the literature points to the constraining function of questions: "It is widely acknowledged that there is something compelling about questions_questions require answers" (Steensig \& Drew, 2008, p. 7). Asking a conversational co-participant a question dictates the immediate production of some form of answer, even if that answer is an explanation for why the next speaker cannot answer. Thus, interrogatives make relevant and expected an immediate and preferred reciprocation: Greetings dictate greetings (ex. 'Hello' / 'Hi') and requests or 
invitations dictate follow-up answers of either grants, acceptances, or rejections, respectively (Goody, 1978b; Schegloff, 1968; Sidnell, 2010; Steensig \& Drew, 2008; Tracy \& Robles, 2009). In this context, preferred responses are any answers that help speakers achieve their social actions, such as accepting invitations, granting requests, etc. Therefore, questions can exert significant discursive force, and the subsequent response (or lack thereof) can affect the rest of the conversation: "The conditional relevance that a question establishes ensures that participants will inspect any talk that follows a question to see if and how it answers the questions" (Sidnell, 2010, p. 63). Furthermore, Goody (1978b) points out that this system is governed by the contextual relationship between the speaker and listener: "Whether the reciprocity is equal or unequal is marked by the mode of questioning used, and is also a function of the relative statuses of questioner and respondent" (p. 23). This could become salient for the present study as the way adults structure interrogatives to individuals they perceive as children may provide a window into what type of relationship they are trying to build and how they go about communicatively achieving their goals in chat. The broad potential functionality of interrogatives in conversation necessitates looking past the obvious information-gleaning purpose of this type of utterance toward an explication of what social actions conversationalists are achieving when questioning.

Goody (1978b) begins with the view that questions - and indeed most types of utterances and linguistic tools - have both locutionary and performative functions. While locutionary function encompasses the more straight-forward, referential, informationseeking role of questions, the latter (performative) function refers to the social action a particular piece of conversation conveys or attempts to achieve. In other words, 
locutionary statements and questions can be shown as true or false, but this does not necessarily tap in to their function as social actions: "Performative aspects of speech acts are produced with certain kinds of intentions. Specifically, in performing a given speech act a person intends not only to communicate a referential meaning but also to actively influence his hearer in some way" (Goody, 1978b, p. 18). Furthermore, questions can carry report and command functions, where the former conveys referential information while the latter attempts to influence the future path of conversation. Goody (1978b) provides the useful example of a wife asking a husband what time it is at a party; this question can be seen as both a request for information and a hint that it is time to leave. In short, questions demand some sort of reciprocal action, whether it be a verbal response or an action of some sort: "It is this fact which leads to questions often carrying a strong command message" (p. 23). Finally, questions are temporally significant as they not only demand action, but immediate action (though synchronous $\mathrm{CMC}$ calls the immediacy of such temporal demands into question). Thus, immediate, preferred responses engender affiliation within relationships, while delayed, absent, or dispreferred responses can cause disaffiliation and usually require a further account in order to maintain face (Pomerantz, 1984; Steensig \& Drew, 2008).

Question-asking can also be a powerful tool for initiating and/or maintaining a particular topic of conversation. This is especially the case when a power differential is present, such as in a teacher-student or adult-child interaction, where the party who poses the question can hold their conversational partner accountable for answering (and doing so in the preferred manner) (Goodwin \& Heritage, 1990; Goody, 1978b; Tracy \& Robles, 2009). Goodwin and Heritage (1990) refer to discourse identities, or the assumed 
knowledge and expected normative behaviors that co-participants in a conversation expect each other to conform to. Under this view, for example, posing a question assumes that the recipient has the relevant information to answer the question and thus categorizes said recipient. This idea can be further extended to encompass the current and future topic(s) of conversation: "An inquiry about household matters may exhibit and make relevant to the action of the moment a domestic relationship between speaker and addressee" (p. 293). As such, the use of specific terms within a question predicate a certain level of knowledge on the part of the recipient, and thus makes relevant (and expected) a response that furthers this specific context. In terms of the present study, for example, the use of sexual language and questions regarding relationship details or sexual experience make relevant the same in return, also potentially establishing the relationship between the two speakers as a sexual one, or at least one in which the discussion of sex is normalized. In other words, once question-answer sequences of a sexual nature are initiated and preferred responses further said sequences, the discourse identities of the participants have been established and such personal questions may be seen as appropriate throughout the rest of the interaction. This is where introducing sexual topics into a conversation becomes a powerful tool for displaying intentions as well as establishing where the immediate future of discourse lies.

This brief overview of CA has revealed how conversation is simultaneously driven by context and shapes future conversation. Additionally, the focus on interrogatives as much more than simply statements that seek information, but as a discursive way to perform social actions, hold the other party accountable for response to specific inquiries, and imply some sort of relationship between parties, suggests that they 
can be a powerful tool in navigating discourse between individuals with an imbalance of power (such as an adult-child sexually-laden conversation). As such, the knowledge gap I address is how discourse facets such as preference and sequencing during naturallyoccurring cybergrooming affect the overall discourse and what they achieve contextually. However, as this study is concerned with how people achieve social action in synchronous online communication, the following subsection addresses extant literature on how the rules of conversation can differ when considering $\mathrm{CMC}$ as opposed to traditional, face-to-face, conversation.

Analyzing cybergrooming. Online communication differs from face-to-face communication in a myriad of ways. CMC has the dual capacity to be either asynchronous (e.g. broken up over time like in e-mails, Facebook messaging) or synchronous (e.g. continuous in time like in chat rooms). Synchronous online communication provides a text-based communication context that both adheres to and slightly modifies the theoretical bases and structures of CA. Simpson's (2005) application of CA to discourse in an online learning community revealed similar workings of the adjacency-pair sequence and preference organization in online chat. Synchronous online discourse is often plagued by what Herring (1999) calls disrupted turn adjacency, which refers to a loss of sequential coherence and manifests itself in written text in the form of improperly sequenced talk. Simpson (2005) suggests that this occurs because visual and auditory cues characteristic of spoken discourse are absent, which means speakers' turns cannot be read and oriented to until they are sent.

Instead, Simpson's (2005) results confirm previous research on the existence of the conversational floor as the primary source of coherence in synchronous online 
communication. In other words, this serves as the "glue" of social cohesion, which is a goal of naturally-occurring human communication. Edelsky (1981) defines the conversational floor as the "acknowledged what's-going-on within a psychological time/space" (p. 405). This floor has three primary elements, all of which must be inferred from the written text of an online conversation: the topic of discourse, or "aboutness," the communicative action of discourse, and the participants" "sense of what is happening" as identifiable from their written cues in the context of a particular bit of written talk (Simpson, 2005, p. 345). For the purposes of this study, the conversational floors created and navigated by sexual predators and their potential victims and how these are used to achieve some form of social action become salient. While the turntaking structure may be less impactful in structuring turns in online discourse, actionbased preferences are still evident, which suggests that preferred and dispreferred responses to actions undergird CMC. Furthermore, research has suggested that cybergroomers spend substantial amounts of time reading children's online profiles in order to find and study their potential victims (Deslauriers-Varin \& Beauregard, 2010; Malesky, 2007). This suggests cybergroomers understand the importance of knowing and orienting to contextual factors, specifically co-participants, when striving to achieve some form of social action through conversation.

Action-based preferences are pertinent to the study at hand as these are major tools that people use to achieve social action through conversation. As demonstrated above, CA suggests that social actions are embedded in the structure of conversation, meaning the way cybergroomers utilize such restraining utterances can drive the chat in a particular direction and potentially facilitate deceptive trust development and other 
aforementioned cybergrooming behaviors. As themes emerge from these

communicative actions, it becomes be possible to glean some understanding of what cybergrooming looks like for those who perpetrate it.

\section{Conclusion and Research Questions}

This review of literature has outlined Internet and chat room features salient to cybergrooming, sexual luring theory as applied traditionally and online, and the basic tenets of CA that guided my analysis. My exploration of these topical areas and concepts has revealed that more research must investigate how synchronous online communication has altered the dynamics of sexual luring; the studies that have emerged tend to rely too heavily upon seeking patterns associated with offline luring within online behavior. Interviews and self-reports (the basis for most cybergrooming studies) may be able to elucidate intentions and conscious strategies, but they miss the truly primal communication behavior that analysis of naturally-occurring conversation can highlight. Thus, the power of single utterances within discourse is a major theme of the present study. This is the view I took while exploring what cybergrooming means for the individuals who perpetrate it. Although the grooming literature offers some varying operationalizations of the phenomenon, as well as some conceptual deductions of how it manifests, no study to date has inductively examined what cybergrooming means to those who perform it, what this type of communication is 'doing' for the groomer, and how its implementation impacts the navigation of conversation during the act.

Therefore, the following research questions guided the present study:

\section{RQ1: In the context of online chat rooms, how do adults discursively orient} to and manage the process of cybergrooming minors? 
RQ1a: Are there systematic patterns in terms of the social actions that constitute cybergrooming?

RQ1b: Are there systematic patterns in terms of how cybergrooming is sequentially organized? 


\section{Chapter 3: Method}

\section{Identifying a Need for Qualitative Research}

As discussed below, the present study was guided by an inductive paradigm, including a qualitative epistemology and ontology (Becker, 2001; Morgan \& Smircich, 1980; Silverman, 2006). This section is devoted to identifying the need for qualitative research on cybergrooming, as the ultimate goal of this project was to conceptually describe this phenomenon based on naturally-occurring observations of behavior. A review of extant literature (see Chapter II) identified several theories in which the concept of cybergrooming figured centrally. For example, cybergroomers have been divided into fantasy-driven and contact-driven categories in terms of their intentions toward victims (Briggs et al., 2011; Marcum, 2007), though both have been found to be harmful psychologically to youth and potentially make it more likely that the victim will commit sex crimes in the future (Longo \& Groth, 1983). Prior research has also indicated that teens are relatively likely to be exposed to online sexual solicitation (Marcum, 2007; Mitchell et al., 2005; Quinn \& Forsyth, 2005; Shannon, 2008), and furthermore that certain types of risky online behaviors - such as talking about sexual topics during chat (Malesky, 2007) and disclosing personal details to strangers (Wolak et al., 2008)—can positively affect teens' likelihood of victimization. Therefore, the present study adopts cybergrooming as a sensitizing concept, or one that "lays the foundation for the analysis of research data" (Bowen, 2006, p. 3) by highlighting the presence of an important social/interactional construct. Theoretically, cybergrooming's explication is in its infancy, and more research is needed to add to and/or modify the corpus of behaviors associated with it. 
The preceding review of literature also alluded to myriad operationalizations of the concept of cybergrooming as a communicative process. For several examples, O'Connell (2004) suggested that cybergrooming discursively progresses from a friendship level to a sexual level, and Olson et al. (2007) put forth that cybergrooming is largely centered around adults deceptively forming trusting bonds with potential victims via such acts as showing heightened empathy, communicatively desensitizing the youth, and psychologically isolating him/her. Whittle et al. (2013) stated that cybergrooming involves building rapport through the enactment of questions and compliments that generate commonality. McGhee et al. (2011) suggested that cybergrooming involves the elicitation and disclosure of personal information, relationship details, and activities (often progressing from innocuous to sexual).

Although cybergrooming appears to be a theoretically important concept, one major critique of its prior operationalizations is that they have been primarily generated deductively, that is, without ample and systematic consideration of its actual features and processes as they occur naturally. Most such studies were not grounded in naturallyoccurring data (e.g. Olson et al. (2007), who performed a grounded theory meta-analysis of extant literature on the subject), and the few that did examine natural data (e.g. Buchanan, 2012; McGhee et al., 2011) still applied existing theoretical frameworks to their analyses.

\section{Qualitative Epistemology and Ontology}

This study was guided by a qualitative epistemology and ontology (Becker, 2001; Morgan \& Smircich, 1980; Silverman, 2006), and thus an inductive methodology. Specifically, this study was conducted with a symbolic-interactionist approach (Blumer, 
1969). Blumer outlined three principles of this qualitative epistemology. The first is that people behave toward things based on the meanings they have for those things. These "things" range from everyday objects to interactions with other people. Second, said meanings are not intrinsic; instead they are built through social interaction. Third, these meanings are not static, but constantly determine how individuals respond to situations and, in turn, modify meanings that people have for experiences. In short, through a process of constant interpretation of one's own and others' actions, meaning is created and negotiated through a process of social interaction. Following Blumer, this study focused on describing what cybergrooming means to individuals who perform it by analyzing the social actions discursively performed by cybergroomers and detecting patterns in how those actions are organized and sequenced. As Becker (2001) indicated: "The point is not to prove, beyond doubt, the existence of particular relationships so much as to describe a system of relationships, to show how things hang together in a web of mutual influence" (p. 319).

According to Silverman (2006), "Conversation is the primary medium through which social interaction takes place" (p. 203). The present study was guided by socialconstructionist ontology, or one which focuses on this process of meaning negotiation through the social action of conversation. In a microcosmic sense, reality is being perpetually constructed and modified by both parties in a conversation (Annells, 1996; Gergen, 1985). Morgan and Smircich (1980) look at discourse as the symbolic negotiation of reality: "The fundamental character of the social world is embedded in the network of subjective meanings that sustain the rule-like actions that lend it enduring form" (p. 494). Under this ontology, each utterance is significant in that it both draws 
CYBERGROOMING

from prior experience and has the power to influence future action. As Gergen (1985)

states: "The question 'why' is answered not with a psychological state or process but with consideration of persons in relationship" (p. 271). It is not a far reach to imply the inverse: The question "why" is also asked with consideration of the other party. Since social action is driven by perpetually-formulated knowledge, it follows that this study's analysis of said social action should be based on the discernible experiences and points of view of the actors within the data.

According to Becker (2001), qualitative researchers either observe behavior in situ or by means of self-report via interviews and the like, both of which have their disadvantages. In the former case, the researcher's presence naturally affects actors' behavior, and in the latter, self-reports can be notoriously unreliable/inaccurate. However, I sought to circumvent both of these issues, as this study's data was naturally-occurring chat during which no researcher was present. This represents a unique opportunity to glean novel emic descriptions - ones that describe a phenomenon in terms of members' meanings (Emerson, 2001) — of the communicative behavior taking place during these chats. As Emerson noted: 'Emic accounts are not literally members' constructs, but rather second-order renderings of those constructs" (p. 35). The present project tapped into what Geertz (in Emerson, 2001, p. 35) referred to as, experience-near concepts. Emerson (2001) described 'experience-near' as the other half of the continuum leading toward experience-distant description, the latter of which more closely mirrors etic concepts, or ones proscribed to a phenomenon based on preconceived notions and categories. 


\section{Grounded Theory}

Based on the present study's qualitative epistemology and ontology, it used the method of grounded theory. According to Glaser and Strauss (1967), grounded theory refers to the process of building theory from social research data. More specifically, this approach attempts to build guiding theory that is appropriate for examining a particular phenomenon without relying on a priori assumptions. One of the main strengths of this type of inductive method, as Strauss and Corbin (1998) pointed out, is that the emergent theory derives from observable data. Such theory should "enable prediction and explanation of behavior...be usable in practical applications...provide a perspective on behavior...and provide a style for research on particular areas of behavior" (Glaser \& Strauss, 1967, p. 3). The stance grounded theory takes, and the one to which I adhered for this project, is that theoretical concepts should be built systematically in relation to data. This places the actual process of research at the forefront of theory generation, and this process relies on the researcher's constant development of categories based on their observed properties, conditions and consequences. As Glaser and Strauss (1967) noted, categories and their properties have a systematic relationship in which properties are conceptual elements of their categories, which are, in turn, elements of the emerging theory. In terms of the present study, this necessitated examining each conversational sequence within the sample on the ground level as well as in relation to the context of the surrounding discourse. This allowed me to discover what conversational conditions evoked certain types of actions, and furthermore, the discursive consequences of said actions within subsequent conversation. 
Data and sampling. In order to qualitatively examine the nature of and potential social actions achieved by the opening sequences of cybergrooming conversation, I examined transcripts recorded and made public by the non-profit organization, Perverted Justice. Perverted Justice is a watchdog organization dedicated to identifying online sexual predators, publishing their identities online, and providing evidence against them to law enforcement. The group's strategy involves creating fictitious chat room handles and social network profiles that represent their adult decoys as minors, and cultivating a relationship through (often extensive) online dialogue with adults looking to talk about sex with the child. Then, the decoy agrees to arrange a meeting so as to expose the individuals' intention to actually meet in person to follow through on fantasies and hypothetical encounters discussed online. The decoys do not make first contact in chat rooms, but are known to be subsequently manipulative and aggressive once they identify a potential 'predator' (Hansen, 2007). Once a suspected predator is convicted, the entire chat log is published on the group's website (pervertedjustice.com).

These dialogues vary in length; some are relatively short and stem from only a few hours of chat, while others are extremely lengthy and can represent days or weeks of chat room discourse. More importantly, these transcripts represent an extremely rich source of data on the phenomenon of cybergrooming. Additionally they are naturallyoccurring; these conversations were not initiated by the researcher, nor did the researcher intervene in any way. As such, Perverted Justice chat transcripts offer significant breadth and depth of naturally-occurring cybergrooming behavior, and as they are publicly available in their entirety online, there was no risk of unnecessary harm to individuals or 
issues with confidentiality. The following transcript (author has added line numbers as

reference points) is part of one such document; it has been provided to exemplify the

nature of the data and will be referred to in the subsequent subsections:

(1) sahilmittal123 (03/07/08 9:10:16 PM): hi

(2) sahilmittal123 (03/07/08 9:10:18 PM): hw u doin

starringscarlett (03/07/08 9:10:25 PM): hi

starringscarlett (03/07/08 9:10:26 PM): kewl u

(3) sahilmittal123 (03/07/08 9:10:31 PM): good

(4) sahilmittal123 (03/07/08 9:10:34 PM): frm?

starringscarlett (03/07/08 9:10:55 PM): $13 \mathrm{f}$

starringscarlett (03/07/08 9:10:56 PM): u?

(5) sahilmittal123 (03/07/08 9:11:02 PM): 22m

(6) sahilmittal123 (03/07/08 9:11:10 PM): lik dating men?

starringscarlett (03/07/08 9:11:30 PM): yeah

(7) sahilmittal123 (03/07/08 9:11:46 PM): well then wud u lik to?

(8) sahilmittal123 (03/07/08 9:11:50 PM): am interested

(9) sahilmittal123 (03/07/08 9:11:56 PM): v can share pics 1st

starringscarlett (03/07/08 9:12:32 PM): yeah

(10) sahilmittal123 (03/07/08 9:12:34 PM): if i lik i might gve u some donation also

starringscarlett (03/07/08 9:12:39 PM): where u from?

(11) sahilmittal123 (03/07/08 9:12:57 PM): i am on telegraph road near intersection of $696 \mathrm{n} 10$

(12) sahilmittal123 (03/07/08 9:13:01 PM): $\mathrm{n} \mathrm{u}$ starringscarlett (03/07/08 9:13:19 PM): im in detroit

(13) sahilmittal123 (03/07/08 9:13:41 PM): k

(14) sahilmittal123 (03/07/08 9:13:50 PM): hw far $\mathrm{r} u$

(15) sahilmittal123 (03/07/08 9:13:51 PM): ?

(16) sahilmittal123 (03/07/08 9:14:05 PM): am new here i dnt knw the routes starringscarlett (03/07/08 9:14:18 PM): i dunno im 13 lol

starringscarlett (03/07/08 9:14:21 PM): i dont drive

(17) sahilmittal123 (03/07/08 9:14:24 PM): k

(18) sahilmittal123 (03/07/08 9:14:30 PM): home alone?

(19) sahilmittal123 (03/07/08 9:15:15 PM): b fast pls

starringscarlett (03/07/08 9:15:20 PM): yeah moms outta town

(20) sahilmittal123 (03/07/08 9:15:24 PM): k

Procedures. As the possible sample pool for this set of data was extremely large

(549 often lengthy transcripts), this study drew from it a manageable but representative

sample: At least the first 20 sequences of action from 100 transcripts; a sequence of 
action included one utterance by the cybergroomer as well as any contextually-relevant responses (see above example). I chose the opening sequences of discourse because in order to elicit what this phenomenon looks like for members, it was necessary to identify how this behavior manifests in its formative stages, as well as noting links between conditions and consequences that may be found in adjacent or nearby sequences of conversation. Additionally, I randomly sample these 100 transcripts from the sample pool by using a random number generator. The purpose behind this strategy was twofold. First, after initial categories began to emerge, this randomized approach maximized diversity within the sample. According to Glaser and Strauss (1967), such a strategy will stimulate the emergence of relevant properties and conditions: "His attempts to understand how these differences fit in are likely to have important effects on both his research operations and the generality of scope of his theory" (p.57). Second, it eliminated any potential researcher bias in selecting transcripts based on readily apparent features such as screen names and publishing dates, as well as bias that could have stemmed from the order and format in which they are presented on the website.

Units of analysis. This study's units of analysis were single utterances - or one time-stamped line of synchronous CMC discourse (see example above). This approach was appropriate in light of the CA framework that partially informed the study, which posits each speech act as influential as well as referential (Goody, 1978b). This strategy was especially useful in the present study, as the lack of any context not discussed during chat was unavailable, therefore obligating the analyst to be particularly sensitive to each aspect of every utterance (i.e. sequencing, positioning, and context, as well as what is actually said), and how these function within the microcosm of each conversation. 
However, as Goodwin and Heritage (1990) note, these discursive forms of action are both shaped by previous context and influence subsequent context. Therefore, while my units of analysis were single turns uttered by the cybergroomers, my coding and analysis could not properly capture their significance without considering salient utterances found earlier in chat. This process involved multiple levels of coding for each unit, which are outlined more specifically in the following subsection.

Analysis. The goal of the present research was to generate an inductive description of the concept of cybergrooming, focusing on the sequencing and social actions of discourse, that can be used as a building block toward a theory of this emergent phenomenon. Theory derived from grounded methods, according to Glaser and Strauss (1967), "is a strategy for handling data in research, providing modes of conceptualization for describing and explaining” (p. 3). I furthermore share these authors' view that such theory and description cannot be separated from the process that informs them: the inductive analysis of data. This process involves intensive coding procedures.

According to Strauss and Corbin (1998) analysis during grounded theory coding must be performed at two levels: the actual utterances in the sample and the researcher's conceptualization of them. These authors call for "microanalysis" of data, which is synonymous with detailed line-by-line analysis, "necessary at the beginning of a study to generate initial categories (with their properties and dimensions) and to suggest relationships among categories" (p. 57). This approach is generally utilized during open and axial coding and can apply to single words or whole utterances. This microscopic examination of phenomena was crucial in delineating the categories and conditions that manifested into themes, and thereby guided my theory-building process. My overall 
coding scheme followed the three-step process of open, axial and selective coding outlined by Strauss and Corbin. As my data was theoretically sampled, I did not look at these steps as a singular, linear process, but rather as an inverted funnel through which all of my data was be filtered as it was sampled.

Procedures. I first engaged in open coding, during which, “data are broken down into discrete parts, closely examined, and compared for similarities and differences" (Strauss \& Corbin, 1998, p. 102). For example, the first five lines in the above sample transcript all seem to involve greetings and basic age and location information, and were coded as such. Actions which were found to be conceptually linked began to form emergent concepts. Strauss and Corbin refer to these conceptual events as labeled phenomena, which allow them to be grouped with similar instances. Categories and subcategories then emerged as I grouped these phenomena into "more abstract high order concepts, based on [their] ability to explain what is going on" (p. 113). Categories were developed based on their properties and dimensions, and subcategories helped explain the phenomenon represented by a category. Here, properties are "the general or specific characteristics or attributes of a category," and dimensions refer to the "location of a property along a continuum or range" (p. 117). As such, in the above example, my coding scheme indicated that the aforementioned greetings and basic information dialogue occurred first and in continuous order, while the more personal inquiries in the following lines were coded individually as well as on the basis of their relative positioning. This allowed patterns to emerge as the links between the properties and dimensions of a category began to surface. In the present study, said properties involved both the social actions achieved by utterances and their relative sequencing/positioning within the 
context of discourse. During this phase of coding, phenomena were very often stamped with multiple codes, which were subsequently narrowed down and reformulated during the next phases.

The next step in the Strauss and Corbin (1998) scheme is axial coding, or finding links between categories and subcategories by considering the aforementioned properties and dimensions. More specifically, axial coding contextualizes emergent phenomena"to locate it within a conditional structure and identify the 'how' or the means through which a category is manifested" (p. 127). Another word for this is conditions, or the events that lead an individual to act in a certain way. As sequences of chat by the adult in these conversations served as the phenomena under scrutiny, axial coding addressed the discursive context for these potential conditions. For instance, lines six and seven in the above example were open-coded as separate actions but were combined during axial coding as they seemed, at surface level, to be getting at the same social action. The scope of this study was predominantly concerned with micro-level (as opposed to macro-level) conditions, as context extraneous to the data in the chat logs was largely unavailable. My interest was in discovering how cybergrooming themes manifest, function and potentially re-emerge conceptually throughout a dialogue. Thus, the micro-level context was especially important. Conditions can further be broken down into three types: causal, intervening, and contextual. Causal conditions are those that induce or influence the categorical phenomena, while intervening conditions mitigate the effect of these. Contextual conditions are "the specific sets of conditions that intersect dimensionally at this time and place to create the set of circumstances or problems to which persons respond through actions/interactions" (p. 132). Again, because my data did not include 
extraneous detail beyond what was said in conversation, contextual conditions were the most readily emergent, as well as significant, facets revealed during axial coding.

Finally, as I approached theoretical saturation, my analysis turned toward selective coding: "The process of integrating and refining the theory" (Strauss \& Corbin, 1998, p. 143). This final coding step is also dedicated to "density" or developing categories to their theoretical limits, which may involve further sampling and reversion to open and/or axial coding if additional concepts emerge. Coding continued until I reached theoretical saturation, or the point at which no new categories or properties were being discovered within the data (Glaser \& Strauss, 1967). Specific to the present study, this was the point at which I determined that no new themes were emerging out of the corpus of transcripts sampled, thus allowing me to conceptually fill the knowledge gat in the literature. As these coding stages required not only the careful description of categories, dimensions, and properties, but also constant comparison within and between these, I utilized Atlas.ti, software that facilitates analysis of large data samples.

Atlas.ti. Atlas.ti is qualitative data analysis software that allows for large bodies of data to be compiled and systematically sorted, which subsequently permits researchers to search for keywords and patterns, compare categories across data, and record memos for later reference (Darmody \& Byrne, 2006; Hwang, 2008; Lu \& Shulman, 2008). Darmody and Byrne (2006) found that the use of qualitative analysis software aids in theory building because of its ability to systematically sort through data, especially if the data set is very large. Lu and Shulman (2008) laud these types of programs for their facilitation of incorporating new themes and concepts as they emerge, while keeping the rest of the contextual data immediately accessible: "Exploratory coding schemes can 
evolve as soon as the first data is collected, providing an opportunity for a more thorough process, with consequent redirection if necessary” (p. 106). My study's grounded theory design called for exactly this type of continual analysis. Programs like Atlas.ti have also been praised for enhancing the transparency and validity of qualitative research (Hwang, 2008; Lu \& Shulman, 2008). Atlas.ti allowed me to systematically compile, access, and code my extensive data set. This was of great benefit to the present study as the sheer number of codes applied during analysis would have been challenging to manage without computer assistance. 


\section{Chapter 4: Results}

The following analysis presents themes that emerged from grounded-theoretic, open coding and subsequent generation of categories through grouping of open codes (see Methods). Because a 'theme' is defined as a "main subject that is being discussed or described" or "a particular subject or issue that is discussed often or repeatedly" (http://www.merriam-webster.com/dictionary/theme), it was operationalized as a category that occurred in at least $25 \%$ of all cases. The themes, listed in order of prevalence (see explanation of "prevalence" on next page), are: (1) conversational openings; (2) initial solicitation of age, sex and/or location; (3) specific questions regarding 'child's' life; (4) follow-up topicalization of 'child's' location; (5) seeking visual images of 'child;' (6) complimenting 'child's' appearance; (7) soliciting topic for discussion; (8) explicitly sexual statements; (9) soliciting 'child's' age preference for sex/romance; (10) arranging further contact; and (11) disclosing personal activities. The present section is broken down into subsections, each describing and discussing a single theme. Some themes are themselves comprised of subthemes, which are described and discussed in sub-sections. Three data fragments are provided as examples of each theme (or subtheme).

Regarding the presentation of each data fragment: (1) The full transcript from which it was drawn, and which can be found in Appendix A, is noted in the first line in brackets (e.g. "Extract 1 [Chat \#1]"); (2) Line numbers are presented, which correspond to those in the full transcripts (in Appendix A), and thus which give readers an idea of where the example is positioned relative to the roughly 38 lines of transcript that were coded for each chat (see below); (3) the male adult (i.e. the person who was ultimately 
arrested for attempting to groom a minor) is identified as 'MAN,' and the child decoy

(i.e. the adult member of Perverted Justice 'pretending' to be a child below the age of 17) is identified as 'DEC'; (4) the focal, coded unit of talk relevant to the theme (or subtheme) is presented in boldface type; and (5) coded units are separated by a paragraph (ף) symbol. In order to facilitate readability, timestamps have been omitted from data fragments, but have been retained in the full transcripts (Appendix A).

For each theme (or subtheme), a number of statistics are provided, including: (1) The prevalence of the theme (or subtheme) occurring at least once in any given chat, reported as a percentage, remembering that, by definition (see above) 'themes' had a prevalence of at least $25 \%$ (i.e. 'prevalence;' e.g., The theme occurs at least once in 25 chats out of 100); (2) The total number of times the theme occurs across all chats (i.e. 'total occurrence'); (3) The density of the theme, or the average number of times the theme occurs in chats when it occurs at least once (i.e. 'density;' e.g. When the theme occurs in a chat, it occurs an average of 2.2 times); (4) The statistical mean line number in which the theme occurs when it occurs at least once (i.e., 'positional mean'), which gives readers an idea of where the phenomenon tends to occur relative to the roughly 38 lines of transcript that were coded for each chat (see below); (5) the statistical range of line numbers in which the theme occurs when it occurs at least once (i.e., 'positional range'); and (6) the statistical standard deviation of line numbers in which the theme occurs when it occurs at least once (i.e., 'positional SD').

As noted in the methods section (see above), only the first 20 lines of the adult men's chat were analyzed and coded. Because chat interaction is, akin to ordinary conversation, sequenced (e.g. MEN's questions solicited DECOY's answers), chats 
averaged 37.77 lines in length $($ Range $=28-55 ; \mathrm{SD}=5.08)$. As such, particular line

numbers give readers a rough idea of where coded units of talk are positioned in chats.

For example, if a focal unit of talk occurs at line 19 , it occurs approximately $50 \%(37.7 \div$ $19=50.3 \%)$ into the analyzed chat, and thus around his tenth line of talk $(20 * .503=$ 10.06).

\section{Conversational Openings}

A larger-order theme emerged of 'conversational openings' (Schegloff, 1986), which was comprised of two sub-themes, including 'Greetings' and 'How-are-you's' (elaborated below). The prevalence of 'conversational openings' was $81 \%$ (total occurrence $=107 ;$ density $=1.32 ;$ positional mean $=2.14$; positional range $=1-12$; positional $\mathrm{SD}=1.83$ ). As expected, 'conversational openings' occurred extremely early, as virtual 'first moves' in chats. Greetings. The 'greetings' theme involved units of talk that were coded as accomplishing greeting actions (Schegloff, 1968; 1986), such as 'hey,' hi,' and 'hello.' The prevalence of 'greetings' was 75\% (total occurrence $=80$; density $=1.07$; positional mean $=1.58$; positional range $=1-7$; positional $\mathrm{SD}=1.09$ )

\section{Example 1 [Chat \#44]}

1 MAN (09/04/06 10:11:31 PM): hello there

2 DEC (09/04/06 10:11:38 PM): hey

3 DEC (09/04/06 10:11:39 PM): asl

\section{Example 2 [Chat \#31]}

1 MAN (07/18/06 12:28:36 PM): hey chick $\uparrow$ how are you

2 DEC (07/18/06 12:28:49 PM): hiya

3 MAN (07/18/06 12:29:12 PM): hows life treating you today

\section{Example 3 [Chat \#77]}

1 MAN (09/02/07 1:43:29 PM): hi

2 DEC (09/02/07 1:44:16 PM): hey

3 MAN (09/02/07 1:44:37 PM): hows it going tday 
Note that, in each case, the decoy orients to the man's greeting as such by responding back with a greeting, including "hey" (Example 1, line 2), "hiya" (Example 2, line 2), and "hey" (Example 3, line 2).

How are you. The 'how are you' theme involved units of talk that were coded as accomplishing personal-state inquiries (Sacks, 1975), including 'how are you,' 'how are you doing,' and 'how goes it.' The prevalence of 'how are you' was $27 \%$ (total occurrence $=27$; density $=1.00 ;$ positional mean $=3.81 ;$ positional range $=1-12$; positional $\mathrm{SD}=2.46)$

Example 4 [Chat \#8]

3 MAN (09/07/06 5:09:32 PM): hi

$4 \quad$ MAN (09/07/06 5:09:36 PM): how are u?

5 DEC (09/07/06 5:10:09 PM): ok as1

\section{Example 5 [Chat \# 9]}

1 DEC (11:58:40 PM): hi

2 MAN (11:58:47 PM): how goes it

3 DEC (11:58:57 PM): ok thanks how $\mathrm{r} u$ ?

\section{Example 6 [Chat \#99]}

2 DEC (03/08/08 6:56:36 PM): hi

3 MAN (03/08/08 6:57:02 PM): how you doin

$4 \quad$ MAN (03/08/08 6:57:53 PM): ?

$5 \quad$ DEC (03/08/08 6:58:16 PM): i am k u?

Note that, in each case, the decoy orients to the man's 'how are you' as a solicitation of a personal state by responding back with a 'bottom-line positive' evaluation (Pillet-Shore, 2011): “ok” (Example 4, line 5), “ok” (Example 5, line 3), and "I am k” (Example 6, line $5)$.

\section{Initial Solicitation of Age, Sex, and/or Location}

A larger-order theme emerged of 'initial solicitation of age, sex, and/or location,' which was comprised of one sub-theme, 'initial solicitation of ASL' (i.e. 
Age/Sex/Location), as well as three code categories that did not themselves constitute themes, per se, including 'initial solicitation of age,' 'initial solicitation of location,' and 'initial solicitation of sex' (soliciting sex, as its own action, was so rare that it did not warrant further pursuit as a standalone category). The justification for the validity of this larger-order theme (which admittedly includes three distinct topics) is that, in the context of online chat, the acronymal query "asl" (standing for "age, sex, and location") is extremely common, and thus, for chatters, the topics of age, sex, and location 'go together.' The prevalence of 'initial solicitation of age, sex, and/or location' was $73 \%$ (total occurrence $=75 ;$ density $=1.03 ;$ positional mean $=8.59 ;$ positional range $=1-29$; positional $\mathrm{SD}=6.37)$.

Initial solicitation of ASL. The 'initial solicitation of ASL' theme included units of talk which specifically solicited the 'ASL' (i.e. age, sex, and location) of the decoys. This was most commonly accomplished by either soliciting information with the acronym "ASL" or by responding to a decoy's asl-solicitation and then asking: "you?" (see Example 8, below). Alternatively, 'ASL' could be solicited by voluntarily disclosing such information as a 'first-positioned' interactional move (see Example 9, below). The prevalence of 'initial solicitation of ASL was $37 \%$ (total occurrence $=37$; density $=1.00$; positional mean $=5.76$; positional range $=1-20$; positional $\mathrm{SD}=3.29$ ).

\section{Example 7 [Chat \#47]}

\begin{tabular}{ll}
\hline 1 & MAN (09/09/06 6:28:26 PM): hi \\
2 & MAN (09/09/06 6:28:28 PM): asl? \\
3 & DEC (09/09/06 6:28:42 PM): heya whats up? \\
4 & DEC (09/09/06 6:28:47 PM): 13/f/socal whats ur asl?
\end{tabular}

\section{Example 8 [Chat \#7]}

3 DEC (11/11/06 7:50:59 PM): asl?

$4 \quad$ MAN (11/11/06 7:51:30 PM): 29/m/seattle 
5 MAN (11/11/06 7:51:32 PM): you?

$6 \quad \operatorname{DEC}(11 / 11 / 06$ 7:51:38 PM): $13 \mathrm{f}$ *Edited location*

\author{
Example 9 [Chat \#52] \\ 1 MAN (03/27/08 10:54:26 PM): hi \\ 2 MAN (03/27/08 10:54:32 PM): 28/male/nor cal \\ 3 MAN $(03 / 27 / 08$ 10:54:37 PM): how is it going? \\ 4 DEC (03/27/08 10:55:27 PM): hello 13/f/ca
}

Note that the decoy understands 'ASL' as a solicitation of 'age, sex, and location,' as when, in Example 7, the decoy responds with "13/f/socal" (i.e., 13 years old, female, from Southern California).

Initial solicitation of age. The 'initial solicitation of age' category involved units of talk that were coded as inquiring as to the age of the 'child' (i.e., decoy), such as "how old are you?" or "age?" The prevalence of 'initial solicitation of age' was 21\% (total occurrence $=21 ;$ density $=1.00 ;$ positional mean $=12.95 ;$ positional range $=1-29$; positional $\mathrm{SD}=8.50)$.

Example 10 [Chat \#65]

6 MAN (4:12:23 PM): how old are u ?

7 DEC (4:12:48 PM): 13/f/ca

8 MAN (4:13:26 PM): i'm 26 from LA

\title{
Example 11 [Chat \#14]
}

8 DEC (09/01/06 11:48:51 PM): how old $\mathrm{r} u$ ?

$9 \quad$ MAN (09/01/06 11:48:58 PM): $26:(27$ on tuesday

10 MAN (09/01/06 11:49:11 PM): u?

11 DEC (09/01/06 11:49:15 PM): wow happy birthday

12 DEC (09/01/06 11:49:17 PM): 13

\section{Example 12 [Chat \#67] \\ 3 DEC (9:13:10 AM): hi \\ 4 MAN (9:13:32 AM): age? \\ 5 DEC (9:13:47 AM): $13 \mathrm{u}$}

Note that, in each case, decoys understand these inquiries as ones for their age when they respond as such: "13/f/ca" (i.e., 13 years old, female, from California; Example 10, line 
7), "13" (Example 11, line 12), and "13” (Example 13, line 5). Insofar as decoys

sometimes respond to age-specific questions (e.g., "how old are u?"; Example 10, line 6) with age, sex, and location ("13/f/ca"; Example 10, line 7), there are grounds for including these inquiries into the larger theme of 'Initial Solicitation of Age, Sex, and/or Location.'

Initial solicitation of location. The 'initial solicitation of location' category involved units of talk that were coded as inquiring as to the location of the 'child' (i.e., decoy), such as "where are you from" and "where are you at," or ones that provided a location to be confirmed, such as "so are you in CO?" The prevalence of 'initial solicitation of age' was $17 \%$ (total occurrence $=17$; density $=1.00$; positional mean $=$ 9.35; positional range $=1-18$; positional $\mathrm{SD}=4.96$ ).

Example 13 [Chat \#63]

15 DEC (09/08/06 9:45:32 PM): im not im sorry

16 MAN (09/08/06 9:45:43 PM): where are u from

17 DEC (09/08/06 9:45:55 PM): cali

Example 14 [Chat \#77]

6 DEC (09/02/07 1:45:54 PM): yea me to

$7 \quad \operatorname{MAN}(09 / 02 / 07$ 1:46:03 PM): were you at

8 DEC (09/02/07 1:46:21 PM): ga

Example 15 [Chat \#9]

11 DEC (12:00:01 AM): lol

12 MAN (12:00:18 AM): so are you in CO?

13 DEC (12:00:37 AM): yea i jus now moved here

Note that, in each case, decoys understand these inquiries as ones for their geographic location when they respond as such: "cali" (i.e., California; Example 13, line 17) and "ga" (i.e., Georgia; Example 14, line 8). 


\section{Specific Questions Regarding Child's Life}

The theme 'specific questions regarding child's life' generally emerged later in chat than 'solicit topic for discussion,' and featured inquiries by the adults for more specific and personal (non-sexual) updates about the 'children.' This category has a diverse set of examples and can range from asking about the 'child's' school schedule, living situation, personal qualities/beliefs, etc., to preferred (non-sexual) activities, hobbies, and aspirations. The prevalence of 'specific questions regarding child's life' was $55 \%($ total occurrence $=110 ;$ density $=2 ;$ positional mean $=25.14 ;$ positional range $=1-$

47; positional $\mathrm{SD}=9.92$ ).

\section{Example 16 [Chat \# 34]}

23 DEC (6:07:10 PM): ya

24 MAN (6:08:10 PM): so u still in school

25 DEC (6:08:15 PM): ya

26 MAN (6:08:28 PM): cool 9 wat grade

27 DEC (6:09:20 PM): $8^{\text {th }}$

\section{Example 17 [Chat \#5]}

14 DEC (10/11/08 9:24:09 PM): like western part

15 MAN (10/11/08 9:24:24 PM): what are ur hobbies

16 DEC (10/11/08 9:24:47 PM): hanging out watching tv cheerleading

\section{Example 18 [Chat \#83]}

29 DEC (2:31:01 PM): kewl. i dont relly kno alot about tx

30 MAN (2:31:11 PM): who do you live with

31 DEC (2:31:16 PM): my dad

\section{Follow-up Topicalization of 'Child's' Location}

A theme emerged of 'follow-up topicalization of child's location.' This theme represents cases in which, after already having been informed of the decoys' location which was almost always a product of decoys having answered 'initial' inquiries regarding their location (see above) - men continued to topicalize the decoys' location, 
such as requesting more specific locational information, such as city or region. The prevalence of 'follow-up topicalization of child's location' was $44 \%$ (total occurrence = 73 ; density $=1.66$; positional mean $=17.12$; positional range $=2-41$; positional $\mathrm{SD}=$

\section{$8.54)$.}

\section{Example 19 [Chat \#65]}

6 MAN (4:12:23 PM): how old are u ?

$7 \quad$ DEC (4:12:48 PM): 13/f/ca

8 MAN (4:13:26 PM): i'm 26 from LA

$9 \quad$ MAN (4:13:36 PM): where in cali ?

10 DEC (4:13:38 PM): riverside

\section{Example 20 [Chat \#8]}

9 MAN (09/07/06 5:12:16 PM): where do u live

10 DEC (09/07/06 5:13:25 PM): 12 f cali

11 MAN (09/07/06 5:13:33 PM): where in ca

12 DEC $(09 / 07 / 06$ 5:13:49 PM): socal

13 MAN (09/07/06 5:14:01 PM): i live near Disneyland

Example 21 [Chat \#75]

3 DEC (10/03/08 8:28:17 PM): hi there, asl?

$4 \quad$ MAN (10/03/08 8:28:23 PM): hi

$5 \quad$ MAN (10/03/08 8:28:36 PM): 28/m/hillsdale u?

$6 \quad$ DEC (10/03/08 8:28:44 PM): 14f/mi

$7 \quad$ MAN (10/03/08 8:29:03 PM): cool

$8 \quad$ MAN (10/03/08 8:29:08 PM): what part ?

$9 \quad \operatorname{DEC}(10 / 03 / 08$ 8:29:14 PM): west $u$ ?

10 MAN (10/03/08 8:29:43 PM): lol im in hillsdale at the bottom of the state

\section{Seeking Visual Image of 'Child'}

A relatively robust theme involved utterances in which men requested visual

images of 'children' (i.e., decoys). This theme was relatively dense, meaning that, when it occurred, it occurred multiple times. Most commonly, this involved asking the 'children' for photographs of themselves, but also included rare instances where men simply requested physical descriptions of the 'children.' The prevalence of 'seek visual 
CYBERGROOMING

image of "child" was $40 \%$ (total occurrence $=72$; density $=1.85$; positional mean $=$

22.49; positional range $=2-41$; positional $\mathrm{SD}=9.46$ ).

Example 22 [Chat \#82]

17 MAN (7:05:01 PM): OK

18 MAN (7:05:07 PM): U HAVE ANY PICS

19 DEC (7:05:13 PM): ya do u

20 MAN (7:05:37 PM): YEAH ON PROFILE $\uparrow$ CAN U SEND TO MY EMAIL

21 DEC (7:06:16 PM): yeah can u send pix 2 my email i cant get ur prof open

Example 23 [Chat \#100]

27 DEC (8:39:15 PM): lol asl

28 MAN (8:39:25 PM): u got cam or more pics

29 DEC (8:39:32 PM): nah

Example 24 [Chat \#30]

17 MAN (01/21/08 2:06:43 AM): its sucks

18 DEC (01/21/08 2:06:50 AM): oh

19 MAN (01/21/08 2:07:05 AM): pics?

20 DEC $(01 / 21 / 08$ 2:07:46 AM): yea

21 DEC (01/21/08 2:07:49 AM): you?

22 MAN (01/21/08 2:08:08 AM): getting those on my computer also here in a few

23 MAN (01/21/08 2:08:11 AM): can i see ur's?

24 DEC (01/21/08 2:08:21 AM): ok

$25 \operatorname{MAN}(01 / 21 / 08$ 2:09:29 AM): wow ur hott for a 13 yr old

26 DEC (01/21/08 2:09:38 AM): thanks

\section{Complimenting 'Child's' Appearance}

A theme emerged regarding men complimenting children (i.e., decoys) on their physical appearance. Note that, in some (perhaps many cases), decoys' pictures are available on their profile pages, and thus available to men even prior to chatting. The prevalence of 'compliment appearance' was $39 \%$ (total occurrence $=69$; density $=1.77$;

positional mean $=18.48$; positional range $=1-40$; positional $\mathrm{SD}=11.79$ )

Example 25 [Chat \#30]

23 MAN (01/21/08 2:08:11 AM): can i see ur's?

24 DEC (01/21/08 2:08:21 AM): ok

25 MAN (01/21/08 2:09:29 AM): wow 9 ur hott for a 13 yr old

26 DEC (01/21/08 2:09:38 AM): thanks 


\section{Example 26 [Chat \#64]}

$35 \quad$ MAN (03/27/07 4:40:37 PM): oh,i shouldve known

36 DEC (03/27/07 4:40:43 PM): lol

37 MAN (03/27/07 4:41:18 PM): you look like a beauty queen

38 DEC (03/27/07 4:41:35 PM): awe

39 DEC (03/27/07 4:41:39 PM): ur sweet

\section{Example 27 [Chat \#15]}

$4 \quad$ MAN (9:13:28 PM): I feel dirty

$5 \quad \operatorname{DEC}(9: 13: 36 \mathrm{PM}):$ y lol?

$6 \quad \operatorname{MAN}(9: 13: 43 \mathrm{PM}):$ your a hottie

$7 \quad \operatorname{DEC}(9: 14: 25 \mathrm{PM}):$ tyvm

Note that the decoys understand these compliments as such, and commonly orient to them in the 'preferred' manner (Goodwin \& Heritage, 1990; Sidnell, 2010), by acknowledgment and 'thanking' the adult, as when, in Example 25 (line 26), the decoy responds with "thanks," and in Example 27 (line 7), the decoy responds with "tyvm" (i.e. thank you very much), and/or by replying with their own compliment, as when, in Example 26 (line 39), the decoy responds with "ur sweet." 'Compliments,' as a theme, is thus a strong example of successful social actions initiated by the men. The often sexualized language used by the men performing these actions, as well as the fact that the category includes only compliments regarding physical appearance (as opposed to personality traits, etc.), justifies its classification as adult-child sexual communication.

\section{Soliciting Topic for Discussion}

The theme 'solicit topic for discussion' involved utterances akin to Button \& Casey's (1985) topic-initial solicitations, where men solicited, in very non-specific and open-ended ways, topics for discussion from children, such as: "What's up?", "What are you doing?", "How's life treating you?", "How was your day?", and "What's going on 
tonight?" The prevalence of 'solicit topic for discussion' was 36\% (total occurrence $=$ $43 ;$ density $=1.19 ;$ positional mean $=11.56$; positional range $=2-41 ;$ positional $\mathrm{SD}=$ 9.68).

Example 28 [Chat \#95]

$7 \quad$ MAN (2:12:41 PM): oh

8 MAN (2:13:19 PM): hows your day

9 DEC (2:13:30 PM): boring lol

10 MAN (2:13:36 PM): i'm sorry to hear that

Example 29[Chat $\# 57$

9 MAN (03/12/07 3:20:08 PM): cool me too

10 MAN (03/12/07 3:20:29 PM): what are you doing today

11 DEC (03/12/07 3:20:47 PM): chatin

Example 30 [Chat \#14]

1 MAN (09/01/06 11:47:15 PM): hi ;;)

2 DEC (09/01/06 11:47:22 PM): hi

$3 \quad$ MAN (09/01/06 11:47:28 PM): whats up

$4 \quad \operatorname{DEC}(09 / 01 / 06$ 11:47:38 PM): nuthin

Note again that in most of these cases, the decoy understands these inquiries as such and responded to them in the 'preferred' manner, as when, in Example 28 (line 9), the decoy responds with "boring lol," and in Example 30 (line 4), "nuthin."

\section{Explicitly Sexual Statements}

A larger-order theme emerged of men making explicitly sexual comments or inquiries. This theme is comprised of the sub-theme of 'sexual questions,' and the smaller sub-categories of 'sexual self-disclosure,' 'sexual statement,' and 'discussion/description of sex act.' The prevalence of 'explicitly sexual statement' was 35\% (total occurrence $=$ $95 ;$ density $=2.71 ;$ positional mean $=27.08 ;$ positional range $=2-50 ;$ positional $\mathrm{SD}=$ $10.33)$. 
Sexual question. Through linguistic design - that is, through interrogative

syntax, such as a Yes/No-question or a Wh-question - 'sexual questions' solicited

information about explicitly sexual topics. The prevalence of ‘sexual question' was $26 \%$

(total occurrence $=68 ;$ density $=2.61 ;$ positional mean $=27.45 ;$ positional range $=2-50$;

positional $\mathrm{SD}=10.10)$

Example 31 [Chat \#1]

26 MAN (11:07:33 PM): ty

27 MAN (11:08:23 PM): ever have a guy masterbate for $u$ in person

28 DEC (11:08:36 PM): no

29 MAN (11:08:50 PM): what all have u done

30 DEC (11:09:05 PM): had sex

Example 32 [Chat \#18]

16 DEC [11:56 PM]: and i dont wanna run into some1 i kno

17 MAN [11:57 PM]: haha ok... ๆ so how big is your dick?

18 DEC [11:57 PM]: like 5"

19 DEC [11:57 PM]: urs?

\section{Example 33 [Chat \#15]}

25 DEC (9:18:53 PM): oh okay

$26 \operatorname{MAN}(9: 19: 30 \mathrm{PM})$ : so are you a virgin?

27 DEC (9:19:39 PM): no

28 MAN (9:19:54 PM): wow, I like you

Sexual self disclosure. Men self-disclosing sexual details about themselves

formed a category (i.e., It was not prevalent enough to constitute a theme, as such). Such statements took the form of self-disclosed sexual history, sexual desires or descriptions of sex acts the men have performed or would like to in the future. The prevalence of 'sexual self disclosure' was $12 \%$ (total occurrence $=18$; density $=1.5$; positional mean $=27.33$;

positional range $=4-46$; positional $\mathrm{SD}=10.62$ )

Example 34 [Chat \#19]

26 MAN (10/03/08 9:33:46 PM): what the oldest guy uv ever been with

27 DEC (10/03/08 9:34:16 PM): my ex jason 15

28 MAN (10/03/08 9:34:27 PM): wow thats really old 
29 DEC (10/03/08 9:34:36 PM): yeah

30 MAN (10/03/08 9:34:48 PM): the youngest girl ive ever been with was 16 when i was 20

31 DEC (10/03/08 9:35:08 PM): oh ok

32 MAN (10/03/08 9:35:36 PM): its funny cause i keep thinking back to that saying if theres grass on the field play ball

Example 35 [Chat \#4]

27 MAN (07/21/09 4:20:03 PM): would yo ulike me to rub your back

28 DEC (07/21/09 4:20:24 PM): might b kool

29 MAN (07/21/09 4:20:42 PM): i would rub your butt softly fo ryou

30 DEC (07/21/09 4:21:04 PM): really?

31 MAN (07/21/09 4:21:15 PM): would you like that

32 DEC (07/21/09 4:21:21 PM): idk

33 DEC (07/21/09 4:21:26 PM): might b ok

34 MAN (07/21/09 4:21:53 PM): i would pu tmy hand insid eyour panties when i rub it

35 DEC (07/21/09 4:22:07 PM): $\mathrm{k}$

36 MAN (07/21/09 4:22:30 PM): how about your slit?

37 DEC (07/21/09 4:23:45 PM): idk

38 MAN (07/21/09 4:24:10 PM): i just use my tongue on that

39 DEC (07/21/09 4:24:21 PM): really?

$40 \quad \operatorname{MAN}(07 / 21 / 09$ 4:24:28 PM): yes

\section{Example 36 [Chat \#78]}

14 MAN [12:50 PM]: yes, of course i am

15 DEC [12:50 PM]: thats so gay

16 MAN [12:51 PM]: why, ๆ you should see some of those bois naked bodies, waht a turn on ,,its hard to not want them

17 DEC [12:51 PM]: they dont got hair

18 MAN [12:52 PM]: well most dont, and there the ons i like to "watch"

Sexual statement. Another category involved men's sexual statements that were neither questions nor self disclosures (Again, this category was not prevalent enough to constitute a theme, per se). The prevalence of 'sexual statement' was 7\% (total occurrence $=8 ;$ density $=1.14 ;$ positional mean $=24.12 ;$ positional range $=10-40$;

positional $\mathrm{SD}=11.45)$

Example 37 [Chat \#67]

10 MAN (9:14:27 AM): unless you dont mind 
CYBERGROOMING

11 MAN (9:14:55 AM): i wont talk about sex because you a virgin and never saw a cock

12 DEC (9:15:02 AM): ok

Example 38 [Chat \#62]

29 MAN (07/20/06 7:09:21 PM): well how do i know u r sweet w/o tasting? lol

30 DEC (07/20/06 7:09:34 PM): i don't know really

31 DEC (07/20/06 7:09:43 PM): i guess i don't

32 DEC (07/20/06 7:09:46 PM): :(

33 MAN (07/20/06 7:10:01 PM): awwww i guess i'll just have to try it

34 DEC (07/20/06 7:10:41 PM): whatcha thinking?

35 MAN (07/20/06 7:10:52 PM): about us

Example 39 [Chat \#18]

17 MAN [11:57 PM]: haha ok... so how big is your dick?

18 DEC [11:57 PM]: like 5"

19 DEC [11:57 PM]: urs?

20 MAN [11:57 PM]: lol.. ๆ u had your first sexy gay chat!!!! ๆ HAHA

21 MAN [11:57 PM]: 7

22 DEC [11:57 PM]: lol

23 DEC [11:57 PM]: well not my 1st lol but thanx :)

Note the progression of sexual chat in the above examples. The men in these chats

commonly introduced sexualized topics via flirtatious or 'playful' statements or questions, and followed these with more direct, specified or explicit sexual statements, as when, in Example 38 (line 29), the man asks "how do I know u r sweet w/o tasting?", and follows with the more direct statement (line 33), "I guess I'll just have to try it," and in example 39, the man asks a sexual question (line 17) and immediately encourages the pattern of sexualized chat (line 20).

\section{Seeking 'Child's' Age Preference for Sex/Romance}

A theme emerged of men seeking the 'children's' (i.e., decoys') age preferences regarding sexual encounters and/or romantic relationships. This theme included direct strategies, such as men explicitly asking for children's age preferences, as well as more indirect strategies, such as men asking decoys if they liked 'older men,' or men seeking 
children's approval/appraisal of men's self-disclosed age. The prevalence of 'seek age

preference' was $35 \%$ (total occurrence $=50$; density $=1.43$; positional mean $=20.60$;

positional range $=5-40$; positional $\mathrm{SD}=9.83$ ).

Example $40[$ Chat \#70]

$35 \quad$ MAN (03/23/07 1:17:11 PM): :)

36 MAN (03/23/07 1:17:43 PM): so what would u say your limit is as far as age you want your bf to be?

37 DEC (03/23/07 1:18:08 PM): i duno i like older but not like $90 \mathrm{n}$ all rinkly $\mathrm{n}$ shit lol

38 MAN (03/23/07 1:18:31 PM): lol

\section{Example 41 [Chat \#4]}

13 DEC (07/21/09 4:13:28 PM): like might tell $\mathrm{u}$ when i know u better

14 MAN (07/21/09 4:13:45 PM): ok, ๆ you like older men heather

15 DEC (07/21/09 4:14:08 PM): ya

16 DEC (07/21/09 4:14:12 PM): they r ok

17 MAN (07/21/09 4:14:28 PM): what do you like them to do

Example 42 [Chat \#98]

12 DEC (07/18/06 9:44:20 PM): wow thats cool

13 MAN (07/18/06 9:44:36 PM): so can i ask, 9 am i to old for you to talk to and all?

14 DEC (07/18/06 9:44:45 PM): lol no your not a grandpa

15 MAN (07/18/06 9:44:54 PM): i know thats right lol

\section{Arrange Further Contact}

A theme emerged of arranging further contact with the 'children' (i.e., decoys).

This theme represents attempts by the adults to pursue contact with the decoys outside of the chat. The theme of 'arrange further contact' is made up of two open code categories that were not themselves prevalent enough to constitute themes, per se: 'arrange meeting' and 'arrange other contact,' where the former includes attempts to convince the decoys to meet in person, and the latter includes attempts to contact the children via another medium (e.g. phone, e-mail, social networks). The prevalence of 'arrange further contact' 
was $28 \%$ (total occurrence $=50$; density $=1.78$; positional mean $=24.44$; positional range $=8-46$; positional $\mathrm{SD}=9.31$ )

Arrange meeting. The 'arrange meeting' category involved attempts by men to set up offline, in-person meetings with decoys. Such attempts involved a range of implicit to explicit requests, such as "interested in meeting?", "maybe sometime we could do lunch," and "this would be much more fun in person." The prevalence of "arrange meeting' was $18 \%($ total occurrence $=31$; density $=1.72$; positional mean $=23.71$; positional range $=8-46$; positional $\mathrm{SD}=9.49$ ).

Example 43 [Chat \#21]

6 MAN (3:04:16 PM): have more pictures of you? I have a few

7 DEC (3:07:09 PM): yeah i have a couple somewehere

8 MAN (3:07:34 PM): interested in meeting?

9 DEC (3:07:55 PM): maybe. u cute lol

Example 44 [Chat \#76]

22 MAN (03/02/08 2:39:14 PM): thanks

23 MAN (03/02/08 2:40:30 PM): mabey sometime we could do lunch

$24 \quad$ MAN (03/02/08 2:40:39 PM): ;)

25 DEC (03/02/08 2:41:34 PM): that be kewl

\section{Example 45 [Chat \#1]}

20 MAN (11:06:27 PM): do u want me to cum yet

21 DEC (11:07:18 PM): i dunno

22 MAN (11:07:33 PM):

23 MAN (11:08:04 PM): this would be much funner doing this for $\mathbf{u}$ in person

24 DEC (11:08:14 PM): oh

Arrange other contact. The 'arrange other contact' category represents attempts by men to communicate with 'children' (i.e., decoys) via other means, which generally involved more immediate and/or personal mediums, such as telephone, email, and social networks. The prevalence of 'arrange other contact' was $12 \%$ (total occurrence $=19$; density $=1.58$; positional mean $=25.63$; positional range $=13-41$; positional $\mathrm{SD}=8.89$ ) 
Example 46 [Chat \#43]

13 MAN (5:43:52 PM): 16/m/north hollywood

14 MAN (5:43:53 PM): is that ok?

15 MAN (5:44:47 PM): want to talk on the phone?

16 DEC (5:44:55 PM): dood im lookin 4 older

Example 47 [Chat \#24]

20 DEC k

21 MAN what's ur url on myspace though?

22 DEC *my myspace url*10

23 MAN you're hot

Example 48 [Chat \#73]

39 MAN (9:39:16 PM): do u have sell ?

40 DEC (9:39:21 PM): huh

41 MAN (9:39:36 PM): give me ur nemember

42 DEC (9:39:46 PM): my wha

43 MAN (9:40:29 PM): ur phone numemebr

44 DEC (9:40:39 PM): no way dude my mom is home

\section{Disclosing Personal, Non-Sexual Activities}

A theme emerged involves utterances in which men self disclose their own

present or past non-sexual activities. The prevalence of 'disclose personal, non-sexual

activity' was $25 \%$ (total occurrence $=32$; density $=1.28$; positional mean $=19.30$;

positional range $=1-41$; positional $\mathrm{SD}=10.03$ )

Example 49 [Chat \#94]

25 DEC (09/24/08 10:20:33 PM): well noone ever took me before they ;r all too busy i guess

26 MAN (09/24/08 10:21:30 PM): that is to bad 1 i work a 47.5 hours aweek at my job and farm and still find time to go camping

27 DEC (09/24/08 10:21:39 PM): wow

Example 50 [Chat \#31]

$5 \quad$ MAN (07/18/06 12:30:30 PM): i cant complain

$6 \operatorname{MAN}(07 / 18 / 06$ 12:30:43 PM): taking a break from the heat right now .. been washing a camper

$7 \quad \operatorname{MAN}(07 / 18 / 06$ 12:32:16 PM): so what are you up to this summer

\footnotetext{
${ }^{10}$ URL's, e-mail addresses and other specific information are often removed by the decoy prior to publication of transcript.
} 
Example 51 [Chat \#49]

14 DEC (9:26:02 PM): i am 14 i cant go 2 them kinds of partys yet

15 DEC (9:26:10 PM): mom wont let me

$16 \operatorname{MAN}(9: 26: 38 \mathrm{PM})$ : when i was 14, i was expelled for the sec time from junior high

17 DEC (9:26:47 PM): wow hehe y?

18 MAN (9:27:11 PM): caught with pot on school grounds

Notable Non-robust Categories

Some categories that emerged either during open or axial coding did were not prevalent enough to constitute themes, per se (i.e., because they occurred in less than $25 \%$ of cases), yet seemed thematically relevant. Therefore, this section will briefly introduce the following non-robust categories and subcategories: 'age pursuit,' 'acknowledge inappropriateness' and 'isolation inquiry.'

Pursuing 'Child's' Age. While 'follow-up topicalization of 'child's' location' was a very robust theme, an 'age pursuit' category also emerged. These actions involve various discursive methods of confirming the age reported or posted to a profile page by the decoy (such pre-chat information seems to account for 'age pursuit' occurring as early as line 1) or commenting that the decoy seems to be older than originally stated.

The prevalence of 'age pursuit' was $22 \%$ (total occurrence $=29$; density $=1.32$;

positional mean $=10.93$; positional range $=1-38$; positional $\mathrm{SD}=9.94)$.

\section{Example 52 [Chat \#11]}

5 DEC (08/22/06 12:16:12 AM): 13/f/ca, how $\mathrm{r}$ u?

$6 \quad$ MAN $(08 / 22 / 06$ 12:16:18 AM): 13?

$7 \quad$ MAN (08/22/06 12:16:20 AM): wow

$8 \quad$ MAN (08/22/06 12:16:21 AM): really?

$9 \operatorname{DEC}(08 / 22 / 06$ 12:16:27 AM): ya

Example 53 [Chat \#13]

1 MAN (07/20/06 5:52:18 PM): sassy how old are you really

$2 \quad$ MAN $(07 / 20 / 06$ 5:52:28 PM): 13 or 14

$3 \quad \operatorname{DEC}(07 / 20 / 06$ 5:53:07 PM): 13 why? 
4 MAN (07/20/06 5:53:27 PM): that is what $\mathrm{i}$ thought...yahoo has you marked as 113

Example 54 [Chat \#38]

1 MAN (04/16/08 11:22:17 PM): so you are only 14?

2 DEC (04/16/08 11:22:28 PM): yah

3 DEC (04/16/08 11:22:32 PM): so wut

Acknowledging wrongdoing. Another notable category emerged from the men

discursively commenting or acknowledging the moral problems involved in chatting with a young teenager. Though 'acknowledge inappropriateness' did not qualify as a theme ( $25 \%$ prevalence), it should be noted that many of the men obviously knew what they were doing would be viewed as immoral. Many cases within this category involved 'wishing' the 'child' was of legal age or otherwise discussing the 'wrongness' of their own actions. The prevalence of 'acknowledge inappropriateness' was 18\% (total occurrence $=21 ;$ density $=1.17 ;$ positional mean $=21.81 ;$ positional range $=8-37$; positional $\mathrm{SD}=9.42$ )

Example 55 [Chat \#19]

32 MAN (10/03/08 9:35:36 PM): its funny cause i keep thinking back to that saying if theres grass on the field play ball

33 MAN (10/03/08 9:35:51 PM): but 14 is way too young

34 DEC (10/03/08 9:35:52 PM): whats that mean

35 MAN (10/03/08 9:36:05 PM): wow u really are young

Example 56 [Chat \#68]

31 MAN (03/25/07 5:47:57 PM): well take care....nice chattin

32 DEC (03/25/07 5:49:44 PM): u goin?

33 MAN (03/25/07 5:50:01 PM): well prob shouldnt be talkin to ya

34 MAN (03/25/07 5:50:34 PM): what do ya like to do for fun????

35 DEC (03/25/07 5:50:53 PM): mostly hang out wit freinds $n$ watevers fun

Example 57 [Chat \#15]

13 DEC (9:15:19 PM):;;)

14 MAN (9:15:24 PM): still wish you were 18

15 MAN (9:16:05 PM): so how many older guys try to pick you up?

16 DEC (9:16:46 PM): not too many 
Asking if 'child' is alone. Finally, an interesting category, given the context of

this research, emerged from specified inquiries made about if and/or when the youth will be home alone. Though the category 'isolation inquiry' did not occur in $25 \%$ of chats, it is nonetheless an interesting topic to consider given the theme of this research. The prevalence of 'isolation inquiry' was $8 \%$ (total occurrence $=10$; density $=1.25$;

positional mean $=30.3$; positional range $=17-45$; positional $\mathrm{SD}=9.13$ ).

Example 58 [Chat \#3]

18 DEC (03/31/07 4:41:45 PM): i cut a few days cos my mom went to vegas

19 DEC (03/31/07 4:41:46 PM): ;)

$20 \quad$ MAN (03/31/07 4:41:50 PM): nice

$21 \operatorname{MAN}(03 / 31 / 07$ 4:42:02 PM): and ur home alone?

22 DEC (03/31/07 4:42:09 PM): yeah

Example 59 [Chat \#14]

23 DEC (09/01/06 11:52:30 PM): i dont know wut u wana do?

$24 \operatorname{MAN}(09 / 01 / 06$ 11:52:39 PM): where your parents at?

25 DEC (09/01/06 11:52:52 PM): my moms downstairs and my dad is dead

26 MAN (09/01/06 11:53:02 PM): oh >:D< sorry to hear that

Example 60 [Chat \#23]

38 DEC (3:53:33 PM): ya

39 MAN (3:53:48 PM): So are you by yourself all day?

40 DEC (3:54:20 PM): no 


\section{Chapter 5: Discussion}

The analytical results detailed above present a unique window into how cybergrooming discursively manifests itself, how language is structured, ordered, and expressed by those who perform the behavior, and what social actions these men perform while communicating in chat rooms. The data, drawn from the first 20 lines of each of 100 chats, represent a wide and diverse sample of this communication, the scope of which has not been approached in any of the topic's research corpus. As such, the following discussion touches upon an extremely varied set of discursive actions performed with a wide range of sequencing and organization. Nonetheless, all 11 themes emerged with enough regularity and patterning to begin to paint a picture of what the initiation of cybergrooming is most apt to looks like. In practical terms, the ability to recognize the early signs of predatory behavior is an important tool for educational, law enforcement, and public safety purposes. The following discussion will explicate each theme by presenting it in the context of the present data, exploring how it may or may not align with prior research, and suggesting how it might further the scope of knowledge on the subject. The subsections herein are organized on a rough continuum, beginning with features the literature indicates are likely to be found in 'normal,' non-predatory chat dialogue, and progressing toward features that more clearly mark discourse as sexualized or otherwise inappropriate for an adult-child interaction.

\section{Opening Up Conversations}

$\mathrm{CMC}$ has characteristics in common with both spoken and written language but tends to more closely resemble spoken communication (Herring, 1999; Zhou, 2012). Thus, like any interpersonal interaction, synchronous online chat is oriented to by 
participants as a conversational action and must necessarily begin with mutuallyrecognized contact initiation (Gonzalez-Lloret, 2011; Herring, 1999; Markman, 2009; Rintel, Mulholland, \& Pittam, 2001; Silverman, 1998; Simpson, 2005; Tang, 2007; Wachs, Wolf \& Pan, 2012). The data for this study reflected this norm, as $81 \%$ of the men engaged in some form of conversational opening (conceptualized as 'greetings', such as "hello," "hi," and "hey," and 'how are you' questions).

Greetings. In face-to-face interactions, greetings are typically expected and reciprocated (Silverman, 1998), and are described as minimal adjacency pairs (Schegloff, 2007), or ones which are constituted by 2 turns and are generally adjacent (Curl \& Drew, 2008; Sacks et al., 1974; Schegloff, 1968; Sidnell, 2010; Zhou, 2012). A majority (75\%) of the men in these chats adhered to such expectations by either initiating or reciprocating a greeting statement, showing that while the norms of conversation may be diminished in many aspects of CMC, greeting the other party is still commonly utilized to invoke the beginnings of social cohesion (Goodwin \& Heritage, 1990; Pomerantz, 1984; Zhou, 2012) as well as mutually establishing that a conversation has started (Tang, 2007). Expected sequencing norms also held true in most cases; the typical greeting occurred within the first 2 lines of chat (positional mean $=$ line 1.58). This did not vary significantly across the sample (positional SD $=1.09$ ), and never occurred beyond line 7 .

'How are you?' The 'how are you' sub-theme fell under the theme of 'conversational openings.' Such statements partially align with Sacks (1975), who points out the prevalence of personal-state inquiries at the start of face-to-face conversations, as well as with Zhou (2012) who observed a similar pattern in CMC. While the prevalence (27\%) of the 'how are you' category was much lower than that of simple greetings, these 
inquiries generally reflected expected norms when they did emerge. Gonzalez-Lloret (2011) noted that while the overall structure of a CMC interaction often does not reflect the rules of sequence organization and turn-taking one might expect from face-to-face conversations, it does feature "individual strands or conversations which do seem to adhere" to these basic rules (p. 311). This was predominantly true when 'how are you' occurred in the openings that began the chats within the sample.

Theoretical implications. The prevalence of greetings in the present study's data (75\%) is well over Zhou's (2012) finding that $45 \%$ of MSN chats opened with greeting exchanges. This is further evidence that conversational openings, overall, were an area in which typical conversational norms were most notably adhered to; greetings were often met with similar statements, and 'how are you' questions were very often reciprocated. While it is now well-established that the adjacency pair system (Curl \& Drew, 2008; Sacks et al., 1974; Schegloff, 1968; Sidnell, 2010) breaks down significantly in CMC (Gonzalez-Lloret, 2011; Hancock \& Dunham, 2010; Markman, 2009; Subrahmanyam et al., 2004; Walther, 1996; Zhou, 2012), its lingering effects on human communication can be most readily observed in these early and relatively standardized parts of a chat dialogue. However, this is not to say that traditional turn-taking rules, or even normalized conversational openings themselves, are ubiquitous in the beginnings of chats; some of the subjects did indeed skip this step entirely. Nor can one expect to find traditionally sequential adjacency pairs in any sort of regular pattern in synchronous CMC; in fact this theory could be furthered by conceptualizing CMC adjacency pairs as not necessarily adjacent but neighboring, as $\mathrm{CMC}$ is typically too fast-paced for one party to wait for a response before speaking again. 
Conversational openings in cybergrooming. In terms of sexual luring and grooming, greetings are also expected features of the process, whether perpetrated offline (Lang \& Frenzel, 1988; Malesky, 2007; Olson et al., 2007) or online (O’Connell, 2004; Whittle et al., 2013). Olson et al. (2007) noted that luring generally begins with the causal condition of gaining access, and Lang and Frenzel (1988) similarly note that offenders who target children usually try to isolate the potential victim before attempting any crime. The present research suggests that access and isolation are both virtually guaranteed in an online setting; therefore these safeguards are not prescient shields against cybergrooming. Thus the concept of "strategic placement" (Olson et al., 2007, p. 239) in CMC boils down to little more than choosing the right Internet location (i.e. chat room) that youth frequent. This echoes Gottschalk (2011), who speaks of the Internet's "universality" (p. 449); in other words, potential offenders have unlimited capacity to get their messages into cyberspace, and such messages can be responded to and pursued any time both parties happen to be online.

It is also important to note that greetings in CMC generally allow for less use of visual and emotional cues than they would in face-to-face or even telephone interaction (Berson, 2003; Gottschalk, 2011; Walther, 1996). As a result, the recipient of a conversational opener during $\mathrm{CMC}$ has little more beyond the words themselves with which to judge the character and intentions of a conversational partner. Thus, one of the only ways the opening sequences of an online chat might alert an individual to potential danger is in the few cases that conversational openings are skipped entirely, in which case the first utterance might hint strongly at the person's intention(s). This powerful sense of anonymity is well documented on the Internet (Berson, 2003; Davidson \& 
Gottschalk, 2011; Gottschalk, 2011; Whittle et al, 2013), and its consequences will resonate in much of the following discussion.

\section{General Information Exchange and Topic Negotiation}

The data for the present study included several themes that served to either spark the conversations via discursively negotiating initial (non-sexual) topics or solicit/disclose basic (non-sexual) details about the conversational parties. These themes included 'initial solicitation of age, sex and/or location,' 'soliciting topic for discussion' and 'disclosing personal, non-sexual details.' These are modalities of interaction that one might expect any conversation, benign or predatory, to exhibit. While observance of these themes, in and of themselves, may not constitute evidence for cybergrooming, they become interesting in the broad scope of this research not only in how they ultimately progress to grooming strategies, but also as salient contributions to analysis of CMC in general. Many of these categories are common enough within the data that their conspicuous absence, rather than their presence, may signal a diversion from innocent conversational norms.

Initial solicitation of age, sex and/or location. The way an individual identifies his/herself is an important factor in relationship formation (Gibbs, Ellison, \& Heino, 2006; Zhou, 2012). In an online setting, identities are constructed by self-disclosure of personal and/or demographic information, and thus an expected facet of mutuallyanonymous CMC is the exchange of identifying information (Berson, 2003; Davidson \& Gottschalk, 2011; Gibbs et al., 2006; Gottschalk, 2011; Walther, 1996; Whitty \& Gavin, 2001). The data for this study support the case for normalization of such demographic exchanges. The most frequent 'first step' taken by the men in these chats toward gleaning 
such identifying information was soliciting basic age, sex and/or location information.

The fact that this theme occurred in $73 \%$ of sampled chats points to the near ubiquity of seeking this information, and the early emergence (positional mean $=8.59$ ) of such requests further cements it as a staple of mutually-anonymous CMC. Furthermore, this information was most often sought all at once, within one turn of talk, by use of the acronym ASL.

Subrahmanyam et al. (2004) identify the "a/s/l code" (p. 660) as a vehicle for how individuals express their identities online. ASL stands for 'age, sex and location' and is a discursive norm in mutually-anonymous online chat, used very commonly as an early way for strangers to begin to learn about each other (Smahel \& Subrahmanyam, 2007; Subrahmanyam et al., 2004; Whitty \& Gavin, 2001). While some of the men requested the ASL components separately, the majority who did solicit such information did so in one turn via the aforementioned acronym. Thirty-seven percent of the adults in the sample specifically requested ASL, and they usually did so early (positional mean = 5.76). This action was accomplished in numerous ways, including direct requests as well as providing one's own ASL information with the expectation that the action would be reciprocated. It should be noted that in many of the cases that did not feature an ASL request, the information was already provided by the decoy at the beginning of chat, either following or in place of a conversational opening. The fact that ASL is often provided without an actual request, or as a response to the other's ASL disclosure, speaks to this practice being an understood norm of anonymous CMC.

When the ASL acronym was not utilized, the men tended to ask for age and location information separately (Interestingly, only two out of 100 men inquired about 
the other party's gender independent of other demographics). Initial age requests were more common ( $21 \%$ of chats) than initial location inquiries (17\%). However, initial location inquiries occurred, on average, more than three lines earlier in chat. This could speak to the importance of determining victims' locations to the overall process of cybergrooming (see 'follow-up topicalization of 'child's' location' subsection). The fact that the range of any type of ASL request spanned only lines 1-29 has two distinct theoretical implications. The earliest occurrences, especially those at line 1 , lend validity to the idea posited by Subrahmanyam et al. (2004) that the normalization of this type of information seeking is such that it is considered acceptable to substitute an ASL inquiry for a traditional greeting. Furthermore, observing that the vast majority of these come early in chat, and never after line 29 , aligns with prior findings that basic personal information is a key for strangers to begin to find common ground for conversation (Berson, 2003; Gibbs et al., 2006; Smahel \& Subrahmanyam, 2007; Subrahmanyam et al., 2004; Walther, 1996; Whitty \& Gavin, 2001).

ASL in cybergrooming. The cybergrooming literature is surprisingly thin in terms of analyzing behaviors such as ASL, considering its relative ubiquity to online chats. The present research forwards the theory derived from findings of case studies like Marcum (2007), who noted ASL as one common early feature of cybergrooming chat, as well as findings identifying the exchange of personal information as a feature of cybergrooming (Berson, 2003; McGhee et al., 2011; Whittle et al., 2013). This theme and its features also fit into O'Connell's (2004) staged typology of online grooming. According to O'Connell, the "friendship forming stage" (p. 6) is the first of four successive but interchangeable stages of cybergrooming, and features the adult's attempts to learn more 
about the potential victim(s) and disclose enough personal information to make the child believe a trusting bond is beginning to form. Even if an abuser is not well versed in CMC norms, he may very quickly adopt some fairly obvious practices (such as ASL inquiries) so as to build rapport with potential victims by synchronizing his communicative behaviors with those of the child (Whittle et al., 2013). While ASL exchanges certainly must be included in any comprehensive typology of cybergrooming behavior, it may be nearly impossible to mark a standalone ASL utterance, in and of itself, as part of a grooming attempt.

Soliciting (non-sexual) topic for discussion. According to Tracy \& Robles (2009), “The nature of actuality - of things that have happened, or what things 'are' appears to be central to the meanings of "question"” (p. 132). Rollman and Parente (2001) observed that questioning statements facilitated more reciprocal communication in online chats than did any other type of utterance. Although the present study did not examine the decoys' discourse, the prevalence of questioning (of all types) suggests that the men are aware of this fact and use interrogatives as a way to spark, as well as sustain, conversation. Additionally, communication theory is rife with evidence that questioning demands the preferred response of a relevant answer (Curl \& Drew, 2008; Goodwin \& Heritage, 1990; Pomerantz, 1984; Sidnell, 2010; Steensig \& Drew, 2008). Thus, it is no surprise that generic questions regarding the current state of affairs according to each party in chat commonly appear relatively early in these chats. The theme of 'soliciting topic for discussion,' or simple questions like "what's up?", "what's going on?" and "how's life?" occurred in $36 \%$ of chats within the sample, and appeared, on average, just a few lines after ASL inquiries (positional mean $=11.56$ ). 
In order to analyze the role of interrogatives in how cybergroomers construct their behavior, one must look back to Goody (1978b), who posited that questions have both locutionary (information-seeking) and performative (social action) functions. While this first type of question is exceedingly generic and may be expected to be part of both online and offline discourse, as well as conversation without the end goal of grooming or luring, the intended or achieved social action must nonetheless be considered. On the surface, the locutionary function of asking 'what's up?' seeks an evaluation of the other party's current situation. However, the performative function of the same inquiry may go deeper. At the very least, asking generic questions early in chat improves the chances of reciprocal discourse and continuation of conversation (Campbell, 2009; Goodwin \& Heritage, 1990; Goody, 1978b; Sidnell, 2010; Steensig \& Drew, 2008; Tracy \& Robles, 2009).

Furthermore, as Olson et al (2007) point out, child sexual abuse features a onesided power differential in which the adult seeks to maintain authority and thereby manipulate the conversation toward his goals. Therefore, by establishing himself as the primary "questioner" in conversation, an abuser inserts himself into a role of discursive power. Since adjacency pairs, in their traditional form, are rendered less salient in synchronous CMC, Edelsky's (1981) notion of the conversational floor may be a better way to look at this phenomenon. Simpson (2005) conceptualizes the conversational floor as a three-pronged force including the topic, or "aboutness" of discourse, the social action of discourse, and the participants" "sense of what is happening" (p. 345). Not only do questions serve to set the topic in these chats, but they also have the potential to give the 'child' cues as to how they should behave during the chat session. Here, the abuser can 
set a precedent for himself as the conversational leader, thereby setting up the 'child' in the submissive role of responding to inquiries (which often become much more specified and personal later) in a preferred manner. Topic-seeking questions, then, tap into Simpson's (2005) idea that whoever controls the conversational floor in CMC has a sort of power over the overall direction of discourse.

However, true control and perceived control are very different in terms of discursive function. Recalling prior theory: "Successful grooming leaves the victim unaware that any process is underway" (McGhee et al., 2011, p. 105). Bennell, Alison, Stein, Alison, \& Canter (2001) noted that many child sexual abusers communicate in ways that preserve their true power over discourse but allow the victim to believe he/she has control. In the context of the present study, giving the 'child' a sort of token control over the first topic of conversation may serve just this function. Initial topic solicitation, then, seems to be a common first step in the grooming process. In terms of O'Connell's (2004) stages of cybergrooming, generic questions would best fit under the friendshipforming stage, though this could overlap with the relationship-forming stage, which is an extension of the former and features more specific questions regarding the child's life. Topic-soliciting questions might be more closely related to the notion of rapport building, during which an abuser mimics the child's discursive patterns so as to generate perceived commonality (Berson, 2003; Whittle et al., 2013). Rapport building can, in turn, facilitate grooming by lending a deceptive feeling of empathy (and therefore building trust) between abuser and potential victim (Bennell et al., 2001; Berson, 2003; Campbell, 2009; Craven et al., 2006; O’Connell, 2004; Olson, 2007; Whittle et al., 2013). 
Disclosing personal (non-sexual) details. The final component of general information exchange/topic negotiation involved the adults' unsolicited self-disclosures of a non-sexual nature. This theme was observed in $25 \%$ of chats and tended to emerge later than the other aforementioned themes (positional mean $=19.30$ ), though its relatively high standard deviation (10.03) reveals that there was wide variance in this theme's sequential positioning. Included here are all utterances in which the adult disclosed mundane details about their lives without the decoy having specifically asked. Conversationally, it makes sense that self-disclosures appeared later than conversational openers and other initial actions, and there is little evidence that this practice, in and of itself, heralds a grooming attempt or other malicious intent. However, taken as one part of the entire context, self-disclosure in this fashion lends itself to grooming practices.

One central tenet of luring communication theory is deceptive trust development (Olson et al., 2007). This is the process of building what appears to be a trustworthy persona so that the victim will feel more at ease with uncouth utterances and/or sexual advances. Self-disclosure is a crucial part of potential romantic relationships (Gibbs et al., 2006), and is generally considered an affiliative behavior, or one that promotes intersubjectivity (Sidnell, 2010) and mutual understanding (Goodwin \& Heritage, 1990). Since a CA approach demands consideration of how each utterance's perceived meaning affects speakers' relationships (Robinson, 2013), and talk reflects unconscious adoption of conversational norms (Holt, 2003), the present research must consider the potential effects of self-disclosure. The limited capacity for CMC to reveal cues commonly used to judge a person's sincerity and the veracity of their statements makes any such disclosure fall short of true self-disclosure in a traditional conversational sense (Walther, 1996). 
Instead, cybergroomers practice 'selective self-disclosure' (Gibbs et al., 2006) so as to present only the best-possible facets of personality. Gibbs et al. (2006) note that online daters who wish to pursue a relationship beyond their present interaction tend to perform affiliative behaviors like self-disclosure more than those who did not. Since all of the adults in this study's sample necessarily ended up pursuing further relations with their potential victims, it is safe to assume this is the case here. Campbell (2009) found that child sex abusers admitted pretending to be more social in order to increase positive reception of their advances, and the present results mirror cybergrooming literature in suggesting that groomers use such strategies to build rapport and trust via mutual selfdisclosure (Bennell et al., 2001; Campbell, 2009; O’Connell, 2004; Olson et al., 2007; Whittle et al., 2013).

\section{Digging Deeper: More Specified Inquiries.}

While the actions analyzed above, if taken out of context, may seem innocent and ordinary enough to be a part of any number of benign CMC exchanges, the themes analyzed henceforth begin to illustrate many of the more concrete features of cybergrooming. The themes included here ('specific questions regarding 'child's' life,' 'follow-up topicalization of 'child's' location' and 'seeking 'child's' age preference for sex/romance') are all interrogative in nature and support prior research suggesting that question-asking plays a key role in the cybergrooming process (Craven et al., 2006; Marcum, 2007; McGhee et al., 2011; O’Connell, 2004; Olson, 2007; Shannon, 2008; Whittle et al., 2013). These themes tended to (but did not always) emerge later in chat than those in the previous subsection. The 'obvious' cybergroomers forego greetings and topic negotiation and instead jump straight into these types of statements. 
Asking specific (non-sexual) questions. The theme 'specific questions

regarding 'child's' life' was the most commonly observed category within the sample, occurring 110 times across 55 transcripts. These inquiries did not include any sexual questions (see below), but solicited a wide range of information about the 'child's' life, such as family dynamics, school schedules, beliefs, non-sexual desires, preferred activities, etc. Their prevalence alone suggests that this theme may be central to cybergrooming behavior. Interestingly, this theme also had the highest density (2); once a man asked a specific question, he tended to follow this up with further questioning.

Questions demand answers. A plethora of research has revealed a strong compulsion to provide a conditionally-relevant response to a direct question, and that doing so promotes intersubjectivity and social cohesion (Goodwin \& Heritage, 1990; Goody, 1978; Schegloff, 1968; Schegloff, 1984; Sidnell, 2010; Steensig \& Drew, 2008; Tracy \& Robles, 2009). While the present study did not analyze decoys' responses to questions, this expectation is observably salient for the adults based on the sheer number of specific inquiries as well as the tendency to respond to unsatisfactory answers with follow-up questions.

As mentioned above, questioning on the part of the abuser is a very common aspect of grooming behavior. McGhee et al. (2011) specified 'exchange of personal information' as an expected facet of cybergrooming and noted that this category often involved topics like the child's friends and likes/dislikes: "The predators tend to use this information to indicate that they have something in common with the potential victim or to gauge their support structures" (p. 107). Similarly, Whittle et al. (2013) posits 'mutuality' as part of rapport building that "involves the offender learning about the 
young person's interests, beliefs and circumstance and the acceptance of these enabling a connection to be made" (p. 65). Marcum (2007) found that child sex offenders routinely ask very personal questions and tend to direct the conversation back to that subject matter if preferred responses are not provided. The present data fully support these claims. In fact, asking personal/specific questions almost seemed to act as something of a 'turning point' in some of these chats; perhaps once the adults verified the 'child's' willingness to answer personal inquiries they felt comfortable enough to move the conversational topic toward more relational or sexual themes. This also signals an early sign of successful deceptive trust development, in which the abuser "communicatively sets the stage for future sexual contact” (Olson et al., 2007, p. 241). Finally, specific questions fit nicely into O'Connell's (2004) relationship-forming stage of cybergrooming. Here, following (and possibly overlapping with) the friendship-forming stage, groomers "endeavor to create an illusion of being the child's best friend" (p. 6). Again, these questions seem to go beyond information seeking and more often serve the performative (Goody, 1978b) function of building up the trust enough to make it likely a child will reveal even more private details.

Follow-up location inquiries. This theme encapsulated one of the most apparent features of cybergrooming revealed by the present study. Almost half (44\%) of the men requested more specific information regarding the 'child's' physical location after initial ASL or location inquiries had been answered. These questions appeared, on average, about 17 lines into chat (well after initial location requests), and included inquiries about specific region (i.e. "where in cali?"), city or town (i.e. "what city") and even requests for very specified community or street-level details. The fact that this theme was quite dense 
(occurring 1.66 times per chat) further justifies its placement as a salient facet of cybergrooming. Furthermore, the evidence presented here suggests many of the men understand that a certain level of trust must be reached before the decoys will reveal such information; these were almost never the first interrogative observed and the subject was often re-introduced multiple times.

This theme does not overtly fit into any of O'Connell's (2004) stages of cybergrooming, though it might closely mirror some aspects of the friendship-forming stage. Here is where this model, for the first time, seems incomplete based upon the data for this study. In fact, none of the cybergrooming literature taps into this phenomenon with any level of specificity. Bennell et al. (2001) note the dichotomy of "autonomy versus control" (p. 158), in which abusers feign a certain level of handing control of the conversation over to the child, thereby discursively making the victim share some responsibility for the crime. Perhaps this strategy comes into play here, as the men might interpret willingness to divulge location as some type of consent or complicity. However, this implies that location follow-ups are almost purely performative in nature, when in fact these appear to be some of the only questions asked that may predominantly perform a locutionary function (Goody, 1978b). The very aggressive and repeated nature of some of the data within this theme suggest the men really do want this information, rather than asking for some other manipulative purpose.

Some prior research has suggested that groomers are very adept at collecting information about potential victims, and while the present data cannot speak to success in such endeavors, it certainly indicates the men very often believe this to be the case and consistently seek out such details (Berson, 2003). Certainly, deceptive trust development 
is central to ascertaining this type of specific information, though it seems intuitive that this line of inquiry would come after a certain level of trust had been built (Olson et al., 2007). The explication of groomers' intent by Briggs et al. (2011) may offer some insight at least into which types of abusers would aggressively pursue information about victims' locale. These authors split child sex offenders into "contact-driven" and "fantasy-driven" categories (p. 85). Location pursuits would seem to fit nicely into the contact-driven scheme, which is characterized by offenders whose communicative purpose is to set up an in-person meeting with the child. The problem in making this connection lies in the nature of the present study's data. Publically available Perverted Justice chatlogs all necessarily culminate in an attempted offline meeting; therefore it is impossible to derive such motivations from this data set. However, it is interesting to note that location pursuits occurred, on average, more than once per transcript; given that said transcripts included only the first 20 utterances by each adult, this finding is troubling and cannot be overlooked. Further research could shed more light on this theme's place in the cybergrooming behavioral phenomenon.

'Do you like older men?': Age-preference inquiries. Over a third (35\%) of the men within the sample made some sort of inquiry as to the 'child's' age preference(s) for romantic and/or sexual relationships. Such a pattern, in and of itself, lends validity to the notion that these data are indeed reflective of observable cybergrooming behavior, and its relatively early emergence (positional range $=5-40$ ) suggests that many of these men are wasting little time before making their intent and desires known. This theme was observed, on average, just past line 20 , or approximately halfway through each chat's sample quota. Thus these interrogatives offer one of the earliest discursive 'red flags' that 
may alert a potential victim of danger. Age-preference inquiries were fairly uniform and recognizable as such, but some variance was observed. For example, some asked directly about the decoys' age preference for relationships or sex or if they liked older men, while others framed such statements as self-deprecations (as in, 'am I too old?').

Regardless of syntax, the presence of age-preference inquiries during chat conversation strongly suggests at least two things. First, the individual posing the question is likely interested in a romantic and/or sexual relationship; there are few other reasons that this information, especially in the context of an adult-child stranger interaction, should be sought. Second, such statements make it clear that the asker knows that an age gap exists and that it might be a problem. While this does not amount to an admission of wrongdoing on the part of the adult, and it would be invalid to attempt to glean the perpetrators' moral stance on the issue, such an unusual inquiry intuitively suggests that the asker realizes some people (i.e. the child) might construe the age gap as morally reprehensible or at least sexually undesirable. The cybergrooming literature has done very little to identify this pattern as an associated behavior ${ }_{2}$ and next to nothing in terms of analysis. This gap must be filled before any theory can be considered comprehensive; this theme's emergence as a recurring feature of the present study's sample makes it valid and necessary to include it as a facet of cybergrooming.

While age-preference inquiries are not explicitly mentioned in the cybergrooming literature corpus, the theme (in some cases) may at least tenuously relate to some known facets of the behavior. For example, because an answer indicating that the child sees the age gap as undesirable would be tantamount to calling the man himself undesirable, an inquiry of this nature could be construed to exemplify the well-established strategy of 
discursively manipulating the child's moral views on adult-child relationships

(Buchanan, 2012; Craven et al., 2006; Lang \& Frenzel, 1988; Olson et al., 2007). This theme could also be tied to O'Connell's (2004) risk-assessment stage, but the present data are not conclusive enough to justify framing these statements as gauges of the child's likelihood to report abuse. In similar fashion, age-preference inquiries could, in some cases, be loosely related to a discursive variant of the 'accidental touch' strategy, by which abusers slowly introduce romantic/sexual behaviors (or broach the topic in CMC) in order to gauge the victim's willingness to participate (Craven et al., 2006; Elliott et al., 1995; Lang \& Frenzel, 1988). Again, these connections are far from concrete, and more research is needed to flesh this theme out in more detail.

Discursively, these questions could fulfill one or both of Goody's (1978) functions. In a locutionary sense, age-preference inquiries do seek exactly the information requested. In a performative role, such questions could serve as a non-overt way to express the adult's interest in pursuing sex. In a related view, they could also represent a method of establishing romantic/sexual interests as the conversational floor (Edelsky, 1981; Simpson, 2005). In terms of conversational norms, because these discursive units are inherently interrogative in nature they obligate the other party to provide a conditionally-relevant response. Furthermore, when asked in polar (yes/no) form, such questions make relevant a response that preserves face for the asker by indicating his age is acceptable (or at least not a problem); this is especially the case when the inquiry is self-deprecating (Goodwin \& Heritage, 1990; Schegloff, 1984; Sidnell, 2010; Tracy \& Robles, 2009). This makes age-preference inquiries a powerful 
manipulation tool for the cybergroomer, as well as a telling signal to informed potential victims that their conversational partner may be trying to groom them for sex.

\section{Overt Grooming and Sexual Advances}

The preceding subsection explicated themes and discourse that could, under most normal circumstances, be identified as cybergrooming behaviors. The four themes explored below ('seek visual,' 'compliment 'child's' appearance,' 'arrange further contact' and 'explicitly sexual statements') encapsulate almost undeniably predatory social actions, especially in the context of adult-child conversations. These tended to (but did not always) occur later in chat than those outlined above. Keeping in mind that the present study sampled only the first 20 lines of each adult's chat discourse, the fact that these emerge with any sort of regularity in the opening sequences of often very long chats not only make it difficult to explain most of them as anything but cybergrooming but $_{2}$ also lend validity to literature suggesting the Internet facilitates faster and more extreme self-disclosures and sexual or otherwise objectionable communication (Berson, 2003; Davidson \& Gottschalk, 2011; Davis, 2012; Gottschalk, 2011; Jewkes, 2010; Whittle et al., 2013).

'Pics' and other visual requests. The theme 'seek visual image of 'child"' emerged from the data with $40 \%$ prevalence but occurred 72 total times, meaning men who desired a visual often made multiple requests and making this one of the denser themes considered here. Its positional mean (22.49) places it very close to the average timing of other themes, such as 'seek age preference' and 'follow-up topicalization of 'child's' location,' that represent a potential 'turning point' from innocuous chat to cybergrooming. This theme is mostly comprised of direct solicitation of pictures (often 
called 'pics') of the 'child' and/or requests for the 'child' to appear on webcam video (often called 'cam'). It also includes instances of requests for the 'child's' physical description, but this was very rare. It should be noted that the Perverted Justice decoys often fulfilled such requests, and some appear to have posted a photo to their chat profile. However, this does not minimize the moral and legal issues that arise from an adult asking a child to transmit images of themselves online, especially considering that sexual topics had often been broached by this time and some men even explicitly asked for 'naughty' pictures. Such requests, when fulfilled, were overwhelmingly met with compliments on the 'child's appearance, providing even more evidence to mark such requests as aspects of grooming (see next subsection).

Requests for photographs and other media mark a departure from traditional notions of offline grooming; the multimodal capabilities of the Internet now allow for instant and unlimited exchange of pictures and video (Gottschalk, 2011; Quinn \& Forsyth, 2004; Wolak et al., 2008). Shannon (2008) found that up to $23 \%$ of sex offenders who targeted children persuaded victims to transmit sexual pictures and up to $37 \%$ requested webcam chat. The present study's data support this finding (Note that, for still images, Shannon's study counted only successful attempts to solicit a picture). Additionally Marcum's (2007) case study made multiple mentions of photo requests (as well as offers) by the adults, but her analysis largely failed to discuss this theme in any analytical detail. Finally, Wolak et al. (2008) note that some victims of online grooming reported being asked for explicit photos, but the number was incredibly low (4\%). All of the aforementioned studies posit these actions as a step toward the communicative desensitization (Olson et al., 2007) of the children to sexual advances and behaviors. The 
data presented here support these findings but to a greater degree, both in prevalence as well as in relation to cybergrooming as a construct.

In fact, the present findings indicate a departure from this framework in that most prior studies noted much higher incidence of the adult offering and/or sending explicit photos of themselves; this study found a much lower rate of this phenomenon, at least toward the beginning of chat. Finally, a large body of prior grooming literature noted groomers' propensity for sharing pornography (often featuring minors) with potential victims in order to desensitize them (Elliot et al., 2005; Lang \& Frenzel, 1988; Malesky, 2007; Olson et al., 2007; Whittle et al., 2013; Wolak et al., 2008). Analysis did not reveal this expected behavior whatsoever (again keeping in mind the data analyzed here represent only the very beginnings of chat).

Complimenting 'child's' appearance. Once the men in the sample received a photo of the 'child' or viewed one on an external profile site, they overwhelmingly tended to compliment the 'child's' appearance. Thirty-nine percent of the men did this, and like the requests for photographs that very often preceded the compliment, this theme was also very dense, totaling 69 occurrences $($ density $=1.77)$. Compliments emerged, on average, just before the halfway point of the sampled partition of the transcripts.

However, this statistic may have been affected by the handful of cases in which the adult offers a compliment within the first few lines of discourse, most likely because they had viewed the decoy's profile page before initiating chat. The way the adults worded these compliments also stood out. The vast majority of them were almost sexual in nature; the most common adjectives used, by far, within the units of this theme were along the lines of 'hot' and 'sexy.' Furthermore, several of the compliments mentioned the decoy's 
supposed age (i.e. "ur hot for a $13 \mathrm{yr}$ old"). Finally, some of the men went so far as to acknowledge that they may be doing something morally reprehensible for thinking the 'child' is attractive (i.e. "I feel dirty") in a neighboring turn.

Interestingly, compliments are not explicitly mentioned or analyzed in most of the cybergrooming literature. This is especially surprising given that several studies explore the transmission of visual images during the grooming process (Marcum, 2007; Shannon, 2008; Wolak et al., 2008). It is unclear whether these authors did not observe the same pattern following photo exchanges that the present study's data revealed or simply chose to lump compliments in with other trust-building aspects of grooming. Similarly, McGhee et al. (2011) make note of compliments as a potential category for analysis, but it is absent from their final model. Campbell (2012) actually included compliments specifically, along with a range of similar affectionate and trust-building utterances, in her "ingratiating" (p. 453) category of self-presentation strategies. Again, almost every cybergrooming study hints at the power of compliments by describing parts of the grooming process that involve special attention and affection, but none examine compliments, in and of themselves, with any specificity. However, their prevalence and density here justify an explication of compliments as their own thematic category.

Olson et al. (2007) define grooming as, "the subtle communication strategies that child sexual abusers use to prepare their potential victims to accept the sexual contact" ( $p$. 241). Without a doubt, complimenting the child's appearance easily fits into this broad definition. Compliments may also fit into behavioral strategies mentioned by several other authors, such as making the child feel good (Craven et al., 2006), building a perception of mutuality (Whittle et al., 2013), affection and reassurance (Bennell et al., 
2001), and O'Connell's (2007) "relationship-forming stage" (p. 6). The way

compliments were often framed with sexualized language also suggests some of these men used this strategy to normalize flirting or sexual discourse by making the child feel like an adult. Similar claims have been made by Bennell et al. (2001), who described the successful strategy of giving the victim perceived autonomy, and by Buchannan (2012), who found that some cybergroomers attempted to discursively convinced potential victims that they were mature enough to make adult decisions. By using words like 'sexy' and 'hot,' perpetrators are either consciously or unconsciously speaking in terms that a youthful victim would identify with adult behavior and perception. Thus, the way compliments are delivered, perhaps more than the fact that they are present, may be a useful research path.

Arranging further contact. The theme 'arranging further contact' emerged out of 2 categories: 'arrange meeting' and 'arrange other contact.' The former refers to attempts by the men to entice the 'children' to meet in person. Eighteen percent of the chats in the data set featured discourse initiated by the men directly requesting, or gauging the 'child's' interest in, a face-to-face meeting. The latter involves any attempt by the men to secure further or subsequent lines of communication with the 'children' (e.g. telephone, e-mail, social networks, etc.). While 'arrange other contact' emerged in just $12 \%$ of the chats, its relatively high density (1.58) indicates multiple attempts to achieve this goal tended to be made. Combined as a theme, this action emerged relatively often (28\% prevalence), considering this data contains only the very early portions of chat. Also notable is this theme's overall density (1.78), suggesting that the men interested in negotiating further contact early on were inclined to prioritize this goal. It is 
somewhat strange that this feature of cybergrooming is not mentioned more than it is in the literature; in fact it often seems to be treated more like an assumed goal of grooming, rather than an individual feature of the framework. I would argue that its emergence as early as line 8 of chat, coupled with its penchant for re-emergence and negotiation among and between many other themes, justifies deeper analysis.

The fact that 'arranging further contact' emerged so clearly was not surprising, in and of itself, in the context of the present data. Despite the grounded theory approach of this research, this social action, perhaps more than any other, had to be intuitively anticipated and carefully considered during analysis. Potentially clouding the validity of this theme is the issue that the Perverted Justice decoys' end goal is to specifically induce the solicitation of an in-person meeting. As such, this was one area in which the decoys' chat surrounding units resembling this theme was particularly salient. Thus this theme's validity was bolstered when analysis revealed that each of its 50 total occurrences were social actions clearly initiated by the men.

Arranging further contact aligns in principal with the second construct surrounding deceptive trust development, isolation, which has both a physical and psychological component (Olson et al., 2007). Arranging a meeting falls neatly in line with physical isolation, while establishing enough trust to convince the 'child' to exchange phone numbers and other contact information could be a step in the direction of mental isolation. If such a relationship is indeed perceived, access to more direct communication with the potential victim could lead to, or be a sign of, Olson's conceptualization of psychological isolation: "Perpetrators aim to create or enlarge the mental space between the targeted victim and the victim's support network so that the 
perpetrator can then step into that space" (p. 243). This model, however, is

predominantly based on offline luring strategies that fail to consider how the Internet inherently isolates a teen chatting online from the direct influence of said support networks, thereby removing some communicative barriers. Toward adapting this construct to fit cybergrooming, an updated model might largely eliminate the idea of physical isolation via strategies like "offering to babysit" (p. 242), and instead consider how a direct line of communication or in-person meeting might be negotiated without parents or guardians ever knowing a conversation took place.

'Arrange further contact' aligns with the 'approach' feature of cybergrooming described and anticipated by McGhee et al. (2011), as well as facets of perpetrators' risk assessment process outlined by Whittle et al. (2013). Whittle et al. note that many perpetrators attempt to mitigate risk by using private e-mail and phone numbers, and some who try to meet victims in person prefer to do so away from home. While it is not clear whether arranging other contacts in the present data relates more to risk assessment or to psychological isolation, physical isolation via arranging a meeting becomes interesting if taken in the broader context and considered in relation to other themes within the present data. Looking back at the patterning of 'follow-up topicalization of 'child's' location' reveals striking similarity to that of the present theme. Men who tended to pursue the specific locations of the 'children' often did so repeatedly, as they did also when pursuing meetings and other contact. This lends support to the idea that some cybergroomers prioritize meeting their victims in person, while others seem more focused on discursively exploring their desires online, at least in the beginning of the chats analyzed here (Briggs et al, 2011; Marcum, 2007; Shannon, 2008). Specifically, 
those who repeatedly tropicalize potential victims' locations and/or attempt to set up a meeting right away likely fit into the contact-driven, as opposed to fantasy-driven, offender classification (Briggs et al., 2011). This conclusion also supports Shannon's (2008) finding that $96 \%$ of cybergrooming offenders who met victims offline attempted to establish phone communication with their victims and $36 \%$ expressed desire to meet offline online interaction, compared to $5 \%$ and $11 \%$, respectively, for those who had only online contact. The data here similarly indicate a relatively clear split between cybergroomers who pursue further contact immediately and those who do not. Thus, an adult attempting to solicit further contact with a child online, especially when coupled with seeking specific location information, must be considered one of many salient features of cybergrooming and can serve as a valuable behavioral indicator to potential victims.

Sexual communication in cybergrooming. Thirty-five percent of the chatlogs analyzed for this study progressed to sexual topics within the first 20 lines spoken by the adults. The theme 'explicitly sexual statements' encompassed three categories: 'sexual questions,' 'sexual self-disclosure' and 'sexual statement.' By far the most common of these was 'sexual question,' as $26 \%$ of the men asked the decoy some form of sexualized inquiry, ranging from experiences and sexual history to desires and preferences. While this theme was expected to emerge given the context of the data, it becomes truly poignant in the way its patterning sets itself apart from all other themes included here. Analysis revealed that the men's explicitly sexual comments made up, by far, the most wide-ranging and dense theme. Sexualized discourse was observed as early as line 2 and as late as line 50; thus the partial conversations in the sample ran the gamut from 
immediate sexualization to building up to the topic, and some men did not mention sex at all in their first 20 lines. In addition, this theme's incredibly high density (2.71) indicates that once a sexual topic was broached, the conversation was overwhelmingly likely to continue in that direction. Furthermore, this was the only theme that could clearly be marked as progressive and continuous in nature, with some of the men sending line after line of increasingly explicit discourse.

Sexual discourse is, obviously, a major theme throughout the grooming literature, and the present study is no exception. Beginning with traditional, pre-Internet grooming research and progressing through the most recent cybergrooming literature reveals that the role of sexual discourse becomes much more important in an online context. Earlier research focused more on the 'accidental touch' and subsequent gauging of the victim's reaction as a way to introduce sex; some also mentioned exposing the child to pornography and adult materials to normalize the topic (Lang \& Frenzel, 1988, Elliott et al., 1995). While a few of the men seemed to attempt a similar version of this strategy discursively by mentioning a sexual topic and awaiting the decoy's response (i.e. chat \#67: "I wont talk about sex because you a virgin and never saw a cock"), the fact that the vast majority who mentioned sex did so repeatedly and often in very explicit language strongly supports the notion of online communication is inherently disinhibited (Berson, 2003; Davidson \& Gottschalk, 2011; Gottschalk, 2011; Jewkes, 2010; Whittle et al., 2013). Therefore, cybergroomers can discursively normalize sex as a topic and establish it as the conversational floor, so that the idea is firmly cemented before any in-person meeting is set up. 
The most useful theoretical lens with which to examine cybergrooming's

sexual discourse features may be related to the concept of communicative

desensitization - the progressive process of normalizing sexual subject matter in the mind of the victim (Buchanan, 2012; Craven et al., 2007; McGhee et al., 2011; O'Connell, 2004; Olson et al, 2007; Whittle et al., 2011). The way some of the men progressed (albeit very quickly) from quasi-sexual statements and questions to very explicit descriptions of sex acts (see Example 35, Results section), supports this notion. Evidence also emerged to support the idea of reframing sexualized talk as either sex education or evidence of relational trust, which can not only serve to normalize sexual discourse but also establish a sort of teacher-student power dynamic between perpetrator and victim (Buchanan, 2012; Campbell, 2009; Craven et al., 2006; Olson et al., 2007). Much of this explication points back to the negotiation of power within cybergrooming behavior, and the fact that sexual questions made up this theme's most prevalent feature speak to this claim (68 of the 95 sexual utterances were interrogatives). Sexual questions in this study often cascaded from general inquiries (i.e. chat \#4: "would you like me to rub your back for you?") to extreme questions and statements (i.e. chat \#4, nine lines later: "how about your slit?") as the men sought more and more description of the 'children's' sexual experiences and desires while being more specific about their own. This aligns with Marcum's (2007) analysis of cybergrooming cases, in which one of the subjects "seemed to derive pleasure from dominating the conversation with his victim by questioning her about her sexual past" (p. 111). From a more conversational standpoint, questions can imply a certain type of relationship between parties (Robinson, 2013). It seems like this also holds true here, as sexual questions were a common way to broach sexual discourse, 
which rarely ceased once introduced. Thus, sexual questions in cybergrooming work to establish a sexualized conversational floor, usually with a severe imbalance of power, between adult and potential victim.

While the online-specific cybergrooming literature is rife with documentation of the sexuality of these types of interactions, it more often than not treats it as a stage to be approached, rather than a potential conversational floor. For example, O'Connell's (2004) fifth and final stage of cybergrooming is the "sexual stage" (p. 7). Like the present study, she notes that this stage is often introduced with questions that move from almost innocuous to obscene in nature. However, her claim that the adult, by this time, has typically established a deep perception of trust with the victim, cannot be supported by the data for this study. With over a third introducing sex by their $20^{\text {th }}$ statement, the claim that the sexual stage is typically reached only after a process of trust building is not generalizable. However, some patterning is evident in some of the cases. This comes closer to supporting Whittle et al. (2013), who suggest the sexual context of a cybergrooming interaction depends more upon the nature of the perpetrator. Those who brought up sex almost immediately would thus be categorized as "hyper-sexualized," and those that did not would fall on a continuum somewhere between "intimacy seeking" and “adaptable" (p. 65).

The present study can only partially support the aforementioned 'staged' construct; it is evident in some cases but clearly absent in others (whether because of immediate emergence or none at all). The prevalence found herein of sexual discourse came strangely close to the number reported by Briggs et al. (2010), who found that $35.3 \%$ (compared to $35 \%$ in the present study) of cybergroomers engaged in sexual 
discourse. However, since sex was mentioned by over a third of the men within their first 20 utterances, it seems likely that this number would have grown steadily had analysis delved deeper into conversations; this points to the validity of the three-pronged offender types outlined by Whittle et al. (2013), in which the adult's characteristics have more impact upon the progression of sexual discourse than does timing. Given the salience of the online disinhibition effect, the prevalence of explicit themes found here within just the early parts of chat, and the propensity for sexual topics to persist once broached, I would argue that sexualized statements are a landmark feature of cybergrooming. Since sexual communication is ubiquitous online (Davidson \& Gottschalk, 2011; Gottschalk, 2011), and sometimes even considered informative or even healthy (Jewkes, 2010), further research is necessary to delineate features that clearly mark predatory or otherwise malicious behavior. 


\section{Chapter 6: Conclusion}

The intensive research detailed in this study marks an important step toward understanding cybergrooming behavior. Analyzing and documenting how to recognize the early stages of predatory online behavior is a crucial public safety need, especially in an era in which young people can communicate online with strangers around the clock and largely without supervision. Thus, the present typography of initial cybergrooming actions represents an impactful tool for educators, youth, parents, law enforcement and the general public. The Internet, despite its many features that facilitate this type of behavior, does offer one significant advantage in protecting children from predation: communication can be cut off at any time by simply closing the chat window. While young people can still be damaged psychologically by the behaviors exhibited in this study's data set, this effect can be minimized by early recognition and termination of such conversations. Lowering risk for abuse via cybergrooming will often rely on children's knowledge of its indicators and recognition of their own agency in ceasing involvement in this type of discourse before revealing any personal information.

The 11 themes outlined and explicated herein strongly suggest that cybergrooming is not, as some prior research suggests, rather easily recognizable as a staged process moving from the non-sexual end of the spectrum to the sexual. Instead, I posit a framework of related discursive behaviors often observed in naturally-occurring adult-child $\mathrm{CMC}$ of a sexual nature. While many of the cases do exhibit something akin to stages - moving from conversational openings and greetings, through the gathering of more specified information, and ending in overt requests to meet or sexual advances - the fact is that most of these themes were found at almost any point in chat. Their positional 
means offer a rough picture of the sequencing of such discourse, but their high positional standard deviation suggests one must be cautious before adopting this framework as anything resembling a linear progression. Rather, I would call attention to certain themes that can act as relatively easy to identify 'red flags.' Such indicators would definitely include: any aggressive inquiry where an adult requests specific details about a child's location, topicalization of age preference for romance and/or sex, requests for pictures or video chat, appearance compliments (especially those framed in sexualized or 'adult' language), requests for in-person meetings and/or other contact information, and overtly sexual language of any fashion. All of the chats analyzed in the present study contain at least one of these themes. Adults who aggressively control the conversation and constantly steer the 'conversational floor' toward sexual topics are the easy ones to spot. However, one must keep in mind that while many groomers do roughly follow a progression from 'normal' chat dialogue to cybergrooming, some move much more slowly or quickly, and most overlap or revisit many of these themes throughout discourse.

Limitations and future research. Despite this study's breadth of sample and detailed analysis, it was necessarily limited by a number of factors. Due to time and resource restrictions, this research was only able to include cases in the sample set which were already established as examples of cybergrooming behavior (Perverted Justice only publishes transcripts of perpetrators convicted of related crimes). This was advantageous in that it provided a rich and conceptually-relevant data source, but disadvantageous in that analysis was not able to compare this behavior with what might be considered 'normal, everyday' synchronous CMC (or chat with no predatory or deceptive motives). 
In addition, though the sample's breadth facilitated heightened generalizability and a specific focus on the initiation of cybergrooming, this left out a plethora of subsequent turns at talk. These chats typically went on for hours, and some for days or weeks; thus many potentially valuable observations throughout later parts of chat were not made. The present study also did not directly consider the decoys' contributions to chat (though it was sometimes necessary to consider their contextual bearing on the adults' turns). The fact that they were creating false identities added too many layers of deception and motive to fall under the scope of this analysis, so this study is unable to speak to how an actual child or teen may navigate discourse in this situation. Finally, no data were available concerning the demographics, criminal history or any other information about the men, beyond what was self-disclosed in chat.

Detailed analyses like those performed in this study have the potential to contribute invaluable information to educate the public, and particularly young people who are active online, about what conversational patterns can serve as 'red flags' when communicating with strangers over the Internet. Future research could further this cause by offering a comparative analysis of the patterns, structure and social actions found in 'normal' synchronous CMC with those discovered in cybergrooming discourse. Another useful direction would be to utilize qualitative analysis software (as this study used Atlas.ti) to delve deeper into transcripts such as those in the present sample; synthesizing breadth and depth of analytical findings would provide a more complete framework for this behavior. Similarly, considering both parties' contributions to the chat would allow research to tap into the full spectrum of organization, preference and sequencing in these chats. Finally, interviews with convicted or admitted cybergroomers, and especially their 
victims (no study, to my knowledge, has done this), are necessary to add the emic perspective to the behavior associated with this phenomenon. 


\section{References}

Annells, M. (1996). Grounded theory method: Philosophical perspectives, paradigm of inquiry, and postmodernism. Qualitative Health Research, 6(3), 379-393.

Becker, H. S. (2001). The epistemology of qualitative research. In R. Emerson (Ed.), Contemporary field research (pp. 317-330). Prospect Heights, IL: Waveland Press.

Bennell, C., Alison, L., Stein, K., Alison, E., \& Canter, D. (2001). Sexual offenses against children as the abusive exploitation of conventional adult-child relationships. Journal of Social and Personal Relationships, 18(2), 155-171.

Berson, I. R. (2003). Grooming cybervictims. Journal of School Violence, 2(1), 5-18.

Blumer, H. (1969). Symbolic interactionism: Perspective and method. Englewood Cliffs, NJ: Prentice Hall.

Bowen, G. A. Grounded theory and sensitizing concepts. International Journal of Qualitative Methods, 5(3), 1-9.

Briggs, P., Simon, W., \& Simonsen, S. (2010). An exploratory study of Internet-initiated sexual offenses and the chat room sex offender: Has the Internet enabled a new typology of sex offender? Sexual Abuse: A Journal of Research and Treatment, 23(1), 72-91.

Buchanan, L. A. (2012, November). "Sorryifi am too old :(": Diologic struggle within pedophilic online solicitation communication. Paper presented at the National Communication Association conference, Orlando.

Button, G., \& Casey, N. (1985). Topic nomination and topic pursuit. Human Studies, 8, 3-55. 
Campbell, A. M. (2009). False faces and broken lives: An exploratory study of the interaction behaviors used by male sex offenders in relating to victims. Journal of Language and Social Psychology, 28(4), 428-440.

Craven, S., Brown, S., \& Gilchrist, E. (2006). Sexual grooming of children: Review of literature and theoretical considerations. Journal of Sexual Aggression, 12(3), 287-299.

Curl, T. S., \& Drew, P. (2008). Contingency and action: A comparison of two forms of requesting. Research on Language and Social Interaction, 41(2), 129-153.

Darmody, M., \& Byrne, D. (2006). An introduction to computerised analysis of qualitative data. Irish Educational Studies, 25(1), 121-133.

Davidson, J., \& Gottschalk, P. (2011). Characteristics of the Internet for criminal child sexual abuse by online groomers. Criminal Justice Studies, 24(1), 23-36.

Davis, K. (2012). Friendship 2.0: Adolescents' experiences of belonging and selfdisclosure online. Journal of Adolescence, 35, 1527-1536.

Deslauriers-Varin, N., \& Beauregard, E. (2010). Victims' routine activities and sex offenders' target selection scripts: A latent class analysis. Sexual Abuse: A Journal of Research and Treatment, 22 (3), 315-342.

Edelsky, C. (1981). Who's got the floor? Language in Society, 10(3), 383-421.

Elliott, M., Browne, K., \& Kilcoyne, J. (1995). Child sexual abuse previention: What offenders tell us. Child Abuse \& Neglect, 19(5) 579-594.

Emerson, R. M. (2001). Introduction: The face of contemporary ethnography. In R. Emerson (Ed.), Contemporary field research (pp. 27-53). Prospect Heights, IL: Waveland Press. 
Finkelhor, D. (1997). Child sexual abuse. Challenges facing child protection and mental health professionals (M. Margreiter \& K. Henschel, Trans.). In E. Ullmann \& W. Hilweg (Eds.), Childhood and Trauma: Separation, abuse, war (pp. 101115). Aldershot, England: Ashgate.

Gergen, K. J., (1985). The social constructionist movement in modern psychology. American Psychologist, 40(3), 266-275.

Gibbs, J. L., Ellison, N. B., \& Heino, R. D. (2006). Self-presentation in online personals: The role of anticipated future interaction, self-disclosure, and perceived success in Internet dating. Communication Research, 33, 152-177.

Glaser, B. G., \& Strauss, A. L. (1967). The discovery of grounded theory: Strategies for qualitative research. New York: Aldine De Gruyter.

Gonzalez-Lloret, M. (2011). Conversation analysis of computer-mediated communication. Calico Journal, 28(2), 308-325.

Goodwin, C., \& Heritage, J. (1990). Conversation analysis. Annual Review of Anthropology, 19, 283-307.

Goody, E. N. (1978a). Introduction. In E. N. Goody (ed.), Questions and politeness: Strategies in social interaction (pp. 1-16). Cambridge, U.K.: Cambridge University Press.

Goody, E. N. (1978b). Towards a theory of questions. In E. N. Goody (ed.), Questions and politeness: Strategies in social interaction (pp. 17-43). Cambridge, U.K.: Cambridge University Press. 
Gottschalk, P. (2011). A dark side of computing and information sciences:

Characteristics of online groomers. Journal of Emerging Trends in Computing and Information Sciences, 2(9), 447-455.

Hancock, J. T., \& Dunham, P. J. (2001). Language use in computer mediated communication: The role of coordination devices. Discourse Processes, 31(1), 91-110.

Hansen, C. (2007). To Catch a Predator. New York: Penguin Group.

Herring, S. (1999). Interactional coherence in CMC. Journal of Computer-Mediated Communication, 4(4). Retrieved from http://jcmc.indiana.edu/vol4/issue4/herring.html

Holt, R. (2003). Bakhtin's dimensions of language and the analysis of conversation. Communication Quarterly, 51(2), 225-245.

Holt, T. J., Blevins, K. R., \& Burkert, N. (2010). Considering the pedophile subculture online. Sex Abuse: A Journal of Research and Treatment, 22(1), 3-24.

Hwang, S. (2008). Utilizing qualitative data analysis software: A review of Atlas.ti. Social Science Computer Review, 26(4), 519-527.

Jewkes, Y. (2010). Much ado about nothing? Representations and realities of online soliciting of children. Journal of Sexual Aggression, 16(1), 5-18.

Lang, R. A., \& Frenzel, R. R. (1988). How sex offenders lure children. Sex Abuse: A Journal of Research and Treatment, 1, 303-317.

Longo, R. E., \& Groth, A. N. (1983). Juvenile sexual offenses in the histories of adult rapists and child molesters. International Journal of Offender Therapy and Comparative Criminology, 27, 150-155. 
Lu, C., \& Shulman, S. W. (2008). Rigor and flexibility in computer-based qualitative research: Introducing the Coding Analysis Toolkit. International Journal of Multiple Research Approaches, 2(1), 105-117.

Malesky Jr., L. A. (2007). Predatory online behavior: Modus operandi of convicted sex offenders in identifying potential victims and contacting minors over the Internet. Journal of Child Sexual Abuse, 16(2), 23-32.

Marcum, C. D. (2007). Interpreting the intentions of Internet predators: An examination of online predatory behavior. Journal of Child Sexual Abuse, 16(4), 99-114.

Markman, K. M. (2009). So what shall we talk about: Openings and closings in chatbased virtual meetings. Journal of Business Communication, 46(1), 150-170.

McGhee, I., Bayzick, J., Kontostathis, A., Edwards, L., McBride, A., \& Jakubowski, E. (2011). Learning to identify Internet sexual predation. International Journal of Electronic Commerce, 15(3), 103-122.

Mitchell, K. J., Wolak, J., \& Finkelhor, D. (2005). Police Posing as Juveniles Online to Catch Sex Offenders: Is It Working?. Sexual Abuse: A Journal of Research and Treatment, 17(3), 241-267.

Morgan, G., \& Smircich, L. (1980). The case for qualitative research. The Academy of Management Review, 5(4), 491-500.

O'Connell, R. (2004). A typology of child cybersexploitation and online grooming practices. University of Central Lancashire: Cyberspace Research Unit.

Olson, L.N., Daggs, J.L., Ellevold, B.L., \& Rogers, T.K.K. (2007). Entrapping the innocent: Toward a theory of child sexual predators' luring communication. Communication Theory, 17, 231-251. 
Pillet-Shore, D. (2011). Doing introductions: The work involved in meeting someone new. Communication Monographs, 78(1), 73-95.

Pomerantz, A. Agreeing and disagreeing with assessments: Some features of preferred/dispreferred turn shapes. In J. M. Atkinson \& J. Heritage (Eds.), Structures of social action: Studies in conversation analysis (pp. 57-101). Cambridge, U.K.: Cambridge University Press.

Quayle, E., \& Taylor, M. (2003). Model of problematic Internet use in people with a sexual interest in children. Cyberpsychology \& Behavior, 6(1), 93-106.

Quinn, J., \& Forsyth, C. (2005). Describing sexual behavior in the era of the internet: a typology for empirical research. Deviant Behavior, 26(3), 191-207.

Rintel, E., Mulholland, J., \& Pittam, J. (2001). First things first: Internet relay chat openings. Journal of Computer-Mediated Communication, 6(3), Retrieved from http://onlinelibrary.wiley.com/doi/10.1111/j.1083-6101.2001.tb00125.x/full

Robertiello, G., \& Terry, K. J. (2007). Can we profile sex offenders? A review of sex offender typologies. Aggression and Violent Behavior, 12, 508-518.

Robinson, J. (2013). Introducing conversation analysis. In J. F. Nussbaum (Ed.), Readings in communication research methods: From theory to practice (pp.95109). San Diego, CA: Cognella.

Rollman, J. B., \& Parente, F. (2001). Relation of statement length and type and type of chat room to reciprocal communication on the internet. CyberPsychology \& Behavior, 4(5), 617-622.

Sacks, H., Schegloff, E. A., \& Jefferson, G. (1974). A simplest systematics for the organization of turn-taking for conversation. Language, 50(4), 696-735. 
Schegloff, E. A. (1968). Sequencing in conversational openings. American Anthropologist, 70(6), 1075-1095.

Schegloff, E. A. (1984). On some questions and ambiguities in conversation. In J. M. Atkinson \& J. Heritage (Eds.), Structures of social action: Studies in conversation analysis (pp. 28-52). Cambridge, U.K.: Cambridge University Press.

Schegloff, E. A. (2007). Sequence organization in interaction: Volume 1: A primer in conversation analysis (Vol. 1). Cambridge University Press.

Segerdahl, P. (1998). Scientific studies of aspects of everyday life: The example of conversation analysis. Language \& Communication, 18, 275-323.

Shannon, D. (2008). Online sexual grooming in Sweden - Online and offline sex offences against children as described in Swedish police data. Journal of Scandinavian Studies in Criminology and Crime Prevention, 9, 160-180.

Sidnell, J. (2010). Conversation analysis: An introduction. West Sussex, U.K.: WileyBlackwell.

Silverman, D. (2006) Interpreting qualitative data: Methods for analyzing talk, text and interaction. Thousand Oaks, CA: Sage Publications.

Simpson, J. (2005). Conversational floors in synchronous text-based CMC discourse. Discourse Studies, 7(3), 337-361.

Smahel, D., \& Subrahmanyam, K. (2007). “Any girls want to chat press 911”: Partner selection in monitored and unmonitored teen chat rooms. CyberPsychology \& Behavior, 10(3), 346-353.

Steensig, J., \& Drew, P. (2008). Introduction: Questioning and affiliation/disaffiliation in interaction. Discourse Studies, 10(1), 5-15. 
Strauss, A., \& Corbin, J. (1998). Basics of qualitative research: Techniques and procedures for developing grounded theory. Thousand Oaks, CA: Sage Publications.

Subrahmanyam, K., \& Greenfield, P. (2008). Online Communication and Adolescent Relationships. The Future of Children, 18(1), 119-146.

Subrahmanyam, K., Smahel, D., \& Greenfield, P. (2006). Connecting developmental constructions to the Internet: Identity presentation and sexual exploration in online teen chat rooms. Developmental Psychology, 42(3), 395-406.

Tang, J. (2007). Approaching and leave-taking: Negotiating contact in computermediated communication. ACM Transactions on Computer-Human Interaction, 14(1), 1-26.

Tracy, K., \& Robles, J. (2009). Questions, questioning, and institutional practices: An introduction. Discourse Studies, 11(2), 131-152.

Wachs, S., Wolf, K., \& Pan, C. (2012). Cybergrooming: Risk factors, coping strategies and associations with cyberbullying. Psicothema, 24, 628-633.

Walsh, W. A., \& Wolak, J. (2005). Nonforcible Internet-related sex crimes with adolescent victims: Prosecution issues and outcomes. Child Maltreatment, 10(3), $260-271$.

Walther, J. B. (1996). Computer-Mediated Communication: Impersonal, interpersonal, and hyperpersonal interaction. Communication Research, 23(1), 3-43.

Webster, S., Davidson, J., Bifulco, A., Gottschalk, P., Caretti, V., Pham, T., \& GroveHills, J. (2010). European Online Grooming Project, Scoping Report. Brussels: European Commission Safer Internet Plus Programme. 
Whittle, H., Hamilton-Giachritsis, C., Beech, A., \& Collings, G. (2013). A review of online grooming: Characteristics and concerns. Aggression and Violent Behavior, $18(1), 62-70$.

Whitty, M., \& Gavin, J. (2001). Age/sex/location: Uncovering social cues in the development of online relationships. CyberPsychology \& Behavior, 4(5), 623630.

Wolak, J., Finkelhor, D., \& Mitchell, K. (2004). Internet-initiated sex crimes against minors: Implications for prevention based on findings from a national study. Journal of Adolescent Health, 35(5), 424.e11-424.e20.

Wolak, J. Finkelhor, D., Mitchell, K. J., \& Ybarra, M. L. (2008). Online 'predators' and their victims: Myths, realities, and implications for prevention and treatment. American Psychologist, 63(2), 111-128.

Zhou, Y. (2012). Opening a relationship online (published master's thesis). Linkoping University, Linkoping, Sweden. 


\section{Appendix A: Full Sample}

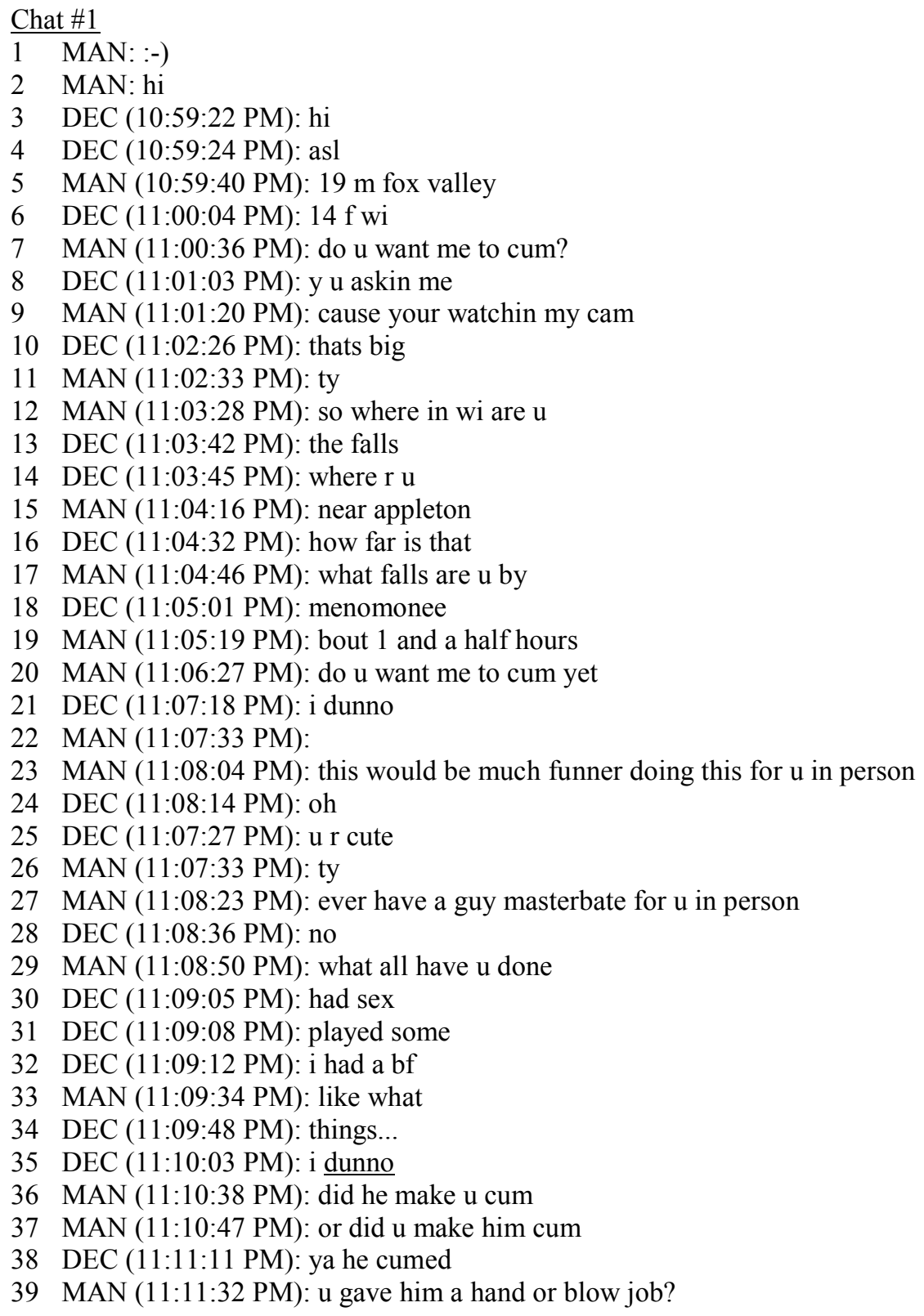

\section{$\underline{\text { Chat } \# 2}$}

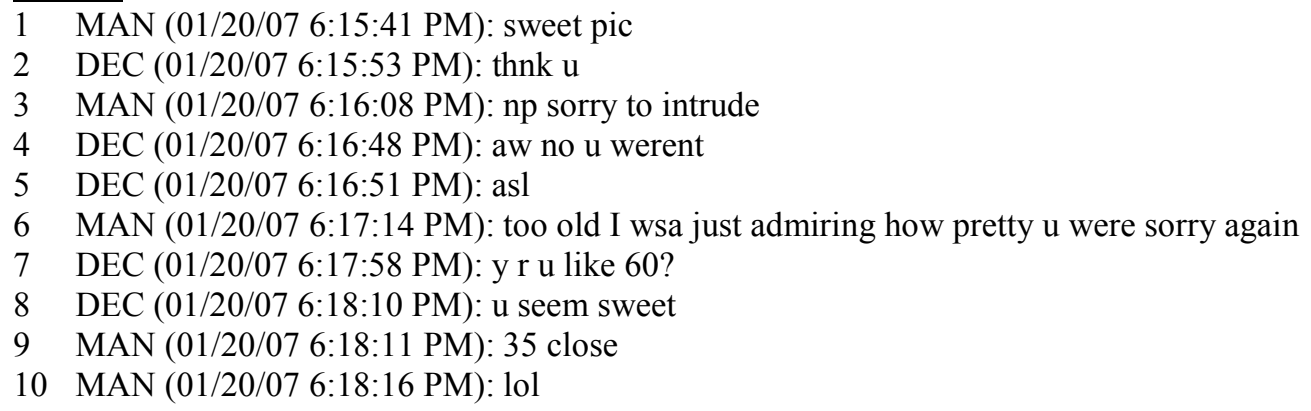


11 DEC (01/20/07 6:18:21 PM): thats not old lol

12 DEC (01/20/07 6:18:25 PM): ur funny

$13 \operatorname{MAN}(01 / 20 / 07$ 6:18:40 PM): hey I am a kid at heart

14 MAN (01/20/07 6:18:40 PM): lol

$15 \operatorname{DEC}(01 / 20 / 07$ 6:18:50 PM): lol u didnt seem like a grampa

16 MAN (01/20/07 6:19:20 PM): nope I dont even have any kids that I am aware of

17 MAN (01/20/07 6:19:22 PM): lol

18 DEC (01/20/07 6:19:31 PM): lol

$19 \operatorname{MAN}(01 / 20 / 07$ 6:20:04 PM): so how are $u$ this fine night

20 DEC (01/20/07 6:21:11 PM): im ok

21 DEC (01/20/07 6:21:14 PM): kinda bored

$22 \operatorname{MAN}(01 / 20 / 07$ 6:21:27 PM): bummer

23 DEC (01/20/07 6:21:37 PM): yeh how bout u

$24 \operatorname{MAN}(01 / 20 / 07$ 6:21:39 PM): so what do u do for fun when u can

25 MAN (01/20/07 6:21:58 PM): if I were any better it would be a sin thanks

26 MAN (01/20/07 6:23:38 PM): I scare u off already

27 DEC (01/20/07 6:24:01 PM): lol no sorry

28 DEC (01/20/07 6:24:15 PM): evryone keeps IMing me im tryin to get rid fo em

29 MAN (01/20/07 6:24:34 PM): thought $u$ fell a sleep on me:((

30 MAN (01/20/07 6:24:46 PM): oops sorry I can leave u alone if $u$ like

$31 \operatorname{DEC}(01 / 20 / 07$ 6:24:47 PM): lol

32 DEC (01/20/07 6:24:56 PM): no

33 MAN (01/20/07 6:25:43 PM): so may I be so bold to ask do u have a BF

$34 \operatorname{DEC}(01 / 20 / 07$ 6:26:25 PM): no

$35 \operatorname{DEC}(01 / 20 / 07$ 6:26:31 PM): i mean u can but i dont lol

36 MAN (01/20/07 6:26:55 PM): WOW if you dont mind me saying I am surprised as hell

37 MAN (01/20/07 6:27:17 PM): u are such a pretty woman and those eyes are fanaminal

38 DEC (01/20/07 6:27:33 PM): aw thnk u

39 DEC (01/20/07 6:27:54 PM): im 14/f tho u prolly dont wanna talk to me nemore

40 MAN (01/20/07 6:28:18 PM): well okay u are young if u dont mind I am okay

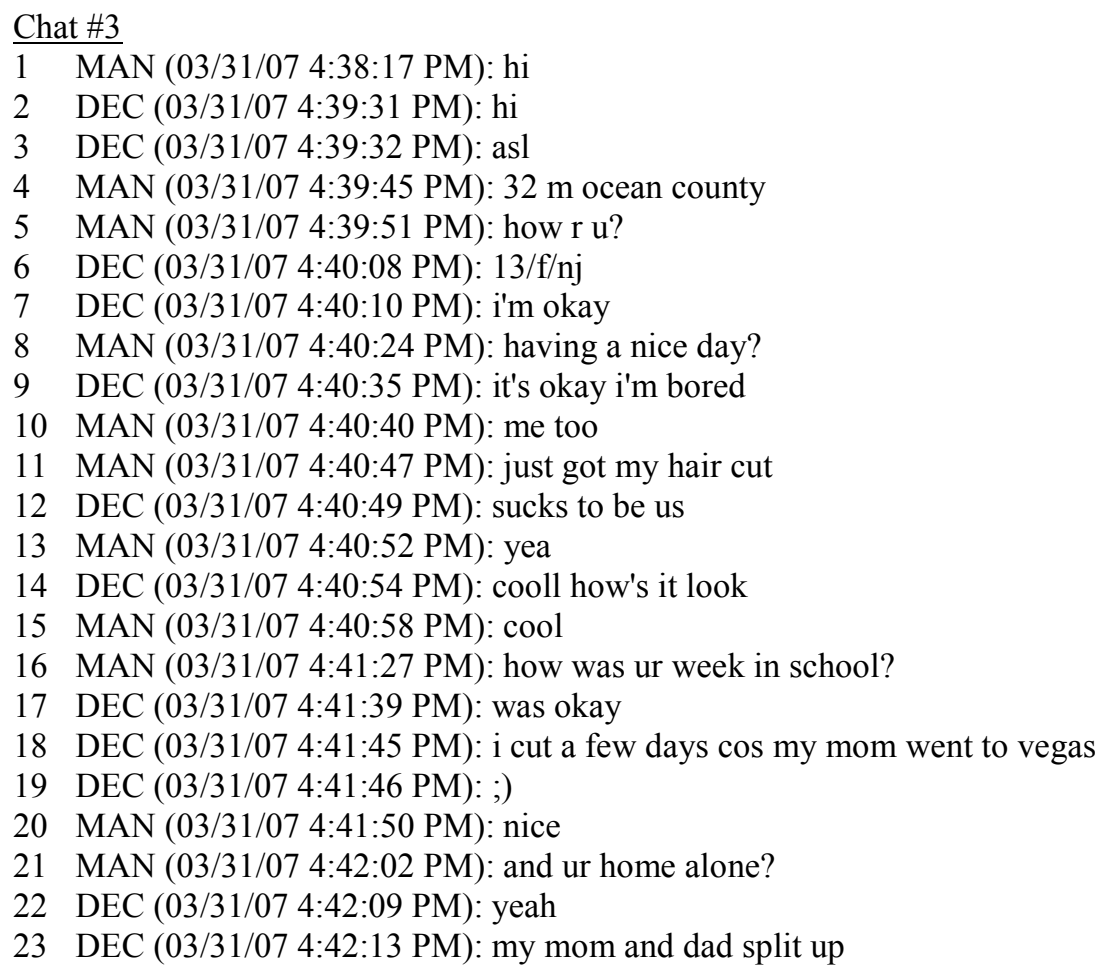




\section{CYBERGROOMING}

$24 \operatorname{DEC}(03 / 31 / 07$ 4:42:16 PM): that's why we movedhere

25 MAN (03/31/07 4:42:17 PM): sry

26 MAN (03/31/07 4:42:26 PM): where u live?

27 DEC (03/31/07 4:42:56 PM): 732

28 MAN (03/31/07 4:43:07 PM): ok

29 DEC (03/31/07 4:43:24 PM): got big plans?

30 MAN (03/31/07 4:43:31 PM): no

31 MAN (03/31/07 4:43:35 PM): u?

32 DEC (03/31/07 4:43:40 PM): me neither

33 MAN (03/31/07 4:43:52 PM): like older guys?

34 DEC (03/31/07 4:44:23 PM): i like guys who $r$ nice

35 DEC (03/31/07 4:44:25 PM): $r$ u nic?

36 DEC (03/31/07 4:44:27 PM): nice

37 DEC (03/31/07 4:44:29 PM): do u have a pic?

38 MAN (03/31/07 4:44:30 PM): yes i am

$39 \operatorname{MAN}(03 / 31 / 07$ 4:44:34 PM): yes i do

40 DEC (03/31/07 4:44:40 PM): then it doesn't matter howold u r, right?

41 DEC (03/31/07 4:44:43 PM): as logn as ur nice

$42 \operatorname{MAN}(03 / 31 / 07$ 4:44:48 PM): no it doesn't

\section{Chat \#4}

1 MAN (07/21/09 4:11:00 PM): hi

2 DEC (07/21/09 4:11:34 PM): hi

3 MAN (07/21/09 4:11:44 PM): 49m/monroe mi

4 DEC (07/21/09 4:11:52 PM): im heather

5 MAN (07/21/09 4:12:27 PM): where ru from heather

6 DEC (07/21/09 4:12:27 PM): $14 \mathrm{f} \mathrm{mi}$

7 DEC (07/21/09 4:12:27 PM): whats ur name?

8 MAN (07/21/09 4:12:40 PM): im mike

9 DEC (07/21/09 4:13:06 PM): i live here now

10 DEC (07/21/09 4:13:10 PM): i just moved here

11 MAN (07/21/09 4:13:14 PM): where in mi

$12 \operatorname{DEC}(07 / 21 / 09$ 4:13:20 PM): oh

13 DEC (07/21/09 4:13:28 PM): like might tell u when i know u better

14 MAN (07/21/09 4:13:45 PM): ok, you like older men heather

15 DEC (07/21/09 4:14:08 PM): ya

16 DEC (07/21/09 4:14:12 PM): they $\mathrm{r}$ ok

17 MAN (07/21/09 4:14:28 PM): what do you like them to do

18 DEC (07/21/09 4:15:02 PM): idk

19 MAN (07/21/09 4:15:16 PM): you like them to kiss you

20 DEC (07/21/09 4:15:52 PM): might b ok

21 MAN (07/21/09 4:16:12 PM): where can i kiss you if you let me

22 DEC (07/21/09 4:16:17 PM): idk

23 DEC (07/21/09 4:16:23 PM): brb cause im babysitting

24 DEC (07/21/09 4:16:32 PM): $n$ he woke up

25 MAN (07/21/09 4:16:37 PM): k

26 DEC (07/21/09 4:19:27 PM): back

27 MAN (07/21/09 4:20:03 PM): would yo ulike me to rub your back

28 DEC (07/21/09 4:20:24 PM): might b kool

29 MAN (07/21/09 4:20:42 PM): i would rub your butt softly fo ryou

30 DEC (07/21/09 4:21:04 PM): really?

31 MAN (07/21/09 4:21:15 PM): would you like that

32 DEC (07/21/09 4:21:21 PM): idk

33 DEC (07/21/09 4:21:26 PM): might b ok

34 MAN (07/21/09 4:21:53 PM): i would pu tmy hand insid eyour panties when i rub it 
35 DEC (07/21/09 4:22:07 PM): k

36 MAN (07/21/09 4:22:30 PM): how about your slit?

37 DEC (07/21/09 4:23:45 PM): idk

38 MAN (07/21/09 4:24:10 PM): i just use my tongue on that

39 DEC (07/21/09 4:24:21 PM): really?

40 MAN (07/21/09 4:24:28 PM): yes

41 DEC (07/21/09 4:24:49 PM): k

42 MAN (07/21/09 4:24:57 PM): would you like that

43 DEC (07/21/09 4:26:15 PM): ya

44 MAN (07/21/09 4:26:36 PM): want me to suck on your slit softly

45 DEC (07/21/09 4:26:59 PM): idk

46 DEC (07/21/09 4:27:06 PM): like do guys like that?

47 MAN (07/21/09 4:27:21 PM): yes we do

\section{Chat \#5}

1 MAN (10/11/08 9:22:26 PM): Hi

2 MAN (10/11/08 9:22:39 PM): R U female

3 MAN (10/11/08 9:22:49 PM):

4 DEC (10/11/08 9:23:03 PM): yes and dont buzz me

5 MAN (10/11/08 9:23:09 PM): ok

6 DEC (10/11/08 9:23:16 PM): whats yer asl

7 MAN (10/11/08 9:23:25 PM): 29 M Detroit

8 MAN (10/11/08 9:23:28 PM): \& urs

9 DEC (10/11/08 9:23:34 PM): 14/f/mi

$10 \operatorname{MAN}(10 / 11 / 08$ 9:23:43 PM): U have cam

11 DEC (10/11/08 9:23:48 PM): no do u

12 MAN (10/11/08 9:23:52 PM): nope

$13 \operatorname{MAN}(10 / 11 / 08$ 9:24:02 PM): where in Mi U live

14 DEC (10/11/08 9:24:09 PM): like western part

15 MAN (10/11/08 9:24:24 PM): what are ur hobbies

16 DEC (10/11/08 9:24:47 PM): hanging out watching tv cheerleading

17 DEC (10/11/08 9:24:50 PM): what bout u?

18 MAN (10/11/08 9:25:09 PM): Drinking, Dating

$19 \operatorname{DEC}(10 / 11 / 08$ 9:25:38 PM): thats cool

20 MAN (10/11/08 9:25:41 PM): How far is Auburn Hills from ur place

21 DEC (10/11/08 9:26:05 PM): u mean how far is detroit?

22 MAN (10/11/08 9:26:20 PM): I stay in Auburn Hills

23 MAN (10/11/08 9:26:28 PM): Where do u stay

24 DEC (10/11/08 9:27:10 PM): i'm like 2 hours from detroit. i dont know where auburn hills is

25 MAN (10/11/08 9:27:24 PM): R U alone

26 DEC (10/11/08 9:27:49 PM): my mom is home but not in this room why?

27 MAN (10/11/08 9:28:12 PM): Can we meet some where

28 DEC (10/11/08 9:28:21 PM): for what

29 MAN (10/11/08 9:28:34 PM): Date

30 DEC (10/11/08 9:28:42 PM): u want 2 date me?

31 MAN (10/11/08 9:28:48 PM): If $U$ dont mind

32 MAN (10/11/08 9:29:01 PM): :-*

33 DEC (10/11/08 9:29:03 PM): i just met $u$

34 DEC (10/11/08 9:29:12 PM): i dont even know yer name

35 MAN (10/11/08 9:29:36 PM): My Name is Mani

Chat \#6

1 MAN (05/30/08 10:12:20 PM): 113?

$2 \operatorname{MAN}(05 / 30 / 08$ 10:12:24 PM): wow..

3 DEC (05/30/08 10:12:29 PM): lol 
4 DEC (05/30/08 10:12:31 PM): asl?

5 MAN (05/30/08 10:12:36 PM): you can still see?

6 DEC (05/30/08 10:12:40 PM): lol

7 MAN (05/30/08 10:12:40 PM): holy fuck

8 DEC (05/30/08 10:12:44 PM): $13 \mathrm{fct}$

9 MAN (05/30/08 10:12:46 PM): 28 m waterbury

10 MAN (05/30/08 10:12:56 PM): what part?

11 DEC (05/30/08 10:13:08 PM): meriden

12 MAN (05/30/08 10:13:21 PM): im in waterbury

13 MAN (05/30/08 10:13:30 PM): why so young in this chat?

14 DEC (05/30/08 10:13:36 PM): bored

15 MAN (05/30/08 10:13:38 PM): alls thats gonna find you is trouble

16 DEC (05/30/08 10:13:39 PM): y $\mathrm{r}$ u here?

17 MAN (05/30/08 10:13:43 PM): n guys like me with no morals

18 DEC (05/30/08 10:13:46 PM): lol

19 DEC (05/30/08 10:13:56 PM): welll thats not boring i guess lol

20 MAN (05/30/08 10:14:04 PM): wanna get kidnapped?

21 MAN (05/30/08 10:14:05 PM): lol

$22 \operatorname{DEC}(05 / 30 / 08$ 10:14:12 PM): lol

23 DEC (05/30/08 10:14:13 PM): sure

$24 \operatorname{MAN}(05 / 30 / 08$ 10:14:19 PM): riiiiight

25 DEC (05/30/08 10:14:24 PM): :

26 DEC (05/30/08 10:14:25 PM): :D

27 MAN (05/30/08 10:14:43 PM): is this chris hanson????

28 MAN (05/30/08 10:14:43 PM): lol

29 DEC (05/30/08 10:14:43 PM): y? do u want to kidnap me?

$30 \operatorname{MAN}(05 / 30 / 08$ 10:14:47 PM): maybe

31 DEC (05/30/08 10:14:55 PM): lol

32 MAN (05/30/08 10:15:11 PM): pics / cam?

33 DEC (05/30/08 10:15:21 PM): i got pix

34 MAN (05/30/08 10:15:35 PM): send file them to me

35 DEC (05/30/08 10:15:45 PM): ur bossy arent $u$ ?

36 MAN (05/30/08 10:15:49 PM): a little

37 MAN (05/30/08 10:16:09 PM): www.myspace.com/MAN

Chat \#7

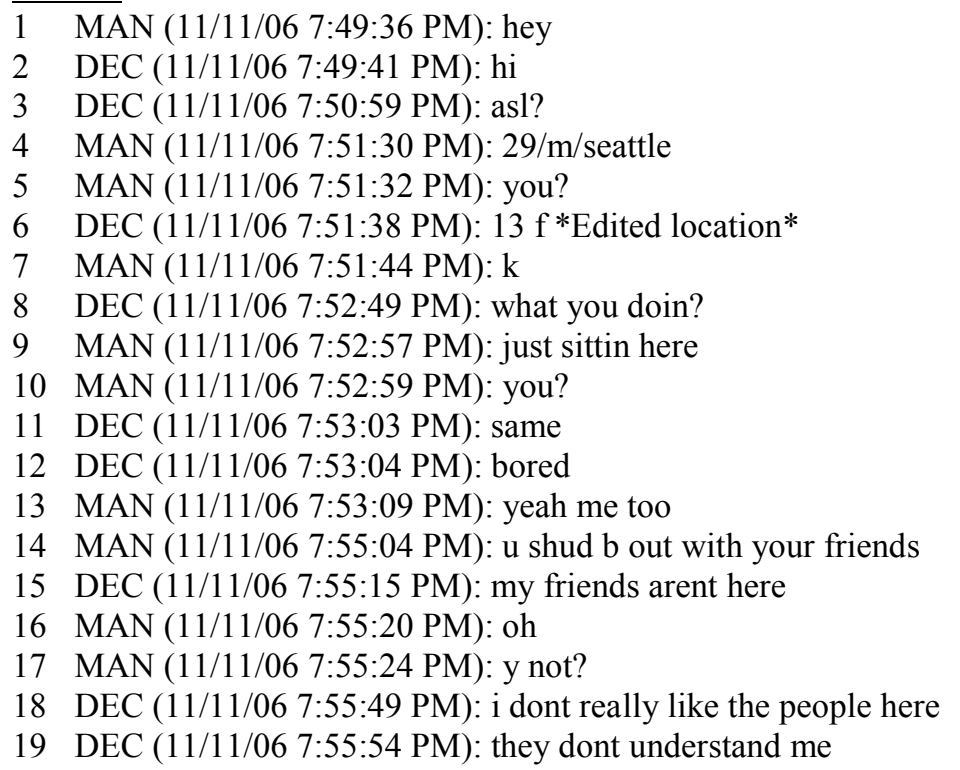


20 MAN (11/11/06 7:56:06 PM): why? what dont they understand?

21 DEC (11/11/06 7:56:35 PM): i do 4h goat raising and really like it

$22 \operatorname{DEC}(11 / 11 / 06$ 7:56:45 PM): and they dont get that

$23 \operatorname{MAN}(11 / 11 / 06$ 7:56:46 PM): thats cool

24 MAN (11/11/06 7:56:59 PM): bet your b/f thinks it cool

25 DEC (11/11/06 7:57:01 PM): i guess i could switch to dog project and be okay but they dont get the goat thing

26 DEC (11/11/06 7:57:04 PM): lol i dont have a bf

27 MAN (11/11/06 7:57:25 PM): y not?

28 DEC (11/11/06 7:57:43 PM): lol like i said the people here dont really get me

$29 \operatorname{MAN}(11 / 11 / 06$ 7:57:51 PM): ohok

30 MAN (11/11/06 7:58:31 PM): sorry

31 DEC (11/11/06 7:59:37 PM): its okay

32 MAN (11/11/06 8:00:19 PM): you look cute

33 DEC (11/11/06 8:00:24 PM): lol tyvm

34 MAN (11/11/06 8:00:25 PM): you shud have a b/f

35 DEC (11/11/06 8:00:29 PM): :(

36 DEC (11/11/06 8:01:04 PM): do you have a pic?

37 MAN (11/11/06 8:01:22 PM): yeah

38 DEC (11/11/06 8:01:54 PM): omg ur cute lol

$39 \operatorname{MAN}(11 / 11 / 06$ 8:02:26 PM): lol

\section{Chat \#8}

1 MAN (09/07/06 5:08:25 PM): hi

2 DEC (09/07/06 5:09:22 PM): hi

3 MAN (09/07/06 5:09:32 PM): hi

4 MAN (09/07/06 5:09:36 PM): how are u?

5 DEC (09/07/06 5:10:09 PM): ok asl

6 MAN (09/07/06 5:10:30 PM): m/ca/oc/21

7 MAN (09/07/06 5:10:32 PM): u?

8 MAN (09/07/06 5:12:11 PM): u cute

9 MAN (09/07/06 5:12:16 PM): where do u live

10 DEC (09/07/06 5:13:25 PM): $12 \mathrm{f}$ cali

11 MAN (09/07/06 5:13:33 PM): where in ca

12 DEC (09/07/06 5:13:49 PM): socal

13 MAN (09/07/06 5:14:01 PM): i live near disneyland

14 DEC (09/07/06 5:14:12 PM): k

15 MAN (09/07/06 5:14:20 PM): u far

16 DEC (09/07/06 5:15:27 PM): huh

17 MAN (09/07/06 5:15:35 PM): ok

18 MAN (09/07/06 5:15:42 PM): do u have cam

19 MAN (09/07/06 5:15:47 PM): or pic

20 DEC (09/07/06 5:16:17 PM): my pic is on my profile

21 MAN (09/07/06 5:16:54 PM): more pic

22 DEC (09/07/06 5:17:33 PM): wheres urs

23 DEC (09/07/06 5:18:35 PM): ur kinda cute

24 MAN (09/07/06 5:18:50 PM): thanks

25 MAN (09/07/06 5:18:57 PM): do u have more pic

$26 \operatorname{MAN}(09 / 07 / 06$ 5:19:39 PM): nice

27 DEC (09/07/06 5:20:18 PM): thx

$28 \operatorname{MAN}(09 / 07 / 06$ 5:20:26 PM):;;)

29 MAN (09/07/06 5:20:33 PM): do u have bf?

30 DEC (09/07/06 5:20:36 PM): no

31 MAN (09/07/06 5:20:43 PM): ok 


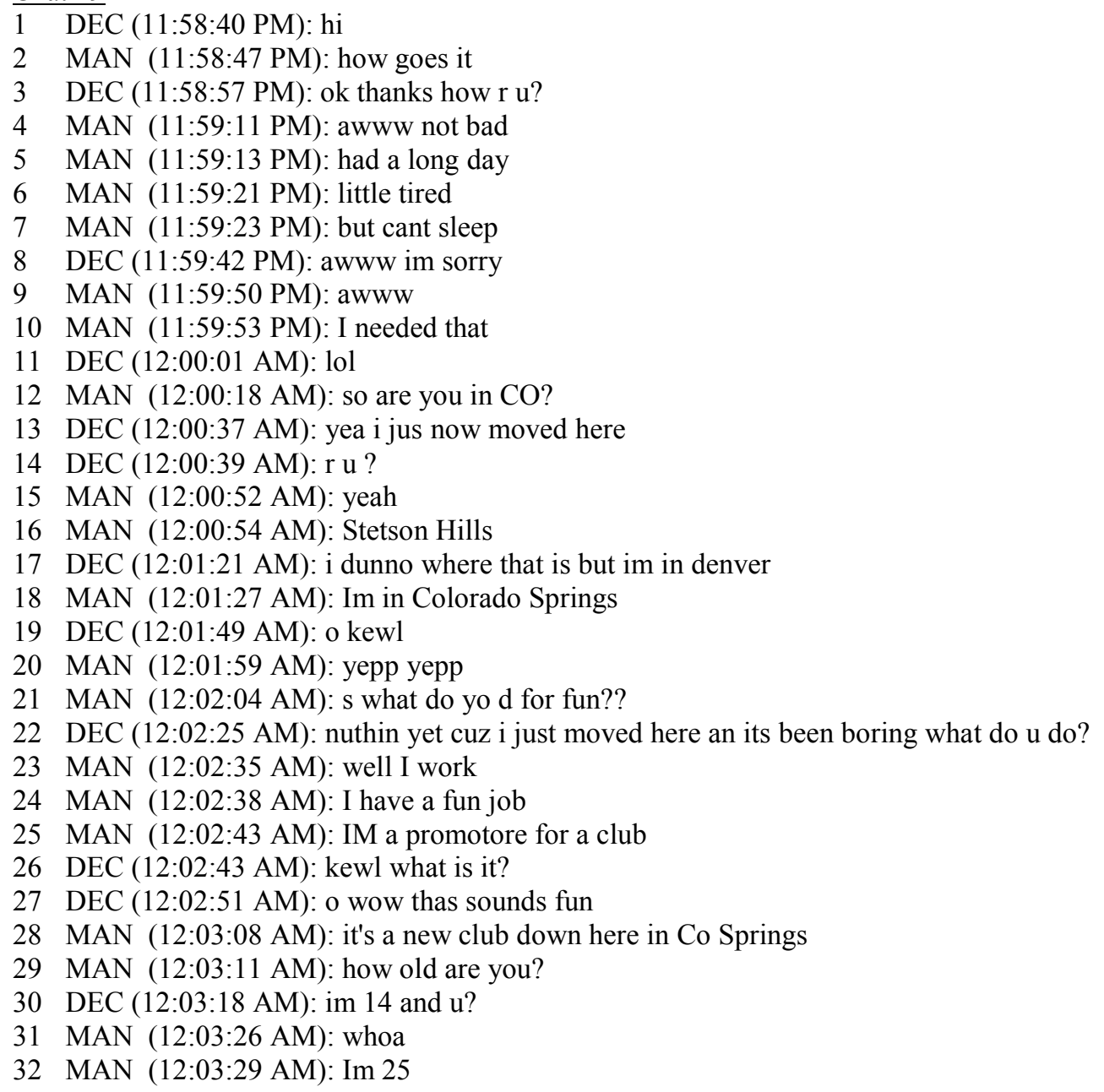

\section{Chat \#10}

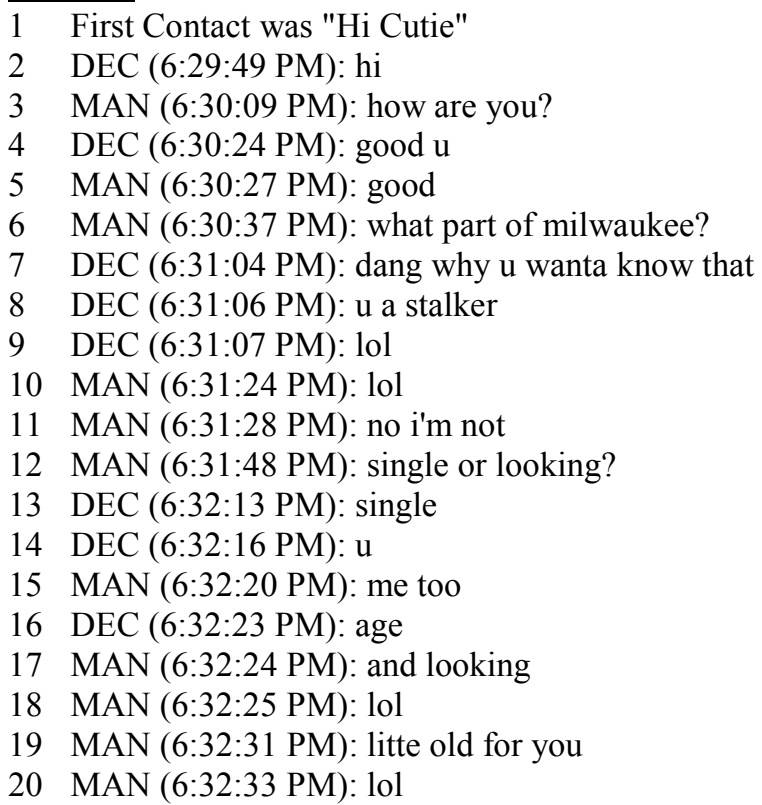


21 DEC (6:32:34 PM): dang

22 DEC (6:32:35 PM): lol

23 MAN (6:32:37 PM): you like older gusy

24 DEC (6:32:39 PM): sure

25 MAN (6:33:00 PM): what do you do for fun?

26 DEC (6:33:13 PM): party

27 DEC (6:33:16 PM): listen to music

28 DEC (6:33:18 PM): u

29 MAN (6:33:34 PM): movies clubs sex hehe

30 DEC (6:33:47 PM): lol kewl

31 DEC (6:33:57 PM): im bettie

32 DEC (6:33:57 PM): u

33 MAN (6:34:00 PM): you like sex too

34 DEC (6:34:13 PM): yes

35 MAN (6:34:37 PM): nice!

36 DEC (6:34:53 PM):

37 MAN (6:34:58 PM): hehe

38 MAN (6:35:14 PM): you had sex to many times yed?

39 DEC (6:35:20 PM): a couple yeah

40 MAN (6:35:39 PM): you like it?

41 DEC (6:35:49 PM): yes

42 MAN (6:36:24 PM): did you ever been lickit?

Chat \#11

1 MAN (08/22/06 12:15:23 AM): hi

2 DEC (08/22/06 12:15:36 AM): hi asl?

3 MAN (08/22/06 12:15:53 AM): 32 m fresno

4 MAN (08/22/06 12:15:53 AM): u?

5 DEC (08/22/06 12:16:12 AM): 13/f/ca, how $\mathrm{r} u$ ?

6 MAN (08/22/06 12:16:18 AM): 13?

7 MAN (08/22/06 12:16:20 AM): wow

8 MAN (08/22/06 12:16:21 AM): really?

9 DEC (08/22/06 12:16:27 AM): ya

10 MAN (08/22/06 12:16:34 AM): ok

11 MAN (08/22/06 12:16:37 AM): got a cam?

$12 \operatorname{DEC}(08 / 22 / 06$ 12:17:08 AM): naw, mom took it and sold it on ebay coz she said i could get in2 trouble

$13 \operatorname{MAN}(08 / 22 / 06$ 12:17:18 AM): lol

14 MAN (08/22/06 12:17:22 AM): any more pics?

15 DEC (08/22/06 12:18:19 AM): 1 more...ill send 18r okies?

16 MAN (08/22/06 12:18:29 AM): ok

17 MAN (08/22/06 12:18:33 AM): mic?

18 DEC $(08 / 22 / 06$ 12:18:45 AM): no

19 DEC (08/22/06 12:19:15 AM): ill send that pic now if $u$ want it

20 MAN (08/22/06 12:19:20 AM): ok

21 MAN (08/22/06 12:20:17 AM): send it

22 MAN (08/22/06 12:20:28 AM):

23 MAN (08/22/06 12:20:36 AM): ooops

24 MAN (08/22/06 12:20:40 AM): so what's on your mind?

25 DEC (08/22/06 12:21:03 AM): nothin much, jus bored lol u?

26 MAN (08/22/06 12:21:11 AM): bored and horny

27 MAN (08/22/06 12:21:12 AM): lol

28 MAN (08/22/06 12:21:19 AM): what $\mathrm{r} u$ wearing?

Chat \#12 
MAN (09/22/06 10:31:18 PM): hi

MAN (09/22/06 10:31:51 PM): your very pretty

DEC (09/22/06 10:32:43 PM): hi and ty

MAN (09/22/06 10:32:56 PM): whats going on tonight

DEC (09/22/06 10:33:05 PM): nm how bout u?

MAN (09/22/06 10:33:21 PM): just sitting here

MAN (09/22/06 10:33:30 PM): what part of ky $r u$ from

DEC (09/22/06 10:33:43 PM): central part, $\mathrm{n} u$ ?

MAN (09/22/06 10:33:52 PM): lexington

10 DEC (09/22/06 10:33:55 PM): kool

11 DEC (09/22/06 10:34:00 PM): what is ur a/s?

12 MAN (09/22/06 10:34:08 PM): im 44/m

13 MAN (09/22/06 10:34:21 PM): i know im way to old

14 DEC (09/22/06 10:34:41 PM): itz ok, we r just talking

15 MAN (09/22/06 10:34:45 PM): good

16 MAN (09/22/06 10:34:52 PM): what would u like to talk about

17 DEC (09/22/06 10:35:10 PM): i dont care, wat do u do in lexinton?

18 MAN (09/22/06 10:35:24 PM): i delive medical supplies

19 MAN (09/22/06 10:35:32 PM): i work thats about all i do

20 MAN (09/22/06 10:35:37 PM): what do you do

21 DEC (09/22/06 10:35:51 PM): hang on a min

22 MAN (09/22/06 10:35:54 PM): ok

$23 \operatorname{MAN}(09 / 22 / 06$ 10:44:19 PM): are you there

24 DEC (09/22/06 10:44:46 PM): yah hang on im srry

25 MAN (09/22/06 10:44:54 PM): ok ill be here

26 DEC (09/22/06 10:52:01 PM): im so srry

27 MAN (09/22/06 10:52:08 PM): thats ok

28 MAN (09/22/06 10:52:15 PM): are you busy

29 DEC (09/22/06 10:52:21 PM): i wasnt, my mom just came up

30 MAN (09/22/06 10:52:47 PM): she doesnt like you in the chat rooms?

31 DEC (09/22/06 10:52:55 PM): haha she doesnt know

32 MAN (09/22/06 10:53:03 PM): thats good

\section{Chat \#13}

1 MAN (07/20/06 5:52:18 PM): sassy how old are you really

2 MAN $(07 / 20 / 065: 52: 28$ PM): 13 or 14

3 DEC (07/20/06 5:53:07 PM): 13 why?

4 MAN (07/20/06 5:53:27 PM): that is what $\mathrm{i}$ thought...yahoo has you marked as 113

5 DEC (07/20/06 5:53:34 PM): i markd it like that

6 DEC (07/20/06 5:53:36 PM): ur asl

7 MAN (07/20/06 5:53:54 PM): i am old enuff to be your father

8 MAN (07/20/06 5:53:56 PM): 35

9 MAN (07/20/06 5:53:59 PM): swm conyers

10 MAN (07/20/06 5:54:26 PM): lol

11 MAN (07/20/06 5:54:44 PM): cheer up

12 MAN (07/20/06 5:54:54 PM): how are you today

13 DEC (07/20/06 5:54:59 PM): my dads in his 40

14 DEC $(07 / 20 / 06$ 5:55:00 PM): k

15 DEC (07/20/06 5:55:21 PM): im ok

16 MAN (07/20/06 5:55:31 PM): ok i was just saying because i have been leterally hit on by a $14 \mathrm{yr}$ old

17 MAN (07/20/06 5:55:41 PM): about 2 weeks ago

18 DEC (07/20/06 5:55:52 PM): and so?

19 MAN (07/20/06 5:56:05 PM): are you looking forward for school to start back

20 DEC (07/20/06 5:56:27 PM): im just startin school down here bu not

21 DEC (07/20/06 5:56:29 PM): but no 
$22 \operatorname{MAN}(07 / 20 / 06$ 5:56:36 PM): why

23 MAN (07/20/06 5:56:42 PM): did you just move back to ga

24 DEC (07/20/06 5:56:47 PM): cuz we movd here last month from columbus ohio

25 MAN (07/20/06 5:57:05 PM): oh ok

26 MAN (07/20/06 5:57:10 PM): welcome to ga then

27 DEC (07/20/06 5:57:17 PM): thnks its HOT down here

28 MAN (07/20/06 5:58:20 PM): i am sorry

29 MAN (07/20/06 5:58:29 PM): which part of georgia you in

30 DEC (07/20/06 5:59:38 PM): columbs area

31 MAN (07/20/06 6:00:06 PM): ahhh

32 MAN (07/20/06 6:00:16 PM): so you are down by fort benning

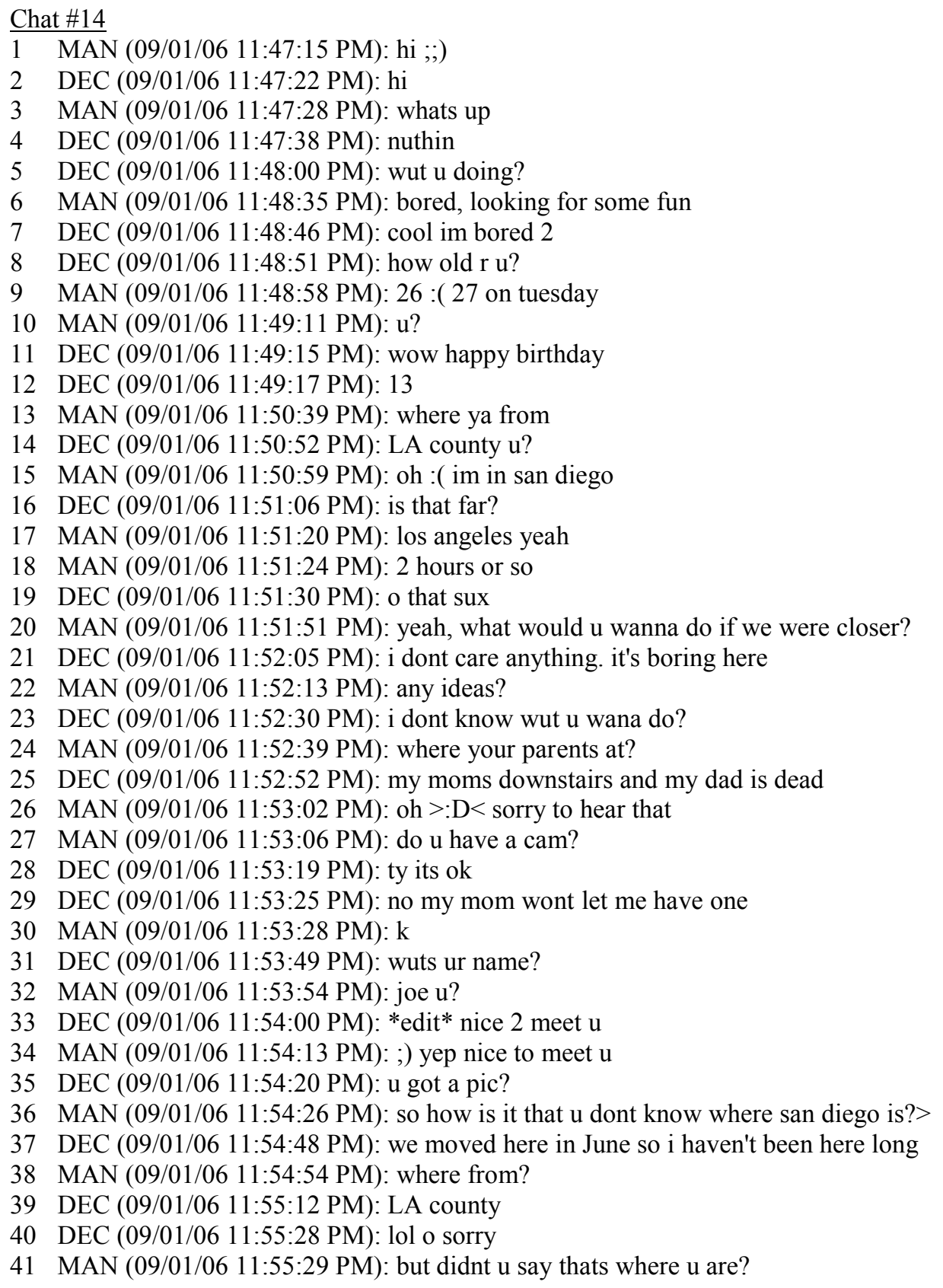


Chat \#15
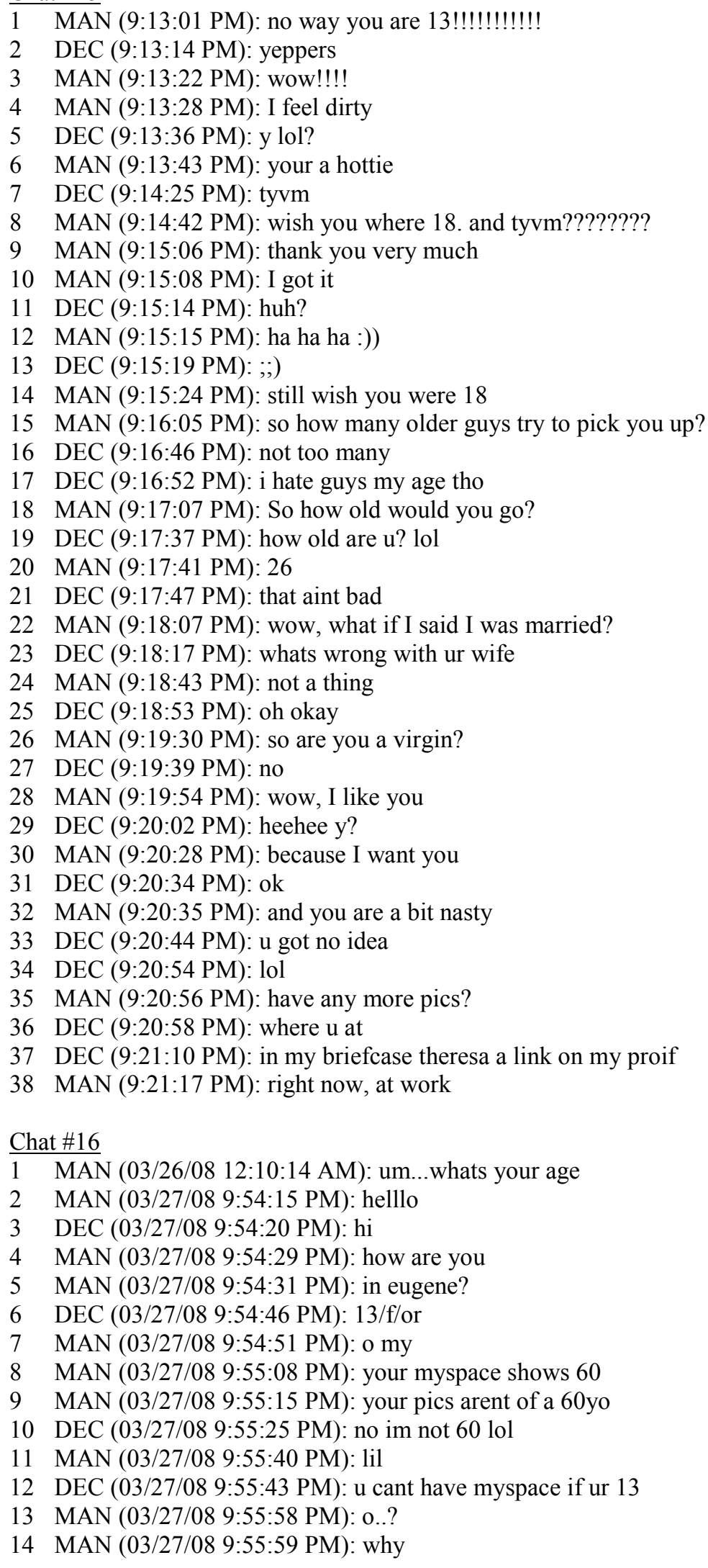
15 MAN (03/27/08 9:56:10 PM): too young?

16 DEC (03/27/08 9:56:18 PM): yeah u gotta b 14

17 DEC (03/27/08 9:56:26 PM): but then they make it private

18 MAN (03/27/08 9:56:26 PM): when will that happen

19 DEC (03/27/08 9:56:40 PM): nov

20 MAN (03/27/08 9:56:49 PM): can i add you

21 DEC (03/27/08 9:56:58 PM): wuts ur asl

22 MAN (03/27/08 9:57:06 PM): 45meugene...

23 DEC (03/27/08 9:57:08 PM): yeah u can

24 MAN (03/27/08 9:57:34 PM): ok

25 MAN (03/27/08 9:58:25 PM): why you an adult romance room

26 DEC (03/27/08 9:58:42 PM): idk im bored

27 MAN (03/27/08 9:58:48 PM): ok...me too

28 MAN (03/27/08 9:58:54 PM): i 11 brb..doing laundry

29 DEC (03/27/08 9:58:59 PM): k

30 MAN (03/27/08 10:03:59 PM): ok

31 DEC (03/27/08 10:04:08 PM): hi wb

32 MAN (03/27/08 10:04:15 PM): thanks

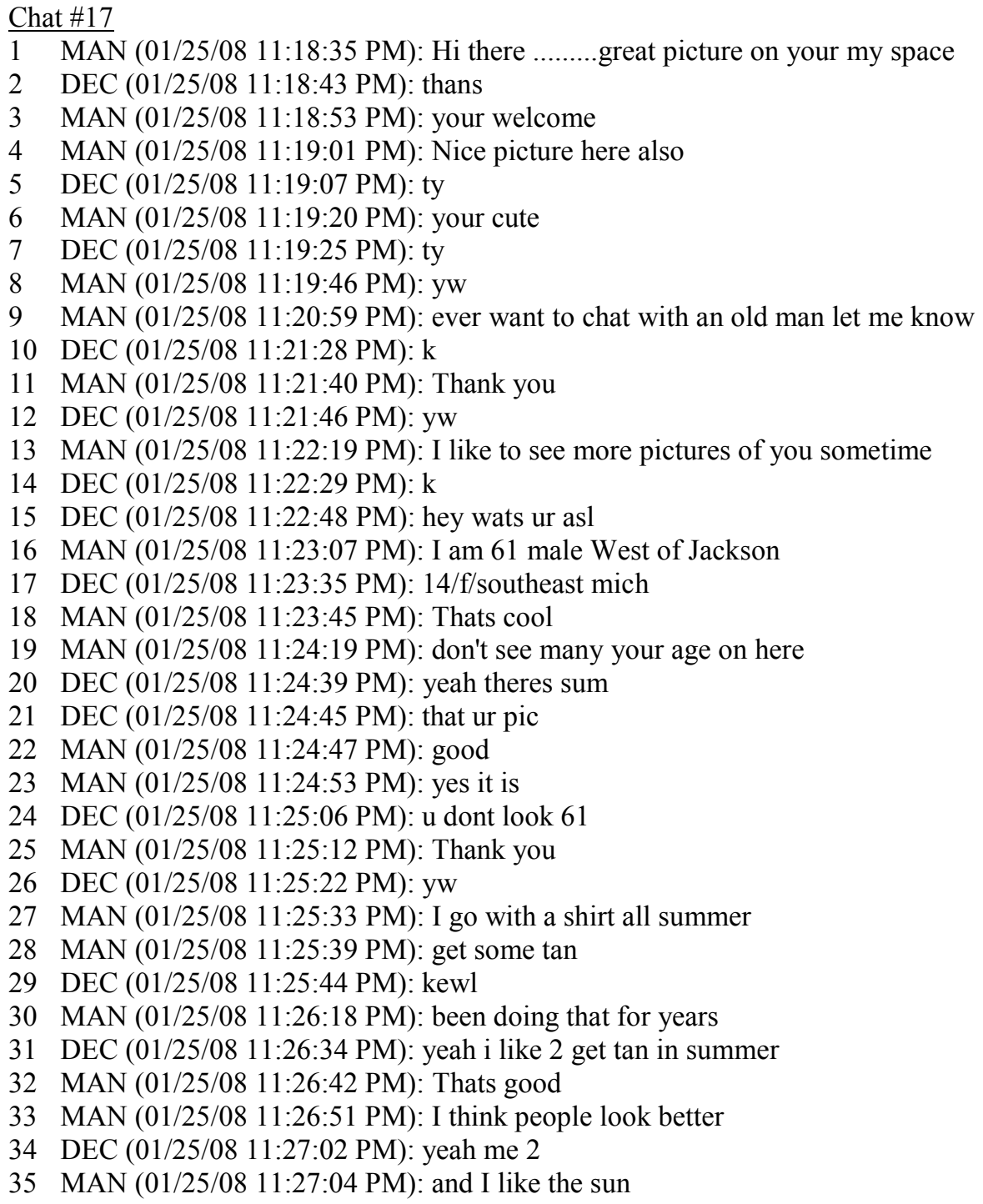




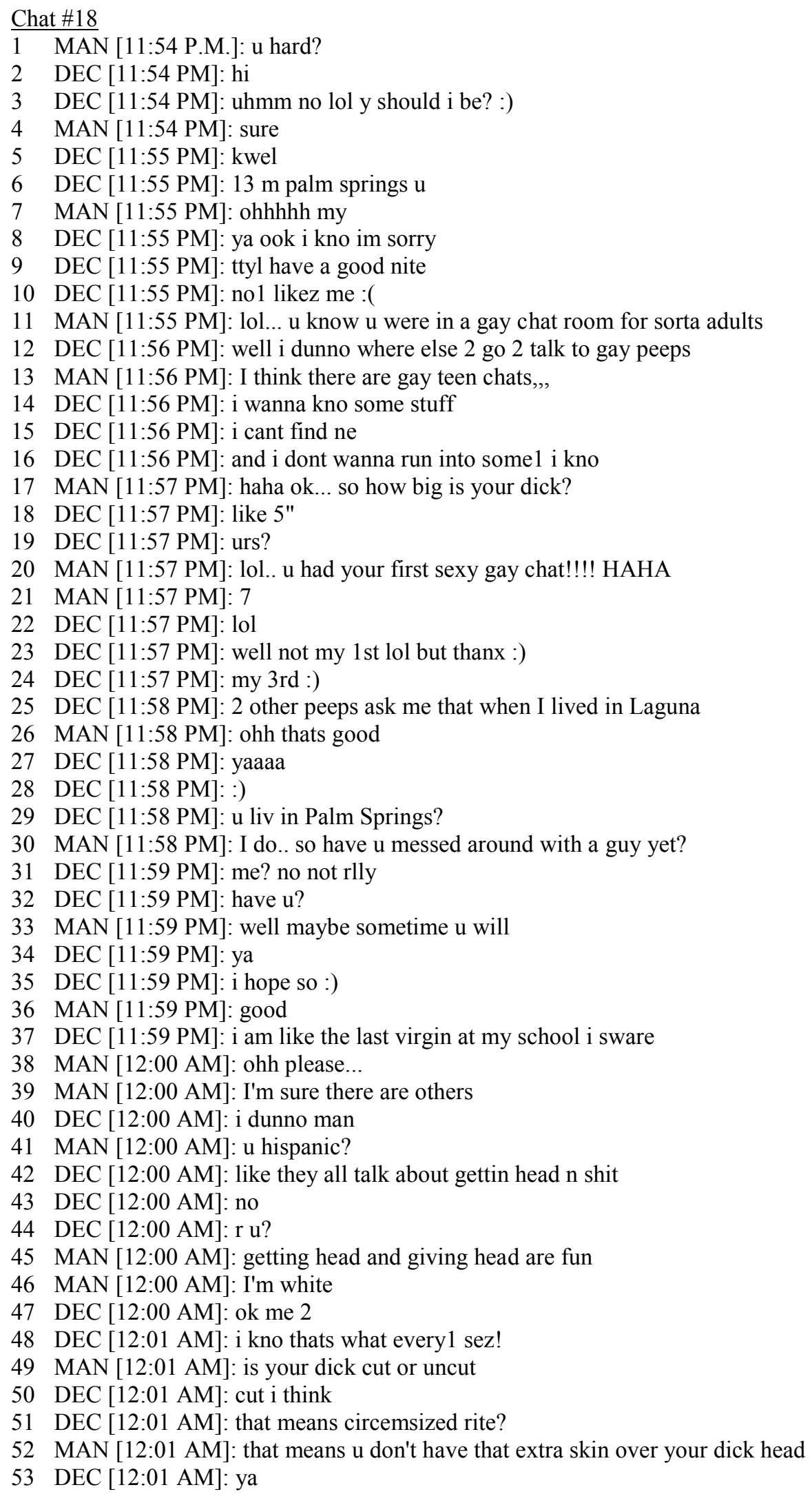




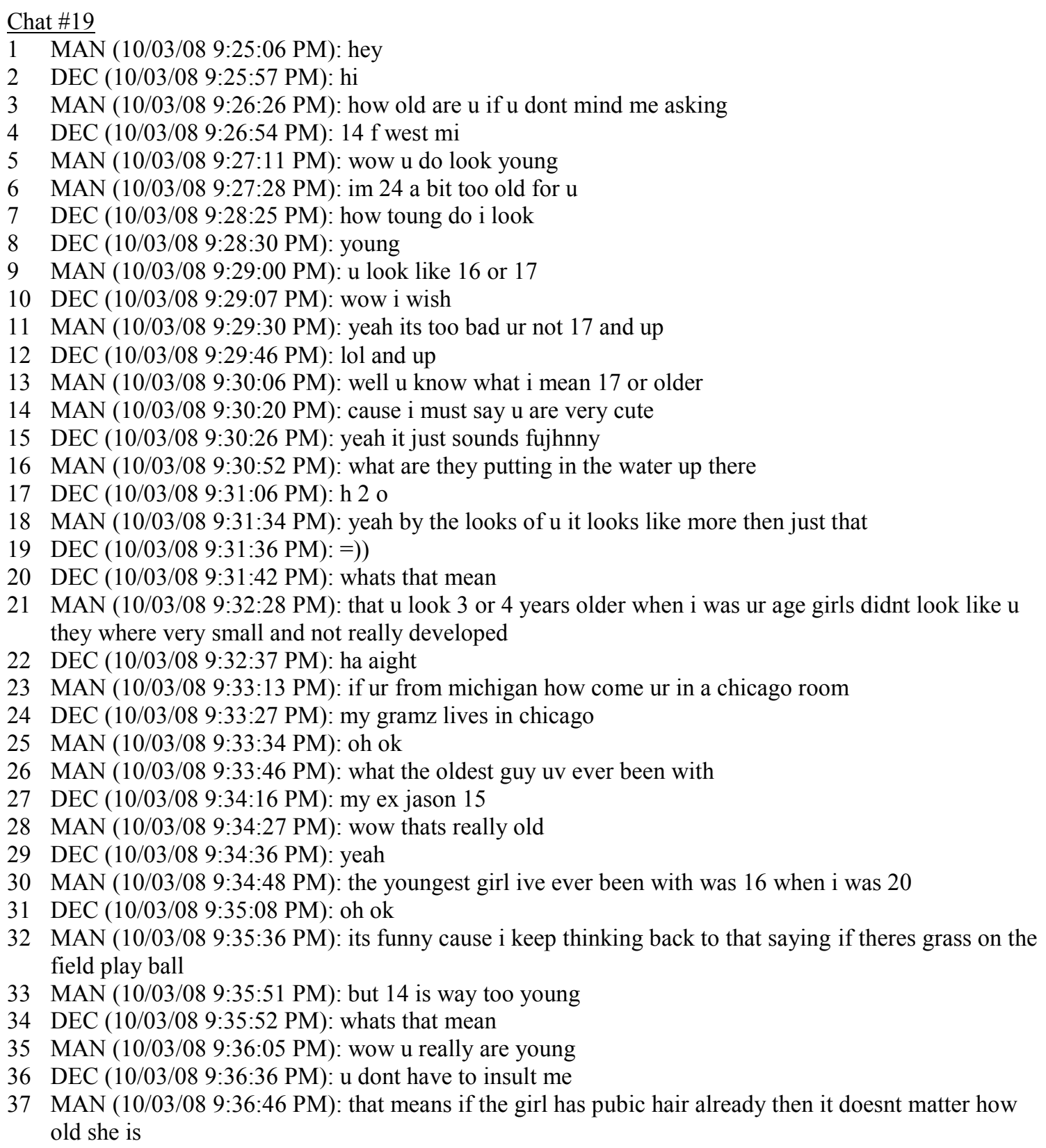

Chat \#20

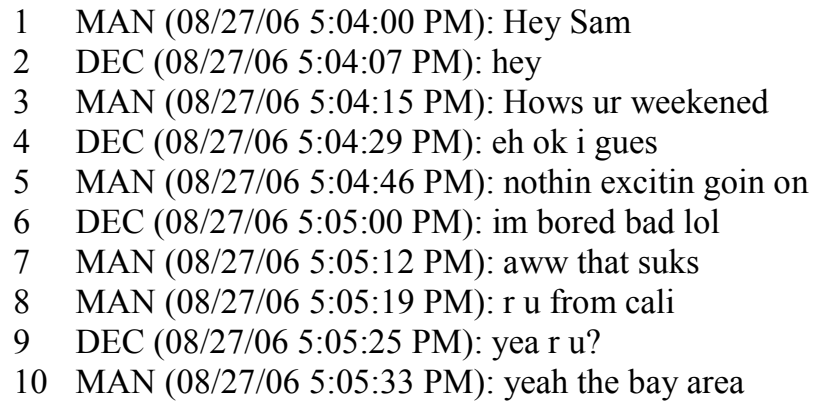


11 MAN (08/27/06 5:06:23 PM): $u$ from the bay

12 DEC (08/27/06 5:06:29 PM): close

13 MAN (08/27/06 5:06:48 PM): tracy stockton

14 DEC (08/27/06 5:06:52 PM): huh

15 MAN (08/27/06 5:07:02 PM): how close

16 DEC (08/27/06 5:07:10 PM): near santa rosa

17 MAN (08/27/06 5:07:24 PM): ahh ok

18 DEC (08/27/06 5:07:33 PM): how old u

19 MAN (08/27/06 5:07:40 PM): $25 \mathrm{U}$

20 DEC (08/27/06 5:07:51 PM): 13

21 MAN (08/27/06 5:08:19 PM): ohh ur almost half my age

22 DEC (08/27/06 5:08:26 PM): lol

$23 \operatorname{MAN}(08 / 27 / 06$ 5:08:30 PM): lol

24 DEC (08/27/06 5:08:38 PM): wha u look like

25 MAN (08/27/06 5:09:01 PM): 5'11 tall work out so kinda muscular

26 DEC (08/27/06 5:09:08 PM): cool

27 DEC (08/27/06 5:09:30 PM): i totaly dig sports lol

28 MAN (08/27/06 5:09:50 PM): cool I played football

29 DEC (08/27/06 5:09:56 PM): that is hot

30 DEC (08/27/06 5:09:58 PM): lol

31 MAN (08/27/06 5:10:18 PM): yeahhhh

32 MAN (08/27/06 5:10:42 PM): lol

33 DEC (08/27/06 5:10:46 PM): lol

34 MAN (08/27/06 5:11:02 PM): so what NFL team do U like

35 DEC (08/27/06 5:11:25 PM): i like basketball and baseball lol

36 MAN (08/27/06 5:11:48 PM): ahh ok which teams

37 DEC (08/27/06 5:11:50 PM): i hav 2 run the timer is beeping on my dinner lol

38 DEC (08/27/06 5:11:56 PM): $u$ gonna $b$ here for a bit

$39 \operatorname{MAN}(08 / 27 / 06$ 5:12:02 PM): yeahh

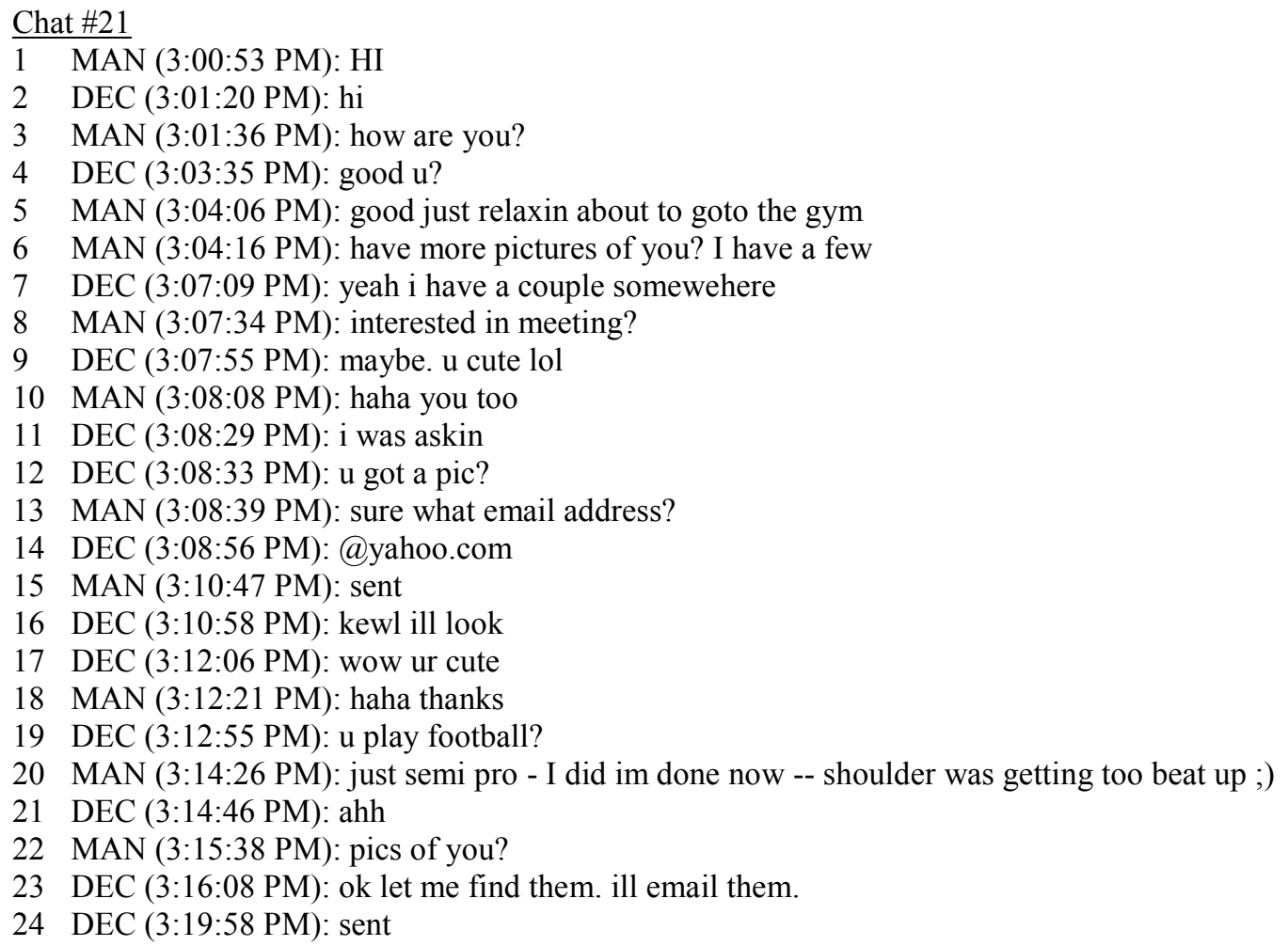


25 MAN (3:20:42 PM): pretty girl ;)

26 MAN (3:20:46 PM): how old?

27 DEC (3:21:24 PM): 13/f/herndon. u?

28 MAN (3:21:43 PM): wow

29 MAN (3:21:45 PM): 34

30 DEC (3:21:58 PM): kewl

31 MAN (3:22:38 PM): haha why like older?

32 DEC (3:22:54 PM): i dont care bout age. ur really cute.

33 MAN (3:23:12 PM): ya what would you like to do? ;)

34 DEC (3:23:26 PM): lol i dont no. what u wanna do?

35 MAN (3:24:04 PM): fool around haha

36 MAN (3:24:12 PM): you?

37 DEC (3:24:49 PM): lol thats kewl. i havent done much though

38 MAN (3:25:04 PM): ya?

Chat \#22

1 MAN (02/23/07 5:48:38 PM): are you off Friday during the day?

2 DEC (02/23/07 5:49:03 PM): umm hi lol

3 DEC (02/23/07 5:50:00 PM): $\mathrm{r} u$ a real person?

4 MAN (02/23/07 5:52:14 PM): yes I am real

5 MAN (02/23/07 5:52:16 PM): sorry

6 MAN (02/23/07 5:52:21 PM): computer freezes sometimes

7 DEC (02/23/07 5:52:27 PM): o lol

8 MAN (02/23/07 5:52:33 PM): lol

9 DEC (02/23/07 5:52:37 PM): i dont think thers any real ppl in ther

10 MAN (02/23/07 5:52:42 PM): What do you think of what I said

11 MAN (02/23/07 5:52:43 PM): lol

12 MAN (02/23/07 5:52:47 PM): tell me about it

13 MAN (02/23/07 5:52:49 PM): mostly bots

14 DEC (02/23/07 5:53:07 PM): so how old $\mathrm{r} u$ ?

15 MAN (02/23/07 5:53:25 PM): 29

16 MAN (02/23/07 5:53:29 PM): and you?

17 DEC (02/23/07 5:53:35 PM): im 14

18 DEC (02/23/07 5:53:42 PM): thers not much on ur prof

$19 \operatorname{MAN}(02 / 23 / 07$ 5:53:47 PM): I know

20 MAN (02/23/07 5:53:55 PM): You are so young

21 MAN (02/23/07 5:53:58 PM): figures

22 MAN (02/23/07 5:54:11 PM): the only girl I get to talk to and we can't hook up

23 DEC (02/23/07 5:54:16 PM): lol

24 DEC (02/23/07 5:54:35 PM): mayb were the only real ppl on yahoo

25 MAN (02/23/07 5:54:44 PM): I know

26 MAN (02/23/07 5:54:46 PM): lol

27 DEC (02/23/07 5:55:15 PM): so were u from?

28 MAN (02/23/07 5:55:28 PM): Princeton,nj

29 MAN (02/23/07 5:55:46 PM): I am working

30 MAN (02/23/07 5:55:56 PM): When are you usually online?

Chat \#23

1 MAN (3:43:03 PM): Hello, this is Nathan from Myspace

2 DEC (3:44:08 PM): asl

3 DEC (3:44:42 PM): have i taked $2 \mathrm{u}$ ?

4 MAN (3:45:11 PM): Yes, you added me recently and replied to two of my e-mails

5 MAN (3:45:19 PM): I am the one with the dog

6 DEC (3:45:50 PM): asl?

7 MAN (3:46:06 PM): age: 23 
8 MAN (3:46:12 PM): sex: male

9 DEC (3:46:24 PM): ok cool

10 MAN (3:46:32 PM): location: Indiana

11 DEC (3:46:46 PM): oh

12 MAN (3:47:05 PM): Not too far away

13 DEC (3:47:17 PM): how far?

14 MAN (3:47:41 PM): Less than fifty miles

15 DEC (3:47:42 PM): oh your da one that asked me about wat $\mathrm{i}$ wuld by if $\mathrm{i}$ had a 200 credit card huh

16 DEC (3:47:44 PM): wow

17 MAN (3:48:00 PM): Yes, have you figured out an answer yet?

18 DEC (3:48:25 PM): bye cloths

19 DEC (3:48:27 PM): lol

20 MAN (3:48:35 PM): Typical girl response

21 MAN (3:49:03 PM): I read your blogs and sounds like you have been having a rough time for a while

22 DEC (3:49:26 PM): ya

23 DEC (3:49:35 PM): $i$ tink it wil be bettr now

24 MAN (3:49:45 PM): You like it better at your dads?

25 DEC (3:50:05 PM): ya

26 DEC (3:50:20 PM): at least he does not call me names or try to steal my bfs

27 MAN (3:50:47 PM): Your mom would try to steal your bfs?!

28 DEC (3:51:19 PM): ya my 20 yo bf that i had for a long time she had sex wit him

29 MAN (3:51:38 PM): Ah, too bad

30 MAN (3:51:47 PM): She must have been a little desperate

31 DEC (3:52:01 PM): i gess

32 MAN (3:52:17 PM): How is school in Ohio?

33 MAN (3:52:32 PM): make many friends yet?

34 DEC (3:53:11 PM): i just really got her sunday

35 DEC (3:53:22 PM): dad says i dont have to start tiill after spring break

36 DEC (3:53:28 PM): so i can get settled

37 MAN (3:53:29 PM): Cool

38 DEC (3:53:33 PM): ya

39 MAN (3:53:48 PM): So are you by yourself all day?

40 DEC (3:54:20 PM): no

41 DEC (3:54:25 PM): my dad gf is here alot

42 DEC (3:54:48 PM): but they $r$ leaving next thur for jamaca

43 MAN (3:55:00 PM): So what happens to you?

\section{Chat \#24}

1 MAN: where u from in MI?

2 DEC: near detroit

3 MAN: same here

4 DEC: age?

5 MAN: 15

6 MAN: u?

7 DEC: 13

8 MAN: if you're looking for somebody older I have an older brother

9 MAN: what do you look like?

10 DEC: I think i have a pic in my pro

11 DEC: you have yahoo?

12 DEC: $\mathrm{XXXX}$ is my yim

13 DEC: I don't have messenger, can you send it to me? my e-mail dragonrage613@yahoo.com

14 DEC: if $u$ have yahoo $u$ ahve yim

15 MAN: I just have yahoo e-mail

16 DEC: im not emailing pix

17 DEC: its easy to get yim 
18 DEC: you can look at my pix on myspace

19 MAN: I'm downloading YIM right now

20 DEC: $\mathrm{k}$

21 MAN: what's ur url on myspace though?

22 DEC: *my myspace url*

23 MAN: you're hot

24 MAN: very hot

25 MAN: you there?

26 MAN: I guess not

27 DEC: omsorry

28 MAN: my mom was calling

29 MAN: that's alright

30 MAN: I think you are very hot

31 DEC: ty!!

32 DEC: u got yim now?

33 MAN: yeah

34 MAN: dragonrage613

*Yahoo Instant Messenger*

35 MAN: (02/24/08 9:50:10 AM): hey there

36 DEC: (02/24/08 9:50:34 AM): that sexyboy613 from teenspot right?

37 MAN: (02/24/08 9:50:38 AM): yep

\section{Chat \#25}

1 MAN (5:50:25 PM): hi

2 DEC (5:53:21 PM): hi

3 MAN (5:54:49 PM):

4 MAN(5:54:51 PM): u there

5 MAN(5:54:54 PM): my name is cory

DEC (5:54:57 PM): hi

DEC (5:55:00 PM): yah im here

DEC (5:55:03 PM): asl

9 MAN(5:55:07 PM): are you from san diego

10 MAN(5:56:06 PM): i'm 21 sweetie i love being with young active people that love to hang out and

11 MAN(5:56:13 PM): i'm from chula vista you

12 MAN (5:56:14 PM): ?

13 DEC (5:56:20 PM): im around *edit*

14 MAN (5:56:25 PM): are you busy tonight would you like to see a movie or something

15 MAN (5:56:30 PM): what is your name sweetie

16 DEC (5:56:32 PM): *kid's name*

17 MAN (5:56:38 PM): it's so beautiful

18 MAN (5:56:41 PM): what about you asl

19 DEC (5:56:43 PM): aww ty

20 DEC (5:56:46 PM): $13 \mathrm{f}$ cali

21 MAN (5:57:42 PM): yea you like the movies and the beach and what music you like

22 DEC (5:58:13 PM): i like all kinds just not stuff like ashlee simpson $n$ her stupid sis

23 MAN (5:58:23 PM): i'm looking for a date to take to the movies tonight would you be interested or just someone to cuddle with in my car at the beach, i can pick you up and i'll pay for everything no worries

24 MAN (5:58:33 PM): i love to kiss and cuddle like teddy bears

25 MAN (5:58:35 PM): lol

26 DEC (5:58:37 PM): lol

27 MAN (5:58:40 PM): u like to

28 MAN (5:58:44 PM): you can never practive enough

29 MAN (5:58:46 PM): lol

30 MAN (5:58:50 PM): >:D<:-* 

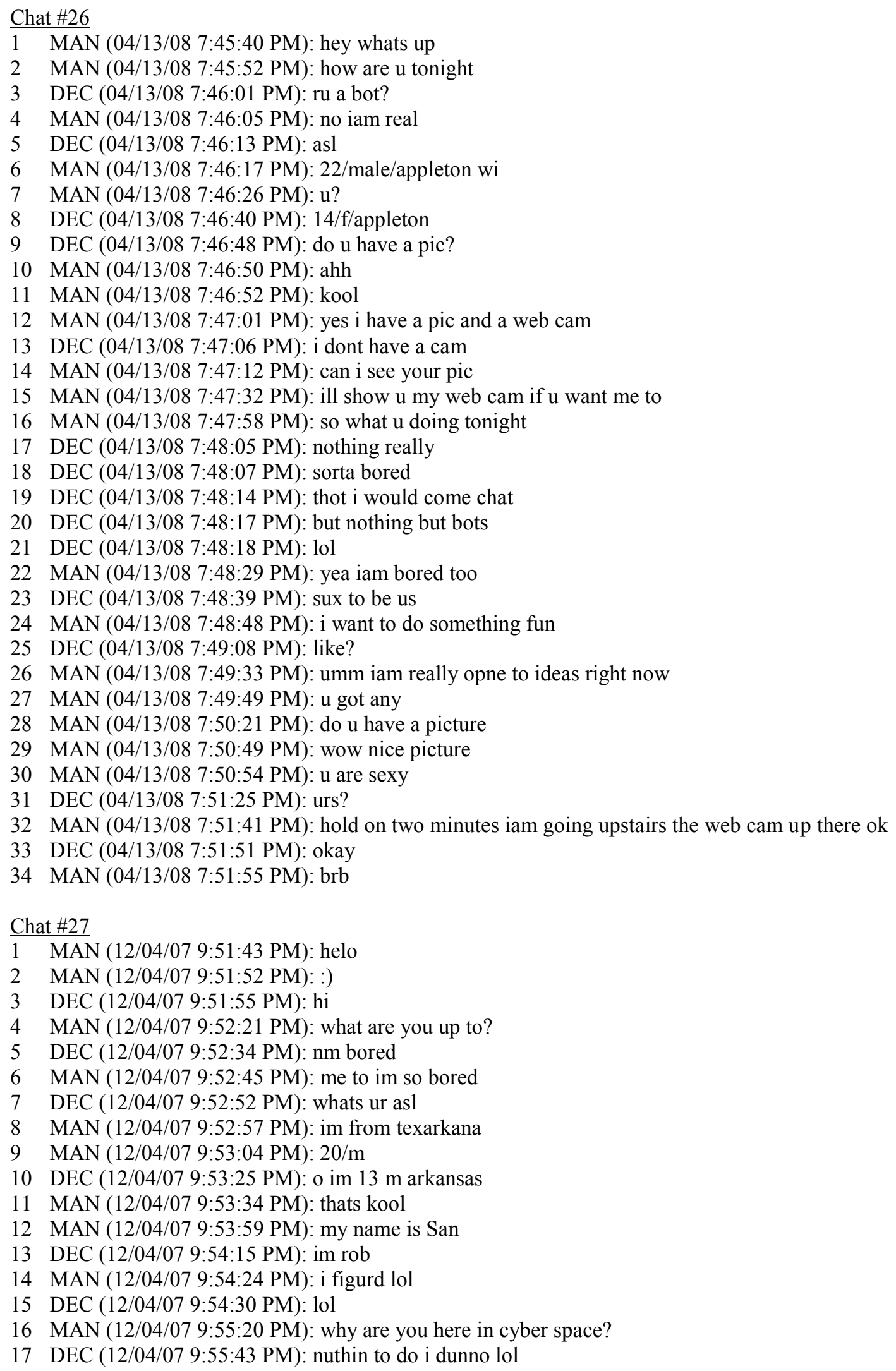
18 MAN (12/04/07 9:55:53 PM): oh

19 MAN (12/04/07 9:56:06 PM): just hanging out here

$20 \operatorname{MAN}(12 / 04 / 07$ 9:56:16 PM): thats kool

21 DEC (12/04/07 9:56:20 PM): yah why $\mathrm{r} u$

22 MAN (12/04/07 9:56:44 PM): im looking for kool new friends

23 DEC (12/04/07 9:56:48 PM): o

$24 \operatorname{MAN}(12 / 04 / 07$ 9:57:37 PM): how is school?

25 DEC (12/04/07 9:58:02 PM): sucks lol

26 MAN (12/04/07 9:58:24 PM): i dont know y i asked that question hehehhe

27 DEC (12/04/07 9:58:37 PM): lol

28 MAN (12/04/07 9:59:28 PM): well few more weeks until christmas

29 MAN (12/04/07 9:59:52 PM): what do you want the most for christmas

30 DEC (12/04/07 9:59:56 PM): ipod

31 MAN (12/04/07 10:00:04 PM): kool

32 MAN (12/04/07 10:00:43 PM): i want a new laptop :">

Chat \#28

1 MAN (12/03/06 9:00:39 PM): hi

2 DEC (12/03/06 9:00:53 PM]: hey :)

3 DEC (12/03/06 9:00:53 PM]: asl

4 MAN (12/03/06 9:01:06 PM]: 40 m metairie u

5 DEC (12/03/06 9:01:18 PM]: 13 f n.o.

6 MAN (12/03/06 9:01:26 PM]: oh ok

7 MAN (12/03/06 9:01:39 PM]: so whats goin on tonight?

8 DEC (12/03/06 9:01:53 PM]: nuttin jus chattn $\mathrm{n}$ stuf

9 DEC (12/03/06 9:01:55 PM]: u

10 MAN (12/03/06 9:02:12 PM]: same here and watchin tv

11 DEC (12/03/06 9:02:43 PM]: cool :)

12 MAN (12/03/06 9:03:07 PM]: i see u from country... where u from?

13 DEC (12/03/06 9:03:37 PM]: used 2 liv clos 2 austin tx

$14 \operatorname{MAN}(12 / 03 / 06$ 9:04:04 PM]: wow ok cool

15 MAN (12/03/06 9:04:11 PM]: so what brings u down here?

$16 \operatorname{DEC}(12 / 03 / 06$ 9:04:12 PM]: lol

17 DEC (12/03/06 9:04:34 PM]: mom is dvorcn dad $n$

18 DEC (12/03/06 9:04:39 PM]: shes $n \mathrm{RN}$ at hospitl

19 DEC (12/03/06 9:04:48 PM]: she come down here to help

20 MAN (12/03/06 9:04:53 PM]: ahh ok

21 MAN (12/03/06 9:05:20 PM]: good money down here and they need all the help they can get alot of them moveed

$22 \operatorname{DEC}(12 / 03 / 06$ 9:06:00 PM]: ya

23 MAN (12/03/06 9:06:27 PM]: so u miss being ocver there

24 DEC (12/03/06 9:07:04 PM]: i miss my dad n bro ya

25 MAN (12/03/06 9:07:40 PM]: well thats undersatndable

26 MAN (12/03/06 9:08:22 PM]: its alot different huh

27 DEC (12/03/06 9:08:40 PM]: ya

28 DEC (12/03/06 9:08:47 PM]: $\mathrm{n}$ i dont gotta lotta frinds

29 MAN (12/03/06 9:09:19 PM]: yeah they do take a while to make and stuff

$30 \operatorname{DEC}(12 / 03 / 06$ 9:09:39 PM]: ya

31 MAN (12/03/06 9:10:12 PM]: so how long have u been down here?

32 DEC (12/03/06 9:11:01 PM]: almos year

33 MAN (12/03/06 9:11:22 PM]: ahh and $u$ dont have to many freids yet

$34 \operatorname{DEC}(12 / 03 / 06$ 9:12:30 PM]: jus 1 angie

35 DEC (12/03/06 9:12:34 PM]: every1 els thinks im stupid

36 DEC (12/03/06 9:12:38 PM]: caus im diffrent

37 MAN (12/03/06 9:13:04 PM]: hmmm well different is good sometimes :) 
38 DEC (12/03/06 9:13:21 PM]: lol i gues

39 MAN (12/03/06 9:13:45 PM]: it is well most people dont understand country type people down here

40 DEC (12/03/06 9:14:07 PM]: ya

41 MAN (12/03/06 9:14:41 PM]: so what school u go to?

42 DEC (12/03/06 9:15:14 PM]: its christin sckool lol i dunno u that good yet

43 MAN (12/03/06 9:15:28 PM]: oh ok no problem

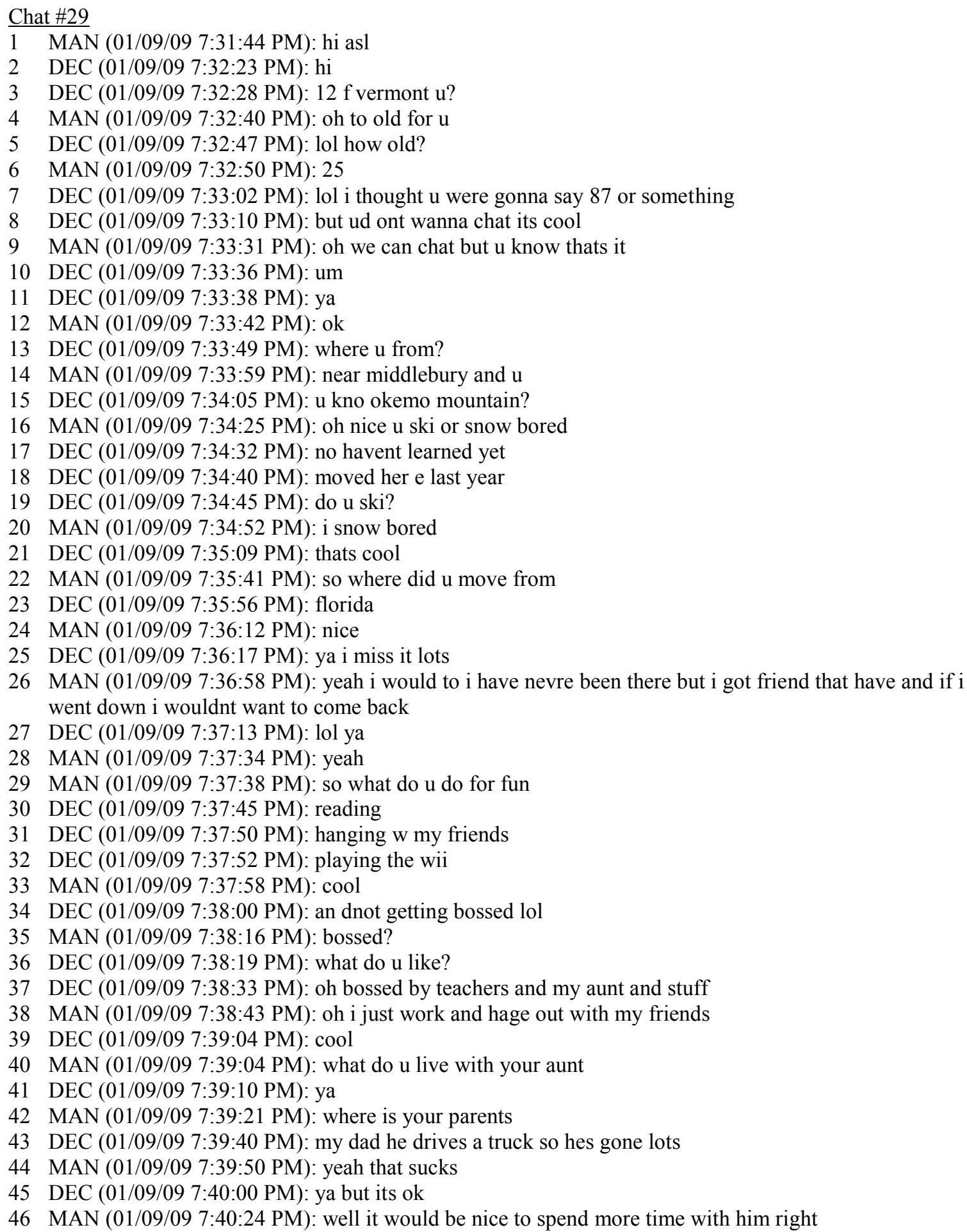




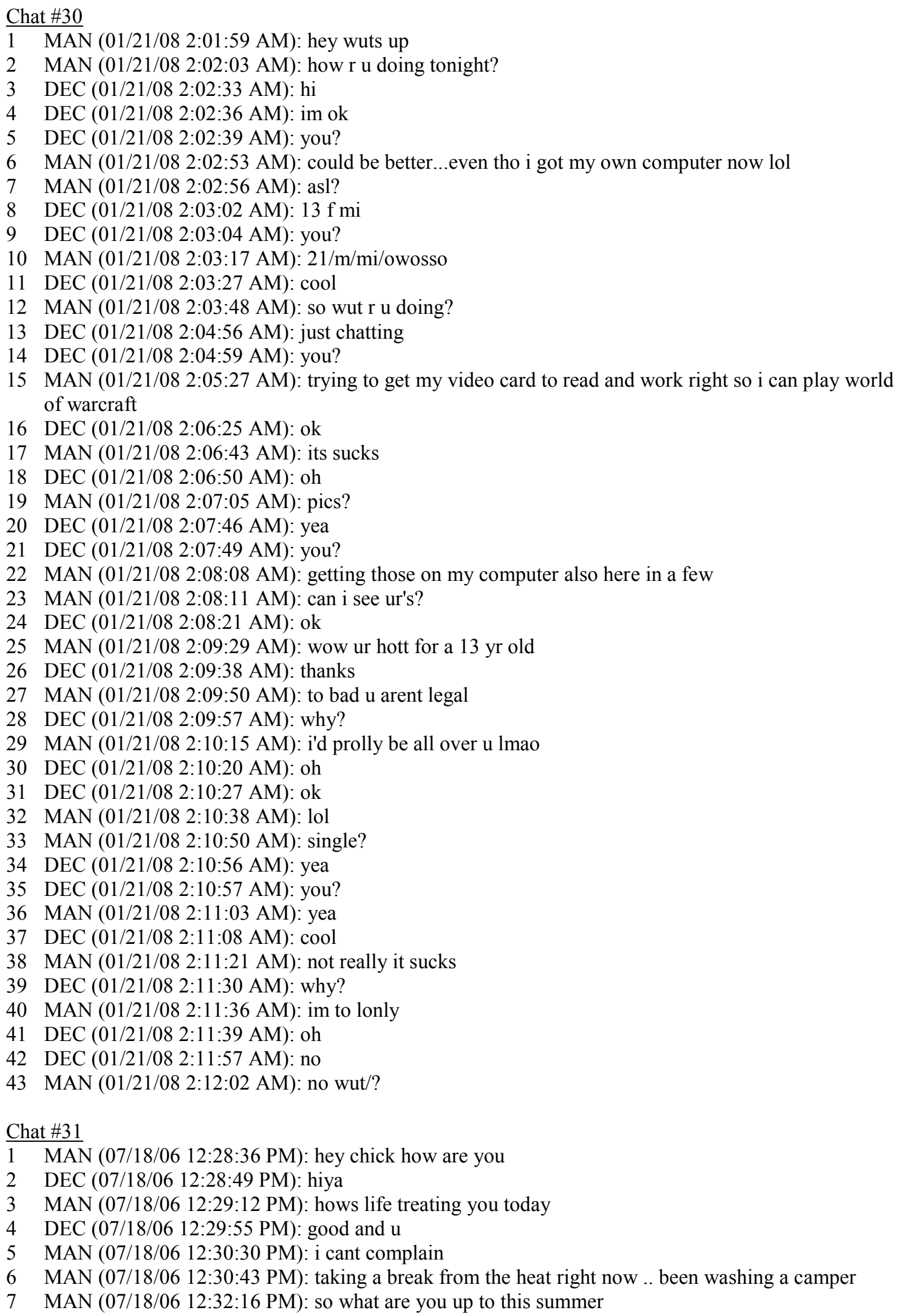




\section{CYBERGROOMING}

12 DEC (07/18/06 12:35:18 PM): it says there is no page

13 DEC (07/18/06 12:35:30 PM): Sorry, the page you requested was not found

14 MAN (07/18/06 12:35:48 PM): ohok .. well im seeking some naughty fun ..

15 MAN (07/18/06 12:36:23 PM): dont know if you are up for that kinda thing or not ... .

16 DEC (07/18/06 12:36:26 PM): i wanna see

17 MAN (07/18/06 12:36:37 PM): see what?

18 DEC (07/18/06 12:36:55 PM): $\mathrm{u}$ in georgia 2

19 MAN (07/18/06 12:37:07 PM): yes i live down near macon .. how bout you?

20 DEC (07/18/06 12:37:22 PM): yeah i am 2

21 DEC (07/18/06 12:37:30 PM): im in harris county

22 MAN $(07 / 18 / 06$ 12:37:35 PM): where is that ?

23 DEC (07/18/06 12:38:13 PM): haha everyone says that

$24 \operatorname{MAN}(07 / 18 / 06$ 12:39:37 PM): you not that far .. cool

25 DEC (07/18/06 12:39:44 PM): no im not

26 MAN (07/18/06 12:40:53 PM): so you like naughty stuff?

27 DEC (07/18/06 12:41:05 PM): yeah

28 MAN (07/18/06 12:41:16 PM): what all have you dont if you dont mind me asking?

29 DEC (07/18/06 12:41:22 PM): irl, not cyber

30 MAN (07/18/06 12:41:44 PM): sorry ... .. what all have you done .. ?

31 DEC (07/18/06 12:42:32 PM): ive like gone 2 third base

32 DEC (07/18/06 12:42:39 PM): and oral

33 MAN (07/18/06 12:42:47 PM): you like oral?

34 DEC (07/18/06 12:43:00 PM): yeah

35 DEC (07/18/06 12:43:14 PM): oh im angel btw.

$36 \operatorname{MAN}(07 / 18 / 06$ 12:43:41 PM): alrighty

37 MAN (07/18/06 12:43:58 PM): can you deepthroat?

$38 \operatorname{DEC}(07 / 18 / 06$ 12:44:36 PM): whats that

39 MAN (07/18/06 12:44:53 PM): might have to show you later :D

\section{Chat \#32}

1 MAN (9:11:12 PM): hi aslr

2 MAN (9:11:13 PM): wanna chat

3 DEC (9:13:18 PM): idk wut $u$ want 2 chat about

4 DEC (9:13:21 PM): asl plz

5 MAN (9:13:24 PM): anything

6 MAN (9:13:26 PM): $22 \mathrm{~m}$ wisc

7 MAN (9:13:26 PM): you

8 DEC (9:13:35 PM): $14 \mathrm{f}$ wi

$9 \operatorname{MAN}(9: 13: 40 \mathrm{PM})$ : where in wi

10 DEC (9:13:55 PM): like kinda close to mil

11 MAN (9:14:00 PM): really

12 MAN (9:14:04 PM): i live milw too

13 DEC (9:15:15 PM): kewl

14 MAN (9:15:22 PM): yea

15 MAN (9:15:24 PM): how tall $\mathrm{u}$ are

16 DEC (9:16:08 PM): 5-4

17 MAN (9:16:24 PM): nice

18 MAN (9:16:25 PM): im 6'4

19 DEC (9:16:42 PM): wow u r tall

20 MAN (9:16:45 PM): yea

21 MAN (9:17:41 PM): what do ya do for fun 
22 DEC (9:18:11 PM): idk all kinds of things

23 DEC (9:18:16 PM): hang out with my friends

24 DEC (9:18:20 PM): shopping

25 DEC (9:18:26 PM): swimming in teh summer

26 MAN (9:18:35 PM): fun

27 DEC (9:19:06 PM): wut do u like 2 do

28 MAN (9:19:33 PM): party,drinks,play pool, darts, sports, fk fk lol,anything fun

29 DEC (9:20:53 PM): wuts fk fk?

$30 \operatorname{MAN}(9: 21: 01 \mathrm{PM})$ : fuck lol

31 DEC (9:21:05 PM): o hehe

$32 \operatorname{MAN}(9: 21: 08$ PM): heh

33 MAN (9:21:39 PM): do u wear a thong or panties or none

34 DEC (9:21:49 PM): panties

35 MAN (9:21:55 PM): kewl

\section{Chat \#33}

1 MAN (11/27/07 11:56:29 PM): heya

2 DEC (11/28/07 12:01:17 AM): hey

3 DEC (11/28/07 12:01:19 AM): whats up

4 MAN (11/28/07 12:01:24 AM): not a whole lot u

5 DEC (11/28/07 12:01:31 AM): same, hmwk

6 DEC (11/28/07 12:01:40 AM): suppperrrr bored

7 MAN (11/28/07 12:01:43 AM): asl?

8 DEC (11/28/07 12:02:18 AM): 14/f/ar

9 DEC (11/28/07 12:02:21 AM): what about u

$10 \operatorname{MAN}(11 / 28 / 07$ 12:02:26 AM): $28 \mathrm{~m} \mathrm{ft} \mathrm{smith}$

11 DEC (11/28/07 12:02:31 AM): kewl

$12 \operatorname{MAN}(11 / 28 / 07$ 12:02:47 AM): where bouts in ark $u$ at

$13 \operatorname{DEC}(11 / 28 / 07$ 12:04:04 AM): ooo on that one spot on the map

14 DEC (11/28/07 12:04:06 AM): u know :)

15 MAN (11/28/07 12:04:15 AM): ok

$16 \operatorname{DEC}(11 / 28 / 07$ 12:04:21 AM): so whats up?

17 MAN (11/28/07 12:04:32 AM): just bored lookin for alittle action tonite

18 DEC (11/28/07 12:04:49 AM): kewl like chattin and all that?

19 MAN (11/28/07 12:05:07 AM): naw like make out and/or sex

20 DEC (11/28/07 12:05:17 AM): ooo i c

$21 \operatorname{MAN}(11 / 28 / 07$ 12:05:22 AM): yep

22 DEC (11/28/07 12:05:27 AM): u meet up with ppl online for that?

23 DEC (11/28/07 12:05:33 AM): err with ppl from online

$24 \operatorname{MAN}(11 / 28 / 07$ 12:05:37 AM): sometimes

25 MAN (11/28/07 12:05:45 AM): its been a while

26 DEC (11/28/07 12:05:54 AM): kewl i nvr did that before

$27 \operatorname{MAN}(11 / 28 / 07$ 12:05:58 AM): its fun

28 DEC (11/28/07 12:06:08 AM): like what kinda ppl u meet?

$29 \operatorname{MAN}(11 / 28 / 07$ 12:06:16 AM): varies

30 MAN (11/28/07 12:06:35 AM): only meet women

31 MAN (11/28/07 12:06:42 AM): but the age varies

32 DEC (11/28/07 12:07:30 AM): lol u mean u get with like 80 yo women?

33 MAN (11/28/07 12:07:50 AM): lol ok i have my limits

34 DEC (11/28/07 12:07:56 AM): lolz

35 MAN (11/28/07 12:08:00 AM): no one over 40 no one under 14

36 DEC (11/28/07 12:08:40 AM): kewl

37 DEC (11/28/07 12:09:02 AM): wow u sound brave lolz like whats been the oldest and youngest?

$38 \operatorname{MAN}(11 / 28 / 07$ 12:09:13 AM): 41 and 14

$39 \operatorname{DEC}(11 / 28 / 07$ 12:09:24 AM): hey kewl like me 
40 DEC (11/28/07 12:09:30 AM): err the 14

41 DEC (11/28/07 12:09:33 AM): im so not 41

42 DEC (11/28/07 12:09:34 AM): yuck

43 MAN (11/28/07 12:09:34 AM): yeh

44 DEC (11/28/07 12:09:35 AM): lolz

$45 \operatorname{MAN}(11 / 28 / 07$ 12:09:38 AM): ha

Chat \#34

1 MAN (5:57:52 PM): hi

2 DEC (5:59:16 PM): hi asl

3 MAN (5:59:41 PM): $23 \mathrm{~m} \mathrm{fl}$

4 DEC (5:59:45 PM): $14 \mathrm{f} \mathrm{fl}$

5 MAN (6:00:13 PM): cool so how u doin

6 DEC (6:00:17 PM): fine u

7 MAN (6:00:35 PM): fine also

8 DEC (6:00:39 PM): brb

9 MAN (6:00:47 PM): ok

10 DEC (6:01:28 PM): k

11 MAN (6:02:07 PM): so u have myspace also

12 DEC (6:02:13 PM): ya

13 MAN (6:02:29 PM): u dont look 84 lol

14 DEC (6:02:35 PM): lol im 14

15 MAN (6:03:12 PM): i know u told me lol

16 DEC (6:03:40 PM): whats urs

17 MAN (6:04:26 PM): http://www.myspace.com/MAN

18 DEC (6:04:48 PM): u r cute

$19 \operatorname{MAN}(6: 05: 16 \mathrm{PM})$ : thanks

20 MAN (6:05:59 PM): u a cuttie also

21 DEC (6:06:15 PM): thanx u can add me if $u$ want

22 MAN (6:07:05 PM): cool do want me too

23 DEC (6:07:10 PM): ya

24 MAN (6:08:10 PM): so u still in school

25 DEC (6:08:15 PM): ya

26 MAN (6:08:28 PM): cool wat grade

27 DEC (6:09:20 PM): 8th

28 MAN (6:09:54 PM): ok u can add me if u want also

29 DEC (6:10:00 PM): kewl

30 MAN (6:11:19 PM): wat u mean by this gurl "try me on for size."

31 DEC (6:11:34 PM): lol $i$ heard it and thot it sounded kewl

32 MAN (6:11:59 PM): ok

33 MAN (6:12:23 PM): and this "anybody who thinks they can handle me. seriously. you think you got what it takes?"

34 MAN (6:12:39 PM): i like that lol

35 DEC (6:12:44 PM): lol

36 MAN (6:13:28 PM): wat u mean about that uh?? Lol

\section{Chat \#35}

\footnotetext{
MAN (08/03/07 8:35:02 PM): hi north ga swm here MAN (08/03/07 8:35:11 PM): r u real

DEC (08/03/07 8:35:18 PM): yea lol

DEC (08/03/07 8:35:21 PM): r u?

MAN (08/03/07 8:35:36 PM): oh kool been too many bots on here

MAN (08/03/07 8:35:42 PM): yes im real thanks

MAN (08/03/07 8:36:18 PM): so what part of ga $r u$ in

8 MAN (08/03/07 8:36:21 PM): north ga here
} 
9 DEC (08/03/07 8:36:33 PM): um im kinda in the middle lol

10 MAN (08/03/07 8:36:49 PM): good place to be sometimes lol

11 MAN (08/03/07 8:37:13 PM): so warner robbins area

12 DEC (08/03/07 8:37:23 PM): kinda

13 MAN (08/03/07 8:37:24 PM): is that thwe middle

14 DEC (08/03/07 8:37:30 PM): lol

15 MAN (08/03/07 8:37:32 PM): hell i dont no

16 DEC (08/03/07 8:37:36 PM): how old $\mathrm{r} u$

17 MAN (08/03/07 8:37:43 PM): 35

18 DEC (08/03/07 8:37:56 PM): o lol

19 DEC (08/03/07 8:38:03 PM): im 13

20 MAN (08/03/07 8:38:14 PM): oh wow

21 DEC (08/03/07 8:38:23 PM): wow wat

22 MAN (08/03/07 8:38:26 PM): i couldnt see profiles so i didnt no

23 MAN (08/03/07 8:38:35 PM): i meant oh wow

24 DEC (08/03/07 8:38:43 PM): oh wow wat

25 MAN (08/03/07 8:38:52 PM): oh well ur hott in that pic

26 DEC (08/03/07 8:39:01 PM): ty

27 MAN (08/03/07 8:39:05 PM): yw

28 DEC (08/03/07 8:39:09 PM): do u got a pic?

$29 \operatorname{MAN}(08 / 03 / 07$ 8:39:30 PM): is it not in the window like urs

30 MAN (08/03/07 8:39:46 PM): yahoo screws up so much mine hardly ever shows

31 DEC (08/03/07 8:39:54 PM): o i gotta clik it

32 DEC (08/03/07 8:40:05 PM): ohhh lol

33 DEC (08/03/07 8:40:12 PM): wow

34 MAN (08/03/07 8:40:14 PM): :">

35 MAN (08/03/07 8:40:22 PM): bad?

\section{Chat \#36}

1 *first lines (Hey there, how are you)

2 DEC (1:11:26 AM): 124 now

3 DEC (1:11:29 AM): why?

4 DEC (1:11:31 AM): ur asl

5 MAN (1:11:53 AM): Just wondering.

6 MAN (1:11:57 AM): 22 m Arizona

7 DEC (1:12:13 AM): hi

8 DEC (1:12:20 AM): ill b 13 on halloween

9 MAN (1:12:42 AM): Wow that is kind of cool to have a birthday then.

10 DEC (1:13:25 AM): i kno $n$ i lovvvvvvvvvvve it

$11 \operatorname{MAN}(1: 13: 35$ AM): :)

12 DEC (1:14:01 AM): i get a big party every yr

$13 \operatorname{MAN}(1: 14: 22 \mathrm{AM})$ : That's awesome

14 DEC (1:14:28 AM): im chrissy

15 DEC (1:14:31 AM): ur name?

16 MAN (1:14:35 AM): Jed

17 DEC (1:14:42 AM): hi jed

18 MAN (1:15:08 AM): I am just sitting here naked.

19 DEC (1:15:19 AM): wont that hurt the chair

$20 \operatorname{MAN}(1: 15: 35 \mathrm{AM})$ : No it will be fine.

21 DEC (1:15:45 AM): oh ok

22 DEC (1:16:00 AM): do u always sit ther naked?

23 MAN (1:16:11 AM): Just sometimes.

24 DEC (1:16:30 AM): oh ok

25 DEC (1:16:34 AM): interstin

26 MAN (1:16:42 AM): Oh yes 
27 MAN (1:16:49 AM): :)

28 DEC (1:17:08 AM): wht else $u$ do besids that

29 DEC (1:17:09 AM): lol

30 MAN (1:17:19 AM): :">

31 MAN (1:17:36 AM): I don't know, would you like to see?

32 DEC (1:17:59 AM): ok

33 MAN (1:18:17 AM): :">

34 DEC (1:20:05 AM): nice

35 MAN (1:20:06 AM): :">

36 DEC (1:20:10 AM): nice

37 MAN (1:20:13 AM): Thank you.

38 MAN (1:20:15 AM): You like it?

39 DEC (1:20:42 AM): itsd ok

40 MAN (1:21:19 AM): Would you like to see it in person?

41 DEC (1:21:27 AM): mayb

42 DEC (1:21:43 AM): but i live wit my aunti

43 DEC (1:21:52 AM): but shes goin outa town in a cople of weeks tho

44 MAN (1:22:04 AM): That's cool

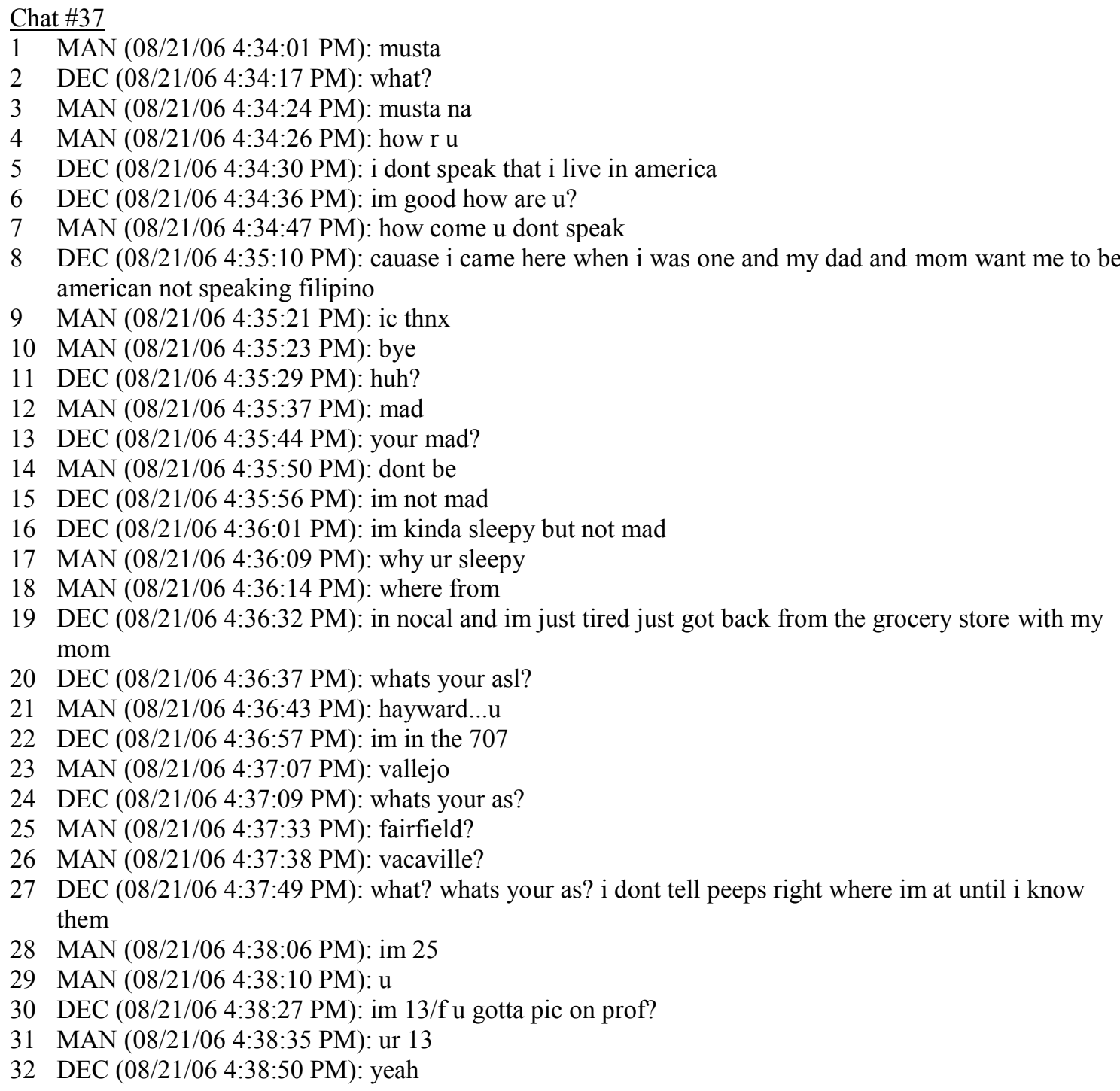


$33 \operatorname{MAN}(08 / 21 / 064: 39: 12 \mathrm{PM})$ : is that $u$ on pic

34 DEC (08/21/06 4:39:25 PM): yeah and my pics on myspace and a couple more on yahoo photo

35 MAN (08/21/06 4:39:35 PM): ur pretty

36 DEC (08/21/06 4:39:40 PM): thank u very much :)

37 MAN (08/21/06 4:39:49 PM): im old

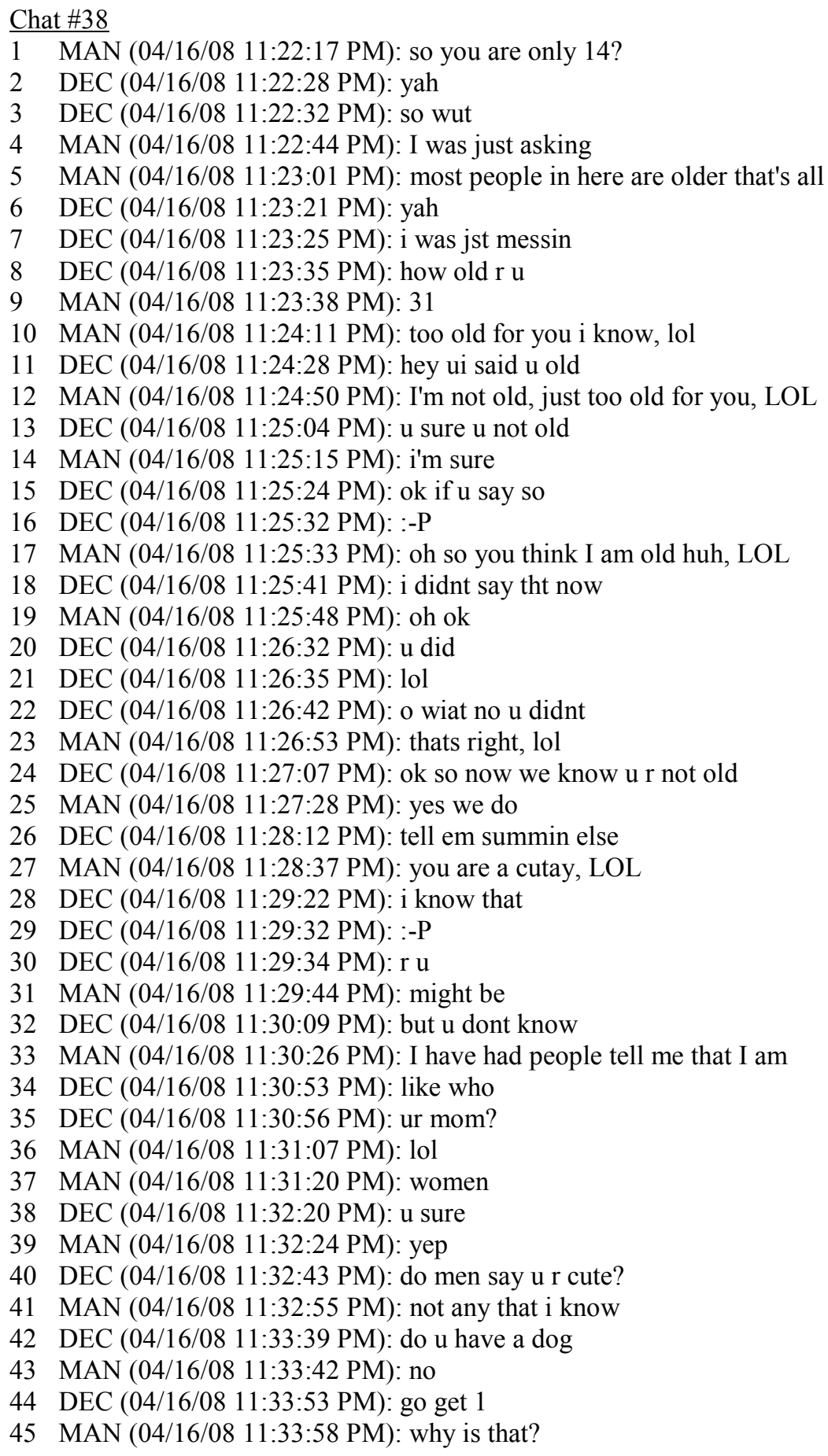

Chat \#39 
1 MAN (07/22/06 11:47:58 AM): Hi, I noticed your and wanted to get to know you. How are you? Pic on profile and im box.

2 DEC (07/22/06 11:48:16 AM): u a bot?

3 MAN (07/22/06 11:48:19 AM): No.

4 DEC (07/22/06 11:48:53 AM): cool asl?

5 MAN (07/22/06 11:49:13 AM): 30, male, doraville

6 DEC (07/22/06 11:49:30 AM): $13 \mathrm{fga}$

7 MAN (07/22/06 11:49:35 AM): where in ga?

8 DEC (07/22/06 11:49:46 AM): no of columbus

9 MAN (07/22/06 11:49:53 AM): cool

10 MAN (07/22/06 11:50:10 AM): I bet I am too old for you and like it says I am bored and naked.

11 DEC (07/22/06 11:50:32 AM): lol u not old

12 MAN (07/22/06 11:50:48 AM): Do you have more pictures?

13 DEC (07/22/06 11:51:02 AM): u gotta pic?

14 MAN (07/22/06 11:51:14 AM): on profile....

15 MAN (07/22/06 11:51:17 AM): and cam

16 DEC (07/22/06 11:51:47 AM): k lemme check

17 DEC (07/22/06 11:52:12 AM): wow ur cute!

18 MAN (07/22/06 11:52:25 AM): thanks

19 DEC (07/22/06 11:52:47 AM): so wats ur name?

20 MAN (07/22/06 11:52:54 AM): Jeff

21 DEC (07/22/06 11:53:14 AM): hi jeff im maddie

22 MAN (07/22/06 11:53:19 AM): nice to meet you

23 DEC (07/22/06 11:53:34 AM): u too

24 MAN (07/22/06 11:54:00 AM): so what interests you about me?

25 DEC (07/22/06 11:54:58 AM): ur eyes i gess $n$ i like italians

26 MAN (07/22/06 11:55:22 AM): thanks

27 MAN (07/22/06 11:55:29 AM): do you have more pictures?

28 DEC (07/22/06 11:55:44 AM): yeah

29 MAN (07/22/06 11:55:49 AM): may I see?

30 DEC (07/22/06 11:55:57 AM): lol u got more?

31 MAN (07/22/06 11:56:13 AM): a cam

32 DEC (07/22/06 11:56:36 AM): cool

33 DEC (07/22/06 11:56:51 AM): u wanna add me?

34 DEC (07/22/06 11:57:34 AM): ty

35 MAN (07/22/06 11:57:41 AM): you are welcome

36 DEC (07/22/06 11:57:52 AM): i can send pics..email?

37 MAN (07/22/06 11:58:06 AM): @yahoo.com

38 DEC (07/22/06 11:58:18 AM): k gimme a sec k?

$39 \operatorname{MAN}(07 / 22 / 06$ 11:58:21 AM): ok

\section{Chat \#40}

1 MAN (5:57:38 PM): hi

2 DEC (3/24/2006 3:57:23 PM): heya!

3 MAN (3/24/2006 3:57:42 PM): so9 what $r \mathrm{u}$ into tonight

4 DEC (3/24/2006 3:58:14 PM): not much its boring

5 MAN (3/24/2006 3:58:20 PM): $r$ u there

6 DEC (3/24/2006 3:58:27 PM): yah im here

7 MAN (3/24/2006 3:58:33 PM): same here

8 DEC (3/24/2006 3:58:40 PM): whats your asl?

9 MAN (3/24/2006 3:59:04 PM): do u really want to know

10 DEC (3/24/2006 3:59:25 PM): thats why i asked lol

11 MAN (3/24/2006 3:59:52 PM): iam m 40 greenfeild ohio and $u$

12 DEC (3/24/2006 4:00:25 PM): im 15/f and im in greenville!

$13 \operatorname{MAN}(3 / 24 / 2006$ 4:00:47 PM): i guess iam to old 
$14 \operatorname{DEC}(3 / 24 / 2006$ 4:00:54 PM): lol i dunno are you cool?

$15 \operatorname{MAN}(3 / 24 / 2006$ 4:01:02 PM): u bet

16 MAN (3/24/2006 4:01:10 PM): y

17 DEC (3/24/2006 4:01:28 PM): i dunno as long as your cool

$18 \operatorname{MAN}(3 / 24 / 2006$ 4:01:40 PM): iam bored

$19 \operatorname{DEC}(3 / 24 / 2006$ 4:01:45 PM): yah me too

20 DEC (3/24/2006 4:01:49 PM): i hate small town living lol

21 MAN (3/24/2006 4:02:06 PM): me to where greenville

$22 \operatorname{DEC}(3 / 24 / 2006$ 4:02:16 PM): outskirts of it really

$23 \operatorname{MAN}(3 / 24 / 2006$ 4:02:34 PM): where

24 DEC (3/24/2006 4:02:40 PM): huh?

25 MAN (3/24/2006 4:02:52 PM): iam 1hr south of columbus

26 MAN (3/24/2006 4:03:34 PM): what the biggest town close to u

27 DEC (3/24/2006 4:03:44 PM): im like thirty miles northwest of dayton

28 MAN (3/24/2006 4:04:02 PM): not far from here

29 DEC (3/24/2006 4:04:11 PM): yah its real close

30 MAN (3/24/2006 4:04:39 PM): so iam drinking what $r u$ doing

31 DEC (3/24/2006 4:04:53 PM): im just sitting bored

32 DEC (3/24/2006 4:05:00 PM): friday night in the boonies lol

33 MAN (3/24/2006 4:05:13 PM): on a friday night

34 DEC (3/24/2006 4:05:30 PM): yah it sucks

35 MAN (3/24/2006 4:05:50 PM): well to bad u cant drink

36 DEC (3/24/2006 4:06:01 PM): lol yah i never drink nothing

37 MAN (3/24/2006 4:06:12 PM): see good

38 DEC (3/24/2006 4:06:22 PM): i was being joking

$39 \operatorname{MAN}(3 / 24 / 2006$ 4:06:30 PM): oh

Chat \#41

1 MAN (05/24/08 10:04:53 AM): hello how are you ?

2 DEC (05/24/08 10:05:30 AM): good

3 DEC (05/24/08 10:05:31 AM): u?

$4 \operatorname{MAN}(05 / 24 / 08$ 10:06:12 AM): 26/m/ut

5 MAN (05/24/08 10:06:21 AM): u ?

6 DEC (05/24/08 10:06:37 AM): $13 \mathrm{fut}$

7 DEC (05/24/08 10:06:40 AM): im ruby

8 DEC (05/24/08 10:06:43 AM): whats ur name?

9 MAN (05/24/08 10:06:55 AM): Felipe here nice to meet you

10 MAN (05/24/08 10:07:27 AM): hope u dont mind the age diff Ruby?

11 DEC (05/24/08 10:07:27 AM): nice 2 me u 2

12 DEC (05/24/08 10:07:47 AM): just numbers

13 MAN (05/24/08 10:08:12 AM): yeah hats true but there are some that think diff

14 DEC (05/24/08 10:08:25 AM): who?

15 MAN (05/24/08 10:08:29 AM): so your in ut? ogden here

16 MAN (05/24/08 10:08:43 AM): lots of othere people

17 MAN (05/24/08 10:09:42 AM): so u ready for the weekend ??

18 DEC (05/24/08 10:10:20 AM): ya

19 DEC (05/24/08 10:10:27 AM): schools out

20 DEC (05/24/08 10:10:28 AM): yay

21 MAN (05/24/08 10:10:38 AM): lol yeah true

22 MAN (05/24/08 10:10:50 AM): u doing anything special ?

23 DEC (05/24/08 10:11:00 AM): no

$24 \operatorname{MAN}(05 / 24 / 08$ 10:11:20 AM): no lol why?

25 DEC (05/24/08 10:11:56 AM): cause nothin 2 do

26 DEC (05/24/08 10:11:58 AM): brb

$27 \operatorname{MAN}(05 / 24 / 08$ 10:12:15 AM): ok 
28 DEC (05/24/08 10:25:23 AM): sorry bout that

29 DEC (05/24/08 10:25:24 AM): back

$30 \operatorname{MAN}(05 / 24 / 08$ 10:26:23 AM): thats ok lol

31 DEC (05/24/08 10:26:29 AM): dog wanted out

32 MAN (05/24/08 10:26:49 AM): ic cool what kind?

33 DEC (05/24/08 10:27:49 AM): dashound

34 DEC (05/24/08 10:27:56 AM): dont know how 2 spell it lol

35 MAN (05/24/08 10:27:59 AM): ic cool

36 DEC (05/24/08 10:28:00 AM): wennie dog

37 DEC (05/24/08 10:28:06 AM): shes my gmas dog

38 MAN (05/24/08 10:28:20 AM): cool

39 MAN (05/24/08 10:29:23 AM): so what part of ut $r u$ in ?

40 DEC (05/24/08 10:30:30 AM): ***EDIT***

41 MAN (05/24/08 10:31:26 AM): wow ogden here

$42 \operatorname{MAN}(05 / 24 / 08$ 10:31:35 AM): we are far

Chat \#42

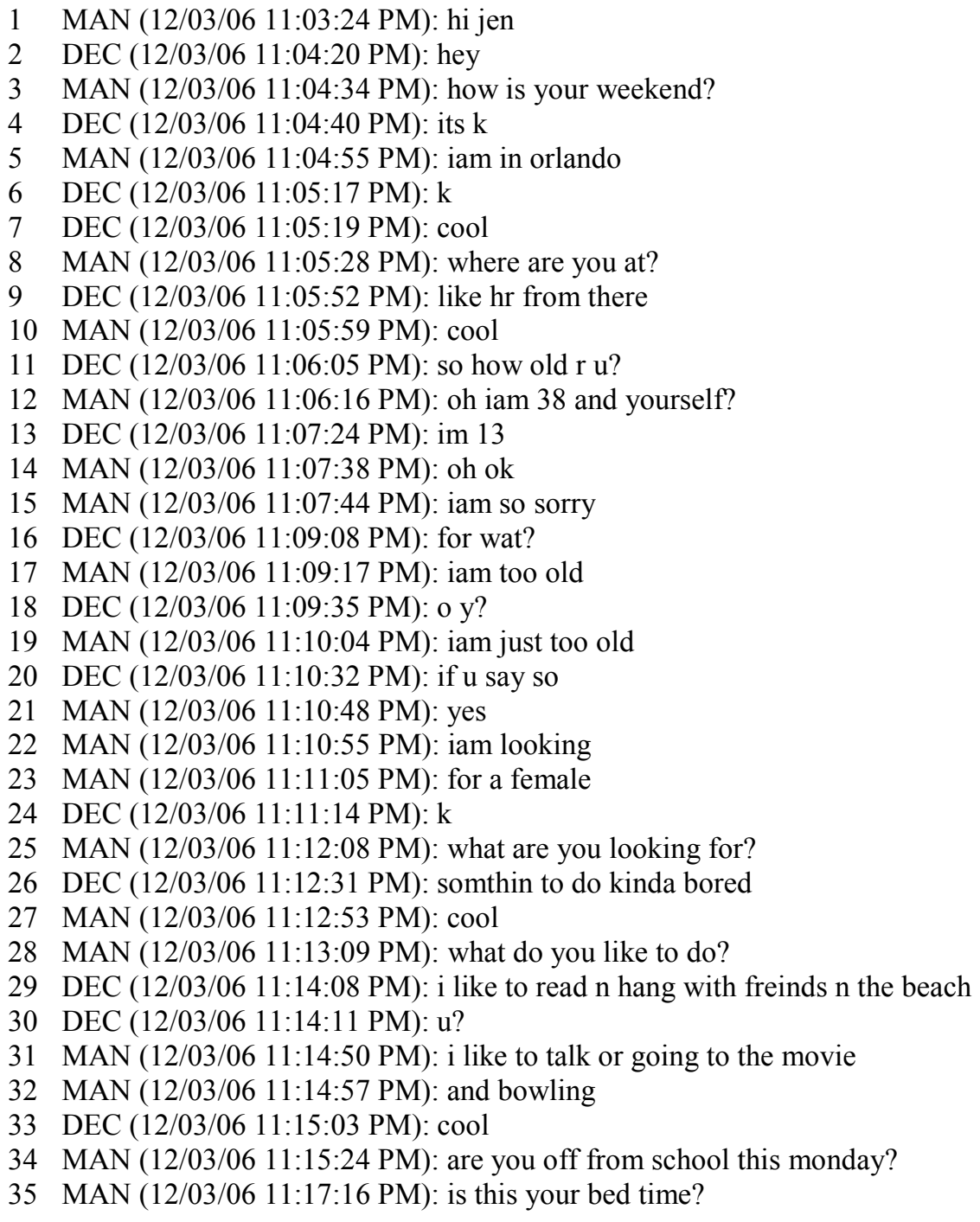

Chat \#43

1 MAN (5:42:30 PM): hey 


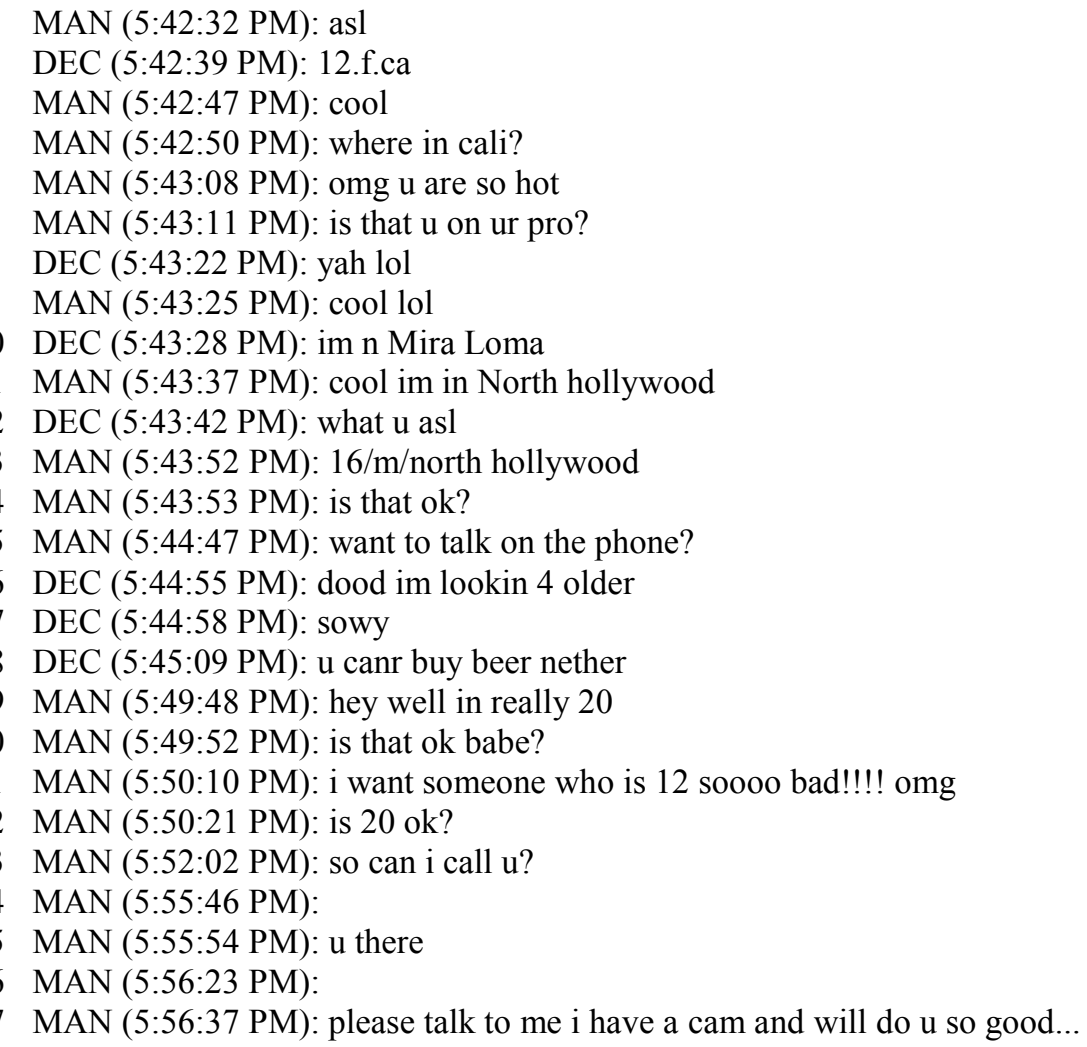

\section{Chat \#44}

1 MAN (09/04/06 10:11:31 PM): hello there

2 DEC (09/04/06 10:11:38 PM): hey

3 DEC (09/04/06 10:11:39 PM): asl

4 MAN (09/04/06 10:11:51 PM): M 29 and $u$

5 DEC (09/04/06 10:12:01 PM): 13/f/ca

6 DEC (09/04/06 10:12:04 PM): $r$ u in ca?

7 MAN (09/04/06 10:12:20 PM): yes

8 DEC (09/04/06 10:12:24 PM): where at?

9 MAN (09/04/06 10:12:36 PM): LA and u

10 DEC (09/04/06 10:12:47 PM): i'm visiting my aunt in la

11 DEC (09/04/06 10:12:48 PM): well

12 DEC (09/04/06 10:12:49 PM): close

13 MAN (09/04/06 10:13:01 PM): thats cool

14 MAN (09/04/06 10:13:03 PM): how old are u

15 DEC (09/04/06 10:13:39 PM): i said already silly

16 DEC (09/04/06 10:13:46 PM): look up ^

17 MAN (09/04/06 10:13:50 PM): lol

18 MAN (09/04/06 10:13:56 PM): what nationaltiy are $u$

$19 \operatorname{DEC}(09 / 04 / 06$ 10:14:25 PM): i'm sorta a mutt

20 DEC (09/04/06 10:14:34 PM): all sorts of stuff

21 DEC (09/04/06 10:14:34 PM): lol

22 DEC (09/04/06 10:14:37 PM): what $\mathrm{r} u$ ?

23 DEC (09/04/06 10:15:30 PM): hello?

$24 \operatorname{MAN}(09 / 04 / 06$ 10:16:01 PM): what nationality are $\mathrm{u}$

25 DEC (09/04/06 10:16:06 PM): i said i'm all kinds

26 DEC (09/04/06 10:16:10 PM): what $\mathrm{r} u$ ?

27 MAN (09/04/06 10:16:20 PM): are u white black hispanic? 
28 DEC (09/04/06 10:16:21 PM): i'm asian, i'm portuguese, i'm dutch

29 MAN (09/04/06 10:16:28 PM): oh interesting

30 MAN (09/04/06 10:16:37 PM): i wonder what that looks like

31 MAN (09/04/06 10:16:56 PM): im hispanic

32 DEC (09/04/06 10:17:06 PM): i have a pic in my profile

33 DEC (09/04/06 10:17:11 PM): do u have a pic/

34 MAN (09/04/06 10:17:16 PM): let me see

35 MAN (09/04/06 10:18:35 PM): you know ur very young and im a lot older than $\mathrm{u}$

36 MAN (09/04/06 10:18:43 PM): how old is ur aunt

37 DEC (09/04/06 10:18:57 PM): she's like in her 40's why?

38 MAN (09/04/06 10:19:04 PM): oh just asking

$39 \operatorname{DEC}(09 / 04 / 06$ 10:19:04 PM): and age is soooo just a number

40 MAN (09/04/06 10:19:14 PM): not when ur 13

41 MAN (09/04/06 10:19:20 PM): Im 29

42 DEC (09/04/06 10:19:41 PM): yeah, it is

43 DEC (09/04/06 10:19:47 PM): cos if u're nice u're nice

44 DEC (09/04/06 10:19:54 PM): it doesn't matter if u're 12 or 112

$45 \operatorname{MAN}(09 / 04 / 06$ 10:20:00 PM): lol

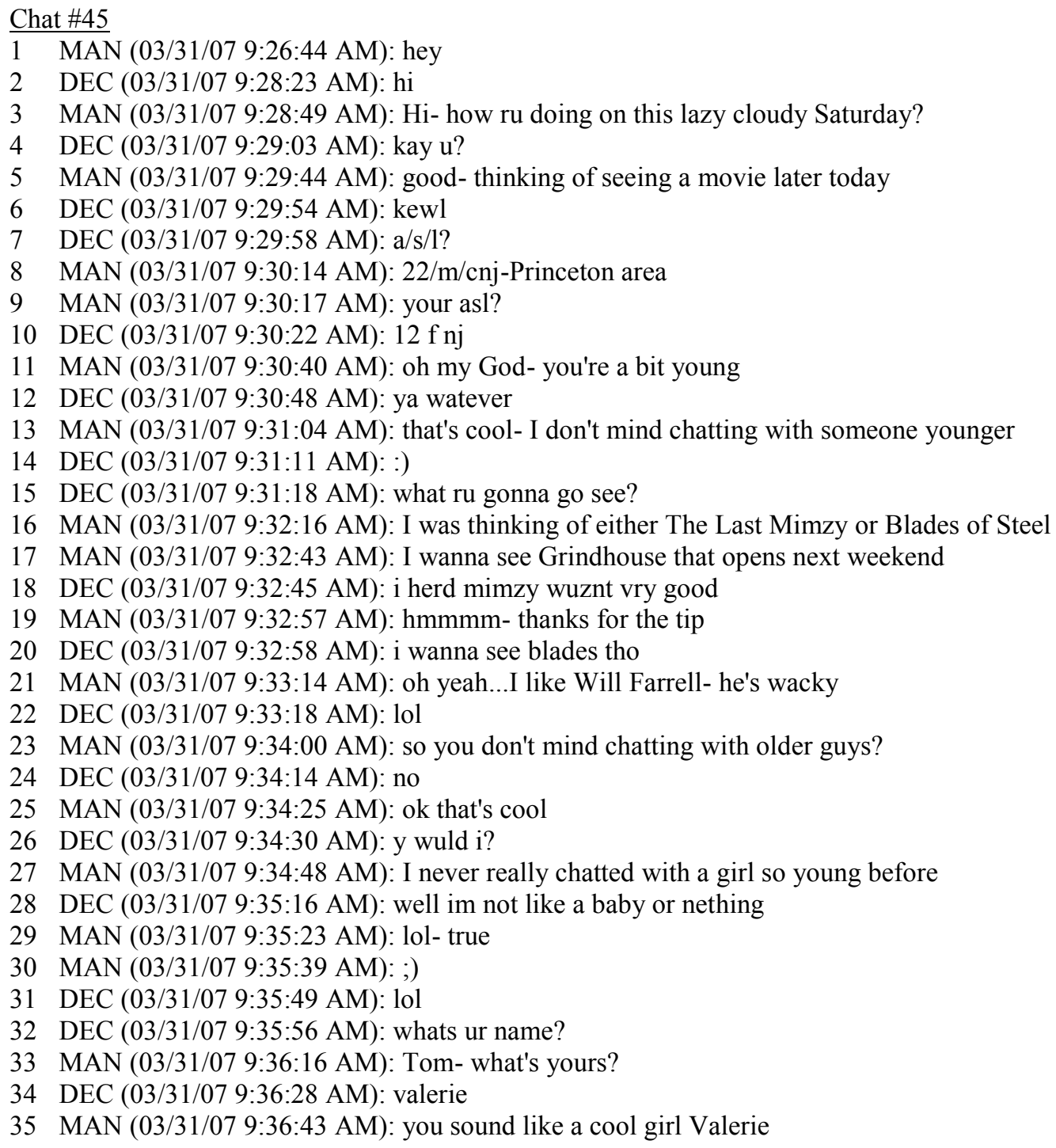


$36 \operatorname{DEC}(03 / 31 / 07$ 9:36:50 AM): ty

37 MAN (03/31/07 9:37:32 AM): so what do you like to do for fun? I like to go snowboarding, watch cool and funny movies, play video games, so silly stuff with frineds

38 DEC (03/31/07 9:38:12 AM): i like to play with my dogs, hang out with my friends, go shopping, talk on here, go 2 movies

$39 \operatorname{MAN}(03 / 31 / 07$ 9:38:22 AM): cool

\section{Chat \#46}

1 MAN (09/17/07 9:22:46 PM): hi

2 MAN (09/17/07 9:22:53 PM): asl plz?

3 DEC (09/17/07 9:23:00 PM): hi

4 DEC (09/17/07 9:23:13 PM): 13/f/ky

5 MAN (09/17/07 9:23:26 PM): wow, you're just a young one huh/

6 MAN (09/17/07 9:23:30 PM): @\};-

7 MAN (09/17/07 9:23:40 PM): a little cutie too

8 MAN (09/17/07 9:24:39 PM): if nobody will talk to you sweetheart you can talk to me........i'm a good guy, i have 2 nieces your age

9 DEC (09/17/07 9:24:59 PM): really

10 DEC (09/17/07 9:25:08 PM): wats ur asl

11 MAN (09/17/07 9:25:45 PM): well, i should say i had 2 nieces your age, when i lived in washington state they were your age, they're a little older now but it still feels to me like they're your age

12 MAN (09/17/07 9:25:58 PM): i'm 36/m/nashville

13 DEC (09/17/07 9:26:08 PM): o

14 DEC (09/17/07 9:26:12 PM): nashville kewl

15 MAN (09/17/07 9:26:27 PM): you must be bored if you're on the internet

16 MAN (09/17/07 9:26:40 PM): unless you think it's fun lol

17 MAN (09/17/07 9:27:21 PM): that's me

18 MAN (09/17/07 9:27:39 PM): those are my 2 dogs

19 DEC (09/17/07 9:28:01 PM): kewl

20 MAN (09/17/07 9:28:08 PM): the black one is Bud and the little white one is Daisy

21 DEC (09/17/07 9:28:18 PM): aww there cute

22 MAN (09/17/07 9:28:20 PM): i have a cam too if you'd like to see me on cam

23 MAN (09/17/07 9:28:49 PM): you can see Bud on cam too, i gave daisy away to my nephew before i moved here to nashville

24 DEC (09/17/07 9:29:09 PM): aww u gave her away

25 MAN (09/17/07 9:29:50 PM): yeah, i really wasn't very happy too but $i$ thought it would be best for her because my nephew and his wife have a dog just like her

26 DEC (09/17/07 9:31:37 PM): kewl

27 MAN (09/17/07 9:31:51 PM): did you get the other 2 pics i sent you?

28 DEC (09/17/07 9:32:15 PM): of ur dog yeah

29 MAN (09/17/07 9:32:20 PM): ok

30 MAN (09/17/07 9:32:30 PM): want to see me on cam?

31 DEC (09/17/07 9:33:55 PM): shur

32 MAN (09/17/07 9:34:32 PM): look at those big blue eyes........you're so cute lol

\section{Chat \#47}

MAN (09/09/06 6:28:26 PM): hi

MAN (09/09/06 6:28:28 PM): asl?

DEC (09/09/06 6:28:42 PM): heya whats up?

DEC (09/09/06 6:28:47 PM): 13/f/socal whats ur asl?

MAN (09/09/06 6:28:52 PM): k

MAN (09/09/06 6:28:55 PM): $\mathrm{m} / 29$ socal here

DEC (09/09/06 6:29:10 PM): cool u gotta pic on prof?

MAN (09/09/06 6:29:22 PM): yeah go check it

MAN (09/09/06 6:29:25 PM): :) 
10 DEC (09/09/06 6:30:06 PM): u cute u look tough

11 MAN (09/09/06 6:30:19 PM): lol thanx

12 DEC (09/09/06 6:30:50 PM): yw

13 DEC (09/09/06 6:30:55 PM): i got my pic on prof and more on yahoo photo

14 MAN (09/09/06 6:31:19 PM): sweet

15 MAN (09/09/06 6:31:22 PM): u look cute too

16 DEC (09/09/06 6:31:29 PM): lol thank u :)

17 MAN (09/09/06 6:32:05 PM): where in cali?

18 DEC (09/09/06 6:32:17 PM): socal

19 MAN (09/09/06 6:32:32 PM): where?

20 MAN (09/09/06 6:32:33 PM): me too

21 MAN (09/09/06 6:32:40 PM): i'm near la

22 MAN (09/09/06 6:32:44 PM): well near the valley

23 DEC (09/09/06 6:32:45 PM): im 40 min from la

$24 \operatorname{MAN}(09 / 09 / 06$ 6:32:47 PM): me too

25 DEC (09/09/06 6:32:49 PM): cool

26 MAN (09/09/06 6:32:50 PM): i'm north

27 MAN (09/09/06 6:32:52 PM): of la

28 DEC (09/09/06 6:32:57 PM): oh okay

$29 \operatorname{MAN}(09 / 09 / 06$ 6:33:00 PM): u?

30 DEC (09/09/06 6:33:06 PM): im in the 526

31 MAN (09/09/06 6:33:13 PM): dunno where that is

32 MAN (09/09/06 6:33:15 PM): it's cool

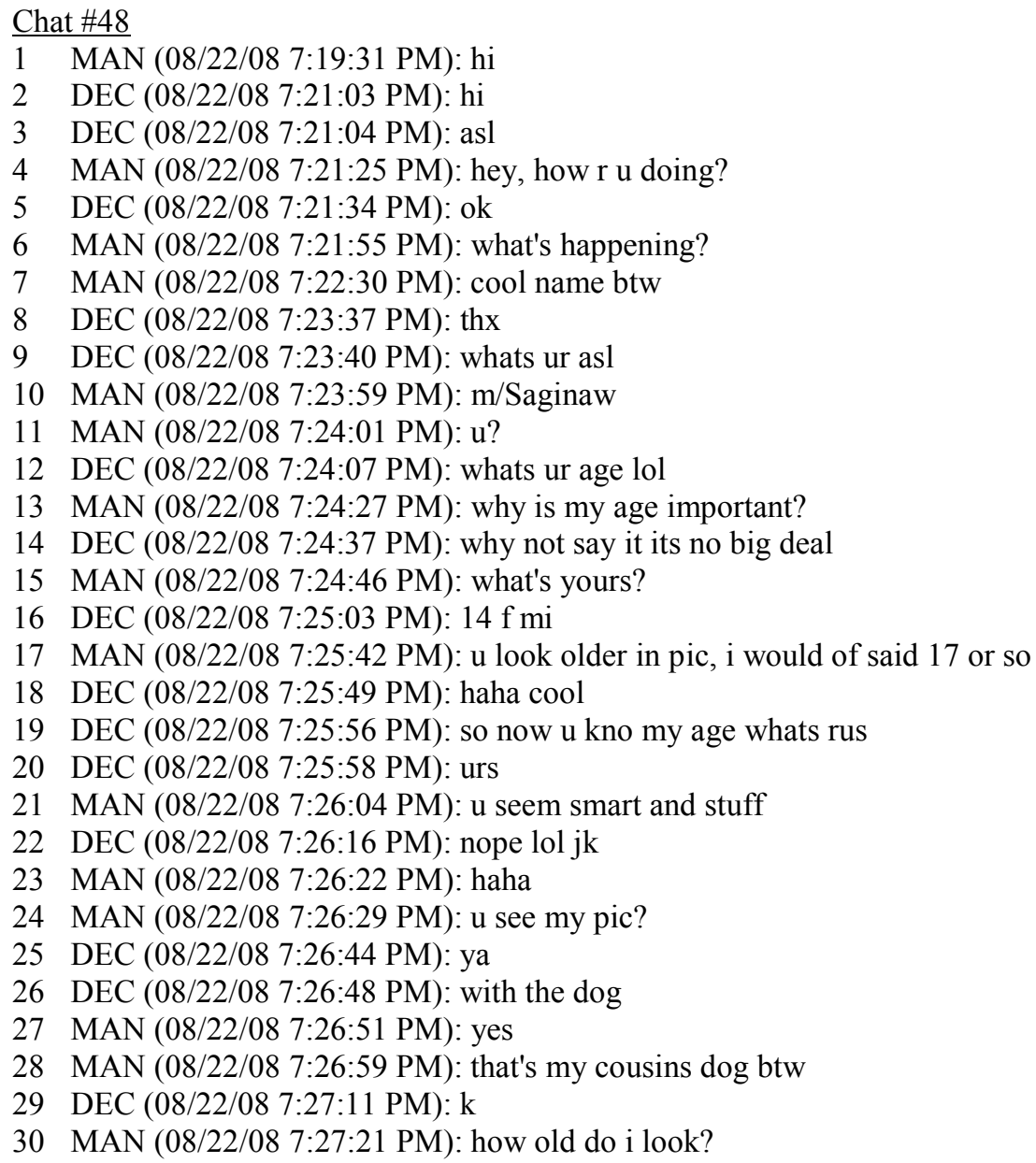


31 DEC (08/22/08 7:27:34 PM): idk but u act 10

32 MAN (08/22/08 7:27:39 PM): awww

33 MAN (08/22/08 7:27:44 PM): what?

34 MAN (08/22/08 7:27:48 PM): why do u say that?

35 DEC (08/22/08 7:27:59 PM): bcuz ur a baby

$36 \operatorname{MAN}(08 / 22 / 08$ 7:28:10 PM): well $\mathrm{i}$ am the baby of the family

37 DEC (08/22/08 7:28:43 PM): $u$ act like it

38 MAN (08/22/08 7:28:47 PM): aww

Chat \#49

1 first line was: do $\mathrm{u}$ know who anna leda is?

2 DEC (9:20:22 PM): no

3 DEC (9:20:33 PM): who is she

4 MAN (9:20:52 PM): is my younger sis, she still lives out there in the falls

5 DEC (9:21:41 PM): kewl

6 DEC (9:21:54 PM): asl

7 MAN (9:22:54 PM): 24/m/waukesha,... relax not hittin on ya, ur from the falls, same age as my sis, was just wondering if ya knew her

8 DEC (9:23:10 PM): $\mathrm{k}$

9 MAN (9:24:18 PM): its been years since i been out there in the falls

10 DEC (9:24:59 PM): i like it

11 DEC (9:25:02 PM): its ok

12 MAN (9:25:34 PM): its a party town, always had some wild ones out there

13 DEC (9:25:39 PM): hehe

14 DEC (9:26:02 PM): i am 14 i cant go 2 them kinds of partys yet

15 DEC (9:26:10 PM): mom wont let me

$16 \operatorname{MAN}(9: 26: 38 \mathrm{PM})$ : when i was 14 , i was expelled for the sec time from junior high

17 DEC (9:26:47 PM): wow hehe y?

18 MAN (9:27:11 PM): caught with pot on school grounds

19 DEC (9:27:18 PM): that sux

20 MAN (9:27:25 PM): yeppers

21 DEC (9:27:33 PM): i bet they kept it

22 MAN (9:27:49 PM): the part they found they kept

23 MAN (9:28:00 PM): i didnt vring all my stash to school

24 MAN (9:28:05 PM): bring

25 DEC (9:28:07 PM): kewl

26 DEC (9:28:39 PM): it smels good

27 MAN (9:29:23 PM): now $i$ thinkin bout it, anna a year older then $u$, u might know jeol, or my cousin rebecca

28 DEC (9:29:53 PM): no sry

29 DEC (9:30:00 PM): i am kinda new her

30 DEC (9:30:02 PM): e

31 MAN (9:30:31 PM): ahhh ok,.. i was gonna say, its still a small town, everyone knows everybody

32 DEC (9:30:46 PM): i moved here with my mom

33 DEC (9:30:54 PM): we lived in ky

34 DEC (9:31:16 PM): i dont see my dad much

35 MAN (9:31:19 PM): i been there,.. is very diff

36 DEC (9:31:25 PM): yea it is

37 DEC (9:31:33 PM): i miss my friends in ky

38 MAN (9:31:52 PM): i dont see either of my folks,

39 DEC (9:32:04 PM): y not

40 DEC (9:32:12 PM): u live far from them

41 MAN (9:32:34 PM): there dead beats, pops i think may be in prison, and my mas is just crazy

42 DEC (9:32:54 PM): wow that sux

43 DEC (9:33:06 PM): $\mathrm{y}$ is he in prison 
44 MAN (9:33:22 PM): i am over it, its been almost 10 yrs since i talked to em

45 DEC (9:33:29 PM): thats kewl

46 MAN (9:33:32 PM): drugs and younger girls

47 MAN (9:33:49 PM): y my pops in prison

48 DEC (9:33:58 PM): wow

49 DEC (9:34:05 PM): how long

50 MAN (9:34:59 PM): dont know, rumor has it like 15 yrs... but my older sis in texas told me, and she a crack head and talks a lot of shit, so i dont know if any of it is true

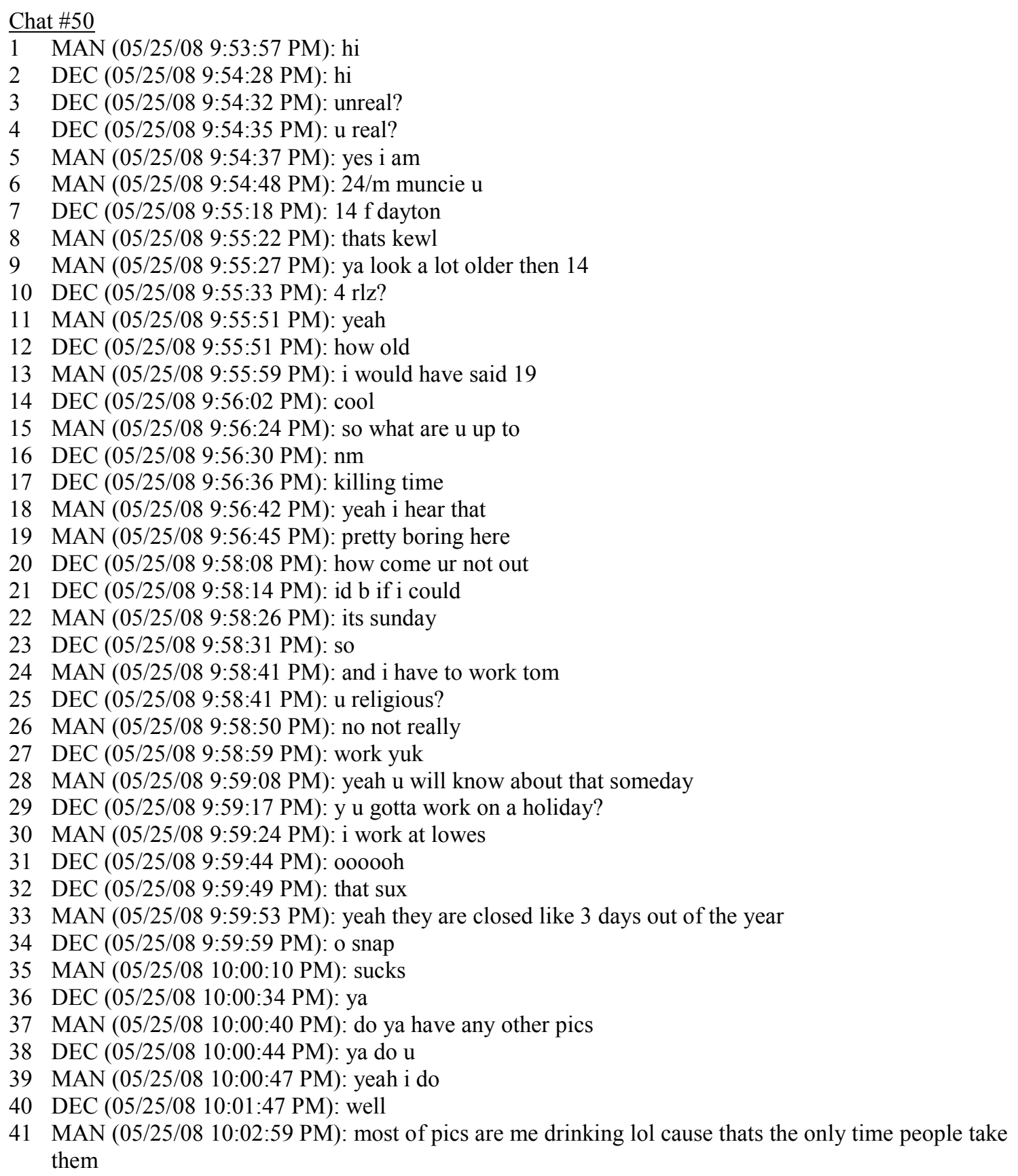

Chat \#51

1 MAN (4/13/2006 11:20:00 PM): hiya liz or beth 


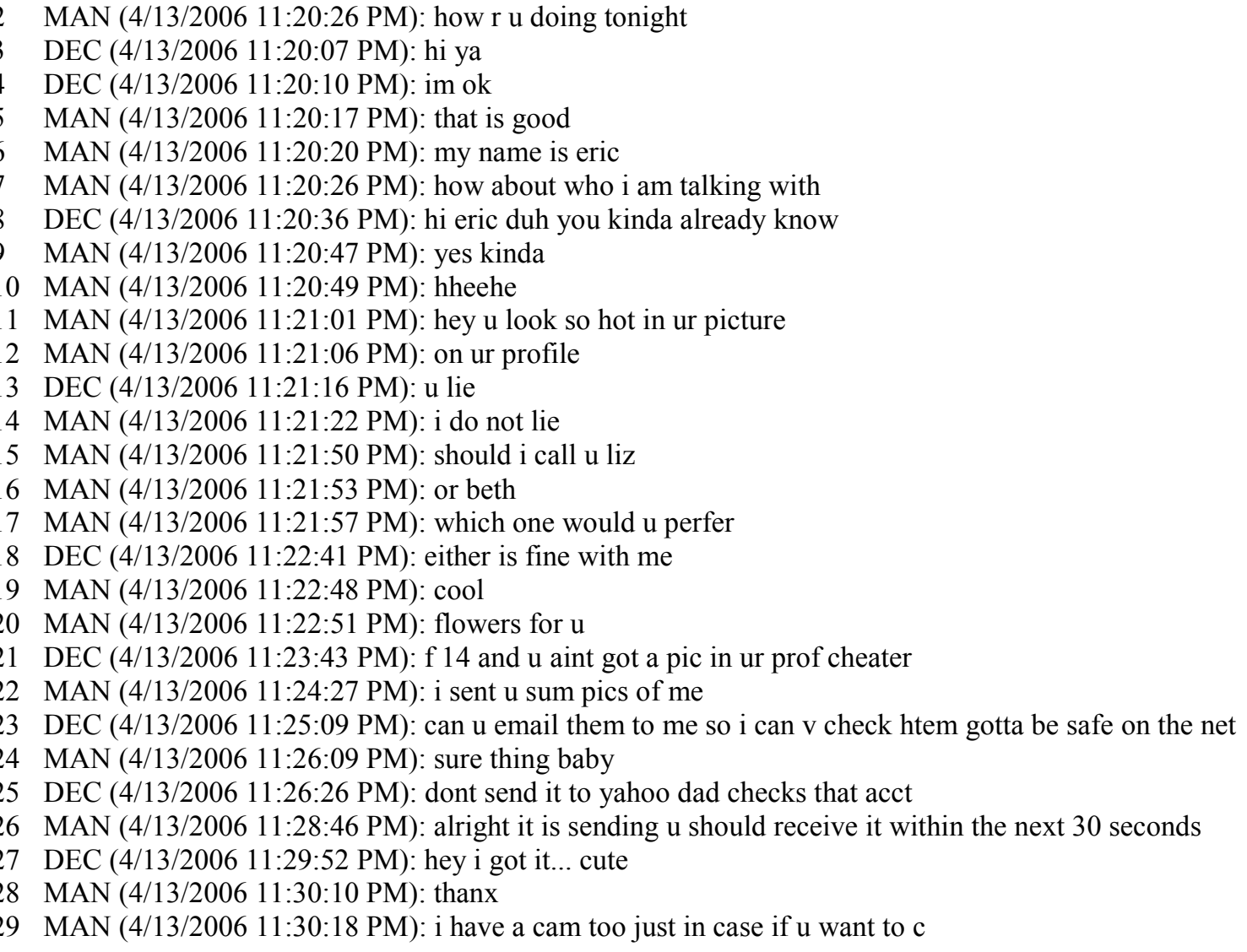

\section{Chat \#52}

1 MAN (03/27/08 10:54:26 PM): hi

2 MAN (03/27/08 10:54:32 PM): 28/male/nor cal

3 MAN (03/27/08 10:54:37 PM): how is it going?

4 DEC (03/27/08 10:55:27 PM): hello 13/f/ca

5 MAN (03/27/08 10:55:46 PM): cool

6 MAN (03/27/08 10:55:53 PM): what $\mathrm{r}$ u up to?

7 DEC (03/27/08 10:56:04 PM): nm, supposed to be working on homework

8 MAN (03/27/08 10:56:33 PM): hahhahahaha

9 MAN (03/27/08 10:56:34 PM): cool

10 DEC (03/27/08 10:56:53 PM): lol

11 MAN (03/27/08 10:56:59 PM): am sort of bored here

12 MAN (03/27/08 10:57:05 PM): got back frm work now

13 MAN (03/27/08 10:57:10 PM): kinda relaxing

$14 \operatorname{MAN}(03 / 27 / 08$ 10:57:14 PM): and killing time

15 DEC (03/27/08 10:57:15 PM): what do you do?

16 MAN (03/27/08 10:57:17 PM): lol

17 MAN (03/27/08 10:57:31 PM): i work as software engineer

18 DEC (03/27/08 10:57:39 PM): whats that?

19 MAN (03/27/08 10:57:53 PM): computer programmer

20 DEC (03/27/08 10:58:06 PM): wow, u r smart huh

21 MAN (03/27/08 10:58:18 PM): yes

22 MAN (03/27/08 10:58:30 PM): u wanna make friendship with smart guy..huh?

23 DEC (03/27/08 10:58:46 PM): yeah

24 MAN (03/27/08 10:58:52 PM): cool

25 MAN (03/27/08 10:59:01 PM): i'll add u in my buddy list then 
26 DEC (03/27/08 10:59:13 PM): kewl can i add you too?

27 MAN (03/27/08 10:59:17 PM): sure

28 MAN (03/27/08 10:59:19 PM): :)

\section{Chat \#53}

1 MAN (1:59:52 PM): hi

2 MAN (1:59:59 PM): u want too see my cam

3 DEC (2:00:47 PM): uhh

4 DEC (2:00:51 PM): well not really lol

5 DEC (2:00:54 PM): asl?

6 MAN (2:00:59 PM): mmmmmmmmm lol y not

7 MAN (2:01:03 PM): 24

8 MAN (2:01:13 PM): can i excite u

9 DEC (2:01:13 PM): cuz i don't even know you! lol

10 DEC (2:01:24 PM): im 13 f riverside where are you from

11 MAN (2:01:26 PM): not yet

12 MAN (2:01:36 PM): hollywood

13 MAN (2:01:44 PM): $\mathrm{u}$ have a pic

14 DEC (2:02:00 PM): no do u

15 MAN (2:02:04 PM): $\mathrm{mmmmmmm}$ your young

16 MAN (2:02:12 PM): no a cam

17 MAN (2:02:17 PM): are u horny

18 DEC (2:02:23 PM): lol i dunno..

19 MAN (2:02:38 PM): u r arnt u

20 DEC (2:02:45 PM): well not yet!

21 MAN (2:02:48 PM): i am

22 MAN (2:02:53 PM): mmmmmmmmmmmmmmmmmmmmmmmmmmmmmm ok

23 MAN (2:03:01 PM): mayb e we can change that

24 MAN (2:03:07 PM): what $r$ u wearing

25 MAN (2:03:11 PM): this morning

26 MAN (2:03:15 PM): are $u$ hot looking

27 DEC (2:03:16 PM): camo shorts and a tank

28 MAN (2:03:25 PM): mmmmmm

29 DEC (2:03:28 PM): im 5'2 longish brown hair green eyes

30 DEC (2:03:29 PM): you?

31 MAN (2:03:33 PM): $r$ u a virgin

\section{Chat \#54}

1 MAN: You dont look that young

2 DEC: $y$ ?

3 MAN: I was bouncing through profiles and found yours.. was surprised between your age and your pic.

4 DEC: how come

5 MAN: you just look older

6 DEC: must be the makeup

7 DEC: 101

8 MAN: lol.. or the big fist in the pic

9 DEC: i was feelin punchy

10 MAN: I see. Do you have a cam?

11 DEC: not anymore

12 MAN: lost it?

13 MAN: parents found out you were showing more than you should on it?

14 DEC: nope mom took it lol

15 MAN: lol

16 DEC: what can i say 
17 MAN: did she walk in on you?

18 DEC: nope i just bought it with my own $\$$ and didn't tell her and she fliped when she saw it

19 MAN: ahhh.. I see.

20 MAN: well, that is good she is watcing out for you.. lots of pervs out there

21 DEC: yeah

22 MAN: what part of WI are you in?

23 DEC: the falls

24 MAN: Sheboygan Falls?

25 DEC: menomonee fals

26 MAN: oh, that is a nice area. Good schools.

27 DEC: yeah its ok

28 DEC: not alot 2 do sometiems

29 MAN: that is with anywhere... where ever a person lives, tends to be boring for them... they are just used to everything.

30 DEC: yeah i stay busy

31 MAN: what do you like to do?

32 DEC: just chill go online movies mall boys ya know

33 MAN: the normal.

34 DEC: yep lol guess im boring

35 MAN: no.. sounds like you are very normal. Not at all boring.

36 DEC: thankx

37 MAN: are you into any sports?

38 DEC: not really

39 MAN: music? drama?

\section{Chat \#55}

1 MAN: i will chat with you

2 DEC: hi! asl

3 MAN: 26 male pdx

4 MAN: you?

5 DEC: $14 \mathrm{f}$ sherwood

6 DEC: $\mathrm{u}$ know where that is?

7 MAN: hell ya

8 DEC: cool

9 DEC: so whatre u up 2

10 MAN: jus working

11 DEC: lol that sounds borin what do u do

12 MAN: i am actually on the coast right now

13 MAN: real estate appraisal

14 DEC: o i love the coast!!!

15 DEC: what part $\mathrm{r} u$ at

16 MAN: astoria

17 DEC: oo way north lol

18 DEC: but u live $\mathrm{n}$ pdx?

19 MAN: well i go back and forth

20 MAN: so you got a pic?

21 DEC: no do u

22 MAN: yes in my profile

23 DEC: $k$ lemme check

24 DEC: srry its loading.. ive got dail up lol

25 MAN: its all good

26 DEC: oo ur cute!

27 MAN: awe thank you!!!

28 DEC: u gotta dimple?

29 MAN: got two of them 
30 DEC: ooo i like!

31 MAN: you do huh

32 DEC: i want dimples lol

33 MAN: you need to get a pic

34 DEC: i know but my mom wont let me gget a cam

35 MAN: oh

36 MAN: well what do you look like

37 DEC: im 5'4 curly blonde hair green eyes

38 DEC: $\mathrm{n}$ dont make fun of me but im $\mathrm{pg}$

39 MAN: ok

40 MAN: come on give me some numbers like 34-26-34

\section{Chat \#56}

1 MAN (5:15:11 PM): mother nature is going darn good to you

2 DEC(5:15:35 PM): awww ty

3 MAN (5:15:45 PM): 113 huh

4 MAN (5:16:06 PM): you really are very pretty that much is true

5 DEC(5:16:18 PM): well ty

6 MAN (5:16:23 PM): yw

7 DEC(5:16:28 PM): and no i am not 113

8 DEC(5:16:35 PM): but taht wuld $\mathrm{b}$ funny huh?

9 DEC(5:16:41 PM): asl plz?

10 MAN (5:16:48 PM): that much I figured out

11 MAN (5:17:00 PM): I was joking also

12 MAN (5:17:02 PM): southeast ms

13 MAN (5:17:10 PM): too old for ya I'm sure

14 MAN (5:17:47 PM): dosn't hurt to say hello to a beautiful girl though

15 DEC(5:18:02 PM): well ty

16 DEC(5:18:07 PM): wat were u jokin about?

17 MAN (5:18:16 PM): your age

18 MAN (5:18:17 PM): lol

19 DEC(5:18:20 PM): oic lol

20 MAN (5:18:30 PM): what part of Ms ya in

21 MAN (5:18:33 PM): north or south

$22 \operatorname{DEC}(5: 18: 48 \mathrm{PM})$ : i dont kno directions real good

$23 \operatorname{MAN}(5: 18: 55$ PM): ic

24 DEC(5:18:59 PM): mebbe if $i$ get 2 kno u ill tell $u$

25 MAN (5:19:00 PM): up or down

26 MAN (5:19:08 PM): hey it's cool

27 DEC(5:19:10 PM): but u wont tell me how old u r either

28 MAN (5:19:13 PM): was just making converstation

29 MAN (5:19:17 PM): no worries

30 DEC(5:19:21 PM): i kno its kewl

31 MAN (5:19:29 PM): k

32 DEC(5:21:02 PM): so ur not gonna talk 2 me?

33 MAN (5:21:15 PM): sure I am

Chat \#57

\footnotetext{
MAN (03/12/07 3:18:43 PM): hey

MAN (03/12/07 3:18:46 PM): which one are you

MAN (03/12/07 3:18:48 PM): left or right

DEC (03/12/07 3:19:37 PM): left

DEC (03/12/07 3:19:42 PM): asl

MAN (03/12/07 3:19:55 PM): 17/m/LA

7 MAN (03/12/07 3:19:57 PM): where you at
} 
8 DEC (03/12/07 3:20:02 PM): so cal

9 MAN (03/12/07 3:20:08 PM): cool me too

10 MAN (03/12/07 3:20:29 PM): what are you doing today

11 DEC (03/12/07 3:20:47 PM): chatin

12 DEC (03/12/07 3:20:49 PM): oh

13 DEC (03/12/07 3:20:55 PM): im 13/f/socali

$14 \operatorname{MAN}(03 / 12 / 07$ 3:21:00 PM): cool

15 MAN (03/12/07 3:21:07 PM): do you have a bf

16 DEC (03/12/07 3:21:12 PM): no

17 DEC (03/12/07 3:21:20 PM): i like older guys so i ttyl

$18 \operatorname{MAN}(03 / 12 / 07$ 3:21:28 PM): how much older

19 MAN (03/12/07 3:21:38 PM): im actually 23

20 DEC (03/12/07 3:21:51 PM): dam y u lie 2 me

21 MAN (03/12/07 3:21:58 PM): since you were $13 \mathrm{i}$ was kind of scared to tell you

$22 \operatorname{MAN}(03 / 12 / 07$ 3:22:00 PM): sorry

23 DEC (03/12/07 3:22:05 PM): i hate liers

$24 \operatorname{MAN}(03 / 12 / 07$ 3:22:11 PM): im sorry

25 MAN (03/12/07 3:22:14 PM): please forgive me

26 DEC (03/12/07 3:22:23 PM): u got a pic?

27 MAN (03/12/07 3:22:39 PM): i do but its not of my face

28 DEC (03/12/07 3:22:56 PM): yur proly 60 years old $\mathrm{n}$ al rinkly $\mathrm{n}$ shit

$29 \operatorname{MAN}(03 / 12 / 07$ 3:23:03 PM): oh hell no

30 MAN (03/12/07 3:23:09 PM): ill show you

31 DEC (03/12/07 3:23:21 PM): how i beliv u sinc u lied 2 me already

32 MAN (03/12/07 3:23:35 PM): do you want to see my cock pic

33 DEC (03/12/07 3:24:07 PM): ummmm how i gona no if yur not like 60 with a dic pic

$34 \operatorname{MAN}(03 / 12 / 07$ 3:24:12 PM): i swear

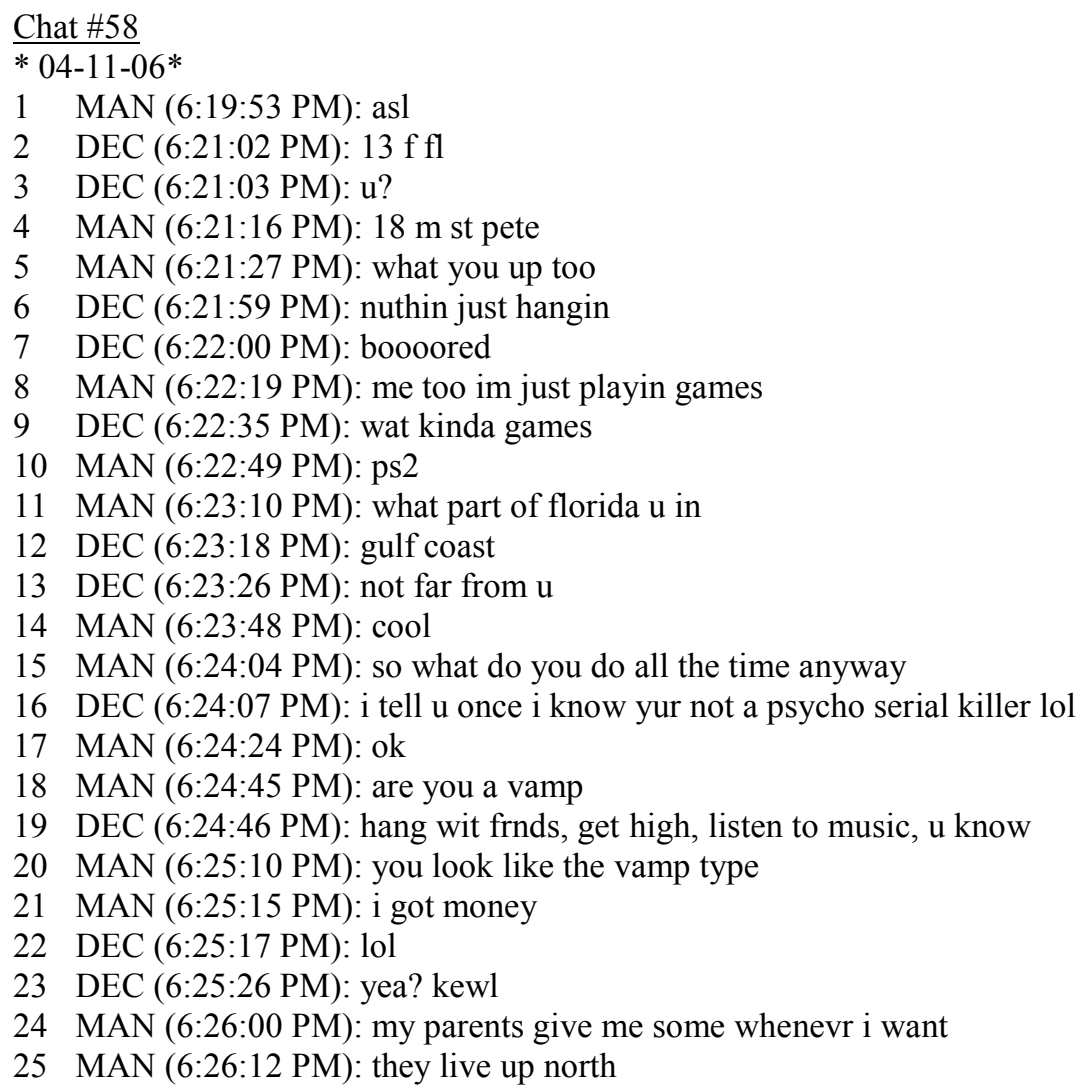


26 DEC (6:26:38 PM): u got yur own place

27 MAN (6:27:00 PM): yeah with everything in it just about

28 DEC (6:27:32 PM): hey i gotta run

29 DEC (6:27:34 PM): mebbe back laterz

30 MAN (6:27:45 PM): add me ok

31 DEC (6:27:49 PM): k

*4-12-06*

32 MAN (5:20:48 PM): hi

33 DEC (5:21:48 PM): hi

34 MAN (5:22:11 PM): what you up too

35 DEC (5:22:33 PM): nuttin just hangin

36 DEC (5:22:51 PM): i made myself invisible coz some stupid kids buggin me

37 MAN (5:23:29 PM): yea i understand but u coulda holla at me

38 DEC (5:23:47 PM): sory i didnt notice u was online

39 MAN (5:24:15 PM): you shold come over my place so we can get high

Chat \#59

1 MAN (9:10:29 PM): hi

2 DEC (9:10:34 PM): hi asl

$3 \operatorname{MAN}(9: 10: 46 \mathrm{PM}): 21 / \mathrm{m} / 714$

4 MAN (9:10:50 PM): u?

5 DEC (9:11:01 PM): 13 f 909

6 MAN (9:11:11 PM): what city?

7 MAN (9:11:24 PM): riverside?

8 DEC (9:11:24 PM): mira loma

9 MAN (9:11:30 PM): hmmm

10 MAN (9:11:41 PM): how far is that from disneyland?

11 DEC (9:11:43 PM): im in riverside co

12 MAN (9:11:51 PM): oh

13 DEC (9:11:55 PM): wow i dunno lol

14 MAN (9:12:00 PM): im in orange county

15 MAN (9:12:09 PM): so are u worth the drive?

16 MAN (9:12:15 PM): :D

17 DEC (9:12:20 PM): how long u gotta drive ? lol

$18 \operatorname{MAN}(9: 12: 32$ PM): like 45 min

19 DEC (9:12:37 PM): thats nuthin

20 DEC (9:12:40 PM): yea im nice

21 MAN (9:12:42 PM): lol

$22 \operatorname{MAN}(9: 12: 48 \mathrm{PM})$ : then $u$ come over here

23 DEC (9:13:00 PM): i wud if i cud drive

24 MAN (9:13:29 PM): so if i was hot and drove way over there, what would we do?

25 DEC (9:13:49 PM): w/e $u$ want, but u gotta be nice. $u$ cant be some killer $r$ sumthin

26 MAN (9:14:02 PM): lol

27 MAN (9:14:08 PM): wanna see my pics?

28 DEC (9:14:33 PM): yeah

29 MAN (9:14:50 PM): edit

30 MAN (9:14:57 PM): tell me what u think

31 DEC (9:15:41 PM): ur hot

32 MAN (9:16:13 PM): how many pics did u get 2 see?

Chat \#60

1 MAN (12:03:40 AM): hi

2 DEC (12:04:14 AM): hi

3 MAN (12:04:47 AM): $21 \mathrm{~m}$ dallas

4 DEC (12:04:50 AM): kool 


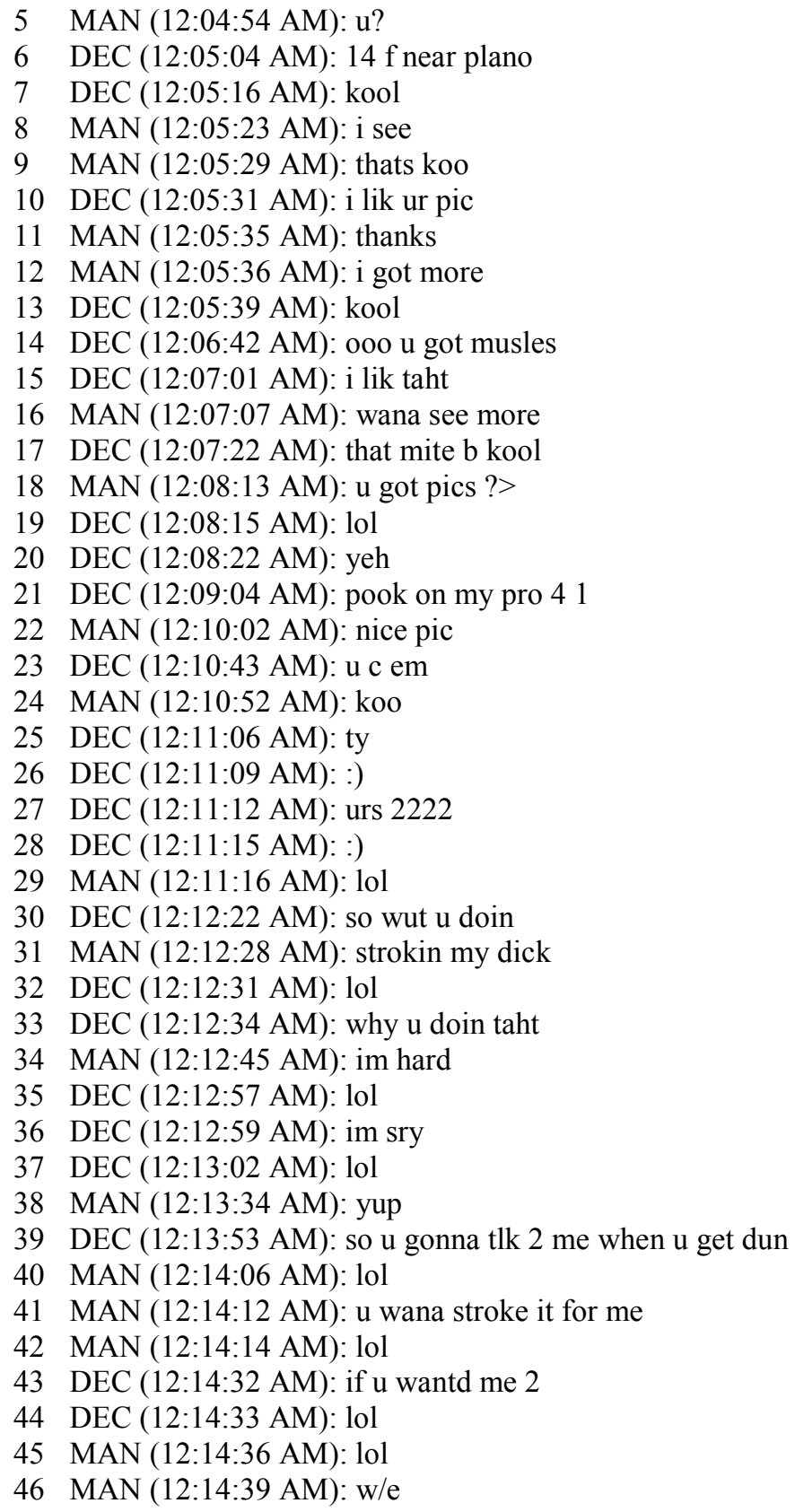

\section{Chat \#61}

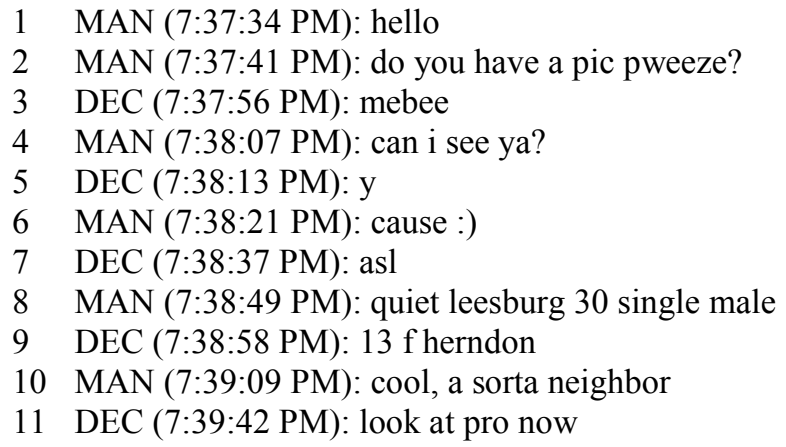


12 MAN (7:40:47 PM): you're pretty!!!

13 MAN (7:40:57 PM): why are you hiding yourself beth?

14 DEC (7:41:04 PM): huh

15 MAN (7:41:19 PM): i mean, why don't you have your pic there always?

$16 \operatorname{DEC}(7: 41: 37 \mathrm{PM})$ : too many pms

17 MAN (7:41:47 PM): ohhh

18 MAN (7:41:57 PM): that makes sense

19 MAN (7:42:02 PM): so whatcha doing tonight?

20 DEC (7:42:07 PM): nutin

21 DEC (7:42:08 PM): bored

22 DEC (7:42:09 PM): u

23 DEC (7:42:13 PM): whts ur name

24 MAN (7:42:17 PM): (are you looking for a friend? boyfriend? fun?)

25 MAN (7:42:21 PM): i am Mike :)

26 DEC (7:42:27 PM): hi mike

27 MAN (7:42:29 PM): from quiet leesburg

28 DEC (7:42:37 PM): lol

29 MAN (7:42:37 PM): moved here from florida

30 DEC (7:42:41 PM): kewl

31 MAN (7:42:42 PM): and i miss it

32 DEC (7:42:47 PM): never ben there

33 DEC (7:42:50 PM): what part

$34 \operatorname{MAN}(7: 42: 58 \mathrm{PM})$ : east coast

35 DEC (7:42:59 PM): brb gotta pe

36 MAN (7:43:03 PM): daytona beach and port st lucis

37 MAN (7:43:06 PM): lucie

\section{Chat \#62}

1 MAN (07/20/06 7:04:54 PM): hi

2 DEC (07/20/06 7:05:00 PM): hi

3 MAN (07/20/06 7:05:05 PM): how r u

4 DEC (07/20/06 7:06:01 PM): i'm good

5 DEC (07/20/06 7:06:04 PM): a/s/1

6 MAN (07/20/06 7:06:11 PM): $35 \mathrm{~m} \mathrm{fl}$

7 MAN (07/20/06 7:06:11 PM): u

8 DEC (07/20/06 7:06:24 PM): 15/f/fl

9 MAN (07/20/06 7:06:30 PM): oh

10 MAN (07/20/06 7:06:38 PM): what $r u$ up to

11 DEC (07/20/06 7:06:49 PM): nothing much

12 DEC (07/20/06 7:06:54 PM): bored out of my head really

13 MAN (07/20/06 7:07:14 PM): awww danr it

14 MAN (07/20/06 7:07:17 PM): makes 2 of us

15 MAN (07/20/06 7:07:57 PM): so what $r$ we gonna do

16 DEC (07/20/06 7:07:58 PM): my name is cara

17 MAN (07/20/06 7:08:09 PM): nice to meet u cara

18 MAN (07/20/06 7:08:11 PM): nick

$19 \operatorname{DEC}(07 / 20 / 06$ 7:08:14 PM): hi nick

20 DEC (07/20/06 7:08:19 PM): my name is really maddison

21 DEC (07/20/06 7:08:23 PM): but,

22 DEC (07/20/06 7:08:33 PM): that's my mom's name

23 DEC (07/20/06 7:08:40 PM): so i go by my middle name

24 MAN (07/20/06 7:08:49 PM): oh

25 MAN (07/20/06 7:08:58 PM): i like cara better

26 MAN (07/20/06 7:09:02 PM): short and sweet

27 DEC (07/20/06 7:09:06 PM): just like me 
28 DEC (07/20/06 7:09:13 PM): lol

$29 \operatorname{MAN}(07 / 20 / 06$ 7:09:21 PM): well how do i know u r sweet w/o tasting? lol

30 DEC (07/20/06 7:09:34 PM): i don't know really

31 DEC (07/20/06 7:09:43 PM): i guess i don't

32 DEC (07/20/06 7:09:46 PM): :(

33 MAN (07/20/06 7:10:01 PM): awwww i guess i'll just have to try it

34 DEC (07/20/06 7:10:41 PM): whatcha thinking?

35 MAN (07/20/06 7:10:52 PM): about us

36 MAN (07/20/06 7:10:53 PM): lol

37 DEC (07/20/06 7:11:35 PM): really?

38 MAN (07/20/06 7:11:50 PM): $y$ what do u have in mind

39 MAN (07/20/06 7:12:02 PM): someone else?

\section{Chat \#63}

1 MAN (09/08/06 6:39:36 PM)hello

2 DEC (09/08/06 7:26:55 PM): hi

3 MAN (09/08/06 8:50:26 PM)whats up

4 DEC (09/08/06 9:35:37 PM): hi

5 MAN (09/08/06 9:35:54 PM): whats up

6 DEC (09/08/06 9:36:00 PM): nuthin

7 DEC (09/08/06 9:36:04 PM): hang on a sec

8 MAN (09/08/06 9:36:13 PM): ok....

9 MAN (09/08/06 9:36:18 PM): you've had me on hold alll day though

10 MAN (09/08/06 9:36:22 PM): i dont think im interested now

11 MAN (09/08/06 9:36:24 PM): take care

12 DEC (09/08/06 9:36:32 PM): fine

13 MAN (09/08/06 9:44:56 PM): bye

14 MAN (09/08/06 9:45:04 PM): i hate rude inconsiderate women

15 DEC (09/08/06 9:45:32 PM): im not im sorry

16 MAN (09/08/06 9:45:43 PM): where are u from

17 DEC (09/08/06 9:45:55 PM): cali

18 MAN (09/08/06 9:46:03 PM): where in cali

19 DEC (09/08/06 9:46:28 PM): long beach

20 MAN (09/08/06 9:46:36 PM): oh ok

21 DEC (09/08/06 9:46:42 PM): asl?

22 MAN (09/08/06 9:46:48 PM): 28 male carson

23 DEC (09/08/06 9:46:54 PM): kewl

24 MAN (09/08/06 9:47:00 PM): how old are u

25 DEC (09/08/06 9:47:06 PM): 13

26 MAN (09/08/06 9:47:11 PM): 13?

27 DEC (09/08/06 9:47:17 PM): yea

28 DEC (09/08/06 9:47:48 PM): hello

29 MAN (09/08/06 9:47:52 PM): hi

30 MAN (09/08/06 9:48:02 PM): do u have a cam

31 DEC (09/08/06 9:48:08 PM): no lol

32 MAN (09/08/06 9:48:11 PM): :)

33 DEC (09/08/06 9:48:13 PM): wha u look like

34 MAN (09/08/06 9:48:21 PM): like a guy :P

35 MAN (09/08/06 9:48:25 PM): got any older sisters?

Chat \#64

1 MAN (03/27/07 4:27:24 PM): hi colie

2 DEC (03/27/07 4:27:40 PM): hi :) asl?

3 MAN (03/27/07 4:27:58 PM): $\mathrm{m} / 38$ paramus

$4 \operatorname{DEC}(03 / 27 / 07$ 4:28:53 PM): oh cool im $13 \mathrm{f} \mathrm{nj}$ 

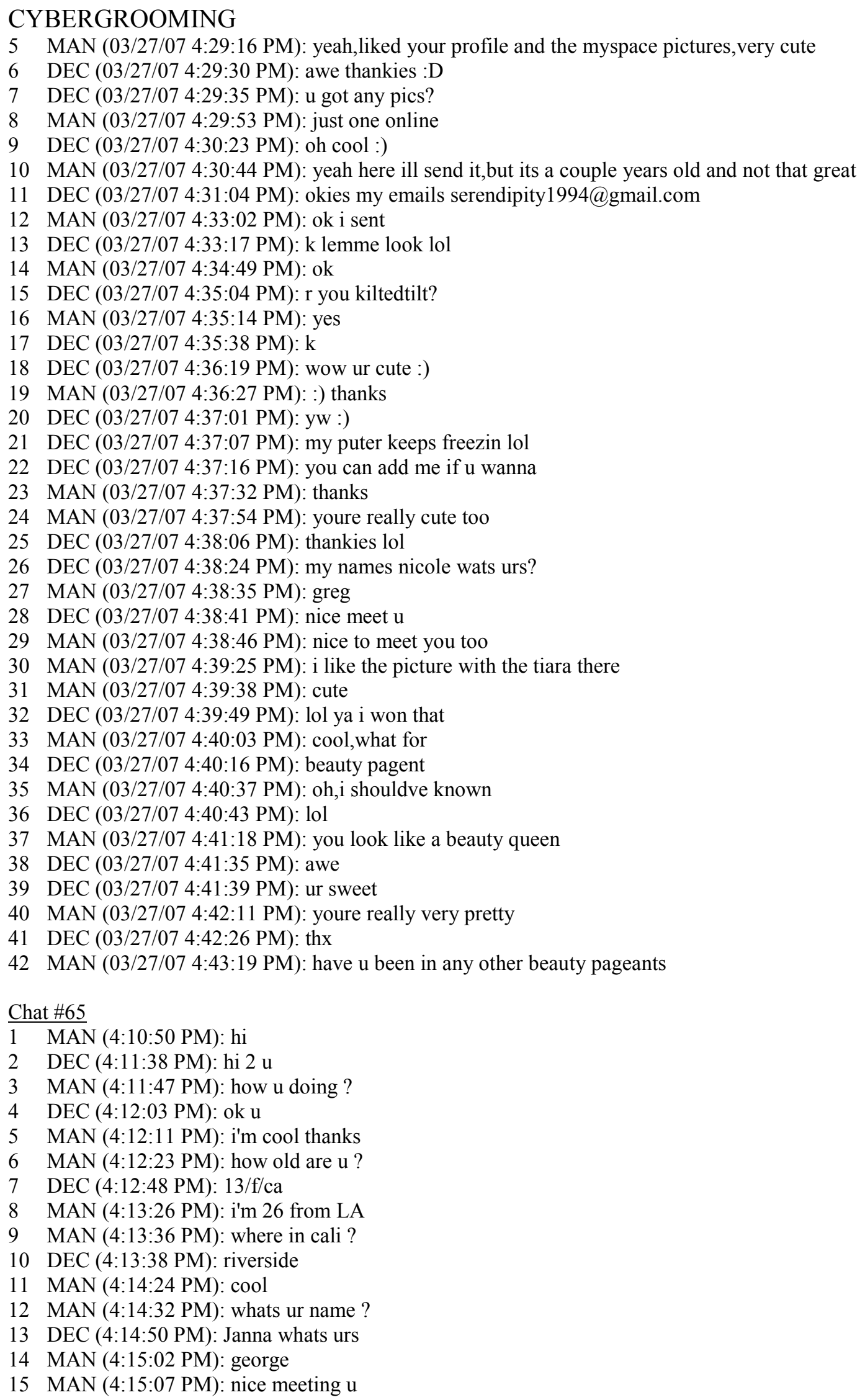
16 MAN (4:15:31 PM): are u white ?

17 DEC (4:15:38 PM): yeah

18 MAN (4:15:48 PM): i'm mexican

19 MAN (4:15:54 PM): do u like mexican guys ?

20 DEC (4:16:17 PM): it dont matter as long as they $r$ nice

21 MAN (4:16:34 PM): cool

22 MAN (4:16:52 PM): who do u live with ?

23 DEC (4:17:15 PM): my mom

$24 \operatorname{MAN}(4: 17: 42 \mathrm{PM})$ : do u have a pic ?

25 DEC (4:18:00 PM): not yet

26 MAN (4:18:13 PM): how do u look like?

27 DEC (4:19:17 PM): like my avatar pretty mcuch

28 MAN (4:20:28 PM): hahaha

29 DEC (4:21:17 PM): lol yeah its kinda lame

30 DEC (4:21:23 PM): but i dont got any pics on here yet

31 MAN (4:21:48 PM): its cool

32 MAN (4:21:55 PM): u got a boyfriend ?

\section{Chat \#66}

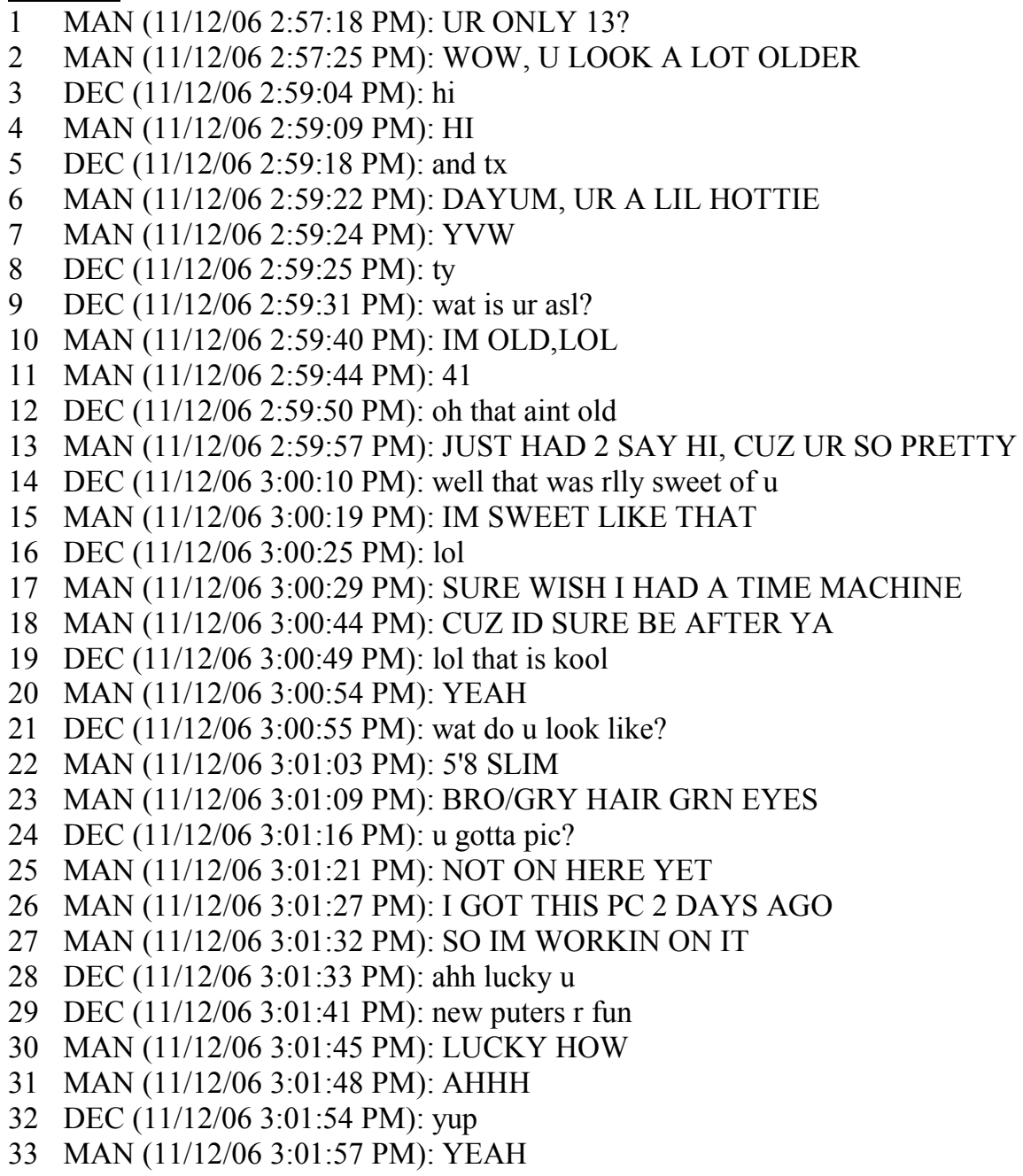

Chat \#67

1 MAN (9:13:02 AM): hi 


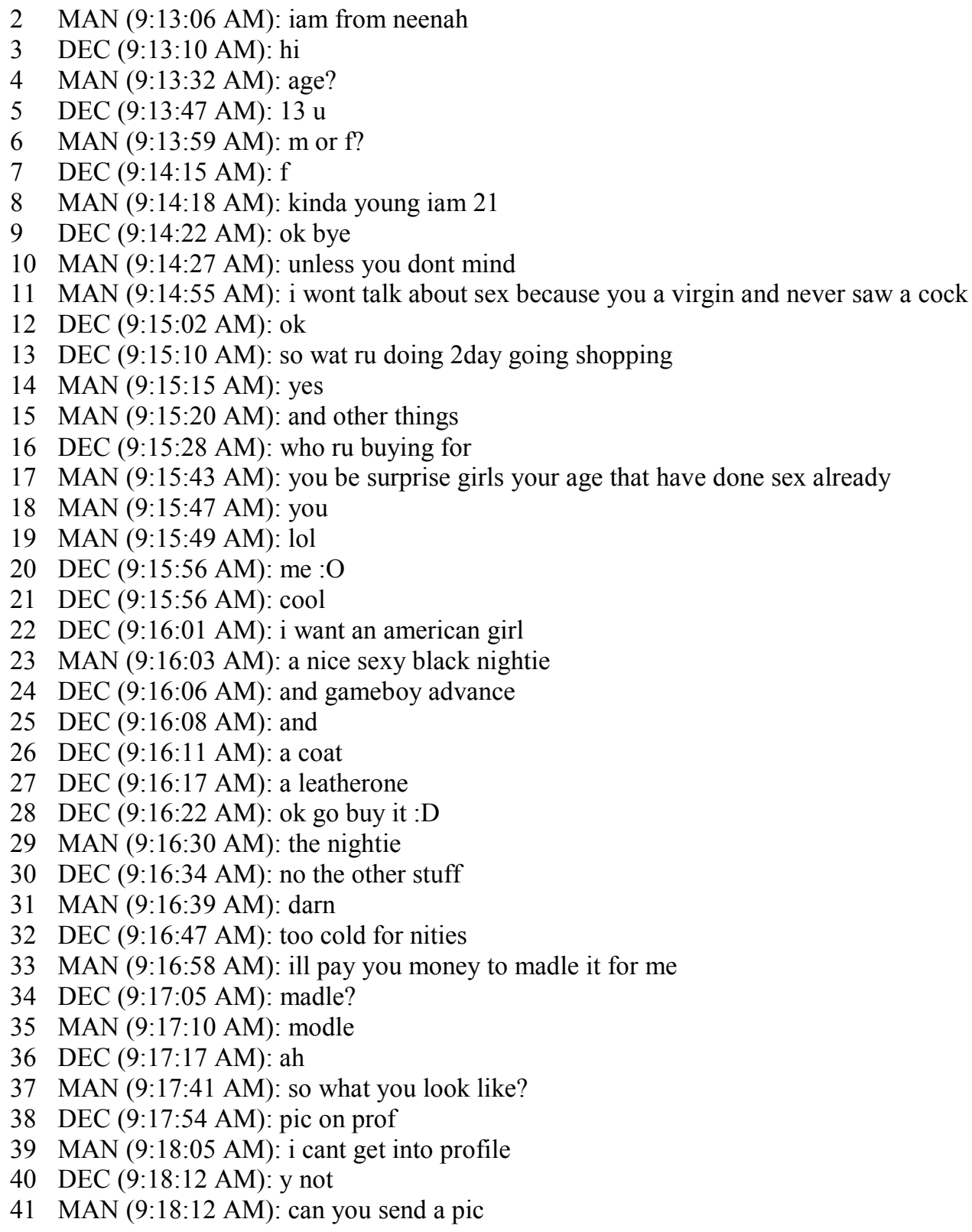


14 MAN (03/25/07 5:41:56 PM): where ya from?

15 DEC (03/25/07 5:42:01 PM): nj

16 DEC (03/25/07 5:42:04 PM): u?

17 MAN (03/25/07 5:42:22 PM): swpa..:(

18 DEC (03/25/07 5:42:41 PM): o

19 MAN (03/25/07 5:43:03 PM): i bet u got a bf anyway

20 DEC (03/25/07 5:44:29 PM): na not now

21 MAN (03/25/07 5:44:40 PM): get out ur a sweetie

22 DEC (03/25/07 5:44:48 PM): aw ty

$23 \operatorname{MAN}(03 / 25 / 07$ 5:44:51 PM): yw

24 DEC (03/25/07 5:44:52 PM): how old $\mathrm{r} u$ ?

25 MAN (03/25/07 5:45:32 PM): too old...

26 DEC (03/25/07 5:46:59 PM): like?

27 MAN (03/25/07 5:47:14 PM): 28

28 DEC (03/25/07 5:47:24 PM): o lol

29 DEC (03/25/07 5:47:26 PM): im 14

30 MAN (03/25/07 5:47:34 PM): dang u look so sweet

31 MAN (03/25/07 5:47:57 PM): well take care....nice chattin

32 DEC (03/25/07 5:49:44 PM): u goin?

33 MAN (03/25/07 5:50:01 PM): well prob shouldnt be talkin to ya

34 MAN (03/25/07 5:50:34 PM): what do ya like to do for fun????

35 DEC (03/25/07 5:50:53 PM): mostly hang out wit freinds $n$ watevers fun

36 DEC (03/25/07 5:50:57 PM): how bout $\mathrm{u}$ ?

37 MAN (03/25/07 5:51:31 PM): hang out n party.....listen to music......movies...sex

38 DEC (03/25/07 5:51:40 PM): lol

$39 \operatorname{MAN}(03 / 25 / 07$ 5:51:47 PM): what?

Chat \#69

1 MAN (07/15/06 9:32:20 PM): hello how u doing

2 DEC (07/15/06 9:33:59 PM): hi

3 MAN (07/15/06 9:34:29 PM): so what $u$ up to tonight

$4 \quad \operatorname{DEC}(07 / 15 / 06$ 9:34:59 PM): nothin much

5 MAN (07/15/06 9:35:04 PM): same here

6 MAN (07/15/06 9:35:10 PM): what ur age

7 DEC (07/15/06 9:35:15 PM): im 15

8 DEC (07/15/06 9:35:17 PM): u?

9 MAN (07/15/06 9:35:26 PM): 23

10 MAN (07/15/06 9:35:32 PM): where $r u$ from in ga

11 DEC (07/15/06 9:35:45 PM): im kinda near columbus

12 DEC (07/15/06 9:35:47 PM): u?

13 MAN (07/15/06 9:35:59 PM): albany

$14 \operatorname{MAN}(07 / 15 / 06$ 9:36:05 PM): where is ur pic

$15 \operatorname{DEC}(07 / 15 / 06$ 9:36:14 PM): thers 1 in my prof

$16 \operatorname{MAN}(07 / 15 / 06$ 9:36:57 PM): u r cute

17 DEC (07/15/06 9:37:00 PM): ty

18 MAN (07/15/06 9:37:07 PM): do u have a b/f

19 DEC (07/15/06 9:37:15 PM): no not now

20 MAN (07/15/06 9:37:33 PM): hard to belive as cute as u r

21 DEC (07/15/06 9:37:42 PM): lol ty

22 MAN (07/15/06 9:38:07 PM): what do u do for fun

23 DEC (07/15/06 9:38:22 PM): i play guitar $\mathrm{n}$ swim

24 DEC (07/15/06 9:38:25 PM): u?

25 MAN (07/15/06 9:38:30 PM): oh ok nice

26 MAN (07/15/06 9:38:34 PM): i work out alot

27 MAN (07/15/06 9:38:45 PM): i am 6"1 200 brw eyes dark hair cute smile 
28 DEC (07/15/06 9:38:56 PM): do u have a pic?

$29 \operatorname{MAN}(07 / 15 / 06$ 9:39:31 PM): not yet

30 MAN (07/15/06 9:39:38 PM): so u like older guys

31 DEC (07/15/06 9:39:55 PM): thers bad or nice guys all ages

32 MAN (07/15/06 9:40:32 PM): u ever date someone my ate

33 DEC (07/15/06 9:40:42 PM): not yet lol

34 MAN (07/15/06 9:41:10 PM): love to kiss u

35 DEC (07/15/06 9:41:25 PM): wow lol

36 MAN (07/15/06 9:41:35 PM): did i offend u

\section{Chat \#70}

1 MAN (03/23/07 1:03:31 PM): how you doing today?

2 DEC (03/23/07 1:04:55 PM): hey

3 DEC (03/23/07 1:04:57 PM): asl

4 DEC (03/23/07 1:05:01 PM): 13/f/ky here

5 MAN (03/23/07 1:05:15 PM): 22/m/cincy......am i too old for u?

6 DEC $(03 / 23 / 07$ 1:05:21 PM): no

7 MAN (03/23/07 1:05:27 PM): cool

8 DEC (03/23/07 1:05:30 PM): am i 2 yung 4 u ?

9 MAN (03/23/07 1:06:07 PM): idk, i dont know u yet....age means nothing to me, sexy is sexy, fun is fun, and most importantly mature is mature...if $u$ act older i see $u$ as older

10 DEC (03/23/07 1:06:37 PM): k

11 MAN (03/23/07 1:07:31 PM): so u mature?

12 MAN (03/23/07 1:08:51 PM): ?

13 DEC (03/23/07 1:09:12 PM): i think so

$14 \operatorname{MAN}(03 / 23 / 07$ 1:09:20 PM): very cool

15 MAN (03/23/07 1:09:23 PM): u have any more pics?

16 DEC (03/23/07 1:09:45 PM): n my ms page

17 MAN (03/23/07 1:11:27 PM): @-) damn you are sexy

18 DEC (03/23/07 1:11:35 PM): lol ty

$19 \operatorname{MAN}(03 / 23 / 07$ 1:11:45 PM): why no man?

$20 \operatorname{DEC}(03 / 23 / 07$ 1:12:11 PM): i duno cant find 1 that nice i gues

21 MAN (03/23/07 1:12:26 PM): well what kinda guys u like?

$22 \operatorname{DEC}(03 / 23 / 07$ 1:13:11 PM): nice 1

23 MAN (03/23/07 1:13:29 PM): nice isnt very specific

24 DEC (03/23/07 1:13:59 PM): 1 that treet me good $\mathrm{n}$ nice 2 me $\mathrm{n}$ not play head gams

25 MAN (03/23/07 1:14:18 PM): well i havent been eliminated yet...lol

26 DEC (03/23/07 1:14:26 PM): lol

27 MAN (03/23/07 1:15:21 PM): whats the oldest guy u have dated?

28 DEC (03/23/07 1:15:38 PM): 16

29 MAN (03/23/07 1:15:53 PM): how long ago was that?

30 DEC (03/23/07 1:16:30 PM): las summer

31 DEC (03/23/07 1:16:35 PM): what yur name

32 DEC (03/23/07 1:16:39 PM): mines Kelly

33 MAN (03/23/07 1:16:53 PM): Nice to meet you Kelly. I am Gary

34 DEC (03/23/07 1:17:05 PM): nice 2 meet u 2

35 MAN (03/23/07 1:17:11 PM): :)

36 MAN (03/23/07 1:17:43 PM): so what would u say your limit is as far as age you want your bf to be?

37 DEC (03/23/07 1:18:08 PM): i duno i like older but not like $90 \mathrm{n}$ all rinkly $\mathrm{n}$ shit lol

38 MAN (03/23/07 1:18:31 PM): lol....may i ask why u like older?

$39 \operatorname{DEC}(03 / 23 / 07$ 1:18:46 PM): i duno cuz there more matur

40 MAN (03/23/07 1:18:58 PM): cool

Chat \#71

1 MAN (09/01/06 11:40:46 PM): hi 
MAN (09/01/06 11:40:52 PM): how u doin ?

DEC (09/01/06 11:41:04 PM): hi

MAN (09/01/06 11:41:10 PM): asl ?

DEC (09/01/06 11:41:19 PM): 13/f/cali u?

MAN (09/01/06 11:41:32 PM): male 17 los angeles

7 MAN (09/01/06 11:41:37 PM): do u have a picture ?

8 DEC (09/01/06 11:42:01 PM): on my profile

9 DEC (09/01/06 11:42:20 PM): ur 2 young tho

10 MAN (09/01/06 11:42:20 PM): wow, u r a hot cutee

11 DEC (09/01/06 11:42:29 PM): i like older guys

$12 \operatorname{MAN}(09 / 01 / 06$ 11:42:46 PM): take a look at my pic

13 MAN (09/01/06 11:42:55 PM): sorry i mistyped

14 MAN (09/01/06 11:42:58 PM): i am 24

15 DEC (09/01/06 11:43:01 PM): relly?

16 MAN (09/01/06 11:43:12 PM): yeah, i have myspace account too

17 MAN (09/01/06 11:43:21 PM): u have nice eyes

18 DEC (09/01/06 11:43:26 PM): ty

$19 \operatorname{MAN}(09 / 01 / 06$ 11:43:46 PM): and tight hair, very amazing indeed

20 DEC (09/01/06 11:43:52 PM): ty

21 DEC (09/01/06 11:43:55 PM): ur relly 24?

22 MAN (09/01/06 11:44:01 PM): yeah, i swear

23 MAN (09/01/06 11:44:08 PM): my birthday in march 11, 1982

24 DEC (09/01/06 11:44:13 PM): kewl

$25 \operatorname{MAN}(09 / 01 / 06$ 11:44:17 PM): i am pieces

26 MAN (09/01/06 11:44:30 PM): what do like about guys my age ?

27 DEC (09/01/06 11:44:39 PM): ur not a guy my age lol

28 DEC (09/01/06 11:44:49 PM): guys my age $r$ stoopid

29 DEC (09/01/06 11:45:01 PM): $\mathrm{u}$ in cali?

30 MAN (09/01/06 11:45:14 PM): lool

31 MAN (09/01/06 11:45:20 PM): yeah sweety

32 MAN (09/01/06 11:45:27 PM): what part of cali $r u$ in ?

33 DEC (09/01/06 11:45:31 PM): near LA

34 MAN (09/01/06 11:45:35 PM): cool

\section{Chat \#72}

1 MAN (05/20/07 12:53:59 PM): hi

2 MAN (05/20/07 12:54:04 PM): u r cute

3 DEC (05/20/07 12:54:09 PM): hey

4 DEC (05/20/07 12:54:10 PM): ty

5 MAN (05/20/07 12:54:15 PM): yw

6 MAN $(05 / 20 / 07$ 1:00:19 PM): what $r$ u up to

7 DEC (05/20/07 1:00:29 PM): nothin

$8 \operatorname{MAN}(05 / 20 / 07$ 1:00:42 PM): same here

9 DEC (05/20/07 1:01:00 PM): im alyce

10 MAN (05/20/07 1:01:08 PM): i am billy

11 DEC (05/20/07 1:01:13 PM): hey

12 DEC (05/20/07 1:01:21 PM): how old $\mathrm{r} u$

13 MAN (05/20/07 1:01:31 PM): 25

$14 \operatorname{MAN}(05 / 20 / 07$ 1:01:40 PM): how old $\mathrm{r} \mathrm{u}$

15 DEC (05/20/07 1:01:46 PM): 13

$16 \operatorname{MAN}(05 / 20 / 07$ 1:02:28 PM): sorry

17 MAN (05/20/07 1:02:42 PM): u look older

$18 \operatorname{DEC}(05 / 20 / 07$ 1:02:50 PM): ty

19 DEC (05/20/07 1:03:48 PM): u look younger

20 MAN (05/20/07 1:03:54 PM): thank u 
21 MAN (05/20/07 1:03:59 PM): u r sweeet

22 MAN (05/20/07 1:04:30 PM): and u $r$ a beautiful girl

23 MAN (05/20/07 1:04:36 PM): i wish i was younger

24 MAN (05/20/07 1:04:38 PM): lol

25 DEC (05/20/07 1:05:04 PM): aww tyvm

26 MAN (05/20/07 1:05:18 PM): yw

27 MAN (05/20/07 1:18:14 PM): did u leave

28 DEC $(05 / 20 / 07$ 1:18:22 PM): na im here

29 DEC (05/20/07 1:18:27 PM): i thought u left

30 MAN (05/20/07 1:18:31 PM): nope

31 MAN (05/20/07 1:18:38 PM): i would never leave u

32 MAN (05/20/07 1:18:44 PM): >:D<

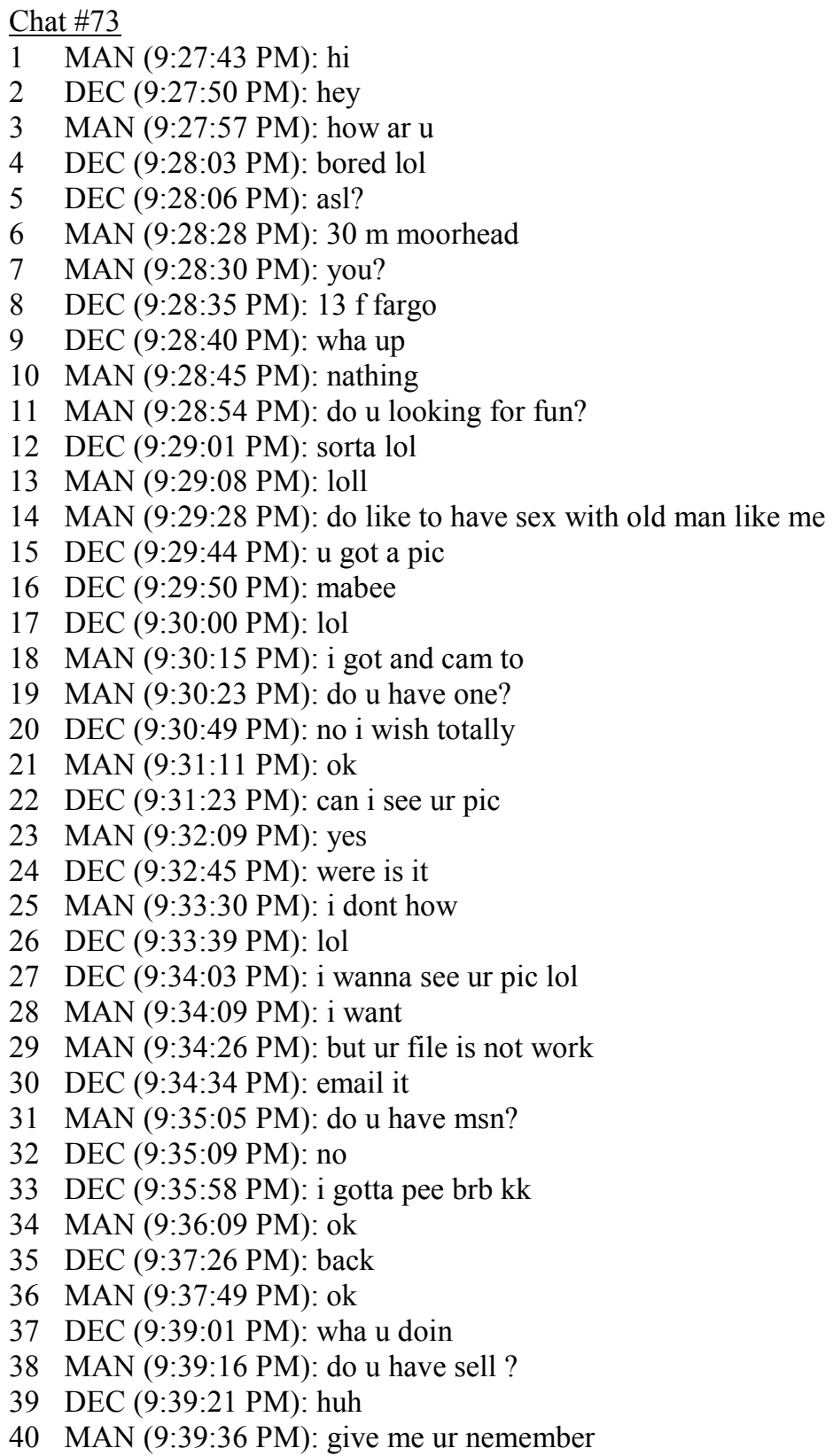


Chat \#74

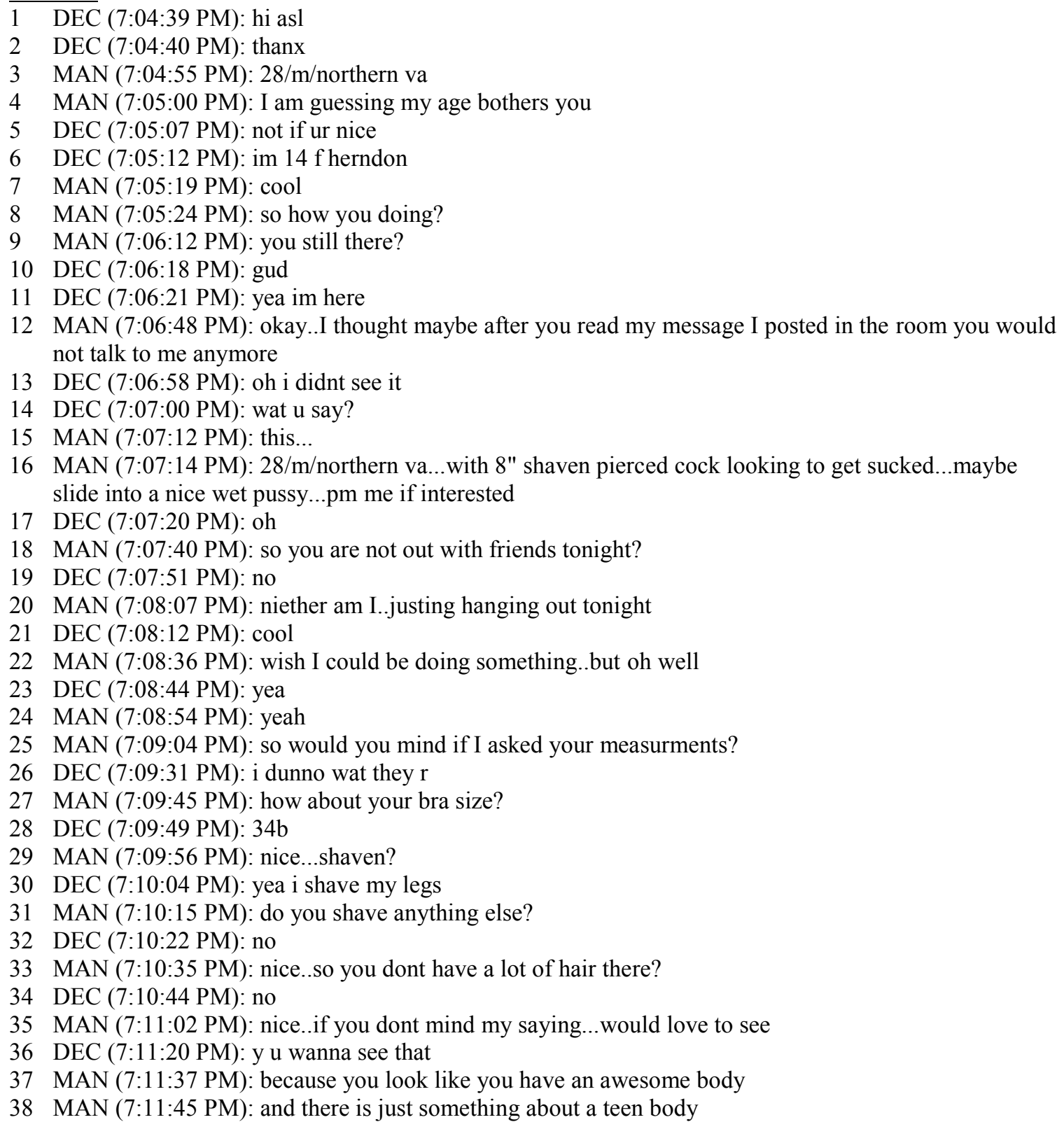

\section{Chat \#75}

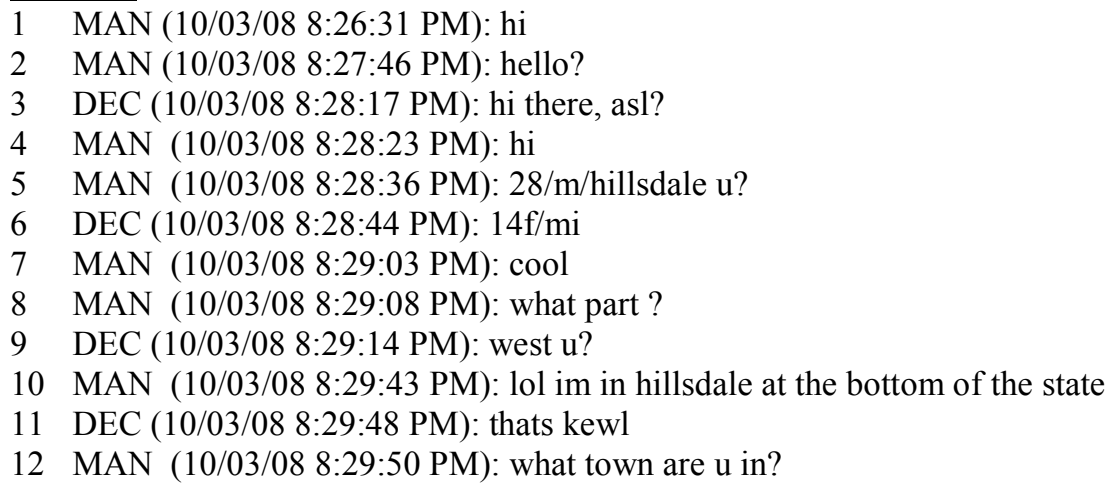


13 DEC (10/03/08 8:30:00 PM): i dont even know u yet

14 MAN (10/03/08 8:30:21 PM): oh sorry

15 DEC (10/03/08 8:31:00 PM): so whats up

16 MAN (10/03/08 8:31:22 PM): not much just bored u?

17 DEC (10/03/08 8:31:48 PM): yah same

18 MAN (10/03/08 8:32:18 PM): so are u single?

19 DEC (10/03/08 8:32:21 PM): yah

20 MAN (10/03/08 8:32:48 PM): me too

$21 \operatorname{DEC}(10 / 03 / 08$ 8:32:53 PM): thats kewl

22 MAN (10/03/08 8:33:26 PM): do u have a pic?

23 DEC (10/03/08 8:33:31 PM): yah

24 MAN (10/03/08 8:33:55 PM): may i see it mine is in my pro

25 DEC (10/03/08 8:34:01 PM): yah hold on

26 MAN (10/03/08 8:35:04 PM): wow ur a lil hottie

27 DEC (10/03/08 8:35:09 PM): hehe thx

28 MAN (10/03/08 8:35:21 PM): so how come ur single?

29 DEC (10/03/08 8:35:31 PM): eh just havent found the right guy yet i guess

30 MAN (10/03/08 8:35:57 PM): lol me too

31 DEC (10/03/08 8:36:03 PM): lol u looking for a guy?

32 MAN (10/03/08 8:36:19 PM): lol no girl

33 DEC (10/03/08 8:36:23 PM): lol thats better

34 MAN (10/03/08 8:36:36 PM): maybe im looking at her

35 DEC (10/03/08 8:36:47 PM): haha u think so?

36 MAN (10/03/08 8:37:00 PM): who knows maybe

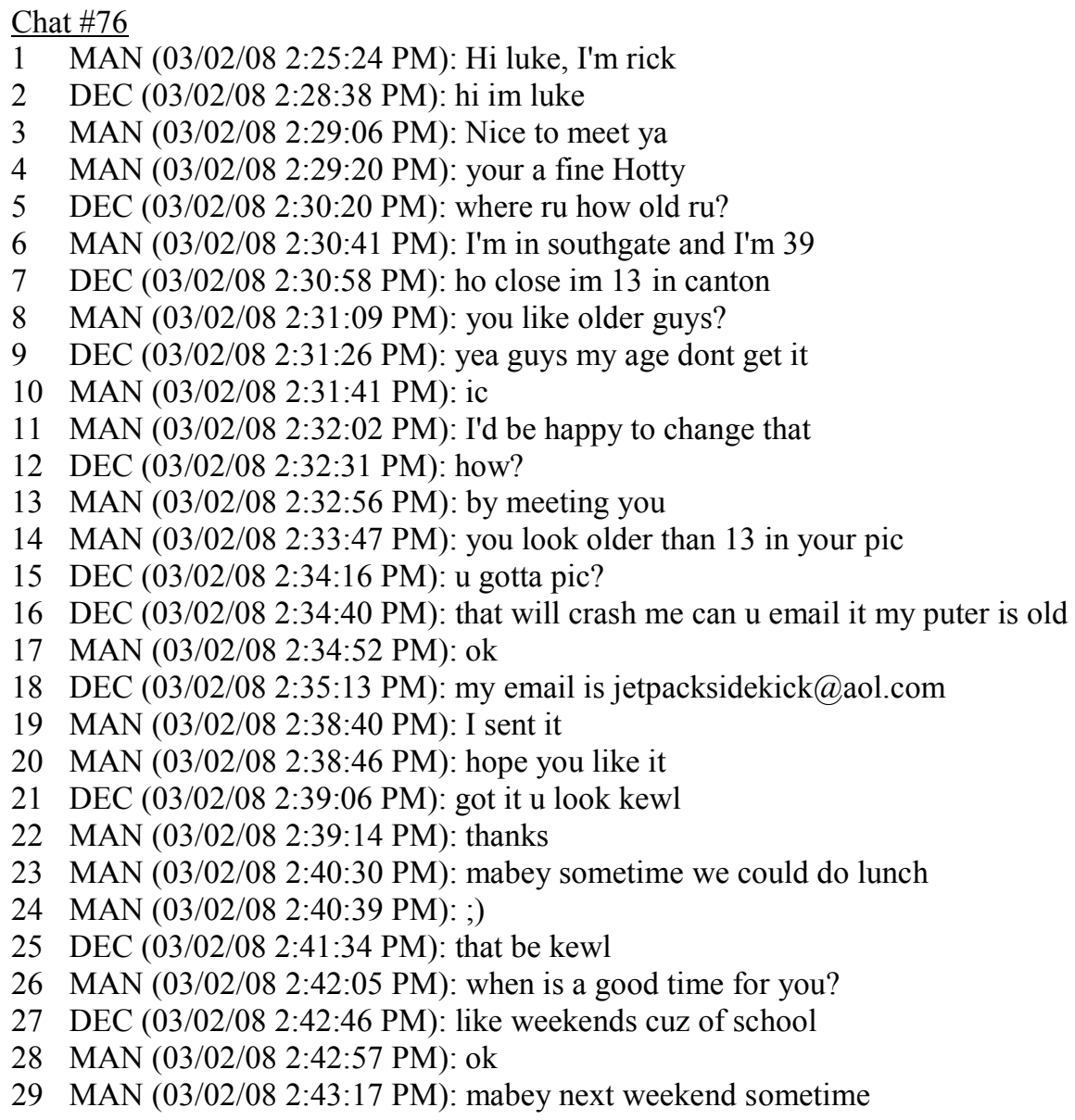


30 DEC (03/02/08 2:43:29 PM): that b kewl what u wanna do?

31 MAN (03/02/08 2:43:45 PM): it's up to you

32 MAN (03/02/08 2:44:03 PM): I'll do anything you want

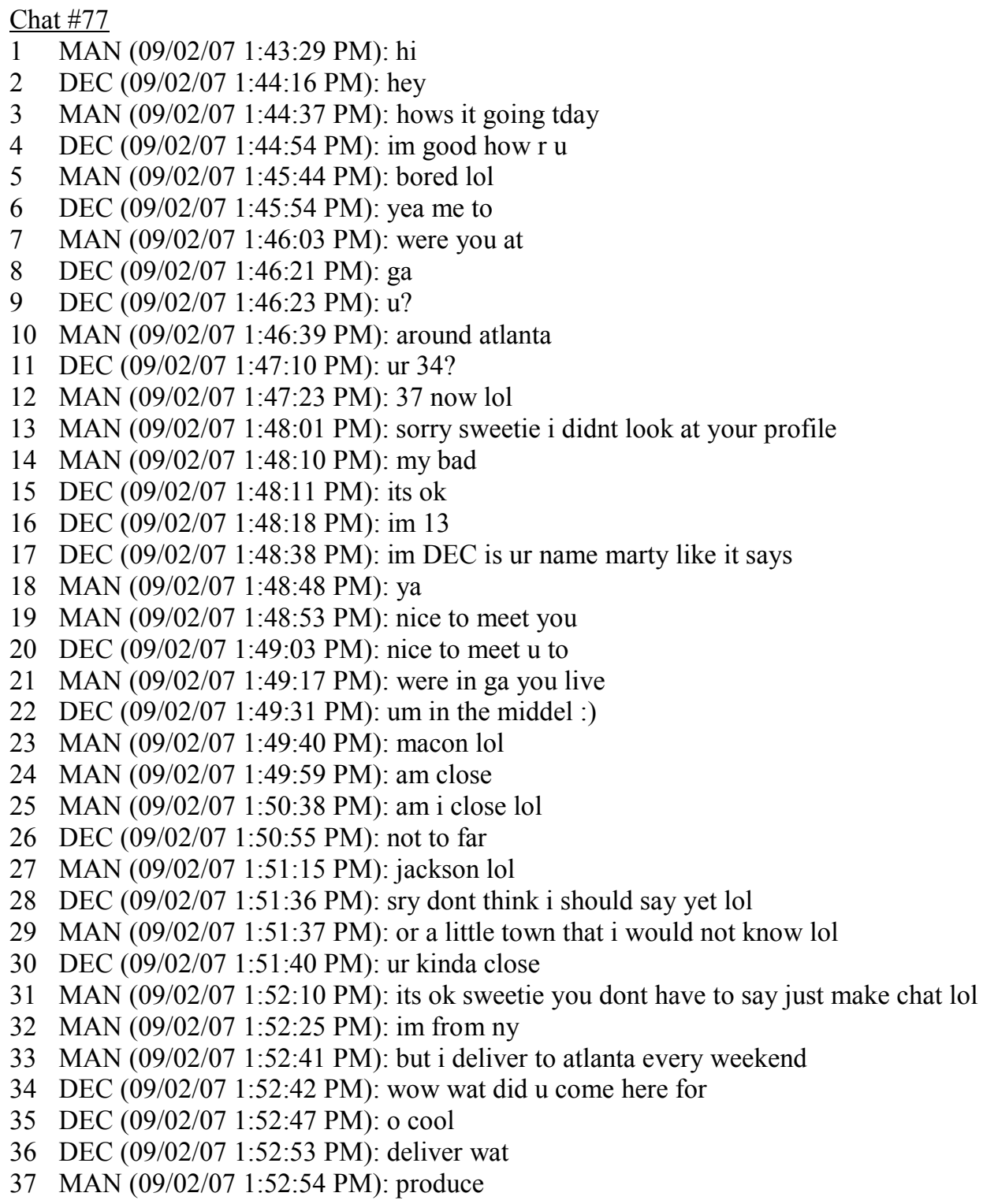

Chat \#78

1 MAN [12:43 PM]: such a cute boi, why so negative

2 DEC [12:43 PM]: live sucks

3 MAN [12:44 PM]: so do lots of things kid, dont let it get YOU down

4 DEC [12:45 PM]: i hate ny and being poor and gay

5 MAN [12:46 PM]: sorry life suckss, but get over it

6 DEC [12:46 PM]: if i got a rich dad i be happy

7 MAN [12:47 PM]: reely, i can make YOu reel happy boi

8 DEC [12:47 PM]: how?

9 MAN [12:47 PM]: taking YOu into my life and makeing YOU my little fagboi and make YOU adore me and my needs

10 DEC [12:48 PM]: ur profi says ur a boy scot dude they not rich 
11 MAN [12:48 PM]: yeah but i have plenty from an inheritance boi

12 DEC [12:49 PM]: u really a boy scot?

13 DEC [12:50 PM]: ?

14 MAN [12:50 PM]: yes, of course i am

15 DEC [12:50 PM]: thats so gay

16 MAN [12:51 PM]: why, you should see some of those bois naked bodies, waht a turn on ,its hard to not want them

17 DEC [12:51 PM]: they dont got hair

18 MAN [12:52 PM]: well most dont, and there the ons i like to "watch"

19 DEC [12:52 PM]: i like big cocks

20 MAN [12:53 PM]: im into looking at my bois asses

21 DEC [12:53 PM]: <---14 i got hair when i was 11

22 MAN [12:54 PM]: wow relly

23 DEC [12:54 PM]: where u frum?

24 MAN [12:55 PM]: long islnad

25 DEC [12:55 PM]: daym ur close

26 MAN [12:56 PM]: but i only like young bois

27 DEC [12:57 PM]: i no lots of dudes younger then me

28 DEC [1:00 PM]: u there?

29 DEC [1:08 PM]: where u go?

30 MAN [1:08 PM]: wetting up a meeting with rickyboi

31 DEC [1:09 PM]: huh?

32 MAN [1:09 PM]: sorry timing is everything

33 DEC [1:10 PM]: y u say that?

34 MAN [1:11 PM]: casue i wanted YOU fisrst

35 DEC [1:11 PM]: im still here

36 MAN [1:11 PM]: yeah, but ricky is already waiting for me

37 DEC [1:11 PM]: how old he?

38 MAN [1:12 PM]: and he doesnt even know waht $\mathrm{i}$ want, he was so horny he gaveme his adress

39 MAN [1:12 PM]: 15

40 DEC [1:12 PM]: im 14

41 MAN [1:13 PM]: i bet YOU have a reel smooth fag ass, i sure would love to tear it UP

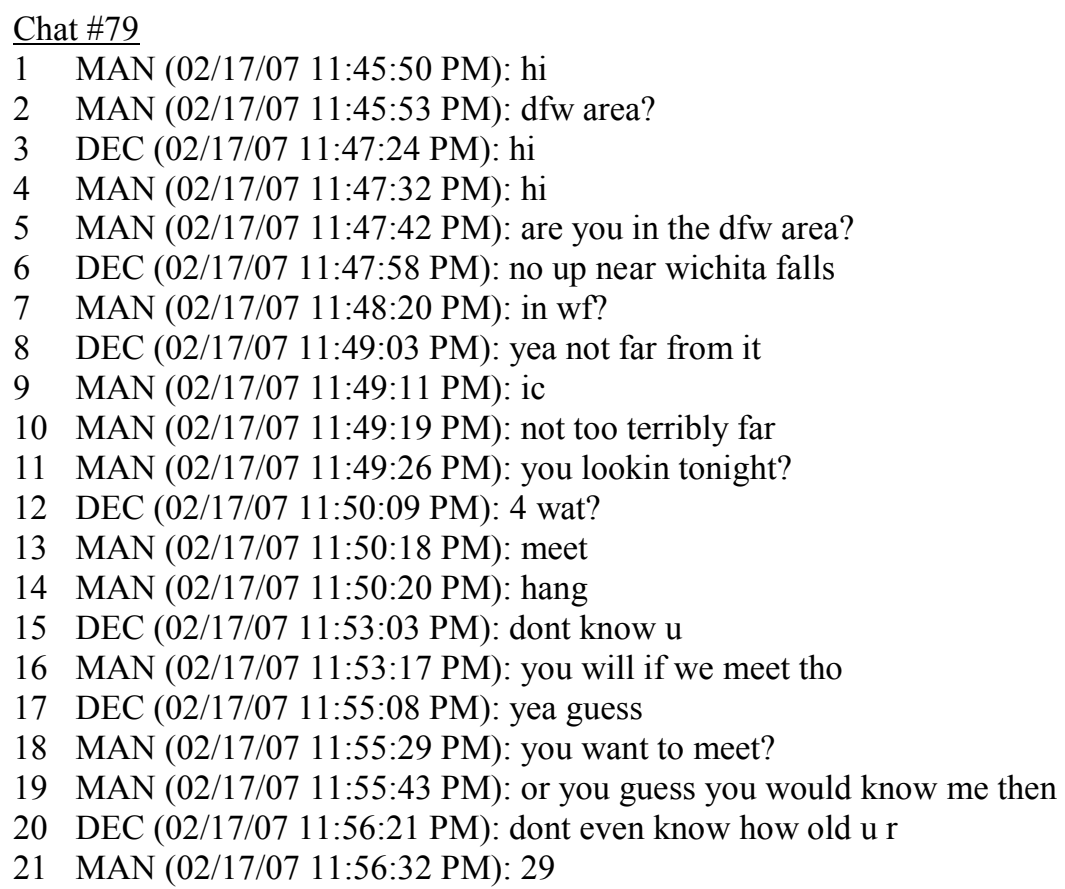


22 DEC (02/17/07 11:57:27 PM): im 15

23 MAN (02/17/07 11:58:01 PM): oic

24 MAN (02/17/07 11:58:14 PM): kinda hard for you to get out then

$25 \operatorname{MAN}(02 / 17 / 07$ 11:58:15 PM): lol

$26 \operatorname{DEC}(02 / 17 / 07$ 11:58:35 PM): yea only when mom is gone

27 MAN (02/17/07 11:58:44 PM): mom gone?

28 MAN (02/17/07 11:58:56 PM): parents are divorced

29 DEC (02/17/07 11:59:15 PM): yea they $r$

30 MAN (02/17/07 11:59:30 PM): she work nights or something

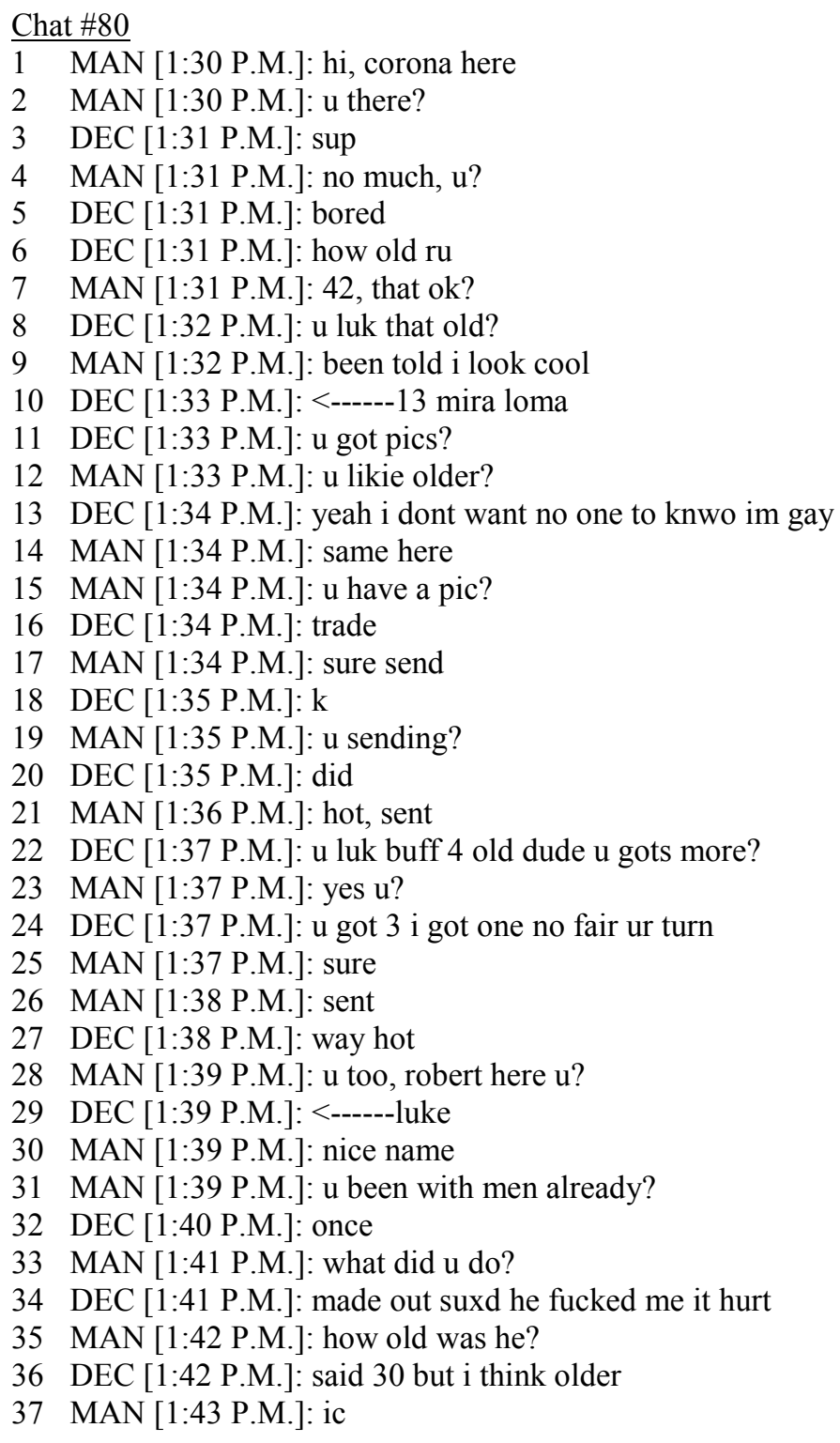

Chat \#81

1 MAN (09/03/06 8:58:41 PM): chirstian and goth?

2 DEC (09/03/06 8:58:53 PM): yeah lol

3 MAN (09/03/06 8:59:20 PM): nice combination

4 MAN (09/03/06 8:59:28 PM): looks like it works well for you

5 MAN (09/03/06 9:00:17 PM): so only black clothes? 
6 DEC (09/03/06 9:00:59 PM): not always but sometimes

7 MAN (09/03/06 9:01:14 PM): fair enough

8 MAN (09/03/06 9:01:45 PM): now out of curiousity do you get a lot of questions regarding being goth and christian

9 DEC (09/03/06 9:02:12 PM): yeah guess it makes ppl wonder

10 MAN (09/03/06 9:02:35 PM): it makes the close minded wonder

11 MAN (09/03/06 9:02:47 PM): and a lot of people of preconceptions of what goth means

12 DEC (09/03/06 9:03:09 PM): yeah exactly

13 MAN (09/03/06 9:03:22 PM): i know just enough to be dangerous :)

14 DEC (09/03/06 9:03:42 PM): lol

15 MAN (09/03/06 9:04:07 PM): still its a nice weekend and a time to relax ... hopefully you are having fun

16 DEC (09/03/06 9:04:25 PM): kinda

17 MAN (09/03/06 9:05:07 PM): only kinda?

18 DEC (09/03/06 9:05:38 PM): gotta go with dad to a party tonite

19 DEC (09/03/06 9:05:43 PM): dont rlly wanna

20 MAN (09/03/06 9:05:52 PM): pretend you are sick;)

21 DEC (09/03/06 9:06:15 PM): he rlly wants us to

22 MAN (09/03/06 9:06:32 PM): ah i know that feeling

23 MAN (09/03/06 9:06:40 PM): when is the party

24 DEC (09/03/06 9:06:48 PM): like 8

25 MAN (09/03/06 9:07:18 PM): are you ready to go?

26 DEC (09/03/06 9:07:30 PM): mostly

27 MAN (09/03/06 9:07:48 PM): what does mostly mean?

28 DEC (09/03/06 9:08:57 PM): almost ready?

29 MAN (09/03/06 9:09:23 PM): yes i kinda figured that out ... i was curious whats left to be done

30 MAN (09/03/06 9:09:24 PM): lol

31 DEC (09/03/06 9:14:10 PM): just brush hair n stuff

32 MAN (09/03/06 9:14:19 PM): ah not so bad

33 MAN (09/03/06 9:14:33 PM): well i hope the party will be fun for you even though you dont think so

\section{Chat \#82}

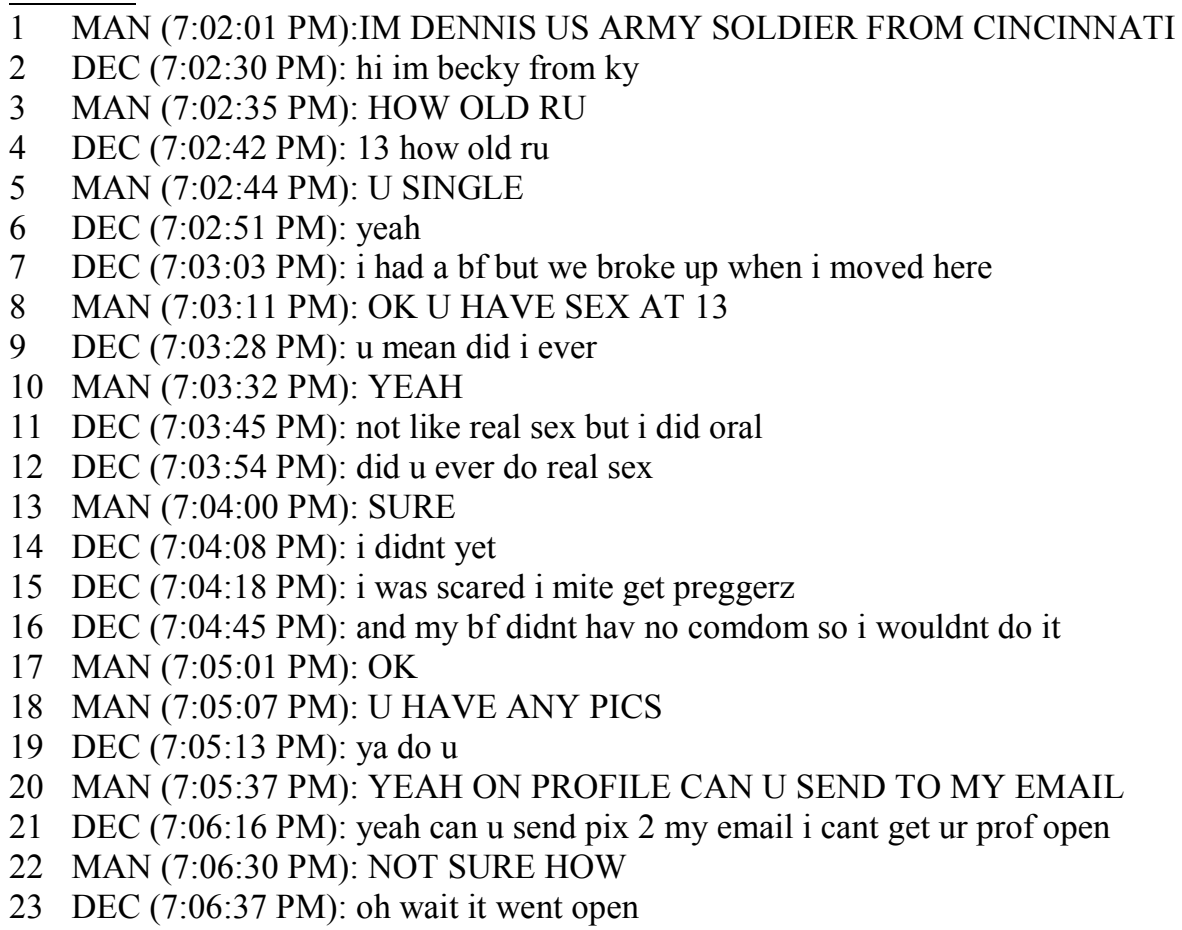


24 DEC (7:06:59 PM): omg ur sooo cute

25 MAN (7:07:04 PM): THANKS

26 MAN (7:07:11 PM): U SEND YOURS

27 DEC (7:07:25 PM): brb im not very good at this yet ill sendf

28 MAN (7:08:48 PM): SURE

29 7:10:17 PM MAN: NICE U HAVE ANY NAKED ONES

30 7:10:54 PM DEC: well no silly

31 7:10:56 PM DEC: hehehe

32 7:11:23 PM DEC: my dad sent me a web cam tho and mom broke it with a hammer and scremed at me

33 7:11:50 PM MAN: WERE U TAKING YOUR CLOTHS OFF FOR PEOPLE

34 7:12:09 PM DEC: no i was just talkin to dad and she hatez him

35 7:12:24 PM MAN: OH SO U DIDNT GET DIRTY ON CAM

36 7:12:40 PM DEC: i only had it 2 dayz b4 she found it

37 7:12:45 PM MAN: OH

38 7:12:54 PM MAN: SO U HAVE BIG TITS

39 7:13:01 PM DEC: no

40 7:13:11 PM MAN: U SHAVE

41 7:13:39 PM DEC: yeah but i dont have much

42 7:13:46 PM MAN: OK

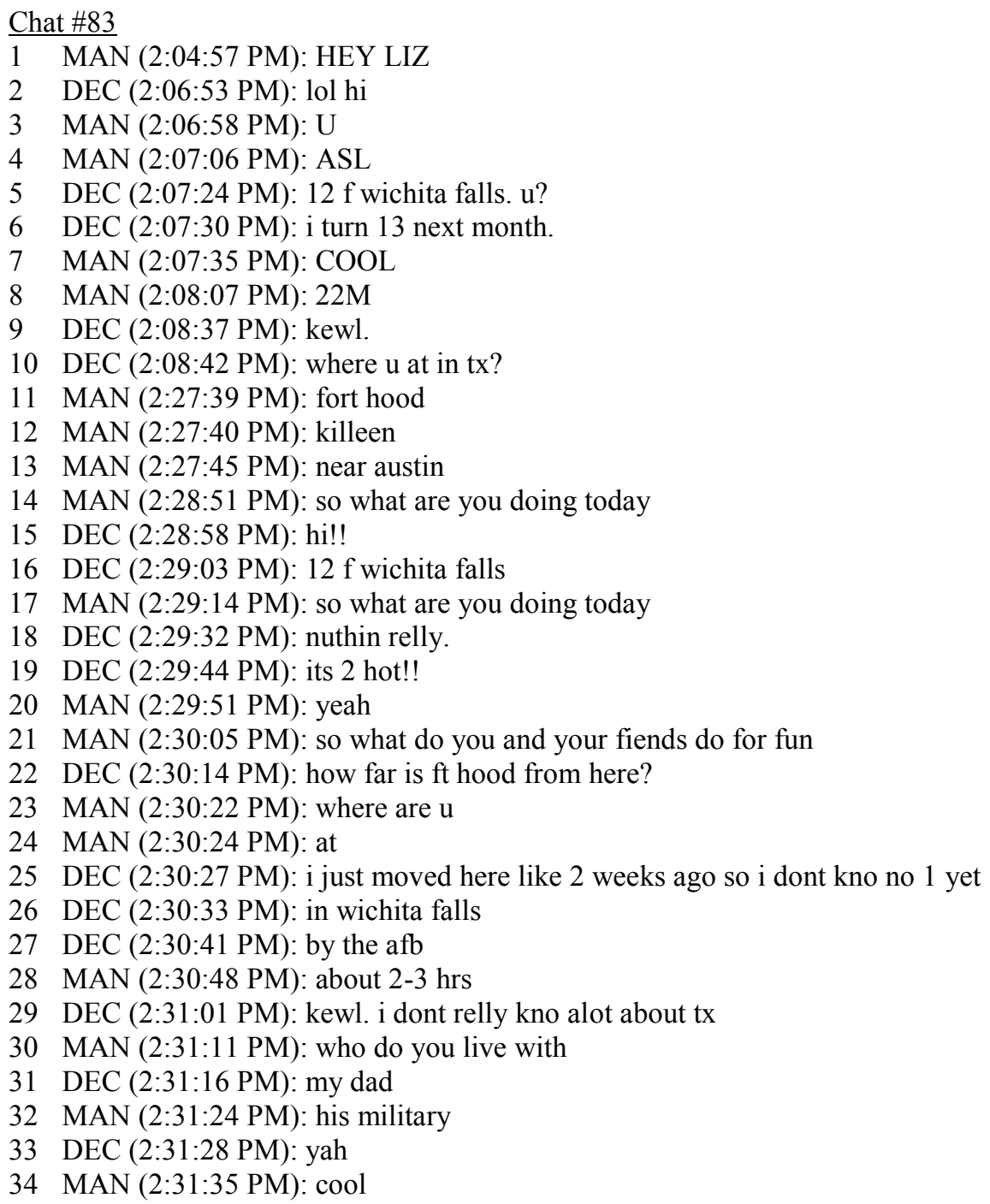


35 MAN (2:31:41 PM): what about your mom

36 DEC (2:31:50 PM): she left us when i was like 4

37 MAN (2:31:58 PM): oh i;m sorry

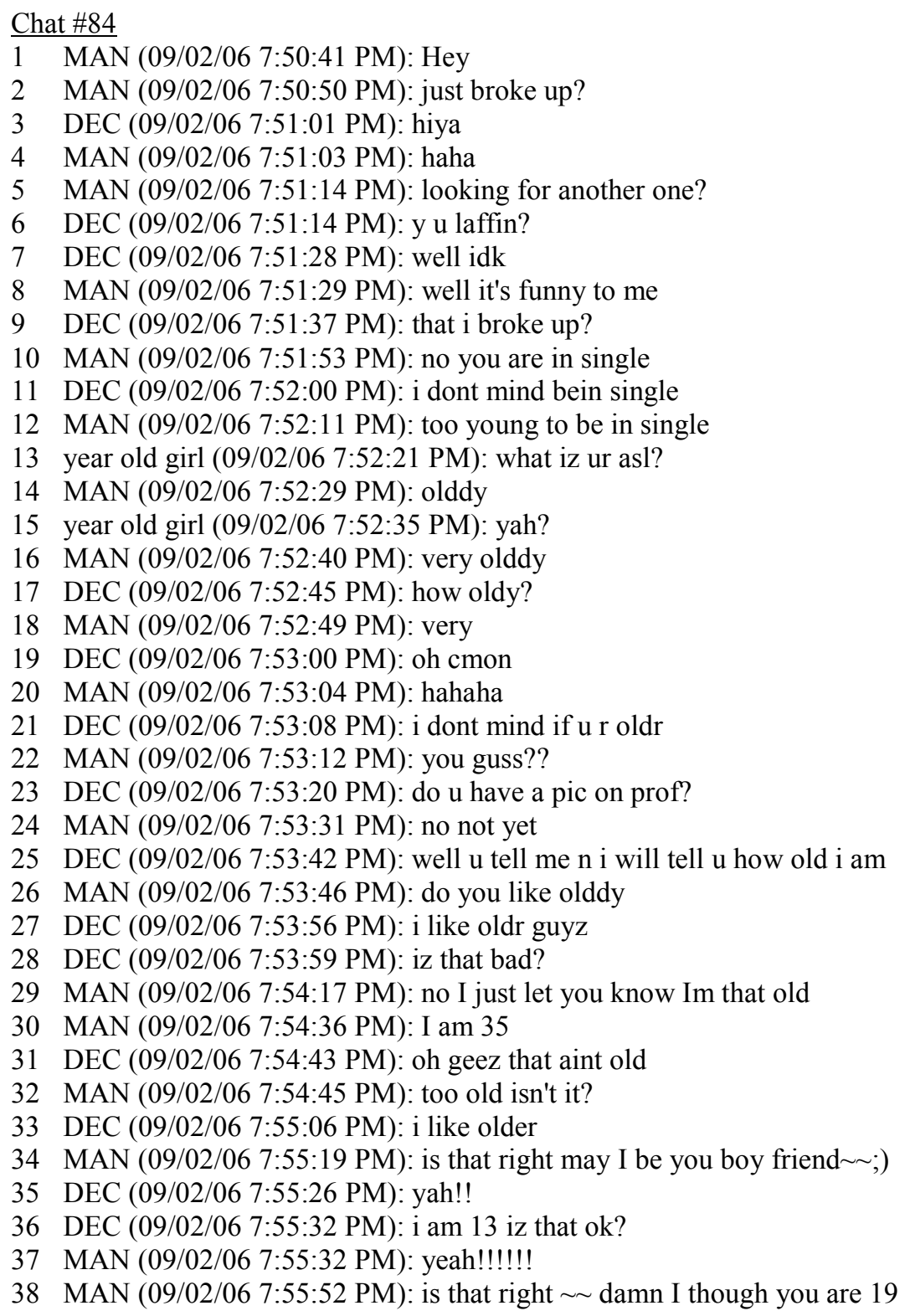

\section{Chat \#85}

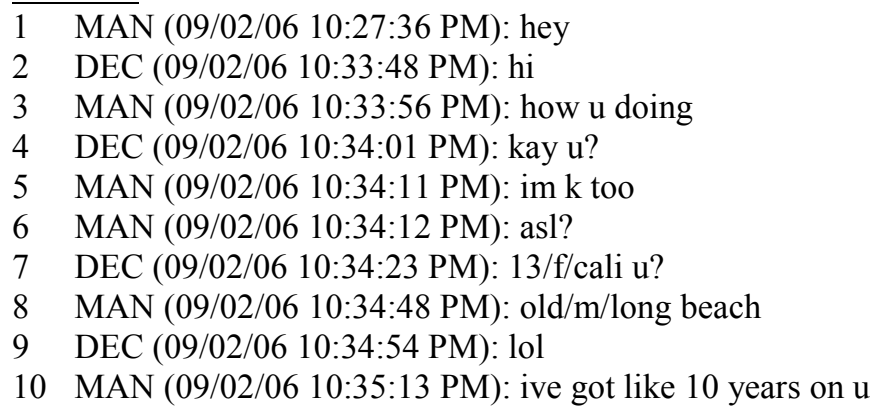


11 DEC (09/02/06 10:35:20 PM): ur 23?

12 MAN (09/02/06 10:35:29 PM): yes young lady i am

13 DEC (09/02/06 10:35:33 PM): lol

14 DEC (09/02/06 10:35:37 PM): thats notold

15 DEC (09/02/06 10:35:44 PM): like 80 is old

16 MAN (09/02/06 10:35:50 PM): well no its not, but to u its old

17 DEC (09/02/06 10:36:22 PM): i dont think ur old lol

18 MAN (09/02/06 10:36:42 PM): ok, too old for u to date and stuff

19 DEC (09/02/06 10:37:01 PM): ya ok watevr lol

20 MAN (09/02/06 10:38:27 PM): so where do u live young lady

21 DEC (09/02/06 10:38:43 PM): near LA

22 MAN (09/02/06 10:39:01 PM): what city

23 DEC (09/02/06 10:39:09 PM): its a secret lol

24 MAN (09/02/06 10:39:29 PM): lol not its not

25 DEC (09/02/06 10:39:36 PM): ya it is

26 DEC (09/02/06 10:39:40 PM): i dont no u

27 MAN (09/02/06 10:40:39 PM): oh ok

28 DEC (09/02/06 10:40:49 PM): :)

29 MAN (09/02/06 10:40:53 PM): u look so young

30 DEC (09/02/06 10:41:13 PM): :(

31 MAN (09/02/06 10:41:23 PM): well u are 13

32 DEC (09/02/06 10:41:29 PM): im not a little kid

33 MAN (09/02/06 10:41:34 PM): sure u are

34 MAN (09/02/06 10:41:40 PM): ull be perfect in 5 years lol

35 DEC (09/02/06 10:41:51 PM): no im not

36 MAN (09/02/06 10:42:14 PM): ur not what

37 DEC (09/02/06 10:42:26 PM): a little kid

38 MAN (09/02/06 10:43:06 PM): if u say so

39 MAN (09/02/06 10:49:00 PM): so what u doing 13_year_old_girl

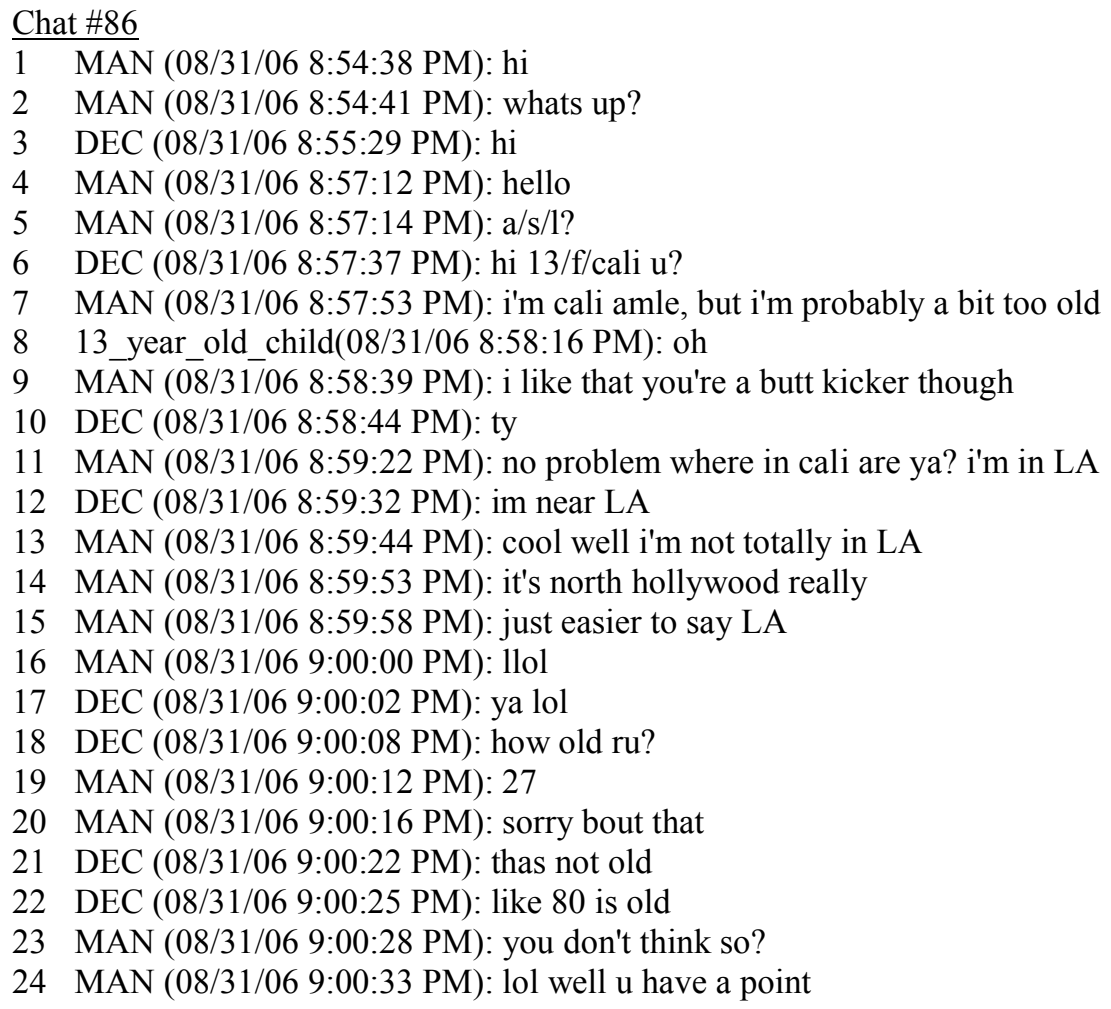


$25 \operatorname{DEC}(08 / 31 / 06$ 9:00:34 PM): noooo

26 MAN (08/31/06 9:00:39 PM): well older than u i guess

27 DEC (08/31/06 9:00:45 PM): well ya lol

28 MAN (08/31/06 9:01:08 PM): sorry i was expecting you to be like in your 20's and then i was going to flirt lol

29 DEC (08/31/06 9:01:23 PM): relly u think i look that old?

30 MAN (08/31/06 9:01:34 PM): no, before i saw you're pic

31 DEC (08/31/06 9:01:38 PM): oh

32 DEC (08/31/06 9:01:39 PM): :(

33 MAN (08/31/06 9:01:56 PM): why are you sad?

34 DEC (08/31/06 9:02:05 PM): i thot i look older mebbe

35 MAN (08/31/06 9:02:30 PM): well you look a little older, but you're really cute, so does that help?

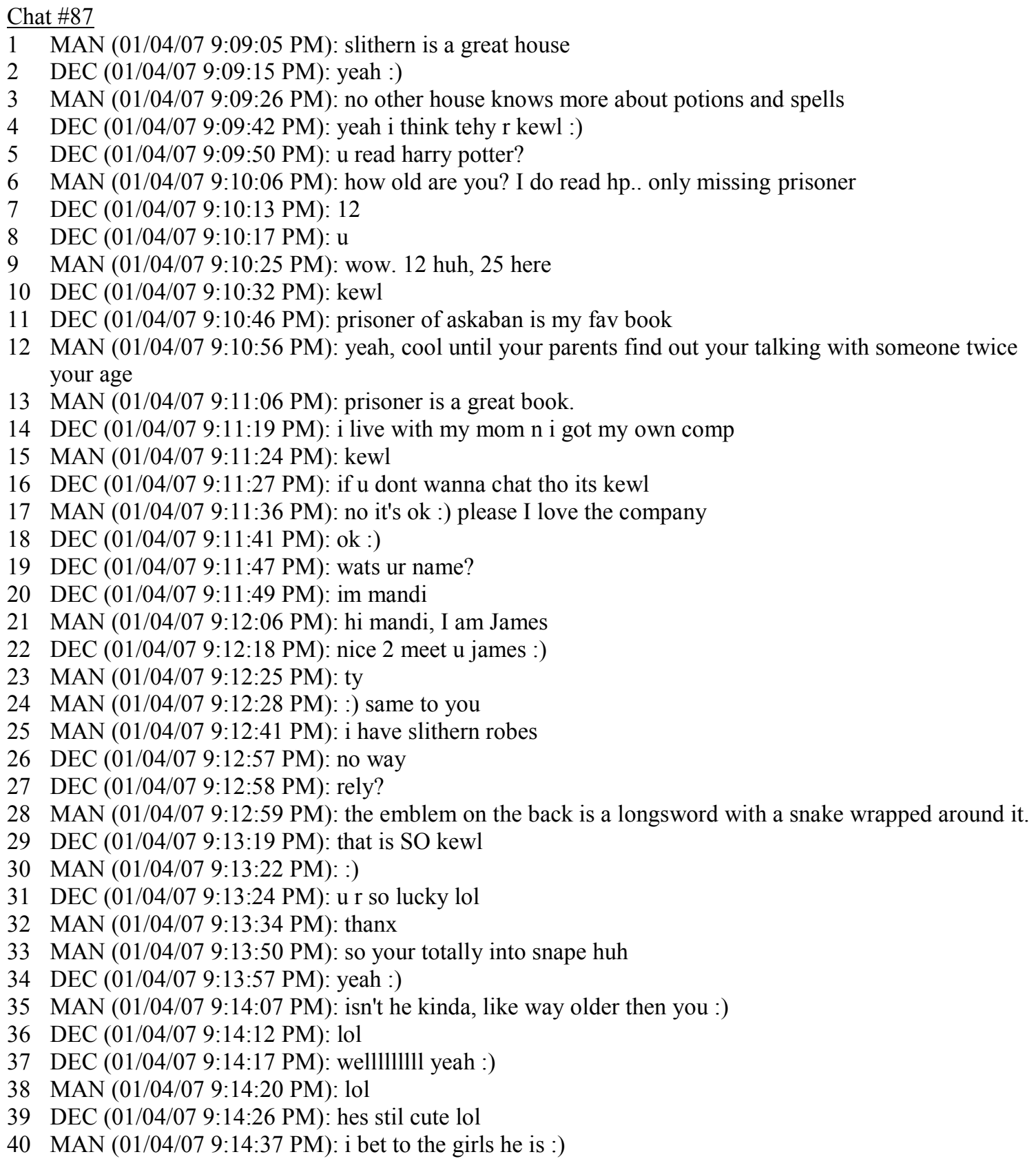


$41 \operatorname{DEC}(01 / 04 / 07$ 9:14:42 PM): lol

$42 \operatorname{MAN}(01 / 04 / 07$ 9:14:46 PM): has a great sense of humor too

Chat \#88

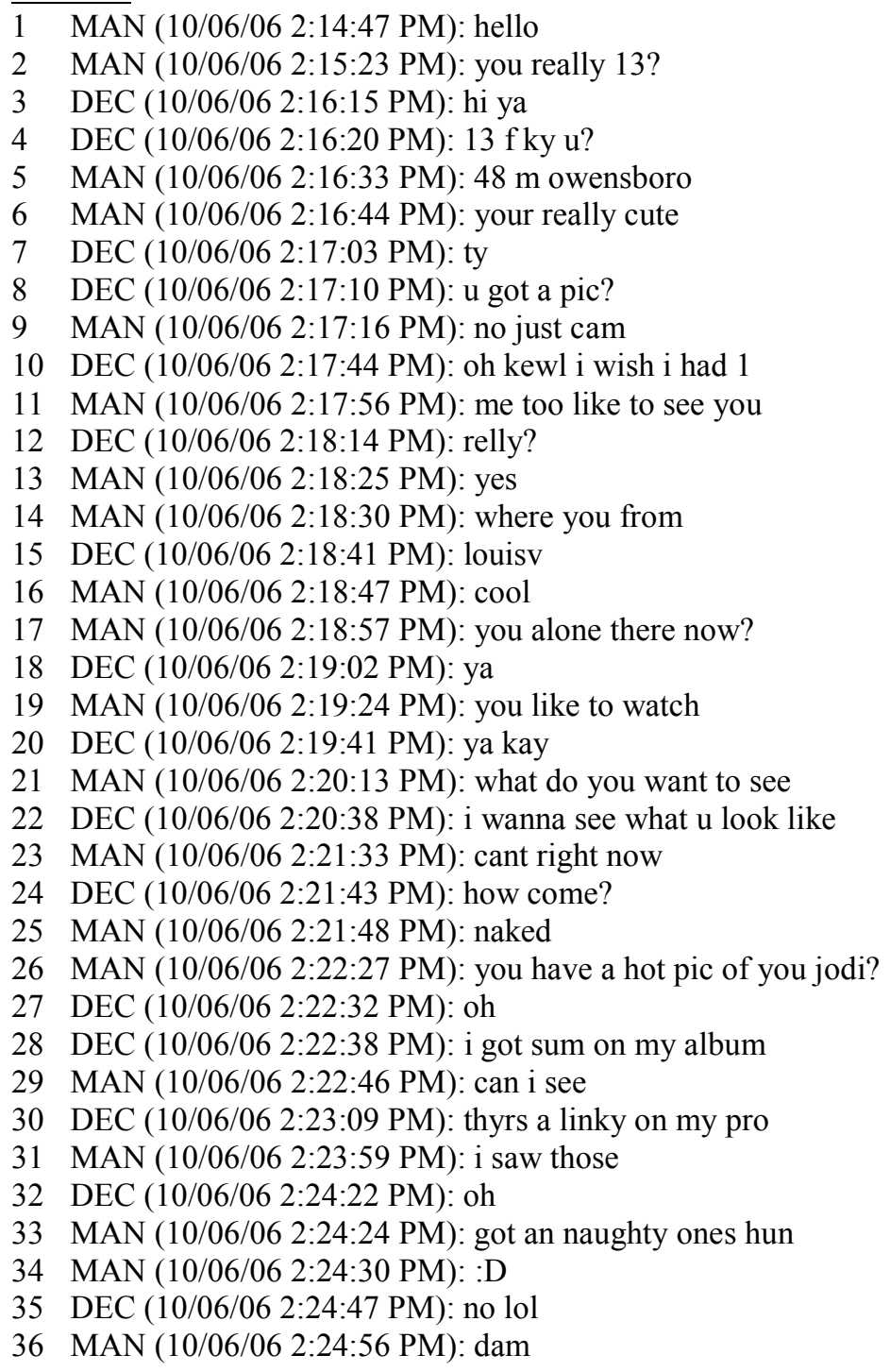

Chat \#89

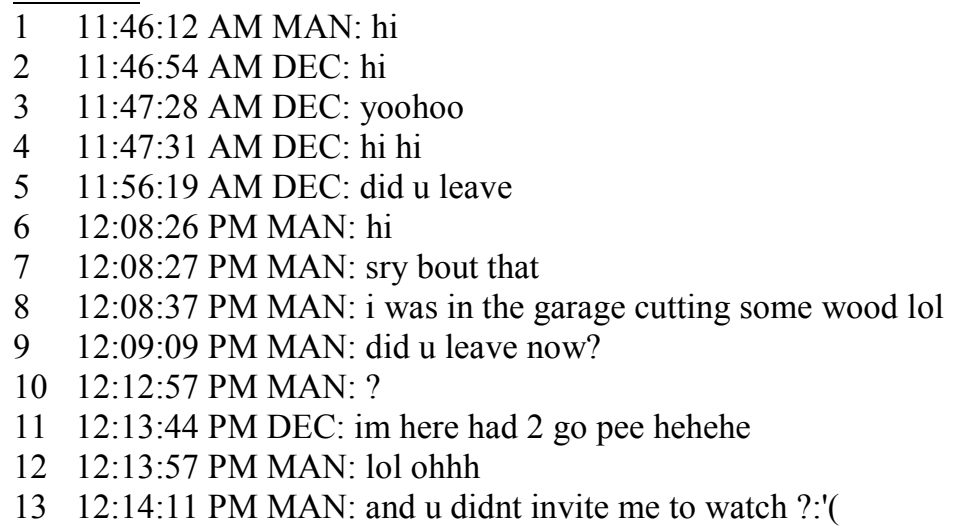


14 12:14:17 PM DEC: hehehe

15 12:14:35 PM DEC: kk so come watch

16 12:14:41 PM MAN: where ustay

17 12:14:52 PM DEC: live in fort myers

18 12:14:56 PM DEC: a/s/l

19 12:15:00 PM MAN: ohh im in ft lauderdale

20 12:15:05 PM MAN: i may $b$ a bit old for $u$ tho lol

21 12:15:17 PM MAN: i'll b 21 in july

22 12:15:18 PM DEC: wat u like 90 and wrinkled

23 12:15:25 PM DEC: hehehe

24 12:15:37 PM DEC: my old bf wuz 24

25 12:15:43 PM MAN: ohh

26 12:16:03 PM DEC: im cindy

27 12:16:05 PM MAN: im marvin

28 12:16:07 PM MAN: pixx?

29 12:16:16 PM DEC: u got a pic

30 12:16:19 PM MAN: send ures

31 12:16:25 PM DEC: u gonna send one

32 12:16:38 PM MAN: ima send more than 1

33 12:16:40 PM MAN: $u$ do da same

34 12:17:11 PM DEC: kk i gotta email cauz i dont no how to do the other so u gotta email pix 2 me

35 12:17:17 PM MAN: $\mathrm{k}$

36 12:17:18 PM DEC: brb and ill send

37 12:17:43 PM MAN: u wanna trade $\mathrm{x}$ rated ones too or no

\section{Chat $\# 90$}

1 MAN (2:23:02 AM): hello

2 DEC (2:23:53 AM): hi asl?

3 MAN (2:24:09 AM): 32/m/norwalk/562 and you

4 DEC (2:24:24 AM): 13 f laguna

5 MAN (2:25:12 AM): for reals 13?

6 DEC (2:25:19 AM): lol yah

7 MAN (2:25:38 AM): lol....ok, you don't mind chatting?

8 DEC (2:25:47 AM): naw im kewl u?

9 MAN (2:26:14 AM): ok

10 MAN (2:26:26 AM): so what you doing still up

11 DEC (2:27:11 AM): jus being bored $u$ ?

12 MAN (2:27:38 AM): same here

13 MAN (2:27:53 AM): bored and nothing good on tv

14 DEC (2:28:16 AM): lol yah

15 MAN (2:29:04 AM): lol...I usually don't talk with anyone your age but to tell the truth it's hard enough to find anyone real in this chat rooms now. They all seem to be sex bots now

16 DEC (2:29:22 AM): lol yah i know!! its all spam

17 MAN (2:29:28 AM): yup

18 MAN (2:29:40 AM): so whatcha wanna talk about

19 DEC (2:30:00 AM): whatev u want

20 MAN (2:30:41 AM): lol.... think I'll let you decide were are conversation goes....lol....I'd feel better if that is ok

21 MAN (2:31:22 AM): is that ok

22 MAN (2:32:18 AM): are you still there?

23 DEC (2:33:08 AM): yah im here

24 DEC (2:33:18 AM): do u go 2 school?

25 MAN (2:33:40 AM): no I work as a dockworker

26 MAN (2:33:57 AM): you can see my picture right?

27 DEC (2:34:59 AM): yup 
28 DEC (2:35:02 AM): ur realy cute

29 MAN (2:35:14 AM): lol....thanks

30 MAN (2:36:20 AM): so you must have a boyfriend?

31 DEC (2:36:53 AM): lol no

32 MAN (2:37:02 AM): why not

33 DEC (2:37:55 AM): tha 1 guy i like is datin this totaly slutty girl

34 DEC (2:38:02 AM): tha rest of tha guyz i know $r$ dumb

35 MAN (2:38:05 AM): lol bummer

\section{Chat \#91}

1 First line was something like "Hey"

2 DEC (10:40:21 PM): hey

3 MAN (10:40:39 PM): how re u?

4 DEC (10:40:55 PM): alright, bored

5 DEC (10:40:55 PM): lol

6 MAN (10:41:01 PM): same here

7 MAN (10:41:04 PM): sux

8 MAN (10:41:48 PM): what $r$ u up to?

9 DEC (10:41:52 PM): nuthin

10 DEC (10:41:57 PM): so bored im crazy

11 MAN (10:42:00 PM): lol

12 MAN (10:42:02 PM): ur crazy

13 MAN (10:42:09 PM): ?

14 MAN (10:42:13 PM): ur really cute lol

15 MAN (10:42:17 PM): but u look really young

16 MAN (10:42:20 PM): how old $r$ u?

17 DEC (10:42:24 PM): um

18 DEC (10:42:26 PM): 13

19 DEC (10:42:27 PM): lol

20 quin says (10:42:32 PM): damn

21 MAN (10:42:33 PM): lol

22 DEC (10:42:42 PM): sorry

23 MAN (10:42:48 PM): its ok

24 MAN (10:54:36 PM): so how was ur day?

25 DEC (10:54:43 PM): boring

26 DEC (10:54:45 PM): you?

27 MAN (10:54:50 PM): lol

28 MAN (10:54:54 PM): same i guess

29 MAN (10:54:56 PM): what did u do

30 DEC (10:55:15 PM): sat around bein bored

31 DEC (10:55:17 PM): not in school yet

32 MAN (10:55:22 PM): lol

33 MAN (10:55:25 PM): when do u sart school

\section{Chat \#92}

1 MAN hey, you're a cutie

2 DEC (10:55:59 PM): ha thanks

3 MAN (10:56:07 PM): lol, ya bet

4 DEC (10:56:46 PM): asl f 14 here

5 MAN (10:57:04 PM): oh my

6 MAN (10:57:05 PM): 34

7 MAN (10:57:09 PM): lol, sorry girl

8 DEC (10:57:21 PM): what 4

9 MAN (10:57:22 PM): don't mean to offend you

10 MAN (10:57:33 PM): you gotta cam? 
11 DEC (10:58:26 PM): no mom wont let me have 1

12 MAN (10:58:35 PM): prob smart

13 DEC (10:59:27 PM): so what $r \mathrm{u}$ doin 2nite

14 MAN (10:59:57 PM): nuttin, trying to hook up

15 DEC (11:00:51 PM): so $u$ tht i was cute wow no one ever told me that b4

16 MAN (11:01:07 PM): lol... duh

17 MAN (11:01:09 PM): lol

18 MAN (11:01:11 PM): sorry

19 DEC (11:01:54 PM): so u dont want to talk with me then

20 MAN (11:02:09 PM): nah, its cool... just feel weird

21 DEC (11:02:25 PM): oh i dont feel werid

22 MAN (11:02:35 PM): i do... it's diff for me

23 DEC (11:02:52 PM): y

24 MAN (11:03:06 PM): i dunno

25 MAN (11:03:17 PM): if you don't have a cam, or get naked, i guess its okay

26 DEC (11:03:32 PM): iget naked when i get a shower ha

27 MAN (11:03:43 PM): lol, good idea

28 DEC (11:03:44 PM): but no cam

29 MAN (11:03:48 PM): i bet you look good too

30 DEC (11:03:57 PM): dunno

31 DEC (11:04:09 PM): oh ur cute 2 btw

32 MAN (11:04:17 PM): lol... thanks

33 MAN (11:04:27 PM): don't flirt, it'll get me in trouble

\section{Chat \#93}

1 MAN (1:43:08 PM): hey

2 MAN (1:43:09 PM): hows it going?

3 DEC (1:43:49 PM): ok

4 DEC (1:43:53 PM): how ru

5 MAN (1:43:55 PM): purty good thx

6 MAN (1:43:58 PM): asl?

7 DEC (1:43:58 PM): asl

8 MAN (1:44:02 PM): lol

9 MAN (1:44:05 PM): 30/m/Findlay

10 MAN (1:44:10 PM): u?

11 DEC (1:44:16 PM): 13/f/OH

12 MAN (1:44:33 PM): bummer :((

13 DEC (1:44:43 PM): lol

14 DEC (1:44:58 PM): wheres Findlay

15 MAN (1:45:06 PM): NW Ohio

16 MAN (1:45:11 PM): where $u$ at?

17 DEC (1:45:20 PM): outside of Dayton

18 MAN (1:45:46 PM): yeah I'm about 1.5 hours north of dayton on $i 75$

19 DEC (1:45:54 PM): kewl

20 MAN (1:46:01 PM): yup yup

21 DEC (1:46:03 PM): so what $u$ doin

22 MAN (1:46:09 PM): looking for some fun tonght

23 MAN (1:46:10 PM): u?

24 DEC (1:46:15 PM): lol

25 DEC (1:46:29 PM): my moms in Vegas til tomorrw

26 MAN (1:46:39 PM): WOHOO!

27 DEC (1:46:42 PM): lol

28 DEC (1:46:48 PM): yah but i dont drive

$29 \operatorname{MAN}(1: 46: 53 \mathrm{PM})$ : not at 13 ;)

30 MAN (1:47:03 PM): so u alone then? 
31 DEC (1:47:54 PM): yup

32 MAN (1:47:59 PM): bummer

33 DEC (1:48:09 PM): i babysit other peeps kids so why not babysit me lol

$34 \operatorname{MAN}(1: 48: 18 \mathrm{PM})$ : who me babysit you?

35 DEC (1:48:34 PM): i know we got a way big house....ooooo...scary lol

$36 \operatorname{MAN}(1: 48: 41 \mathrm{PM}): ;))$

Chat \#94

1 MAN (09/24/08 10:13:40 PM): Hello carriebare and what are you up to?

2 DEC (09/24/08 10:14:23 PM): hi

3 MAN (09/24/08 10:14:40 PM): hi

4 MAN (09/24/08 10:15:06 PM): what are you looking for tonight

5 DEC (09/24/08 10:15:13 PM): just chattin

6 MAN (09/24/08 10:15:29 PM): cool and are you busy

7 DEC (09/24/08 10:15:38 PM): nah i was away from the puter

8 MAN (09/24/08 10:15:58 PM): so what would you like to chat about

9 DEC (09/24/08 10:16:11 PM): wat is ur asl?

10 MAN (09/24/08 10:16:35 PM): lol old enought 57/m/indiana

11 DEC (09/24/08 10:16:43 PM): lol im $14 \mathrm{f} \mathrm{mi}$

12 MAN (09/24/08 10:17:06 PM): cool

13 DEC (09/24/08 10:17:16 PM): i just looked at ur prof u r cute

$14 \operatorname{MAN}(09 / 24 / 08$ 10:17:24 PM): well ty

15 DEC (09/24/08 10:17:35 PM): lol im nosey

16 MAN (09/24/08 10:18:01 PM): ok and what do you like to do for excitement

17 DEC (09/24/08 10:18:23 PM): well i like to skateboard music movies hanging with friends chatting

18 DEC (09/24/08 10:18:24 PM): u?

19 MAN (09/24/08 10:18:49 PM): oh camping rideing horses and sex

$20 \operatorname{DEC}(09 / 24 / 08$ 10:18:57 PM): lol

21 DEC (09/24/08 10:19:02 PM): thats kool

22 MAN (09/24/08 10:19:34 PM): glad you like and do you camp any

23 DEC (09/24/08 10:19:43 PM): no i never been camping before

24 MAN (09/24/08 10:20:14 PM): oh you are missing a good time and some great food fixed over the fire

25 DEC (09/24/08 10:20:33 PM): well noone ever took me before they ; $r$ all too busy i guess

26 MAN (09/24/08 10:21:30 PM): that is to bad i work a 47.5 hours aweek at my job and farm and still find time to go camping

27 DEC (09/24/08 10:21:39 PM): wow

28 DEC (09/24/08 10:21:51 PM): well i guess thats just how it goes

29 MAN (09/24/08 10:22:00 PM): yep

30 MAN (09/24/08 10:22:11 PM): so have you ever rode a horse

31 DEC (09/24/08 10:22:15 PM): no

32 MAN (09/24/08 10:22:30 PM): fishing

33 DEC (09/24/08 10:22:48 PM): well my dad took me once when $\mathrm{i}$ was 10

34 MAN (09/24/08 10:22:58 PM): oh

35 DEC (09/24/08 10:23:08 PM): it was fun

36 MAN (09/24/08 10:23:17 PM): yes it is

37 DEC (09/24/08 10:23:29 PM): u do it lots?

38 MAN (09/24/08 10:23:38 PM): so have you tried sex yet

39 MAN (09/24/08 10:23:45 PM): every chance i get

Chat \#95

1 MAN (2:10:59 PM): hello Natalie....how are you doing today?

2 DEC (2:12:01 PM): good u?

3 DEC (2:12:02 PM): asl?

$4 \operatorname{MAN}(2: 12: 19 \mathrm{PM}): 27 / \mathrm{m} /$ Atlanta

5 MAN (2:12:20 PM): you 
6 DEC (2:12:35 PM): $15 \mathrm{fga}$

7 MAN (2:12:41 PM): oh

8 MAN (2:13:19 PM): hows your day

9 DEC (2:13:30 PM): boring lol

10 MAN (2:13:36 PM): i'm sorry to hear that

11 MAN (2:14:38 PM): what part of town are you in

12 DEC (2:18:38 PM): brb sorry

13 MAN (2:18:47 PM): ok

14 DEC (2:19:32 PM): im near columbus

15 MAN (2:19:41 PM): i see

16 MAN (2:19:47 PM): have you been to Atlanta

17 DEC (2:20:03 PM): no not yete

18 DEC (2:20:05 PM): yet

19 DEC (2:20:08 PM): i just moved here

20 MAN (2:20:12 PM): oh realy

21 MAN (2:20:16 PM): from were

22 DEC (2:20:34 PM): st louis

23 MAN (2:20:52 PM): i used to live in Hazlewood Mo

24 MAN (2:20:58 PM): near Florissant

25 DEC (2:21:14 PM): omg

26 DEC (2:21:16 PM): really?

27 DEC (2:21:18 PM): ;D

28 MAN (2:21:19 PM): yeah

29 DEC (2:21:19 PM): :D

30 DEC (2:21:22 PM): kewl

31 DEC (2:21:29 PM): $\mathrm{i}$ know where that is

32 MAN (2:21:44 PM): how long you been hear

33 DEC (2:21:52 PM): about a month

34 MAN (2:21:57 PM): realy

35 MAN (2:22:03 PM): you moved with parents

36 DEC (2:22:06 PM): yeah

37 DEC (2:22:17 PM): we used 2 live in kirkwood

38 MAN (2:22:24 PM): i know were that is

39 DEC (2:25:30 PM): lol

40 DEC (2:25:47 PM): how long have u been here

41 MAN (2:25:49 PM): are you busy

\section{Chat \#96}

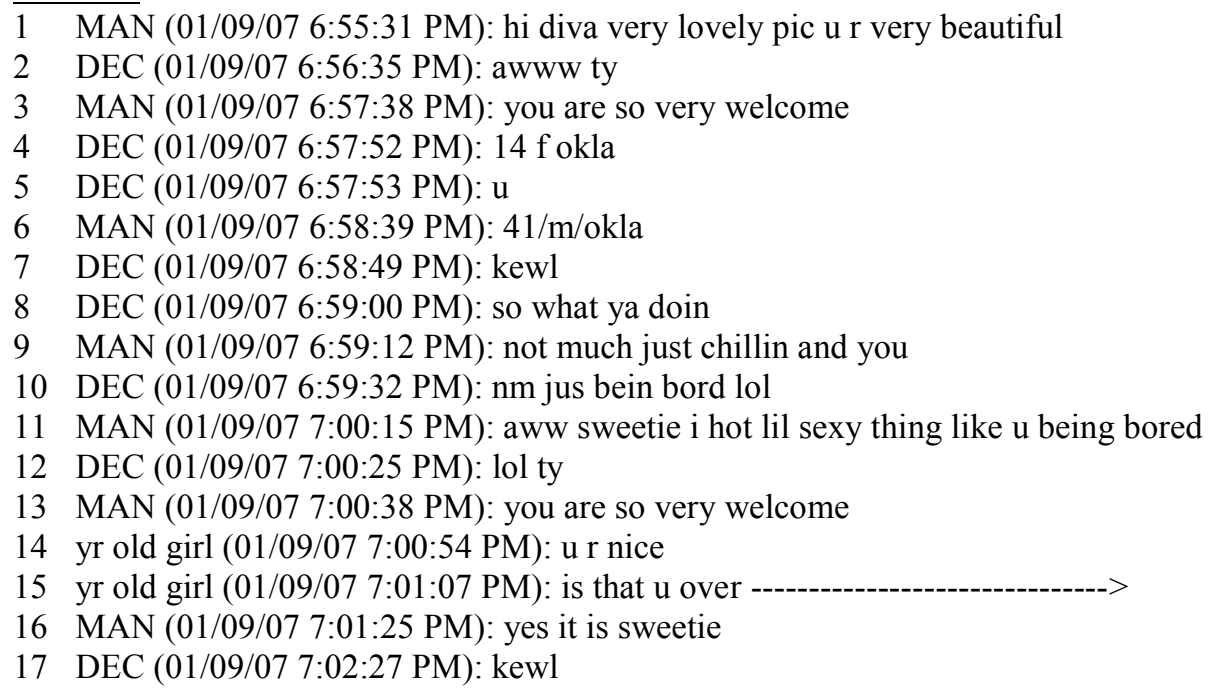


$18 \operatorname{MAN}(01 / 09 / 07$ 7:02:38 PM): ty u sweetie

$19 \operatorname{MAN}(01 / 09 / 07$ 7:03:46 PM): what do u like to do for fun

20 DEC (01/09/07 7:04:04 PM): shop hang wit frends campin

21 DEC (01/09/07 7:04:09 PM): jus normal stuff

22 DEC (01/09/07 7:04:11 PM): lol

23 DEC (01/09/07 7:04:13 PM): u

24 MAN (01/09/07 7:05:12 PM): camping fishing hanging out with friends parting dancing

25 DEC (01/09/07 7:05:24 PM): kewl

26 DEC (01/09/07 7:05:34 PM): i dont lik fishin ewwwwwwww lol

27 MAN (01/09/07 7:06:00 PM): well thats ok sweetie

28 DEC (01/09/07 7:06:08 PM): lol kewl

$29 \operatorname{MAN}(01 / 09 / 07$ 7:06:35 PM): yeah sweetie

30 MAN (01/09/07 7:07:21 PM): i also like movies and cuddlin too

31 DEC (01/09/07 7:07:29 PM): thats kewl too

$32 \operatorname{MAN}(01 / 09 / 07$ 7:07:44 PM): yeah it is too

33 DEC (01/09/07 7:09:25 PM): ur quiet lol

34 MAN (01/09/07 7:09:43 PM): sorry sweetie

35 DEC (01/09/07 7:09:50 PM): its k

36 MAN (01/09/07 7:10:04 PM): so do u like older guys

37 DEC (01/09/07 7:10:16 PM): i gues so its jus a numbr to me

38 DEC (01/09/07 7:10:29 PM): i lik chattin to whoevr

$39 \operatorname{MAN}(01 / 09 / 07$ 7:10:39 PM): cool and yea me too

40 DEC (01/09/07 7:10:44 PM): kewl

41 DEC (01/09/07 7:11:00 PM): so u lik kiss groop huh

42 MAN (01/09/07 7:11:17 PM): yes i do they rock

43 DEC (01/09/07 7:11:28 PM): lol ya

44 MAN (01/09/07 7:12:28 PM): ohh ya what kind of music do u like

$45 \operatorname{DEC}(01 / 09 / 07$ 7:12:43 PM): contry mostly

$46 \operatorname{MAN}(01 / 09 / 07$ 7:13:01 PM): cool

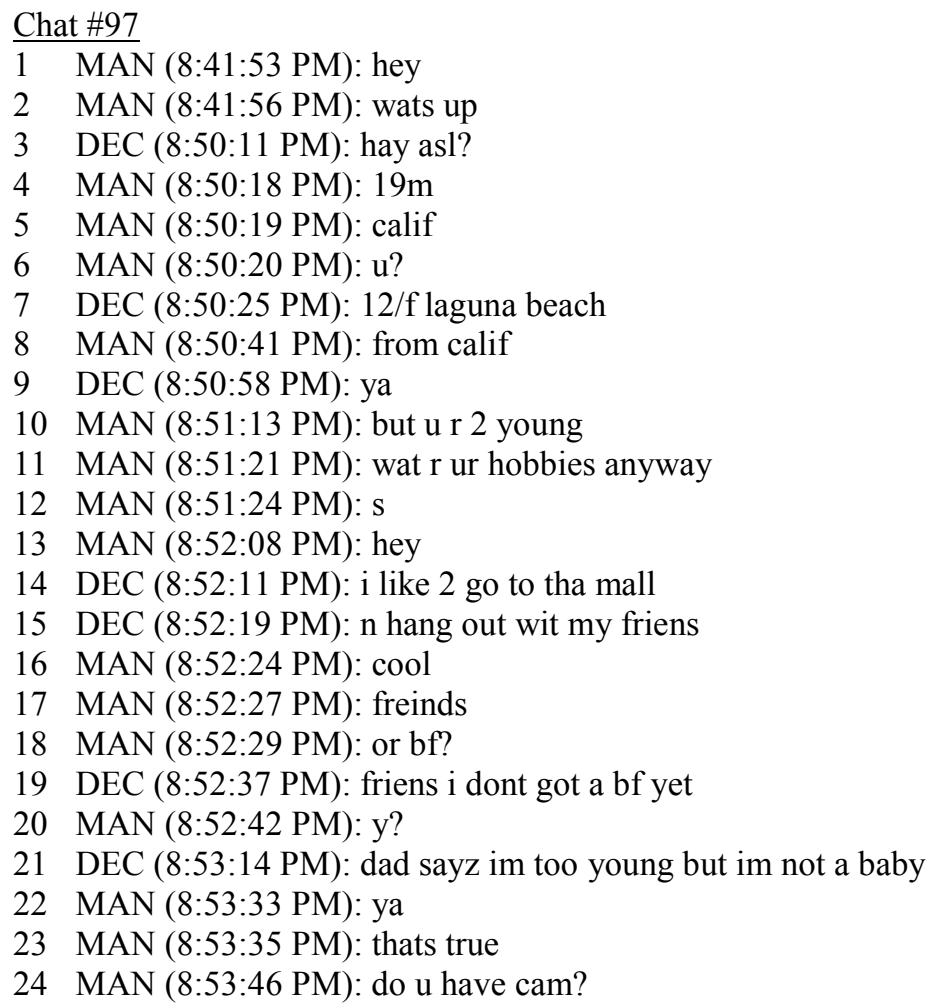


25 DEC (8:54:35 PM): no dad took it away when $i$ threw it at him cuz i got mad

26 MAN (8:55:11 PM): lol

27 MAN (8:55:17 PM): u did u get mad

28 MAN (8:55:37 PM): $y^{*}$

\section{Chat \#98}

1 MAN (07/18/06 9:40:01 PM): pls excuse my intrusion,hi i'm joe a single white male/41 and i'd like to say $i$ think your an attractive woman indeed,as well as sexy...i'll not intrude again tho.

2 DEC (07/18/06 9:40:10 PM): wow thanks

3 MAN (07/18/06 9:40:47 PM): lol your welcome,dont be shocked ,hell i'm sure most men think the same

4 DEC (07/18/06 9:41:10 PM): i dunno most think im black so that sbad or whatever

5 DEC (07/18/06 9:41:13 PM): whats yoru asl?

6 MAN (07/18/06 9:42:29 PM): i'm 41/southside of atl in fayetteville/man...and if they think that because your black then their just ignorant and were'nt raised right

7 MAN (07/18/06 9:43:05 PM): i'm 5'11/170lbs/natural tan/hazel eye's/haiy chest\&stomach

8 DEC (07/18/06 9:43:16 PM): yeah a lotta people werent raised right 15/f/no of columbus

$9 \operatorname{MAN}(07 / 18 / 06$ 9:43:42 PM): well we wont talk about them lol

10 DEC (07/18/06 9:43:50 PM): lol good :)

11 MAN (07/18/06 9:44:14 PM): i'm maby an hour from columbus,not far at all

12 DEC (07/18/06 9:44:20 PM): wow thats cool

13 MAN (07/18/06 9:44:36 PM): so can i ask,am i to old for you to talk to and all?

14 DEC (07/18/06 9:44:45 PM): lol no your not a grandpa

15 MAN (07/18/06 9:44:54 PM): i know thats right lol

16 MAN (07/18/06 9:45:19 PM): i'm damn healthy and active,keep a young mind and spirit then you'll be fine

17 DEC (07/18/06 9:45:20 PM): lol cool then

18 DEC (07/18/06 9:45:29 PM): yeah age is just a numbah thats what allaiyah sang

19 DEC (07/18/06 9:45:40 PM): its just like color aint mean nothing

20 MAN (07/18/06 9:46:04 PM): your right,so were good then

21 DEC (07/18/06 9:46:15 PM): yeah all good to me

22 MAN (07/18/06 9:46:22 PM): so what are your interest ?

23 DEC (07/18/06 9:46:30 PM): what do you mean?

24 MAN (07/18/06 9:46:37 PM): well as long as it's good to $u$ thats all that matters i'd say

25 DEC (07/18/06 9:46:45 PM): yeah

26 DEC (07/18/06 9:47:54 PM): whats your name you gotta pic?

27 MAN (07/18/06 9:48:16 PM): as in what do you like to do for fun,it dont matter if it's just goin out or even if it's of the adult nature, i'm not shy...me i dont like clubs much, to much noise, i kinda like the one on one walks and talks.you can learn alot that way

28 DEC (07/18/06 9:48:48 PM): i cant go to clubs i aint old enough to and theres not much to do here i liked going to jamaca

29 MAN (07/18/06 9:49:33 PM): yeah your in a bad place for fun, no doubt..but hey there's fun to be had at the right times ..

30 MAN (07/18/06 9:49:45 PM): so i take it you live at home?

31 DEC (07/18/06 9:49:51 PM): yeah i live with my mom

32 MAN (07/18/06 9:50:53 PM): it's cool $i$ asked only because if it were to happen and we were gonna hook up i dont want you to get in trouble and well i just say what we say and talk about is our buisness

33 MAN (07/18/06 9:51:09 PM): i'm not sayin we are now ,but just if lol

34 DEC (07/18/06 9:51:22 PM): yeah i know i wouldnt wanna get in trouble either i know whats what

35 MAN (07/18/06 9:51:57 PM): i'm sure tho it could be worked out if it happened

36 MAN (07/18/06 9:52:18 PM): but i honestly do think ur attractive and damn sexy

37 DEC (07/18/06 9:52:30 PM): thanks thats nice to hear for a change

38 DEC (07/18/06 9:52:33 PM): i like hearing that

39 MAN (07/18/06 9:52:51 PM): well it's only true 
Chat \#99

\begin{tabular}{ll}
\hline 1 & MAN $(03 / 08 / 08$ 6:56:02 PM): hi \\
2 & DEC (03/08/08 6:56:36 PM): hi \\
3 & MAN (03/08/08 6:57:02 PM): how you doin \\
4 & MAN (03/08/08 6:57:53 PM): ? \\
5 & DEC (03/08/08 6:58:16 PM): i am k u? \\
6 & MAN (03/08/08 6:58:34 PM): iam good \\
7 & MAN (03/08/08 6:59:17 PM): asl? \\
8 & DEC (03/08/08 6:59:22 PM): 14 f \\
9 & DEC (03/08/08 6:59:23 PM): u? \\
10 & MAN (03/08/08 6:59:42 PM): 21 m \\
11 & DEC (03/08/08 7:00:08 PM): cool \\
12 & MAN (03/08/08 7:00:42 PM): how is your day going? \\
13 & DEC (03/08/08 7:00:52 PM): freekin boring \\
14 & MAN (03/08/08 7:01:07 PM): i know what you mean \\
15 & MAN (03/08/08 7:01:53 PM): iam bout to leave here soon \\
16 & DEC (03/08/08 7:02:05 PM): where u going \\
17 & MAN (03/08/08 7:02:11 PM): to my house \\
18 & DEC (03/08/08 7:02:17 PM): where are u now \\
19 & MAN (03/08/08 7:02:24 PM): my parents \\
20 & DEC (03/08/08 7:02:28 PM): kk \\
21 & DEC (03/08/08 7:02:31 PM): where that \\
22 & MAN (03/08/08 7:02:46 PM): my parents or my house? \\
23 & MAN (03/08/08 7:03:31 PM): i live in hazel park \\
24 & MAN (03/08/08 7:03:44 PM): my parents live in southfield \\
25 & MAN (03/08/08 7:04:32 PM): so iam going back to hazel park \\
26 & MAN (03/08/08 7:04:48 PM): iam just visiting here \\
27 & DEC (03/08/08 7:05:06 PM): i live in beeleville \\
28 & DEC (03/08/08 7:05:13 PM): belleville \\
29 & MAN (03/08/08 7:05:21 PM): yeah \\
30 & MAN (03/08/08 7:06:04 PM): ive been there \\
31 & MAN (03/08/08 7:07:53 PM): its very diverse there \\
32 & DEC (03/08/08 7:08:01 PM): yah i guss so \\
33 & MAN (03/08/08 7:08:20 PM): so what are you doing on here?
\end{tabular}

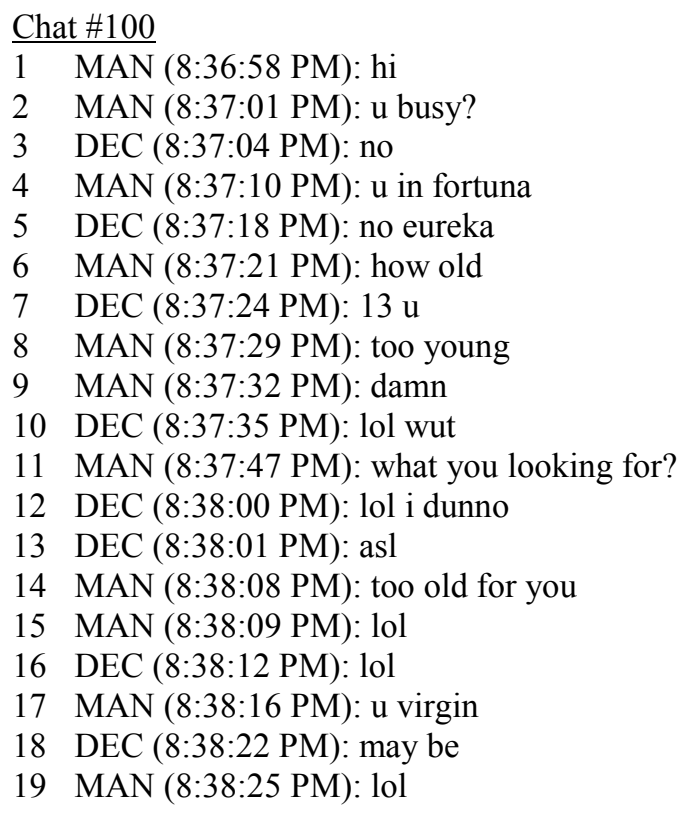


20 MAN (8:38:27 PM): you are

21 MAN (8:38:29 PM): lol

22 DEC (8:38:31 PM): may be

23 MAN (8:38:44 PM): u lookin to get it off ya

24 DEC (8:38:53 PM): may be

25 MAN (8:39:01 PM): let'

26 MAN (8:39:09 PM): let's do it

27 DEC (8:39:15 PM): lol asl

28 MAN (8:39:25 PM): u got cam or more pics

29 DEC (8:39:32 PM): nah

30 MAN (8:39:38 PM): oh well

31 MAN (8:39:39 PM): cya

32 DEC (8:39:52 PM): wutever by than

33 MAN (8:39:58 PM): bye cute 